
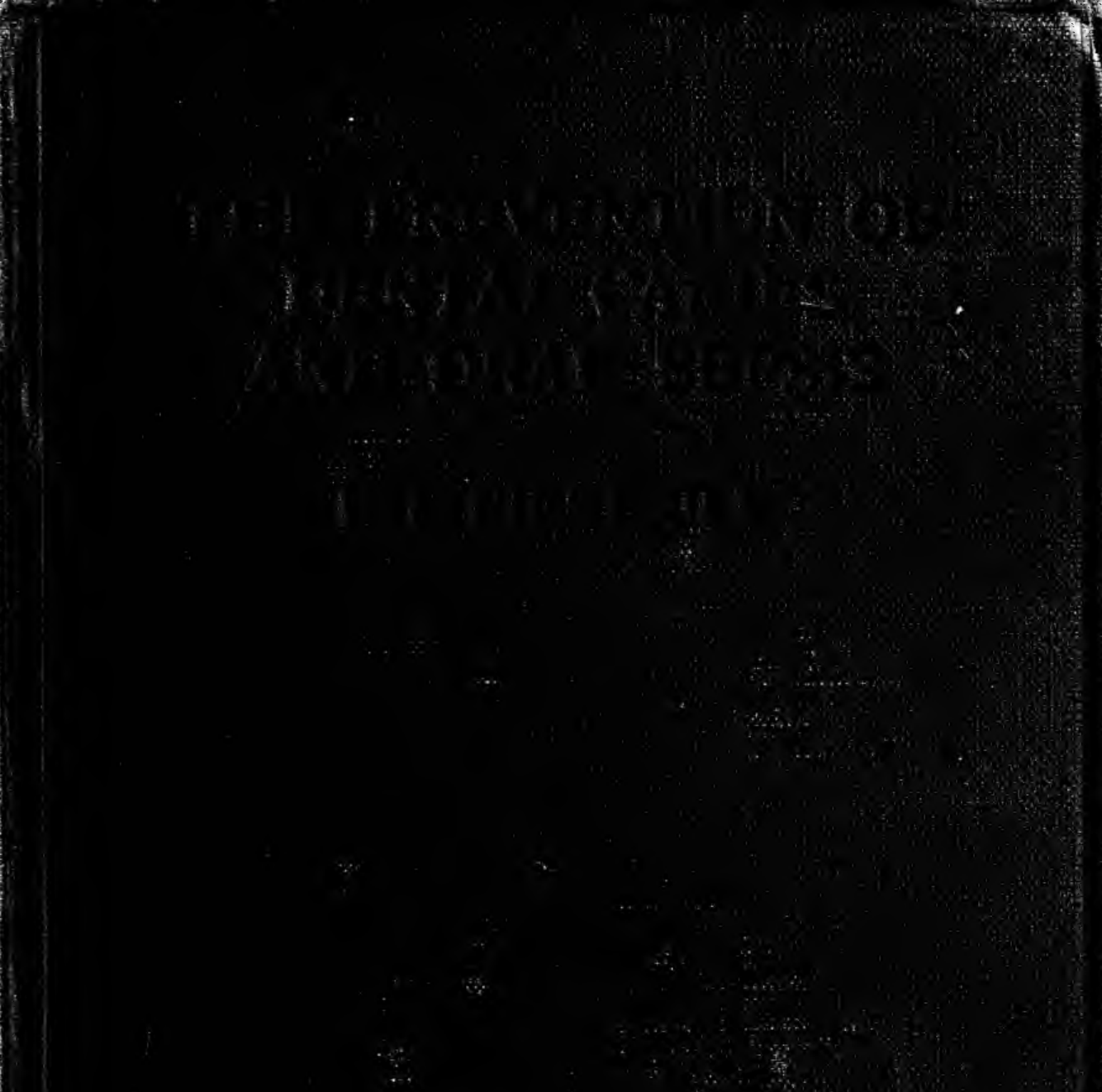

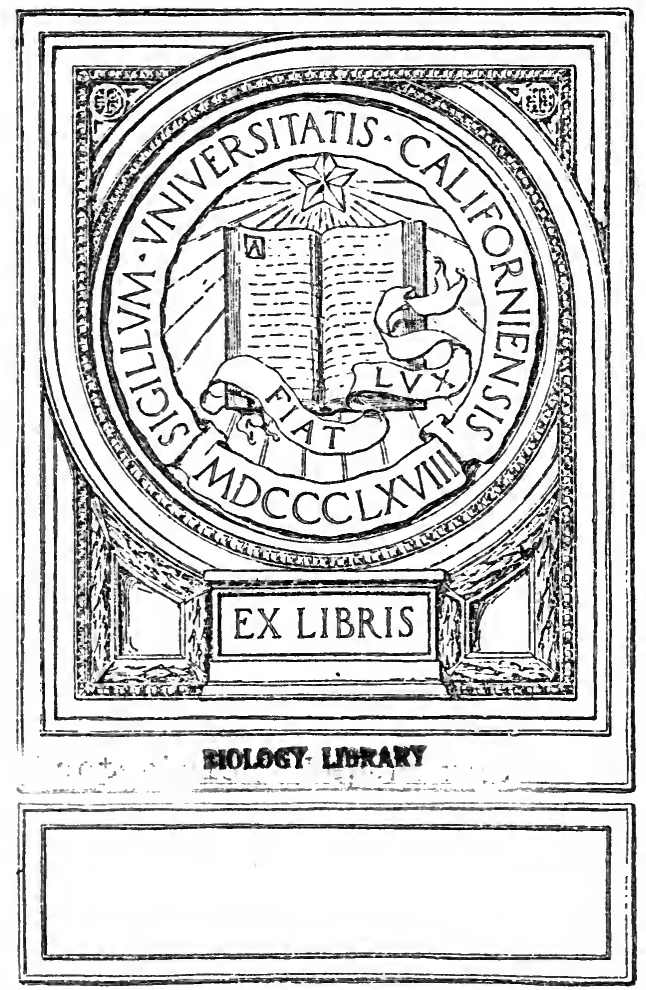


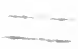

$\therefore=$ 
, 
THE PREVENTION OF DENTAL CARIES AND ORAL SEPSIS 


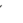




\section{THE PREVENTION OF DENTAL CARIES AND ORAL SEPSIS}

H. P. PICKERILL, M.D.

Ch.B. M.D.S. (BIRM.), L.D.S. (ENG.)

PROFESSOR OF DENTISTRY AND DIRECTOR OF THE DENTAL SCHOOL IN THE UNIVERSITY OF OTAGO

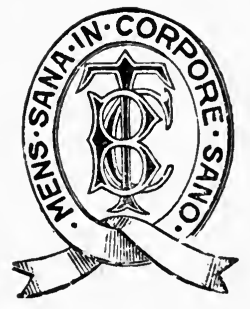

\section{LONDON}

B A.I LLIÈRE, TIN DALLAND COX 8, HENRIETTA STREET, COVENT GARDEN r9I 4 
11 K 331

75

? it

BIOLOET

inRART 
GILBERT H. BARLING, M.Sc., F.R.C.S.

DEAN OF THE FACULTY OF MEDICINE IN THE UNIVERSITY OF BIRMINGHAM

TO WHOM THE

INITIATION OF THIS WORK

WAS DUE 


\section{Digitized by the Internet Archive in 2007 with funding from Microsoft Corporation}




\section{PREFACE TO SECOND EDITION}

THE fact of the first edition being exhausted in the year following its publication has allowed but small opportunity for the insertion in the present edition of much that I had hoped to include, more especially in relation to work being done by others along similar lines in America, particularly by Drs. Gies, Lothrop, and Howe, and my own investigation into the cause of immunity in less civilized Maori children. Nevertheless, some fresh subjects have been broached in the present edition. These include accounts of our investigations into the bacteriology of the mouths of immune Maori children, the anatomy and physiology of mastication, thyroid insufficiency and dental caries, and the relationship between oral and other alimentary secretions. These subjects are dealt with in Chapters III., IV., XIII., and XVII. respectively. Also, additional experiments are described bearing on the solubility of the enamel surface in carbon dioxide, the neutralizing powers of tooth substance and saliva, the viscosity of saliva, the action of sulphocyanates in saliva, the digestion of starch, the effects of sugar-cane, white bread, and whole-meal bread on the teeth. Considerable additional evidence has been collected showing the lack of toughness or of fibrous elements in the food of races immune to caries. A number of fresh illustrations are also included.

This work has grown so largely since its inception that its original claim to be the R.C.S.E. Cartwright Prize Essay for IgIo can no longer be sustained.

My sincere thanks are due to my friend Mr. R. H. Pardoe for his invaluable assistance in seeing both editions through the press, and to the publishers for the courtesy and thoroughness with which the wishes of the author have been carried out under the somewhat difficult circumstance of his being fourteen thousand miles away.

H. P. P.

UNiversity of Otago, N.Z.

March, I9I4. 


\section{PREFACE TO FIRST EDITION}

WhEN this work was commenced nearly six years ago, a " plan of campaign" was drawn up to which it was decided to adhere, and to continue the work until the whole field had been covered, or at least investigated to some extent. It was further decided to publish nothing until the chain of evidence was more or less complete. This has obvious disadvantages, one of which is the length to which the subject has grown, and another the fact that in several matters the results have been forestalled in publication by others working along similar lines. The work, like all work, is still incomplete, but sufficiently important conclusions have been reached, I think, to justify publication.

The present volume is an amplified form of lectures which have been delivered in this University to Medical Practitioners and to Medical and Dental Students.

H. P. P.

November, I9II. 


\section{CONTENTS}

CHAPTER

I. HISTORICAL $\quad$ -

II. THE INCIDENCE OF DENTAL CARIES - $\quad$ - $\quad$ - 9

III. PATHOLOGY OF DENTAL CARIES - $\quad$ - $\quad$ - $\quad$ I 8

IV. THE ANATOMY AND PHYSIOLOGY OF MASTICATION - $\quad 36$

V. FACTORS OTHER THAN DIET INFLUENCING THE DISPOSITION OF THE TEETH AND LOWERING THEIR RESISTANCE TO CARIES -

VI. THE ENAMEL SURFACE: ITS PHYSICAL PROPERTIES AND POWERS OF RESISTANCE - $\quad$ - $\quad$ - $\quad$ - $\quad$ - $\quad$ - $7 \mathrm{I}$

VII. THE ENAMEL SURFACE: THE ODONTOGRAPH - $\quad$ - 82

VIII. THE ENAMEL SURFACE: ORIGIN AND SIGNIFICANCE OF THE

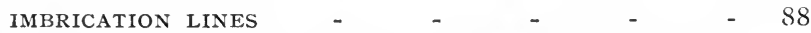

IX. THE ENAMEL SURFACE: DEPARTURES FROM THE “ NORMAL" STRUCTURE, AND THEIR PATHOLOGICAL SIGNIFICANCE - IOI

$\mathrm{X}$. PHYSICAL PROPERTIES OF THE ENAMEL SURFACE - II2

XI. CHEMICAL COMPOSITION OF ENAMEL - - - - I4I

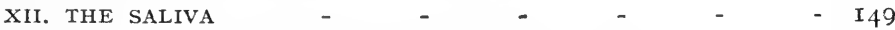

XIII. THYROID INSUFFICIENCY AND DENTAL CARIES - $\quad 232$

XIV. THE REDUCTION OF THE CARBOHYDRATE FACTOR BY PHYSIO

LOGICAL MEANS -

XV. THE REDUCTION OF THE BACTERIAL AND CARBOHYDRATE

FACTORS BY ARTIFICIAL MEANS - $\quad$ - $\quad$ - $\quad 265$

XVI. THE INOCULATION OF THE MOUTH WITH OTHER ORGANISMS 285

XVII. ALIMENTARY SECRETIONS CONSIDERED AS A WHOLE - 295

XVIII. THE DIET OF RACES RELATIVELY IMMUNE TO CARIES - 309

XIX. THE DIETARIES OF MODERN CIVILIZATION: THEIR ERRORS

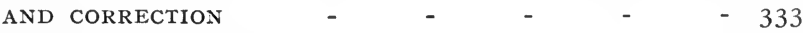

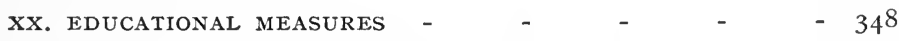

XXI. THE NEED FOR SPECIAL LEGISLATION $\quad-\quad-\quad-\quad 357$

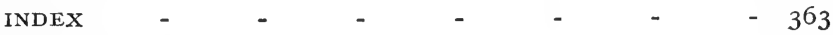

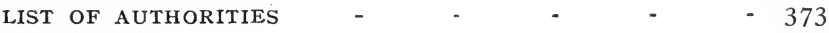





\section{LIST OF ILLUSTRATIONS}

FIG.

PAGE

I. DIAGRAM GRAPHICALLY REPRESENTING THE LIABILITY TO

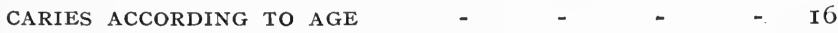

2. CURVE SHOWING REDUCTION IN ACIDITY - $\quad-\quad-22$

3. FILM OF BACTERIA ON ARRESTED CARIES - $\quad$ - $\quad 23$

4. FILM SHOWING THREAD-FORMS AND LEUCOCYTES - $\quad$ - 33

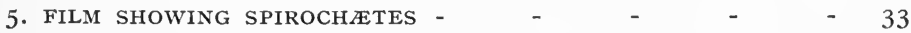

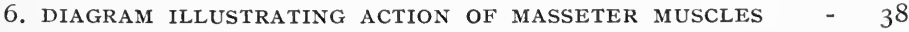

7. DIAGRAM ILlUSTRATING ACtion OF PTERYgoid MUSClES - 39

8. Diagram illustrating action of Mylohyoid muscles - 39

9. INTER-ARTICULAR INCLINED PLANES - $\quad$ - $\quad$ - $\quad$ - 4 I

IO. DIAGRAM ILLUSTRATING ACTION OF TONGUE IN TRITURATION 46

II. TRANSVERSE SECTION OF HUMAN TONGUE - $\quad$ - $\quad-50$

I2. TRANSVERSE SECTION OF SHEEP'S TONGUE - - - 5 -

I3. DIAGRAM OF RUBBER TEAT IN POSITION - $\quad$ - $\quad$ - 55

I4. SKIAGRAM OF A CHILD'S JAW AT FOUR AND A HALF MONTHS - 56

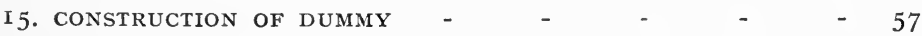

I6. CONTRACTION OF JAW: CARIOUS DECIDUOUS MOLAR - $\quad 59$

I7. DIAGRAM OF CONSTRICTED ARCH AND NASAL FOSS $Æ$ - 6 I

I8. DIAGRAM OF ACTION OF TONGUE IN STRAINEd DEGLUTition - 63

9. IMBRICATION LINES: MALACOTIC $\quad-\quad-\quad-\quad-75$

20. IMBRICATION LINES: MALACOTIC - $\quad-\quad-\quad-\quad 75$

2I. IMBRICATION LINES: MAORI - $\quad$ - $\quad-\quad-\quad-\quad-76$

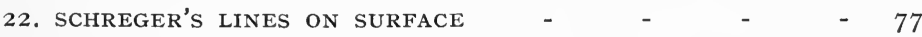

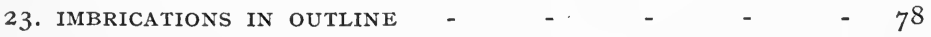

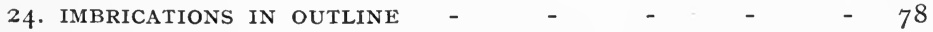

25. CURVATURE OF SURFACE: EUROPEAN AND MAORI TEETH - 79

26. CURVATURE OF SURFACE: PERMANENT AND DECIDUOUS TEETH 80

27. CURVATURE OF SURFACE: MOLAR OF DOG - $\quad-\quad$ - 80

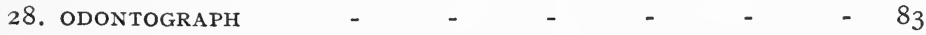

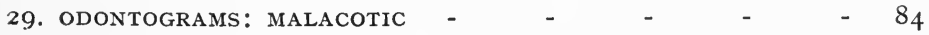

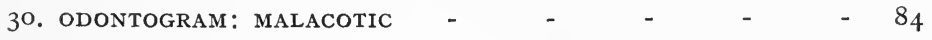

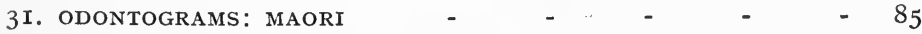

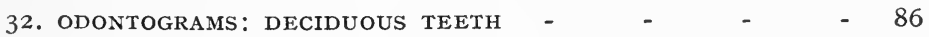

33. ODONTOGRAM: SCLEROTIC TOOTH $\quad$ - $\quad$ - $\quad$ - $\quad$ - 86

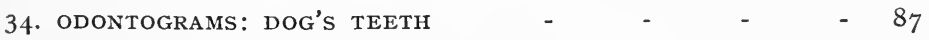

35. ARTIFACT LINES ON ENAMEL SURFACE $\quad$ - $\quad$ - $\quad$ - 89 
FIG.

36. IMBRICATIONS AND STRIE: CORONAL REGION

37. IMBRICATIONS AND STRIE: “ POINT OF CONTACT" REGION - 9 I

38. FILMS OF OVERLAPPING CHINESE WHITE: TRANSMITTED LIGHT 92

39. FILMS OF OVERLAPPING CHINESE WHITE: REFLECTED LIGHT - 93

4O. DIAGRAM OF ORIGIN OF IMBRICATION LINES - $\quad$ - 95

4I. DIAGRAM OF ORIGIN OF IMBRICATION LINES - $\quad$ - 96

42. VERTICAL SECTION OF HYPOPLASTIC TOOTH - $\quad$ - $\quad$ - 97

43. DiAgRAM ILLUSTRATING ABSENCE OF IMBRICATION LINES:

NATIVE AND DECIDUOUS TEETH - $\quad$ - $\quad$ - $\quad-98$

44. SKULL SHOWING CROWDING OF TEETH IN CRYPTS - $\quad 99$

45. SKULL SHOWING CROWDING OF TEETH IN CRYPTS - $\quad$ - 99

46. SKIAGRAM SHOWING CROWDING OF TEETH IN CRYPTS - IOO

47. IMBRICATIONS WITH CARIOUS CAVITIES - $\quad$ - $\quad$ - IO2

48. IMBRICATIONS: SCLEROTIC INCISOR - $\quad-\quad$ - $\quad$ - IO3

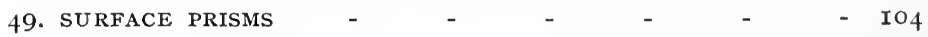

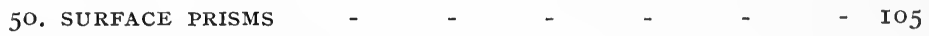

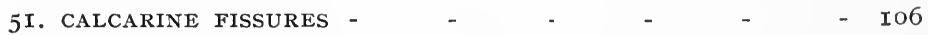

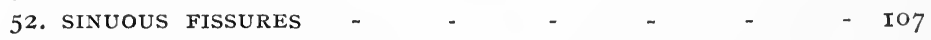

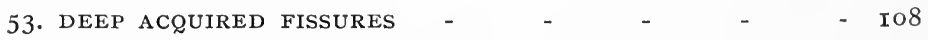

54. DEEP DEVELOPMENTAL FISSURES - - $\quad-\quad$ - $\quad-\quad$ - 109

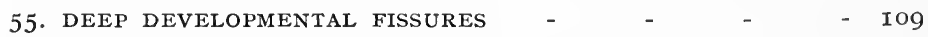

56. PENETRATION OF SILVER NITRATE: MALACOTIC TOOTH - II4

57. PENETRATION OF SILVER NITRATE: MAORI TOOTH - - II4

58. STAINING OF INTERPRISMATIC MATERIAL - - II6

59. PENETRATION OF SILVER NITRATE ALONG PRISMS - - II7

60. NON-PENETRATION OF ENAMEL BY SILVER NITRATE FROM

DENTINE -

6r. NON-PENETRATION OF ENAMEL BY SILVER NITRATE FROM

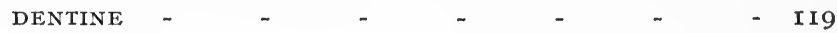

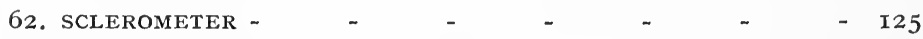

63. SEGREGATOR -

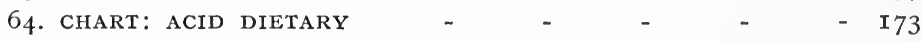

65. COLONIES ON AGAR CONTAINING SULPHOCYANATE O.05 PER CENT.

66. COLONIES ON AGAR CONTAINING SULPHOCYANATE O'OI PER

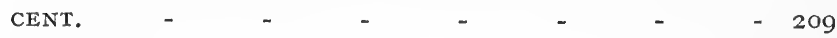

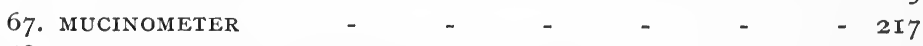

68. CURVE SHOWING REDUCTION IN VISCOSITY - - $\quad-220$

69. EPITHELIAL CELL IN SALIVA $\quad$ - $\quad$ - $\quad$ - 228

7O. PORTIONS OF THE CROWN AFTER SUBJECTION TO FER-

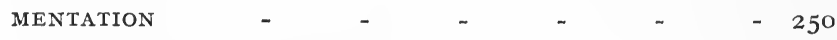

7I. SECTION OF A GRAIN OF WHEAT - $\quad$ - $\quad$ - $\quad-\quad 259$

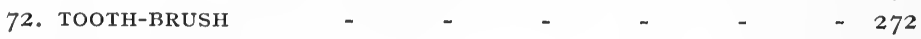

73. SACCHAROMYCES COAGULATUS - - $\quad-\quad-\quad 290$

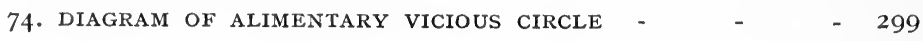




\section{TABLES}

SHOWING EFFECTS OF VARIOUS SALIVARY STIMULANTS

PAG E

I. AMOUNT AND ALKALINITY OF SALIVA: NATURAL STIMU-

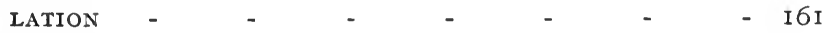

II. AMOUNT AND ALKALINITY OF SALIVA WITH FLAVOURED

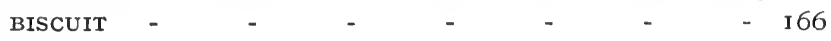

III. AMOUNT AND ALKALINITY OF SALIVA WITH ACIDS - $\quad$ - I69

IV. AMOUNT AND ALKALINITY OF SALIVA AFTER FLUIDS - I I I

V. AMOUNT AND ALKALINITY OF SALIVA DURING PROLONGED

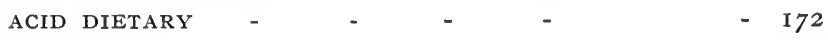

VI. AMOUNT AND ALKALINITY OF SALIVA AFTER DENTIFRICES - I76

VII. AMOUNT AND ALKALINITY OF SALIVA AFTER COCAINIZING

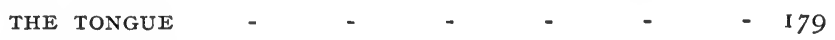

VIII. PTYALIN CONTENT OF SALIVA $\quad-\quad-\quad-\quad-\quad-192$

IX. SULPHOCYANATE CONTENT OF SALIVA - - - - 202

X. PHOSPhate CONTENT OF SALIVA - - - - $\quad$ - 2 II

XI. CHLORIDE CONTENT OF SALIVA - - - - - $\quad$ - 212

XII. MUCIN CONTENT OF SALIVA - $\quad$ - $\quad$ - $\quad$ - 216 



\section{INTRODUCTION}

Nearly one hundred years ago Fox wrote, as regards the prevention of dental caries, that "this delightful secret, although it is pretended to in the advertisement of every quack, we can only expect to acquire when the philosopher's stone and the grand panacea have been obtained." If this somewhat prophetic utterance is to be fulfilled, there would not seem to be any reasonable hope that the prevention of dental caries can yet be regarded as within the limits of practicability.

There is no doubt, though, that the disease has become much more serious in its proportions since Fox's time, and the attitude of fatalism towards it is no longer justifiable, and is not consonant with our modern knowledge of pathology and the means of immunity.

The general plan of the present work has been to inquire into past methods of the preventive treatment of dental caries, to compare these with present methods, and to collect data as to the incidence of caries in various nations and in various stages of civilization.

A critical examination of the powers of passive resistance on the part of the teeth has been carried out, together with an investigation of the forces of active natural protection, and a consideration of the means whereby both passive and active resistance might be fortified against the attacks of the oxygenetic or acid-forming forces as constituted in the combination of carbohydrates with organisms, and a further con- 
sideration of how these latter forces might be weakened or destroyed entirely.

The dietaries of " natural " and civilized peoples have been examined in detail, and inference from the one and corrections for the other have been made.

Finally, educational and legislative methods of dealing with the problem have been suggested. 


\title{
THE PREVENTION OF DENTAL CARIES AND ORAL SEPSIS
}

\author{
CHAPTER I \\ HISTORICAL
}

THE records of the practice of dentistry chiefly by medical means date back in the case of the Egyptians to somewhere about three thousand years before the Christian era. Even at this early period we have some evidence that methods were in vogue for the prevention of dental disease. For instance, the "Ebers" papyrus, probably compiled gradually from 3000 B.C. to I 550 B.C., contains prescriptions for " strengthening the teeth," of which the following is an example:

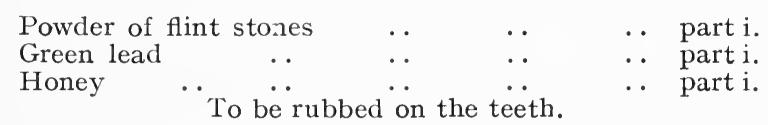

This might now be classified as an antiseptic and abrasive tooth-paste.

Records of Chinese medicine written about the same time as the above, and translated by Dabry, give prescriptions for whitening the teeth, and for a powder to prevent the progress of caries, the chief ingredients of which were musk, salt, and "Tsang-eul-tsee."

The writings of the early Greeks contain very little reference to dental hygiene, and as far as caries is concerned their attention seems to have been confined to relieving pain and 
hygiene of the "gums; yet how near Aristotle came to the modern conception of the cause of caries may be judged from this sentence taken from his book on "Problems":

"Why do figs, when they are soft and sweet, produce damage to the teeth? Perhaps because the viscous softness of the fig causes small particles to adhere to the gums and insinuate themselves into the dental interstices, where they very easily become the cause of putrefactive processes."

The only allusion made by Celsus to dental prophylaxis is that, " after rising, if it be not winter, the mouth should be rinsed with a quantity of fresh water." To be avoided in winter probably because of the teaching of Hippocrates, that "Cold is the enemy of the teeth."

Pliny states that the ashes of the head of a hare are a useful dentifrice, and if spikenard be added it will lessen the bad smell of the mouth. He also recommends burnt eggshells as a good tooth-powder.

Toothpicks of bone and metal seem to have been used commonly by the Romans. And it is stated by T. Wright* that Saxon women carried toothpicks of ivory in their chatelaines.

Messalina, the wife of the Emperor Claudius, we are told, used a dentifrice composed of calcined stag's horn, sal-ammoniac, and mastich of Chios. And several writers of this time mention that old (ammoniacal) urine was popularly used to preserve the teeth.

Paul of Ægina, during the seventh century, gives the following rules for the preservation of the teeth: All tainted food to be avoided, and also all possibility of indigestion and frequent vomitings; the use of very hard or glutinous food, or of such as may easily leave a residuum between the teethfor example, dried figs-and likewise very cold food and such as sets the teeth on edge, should also be avoided.

* "The Celt, the Roman, and the Saxon" (London, I852). 
He advises, too, that hard things should never be broken with the teeth, and that the latter be carefully cleaned, especially after the last meal of the day.

A representative of Arabian science, Avicenna, pointed out (about A.D. 1000) that very hard powders should be avoided, as these were likely to damage the teeth, and he recommends the use of burnt hartshorn.

During the fifteenth century, Giovanni of Arcoli drew up ten canons of dental hygiene which represented all the accepted teaching on the subject up to that time. The following is a brief summary of each canon:

I. Nothing must be eaten or done which would intertere with digestion.

2. Vomiting.

3. Sweet and sticky food.

4. Hard substances.

5. Food which sets the teeth on edge.

6. Food too hot or cold.

7. Leeks.

All to be studiously avoided.

8. Teeth to be cleaned with a piece of stick after every meal.

9. The mouth to be washed out afterwards with an aromatic decoction.

I0. A dentifyice to be used last thing at night or early in the morning.

It is with considerable surprise that one learns that so "advanced " a code of oral hygiene was advocated, and we may presume practised, over four hundred years ago, and to the significance of this fact and to the inferences to be drawn therefrom we shall have occasion to refer later on.

A century later (1580) we find Foreest of Leyden condemning the consumption of sweetmeats as harmful to the teeth.

Passing on to the seventeenth century, we have a somewhat 
different line of preventive treatment advocated by Rivière (I589-I655), who advised that the teeth should be cleaned with a small stick immersed in a mixture of "spiritus sulphuris aut vitrioli et mel rosatum," or, if the teeth were very dirty, in the acid undiluted. Unfortunately, no records are given of the results obtained by this somewhat heroic treatment.

Anton Nuck, a Dutch surgeon of the end of the seventeenth century, gives a formula for a tooth-powder much used in his time. The ingredients were powdered cuttle-fish, coralpowder, cream of tartar, Armenian bole, and powder of red roses.

During the next century we have Junker, Professor of Medicine at the University of Halle, recommending sage and also common salt to be rubbed on the teeth to preserve them from caries.

From Pierre Fauchard's book, "Le Chirurgien Dentiste," published in $\mathbf{1} \% \mathbf{2 8}$, we learn that tooth-brushes made of horsehair were in use at that time. He himself, however, condemns their use, and advises that the teeth should be cleaned with a fine sponge and tepid water to which has been added a fourth part of aqua vitæ. Instead of the sponge, the root of the marsh-mallow or lucern might be used. He also gives lengthy and complicated prescriptions for mouth-washes, the chief ingredients of which seem to have been aromatics, alcohol, gums, and sweetening agents. The same author makes the following very decided statement: "Little or no care as to the cleunliness of the teeth is the cause of all the maladies that destroy them."

Bunon in $\mathbf{1 7}^{\mathbf{1}} \mathbf{4 3}$, in an excellent work, pointed out the necessity of attention to cleanliness of the milk-teeth, and correctly stated the influence of disease of the latter over the permanent teeth.

At the end of the eighteenth century two erroneous theories gained ground, and did much to negative the teaching of 
dental hygiene. The first of these, shared alike by Hunter and Bell, was that caries originated from within, and was influenced little, if at all, by external agencies. The second was that, in order to keep the teeth free from "tartar," it was desirable that coarse and gritty dentifrices should be used, and the resulting denudation of the enamel of the teeth did not at all matter, since it would be developed afresh (presumably like nails or hair).

This latter theory was, however, strenuously opposed by Thomas Berdmore, dentist to King George III., who performed experiments to show the destructive effect of these toothpowders.

Fox in 1814 stated that " the principal means of preserving the teeth from decay consists in paying such a degree of attention to them in early life, during the period of the second dentition, as to allow the permanent teeth to acquire a proper regularity; and when these circumstances have been observed, young persons should be urged to keep their teeth very clean, and the daily use of a tooth-brush, with water only, will in most cases be quite sufficient. In addition to cleanliness, the habitual use of all kinds of food. at a moderate temperature will most certainly prevent any disease from taking place, unless there be any radical defect in the teeth themselves."

The methods adopted in America during the middle of the last century are given by Harris (1853) and Goddard (1854). The former, referring to experiments which had been done by Westcott ten years previously on the effect of various acids (including the acids produced by fermentation) on the teeth, states that he believes caries to be due more to acid oral secretions than to either acids introduced as condiments or tonics or formed by fermentation, and is of the opinion that if the functional operations of the body were properly performed, caries of the teeth would never occur. But he further states that it is necessary to artificially keep the teeth abso- 
lutely clean to avoid caries, and gives prescriptions for chalk dentifrices.

Goddard lays great stress upon perfect cleanliness and the frequent use of the toothpick and floss silk, and he gives various formulæ for antacid, detergent, and aromatic dentifrices.

Thus we are brought down to within measurable distance of the present time, and it will be convenient now to briefly summarize the methods we have already considered as having been used for the prevention of caries. We thus find that from the very earliest times--from the dawning of a higher civilization-soft sweet foodstuffs were recognized as a factor in the causation of dental disease (Aristotle); that very hard substances were to be avoided; that carbonate and phosphate of lime (as eggshells or burnt and powdered bones) were constantly prescribed as dentifrices; that alkalies in the form of ammoniacal salts were also frequently used and prescribed; that acids of all sorts were to be avoided; that " aromatics" were usually combined with dentifrices; that mechanical prophylaxis by means of toothpicks and frayed-out wood was in vogue from earliest times.

And all this before Pasteur had discovered the rôle of micro-organisms in the process of fermentation, or Lister had demonstrated the relationship of bacteria to disease and the method of eliminating them from diseased foci, or Miller had shown that caries of the teeth depends upon the formation of acids produced by the action of organisms on carbohydrate foodstuffs.

After these epoch-making discoveries, surely it would be thought that enormous strides would have been made in the preventive treatment of this particular disease; that the rationale of prophylaxis would have undergone a complete change; and that the incidence of caries would have been materially lessened. But has this been so? 
On all counts we are bound to answer in the negative. True, for some time after the discovery of antiseptics, such drugs were largely used in the mouth in order to endeavour to eliminate the bacterial factor; but it was soon found that it was impossible to sterilize the oral cavity, or even to decrease appreciably the number of organisms present for even an hour, without using drugs in such strength as could not be tolerated, on account of their disagreeable taste and deleterious effect on the oral mucous membrane. So that the routine use of antiseptics for this particular purpose has largely been given up.

What, then, are the means employed at the present time for the prevention of dental caries?

Naturally, they are numerous, and it is impossible here to review them in detail; but the two principles generally recognized may be gleaned from at least two widely accepted authorities. The British Dental Association, in its pamphlet on the preservation of the teeth (I905), advocates-

I. The use of a small tooth-brush with stiff bristles, used after the last meal.

2. The use of alkalies, such as soap, chalk, whiting, and also common salt.

3. The use of hard food.

Dr. Röse of Munich, like Giovanni of old, has drawn up a decalogue for the care of the teeth, in which he advocates-

I. Education of children and guardians in oral hygiene.

2. Mechanical cleansing of the teeth with a suitable brush or toothpick.

3. Avoidance of soft sweet foods.

4. Active mastication.

5. Alkaline and antiseptic dentifrices and mouth-washes.

Can it be possibly claimed that these measures differ in any fundamental principle from those which have been in the 
main practised ever since decay of the teeth has been treated by civilized man?

Compare the teaching of the nineteenth and twentieth centuries with that of the fifteenth, and it will be seen to be almost identical, differing only in one respect-the advocacy of " active mastication."

In view of the fact that the incidence of dental caries has not decreased, but increased, in geometrical ratio almost, during the last 500 years, and in view of the fact that a similar line of preventive treatment has been systematically carried out for the last five centuries, what inference are we entitled to draw?

A priori, it would seem obvious that the treatment was radically wrong; that the judgment of time and experience alike condemned it, or at least some important part of it; and that such measures have proved futile to arrest the progress of what has become the most prevalent disease of civilized communities.

\section{REFERENCES.}

Densham: Proceedings of the Royal Society of Medicine: Odontological Section, vol. ii., p. $7 \mathrm{I}$.

Henderson: British Dental Journal, vol. xxix., pp. 350, 392, 497.

HARRIS: Principles and Practice of Dental Surgery, I853.

Guerini: History of Dentistry, rgog.

Fox: Natural History and Diseases of the Human Teeth, I8I 4.

Goddard: Anatomy, Physiology, and Pathology, of the Human Teeth, I 854 . 


\section{THE INCIDENCE OF DENTAL CARIES}

\section{A. Racial.}

IT is now universally admitted that dental caries is to a great extent a disease accompanying civilization, and that its numerical incidence is largely proportionate to the state of civilization to which a particular race has attained. Nevertheless, it will be useful here to brieflyrecapitulate the evidence which has given rise to this opinion.

Magitot, ${ }^{1}$ examining the skulls in the Paris museums, found no caries of the teeth in the following races: Mexicans, Peruvians, Patagonians, aborigines of Australia, Madagascar, New Caledonia, the Malay Peninsula, or Java.

The now classical researches of J. R. Mummery ${ }^{2}$ into this question shed considerable light on the subject. The results of his investigation into the prevalence of caries in existing primitive races may be classified as follows:

\section{Race.}

Esquimaux ..

Maoris

Indians of N. W. American Coast

Fiji Islanders

Northern Hindoos

North American Indians

Eastern Polynesians ..

Southern Hindoos . .

Zulus

Sandwich Islanders .

Australians

Bushmen

Negroes (slaves)
Percentage of

Skulls showing

Carious Teeth.

$$
\mathrm{I} \cdot 4
$$

$3 \cdot 0$

$3 \cdot 9$

$5 \cdot 2$

$5 \cdot 9$

$9^{\circ} .5$

I I. 4

I $4^{\circ} \mathrm{O}$

I. $4 \cdot 2$

I9.O

$20 \cdot 5$

$20 \cdot 6$

$20 \cdot 8$ 
Such statistics as these, although tabulated here in the apparent order of prevalence, cannot be accepted as representing exactly the true state of affairs, since the number of skulls of each race examined varied considerably, and was in no case very large; neither is the proportion of sound to carious teeth given. Also, of course, the ages of the skulls in each race probably varied considerably.

Again, some in each race may have been Paleolithic, some Neolithic, and some from quite recent times.

Nevertheless, in spite of these possible sources of error, the tables may be taken, I think, as representing roughly the comparative prevalence of caries in the different races.

On comparing the incidence of caries in uncivilized races with that in civilized races of the present time, a vast difference is observed. The Physical Deterioration Committee reported in $\mathrm{IgO}_{4}$ that " there is no doubt that the teeth of the people have become much worse of late years, and in many parts of the country may now be described as very bad." There can be no doubt that these words do not err on the side of exaggeration, as the following statistics will prove:

\begin{tabular}{|c|c|c|c|}
\hline Class of Person examined. & $\begin{array}{l}\text { Number } \\
\text { of Persons } \\
\text { examined. }\end{array}$ & $\begin{array}{l}\text { Percentage } \\
\text { of Persons } \\
\text { having } \\
\text { Carious } \\
\text { Teeth. }\end{array}$ & Investigators. \\
\hline $\begin{array}{l}\text { English and Scotch } \\
\text { school boys and girls }\end{array}$ & 10,500 & $86 \cdot 00$ & $\begin{array}{l}\text { British Dental As- } \\
\text { sociation }\end{array}$ \\
\hline $\begin{array}{c}\text { Residents of Baden and } \\
\text { Hamburg }\end{array}$ & 一 & $\left\{\begin{array}{l}98 \cdot 75 \\
96 \cdot 40\end{array}\right\}$ & Röse 3 \\
\hline Halifax children $\quad \ldots$ & - & $95^{\circ} 00$ & M.O.H. for Halifax ${ }^{3}$ \\
\hline Leith school-children .. & 270 & $98 \cdot 60$ & Robertson ${ }^{4}$ \\
\hline Kettering children & 5,724 & $93 \cdot 00$ & Layton ${ }^{5}$ \\
\hline $\begin{array}{l}\text { Birmingham school- } \\
\text { children }\end{array}$ & I, 424 & $94 \cdot 70$ & Richards $^{6}$ \\
\hline Cambridge Dental Clinic & $\mathrm{I}, 403$ & $96 \cdot 50$ & Cunningham ${ }^{7}$ \\
\hline $\begin{array}{l}\text { School-children of North- } \\
\text { ern Germany }\end{array}$ & 19,725 & $95^{\circ} \mathrm{OO}$ & $\begin{array}{l}\text { Schleswig-Holstein } \\
\text { Dental Association }\end{array}$ \\
\hline
\end{tabular}

These figures show a quite remarkable uniformity. 
The British Dental Association's report is, I believe, less recent than the others; so that this would tend to show that even in quite recent years the number of persons affected with caries is increasing.

It is also of interest and some importance to compare the increase in the prevalence of caries which has occurred in individual races.

Mummery, investigating the number of Ancient British and Anglo-Saxon skulls showing carious teeth, gives the following figures:

British skulls of the Bronze Age showed carious teeth in .. $2 \mathrm{r} \cdot 8$

Romano-British skulls showed carious teeth in .. $\quad . .32 \cdot 0$

Anglo-Saxon skulls showed carious teeth in $\ldots . \quad \ldots \quad$ I $5^{\circ} \mathrm{O}$

Wright ${ }^{8}$ examined the Driffield Collection of Skulls from the barrows of the East Riding of Yorkshire. They belonged to the Bronze and Early Iron Ages. The percentage of actual caries was only $I^{\prime} 5$. Wright, in the communication referred to, attributed "alveolar abscesses to caries," and this, of course, made the percentage much higher; but, from my own experience in the examination of skulls and teeth of uncivilized races, I think that such an inference was probably erroneous, since alveolar abscesses result in such races most commonly through an opening up of the pulp cavity due to excessive attrition, without the slightest trace of caries.

An examination of 562 skulls from long barrows gave 8 per cent. of caries, and 47 skulls from round barrows showed 2I per cent.

In an examination of 260 Maori skulls-all from an uncivilized age- - I found carious teeth present in only two skulls, or 0.76 per cent.

By taking the average of Mummery's and my own investigations, the incidence of caries in the Maori is found to be $\mathrm{I} \cdot 2$ per cent. in a total of 326 skulls. This is lower even than 
the Esquimaux, and shows the Maori to have been the most immune race to caries, for which statistics are available.

Comparing these figures with those applicable to the present time, we find that the descendants of the Britons and AngloSaxons are afflicted with dental caries to the extent of 86 per cent. to 98 per cent. ; and after examining fifty Maori schoolchildren living under European conditions entirely, I found that 95 per cent. of them had decayed teeth.

It will be of interest now to compare the rercentage of carious teeth found in uncivilized races and in the higher civilized races of to-day.

Patrick" has examined all the "prehistoric crania to be found in the various museums in America." The results are as follows:

\section{Race.}

Asiatics (including Malays, Chinese, Japanese,

Armenians, Hindoos, and Burmese) ..

Egyptians and Africans $\quad \ldots \quad \ldots \quad \ldots$

Polynesians and Australians .. $\quad . . \quad$..

Central Americans $\quad \ldots \quad \ldots \quad \ldots \quad \ldots \quad \ldots$

North Americans (including Esquimaux) . .

South Americans (including Tierra del Fuegians and Guanches) $\quad . . \quad \ldots \quad \ldots$

Europeans (including " a few modern

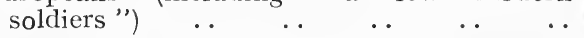

Number of Teeth examined.

Percentage of Carious Teeth.

2,180

$2 \cdot 064$

3,306

$2,73^{8}$

930

27,362

$3 \cdot 418$

$4 \cdot 309$

$4 \cdot 872$ $5^{\bullet} 093$

$\begin{array}{l:l}6.719 & 5 \cdot 80_{4}\end{array}$

$3422: 7 \cdot 079$

Comparing these low percentages of carious teeth with those obtaining at present, we find that Ottofy, ${ }^{10}$ after an examination of 14,644 teeth of American school-children, reported that 30 per cent. were carious. Cunningham, ${ }^{7}$ reporting on the work of the Cambridge Dental Clinic, gives statistics which show that $52 \cdot 3$ per cent. of the teeth of the children examined $(I, 403)$ were carious.

In an examination of two schools of Maori children living 
under civilized conditions, I found that ${ }^{5} \cdot 6$ per cent. of the teeth were carious.

Very few statistics of this nature are available, and it is a matter for regret that, when examinations are made, the number of sound, as well as of carious, teeth in each mouth is not tabulated more frequently; for, after all, this is by far the most important and serious aspect of the present prevalence of this disease.

If the incidence of caries per head of the population were Ioo per cent., but if only 5 per cent. of the total number of teeth were affected, there would be but little work for the dental profession and no occasion for the present argument.

Summing up the results of these investigations, we find-

I. That the number of persons affected with dental caries who live under uncivilized or "natural" conditions is comparatively small, varying from I to 20.8 per cent., whilst in civilized modern races the percentage is as high as 98 per cent., the increase being at least $77 \cdot 4$ per cent.

2. That the number of teeth affected with caries in each individual is far less in those leading natural lives than in those leading artificial or highly civilized lives, in the former varying from 2 per cent. to 7 per cent., whilst in the latter it has become as high as from $I_{5}$ per cent. to 52 per cent., showing a maximum increase of 45 per cent.

3. That in the British race, which has been subject to the influence of civilization for nearly 2,000 years, the increase in the percentage of caries is about 79 per cent. (calculated from the averages of the statistics given above). This corresponds closely to the difference in percentage given above in No. I.

4. That in the Maori race, which has been subject to the influences of civilization for only seventy or eighty years at the very most, but has only become "civilized" in habits 
quite recently, the increase is one of 93 per cent. (But against this apparently high figure has to be set the fact that the incidence of caries in each mouth is comparatively low.)

Reviewing now all the above investigations, we are clearly justified in our original statement that there is a distinct relationship between the incidence of caries and the state of civilization. An interesting example of this is found in the fact that the prevalence of dental caries apparently fell in England after the invasion by the Anglo-Saxons-a race less civilized than the Romanized Britons whom they displaced.

Civilization, however, per se, cannot be the cause of so much difference in the incidence of caries. For instance, the Maori was undoubtedly a more "civilized" and a higher type of savage than the Zulu, the Esquimaux than the Bushmen, and the North American Indians than the Australian aborigines; yet in each of these cases the race of higher type showed the least incidence of caries.

It must be, then, some particular, though not always essential, accompaniment of civilization which is responsible for the increase. Naturally, one would expect that factor of civilization which particularly affected the function of the teeth to be responsible, and this undoubtedly is so to a very large extent. The function of the teeth is primarily to masticate food, and there can be no doubt that in those races in which this function is most perfectly carried out, and in which the teeth have the most natural work to do, dental caries is correspondingly rare. In fact, it would be more correct to say that the numerical incidence is largely proportionate to the state of " civilized" diet to which a particular race has attained. The physical, chemical, anatomical, and physiological effects of this dietetic evolution on the teeth will be considered in detail subsequently. 


\section{B. Sexual.}

The opinion seems to be unanimous that females are slightly more liable to caries than males. Statistics vary on the point. Magitot gives the difference as 25 per cent. in favour of males. Röse gives the percentage of caries in boys as 26 , and in girls $26 \cdot 2$. Greve $^{11}$ states that 3 per cent. more girls than boys are affected with caries.

\section{Age.}

Since statistics bearing upon the incidence of dental caries are almost entirely based upon the examination of schoolchildren, it is difficult to give any precise data as to the percentage of incidence in later life, but in general terms it may be stated that caries is essentially a disease of adolescence. It is a matter of common observation that the progress of the disease in any particular mouth is much slower, and even may be altogether arrested, after the patient has attained to adult life. Or, expressed otherwise, if a tooth remains free from decay for some years-say eight to ten-after eruption, there is a strong probability that it will remain immune for at least a prolonged period. This is an important fact, and will be referred to later.

The accompanying diagram (Fig. I), drawn up by Mr. F. W. Richards, illustrates graphically the rapid increase in incidence of caries between the ages of six and thirteen. It illustrates not only the fact that the number of carious teeth increases, but also-more serious-the much increased extent of the disease in each tooth.

Cunningham's figures show a similar increase, but the incidence of caries is much higher throughout. Thus, at three years old the percentage of children having carious teeth is 84 . This increases steadily up to the fourteenth year, when the number reaches nearly Ioo per cent. 
Greve's statistics, however, do not agree with the foregoing. He gives 93.3 per cent. at six years, $96 \cdot 5$ per cent. at ten, and

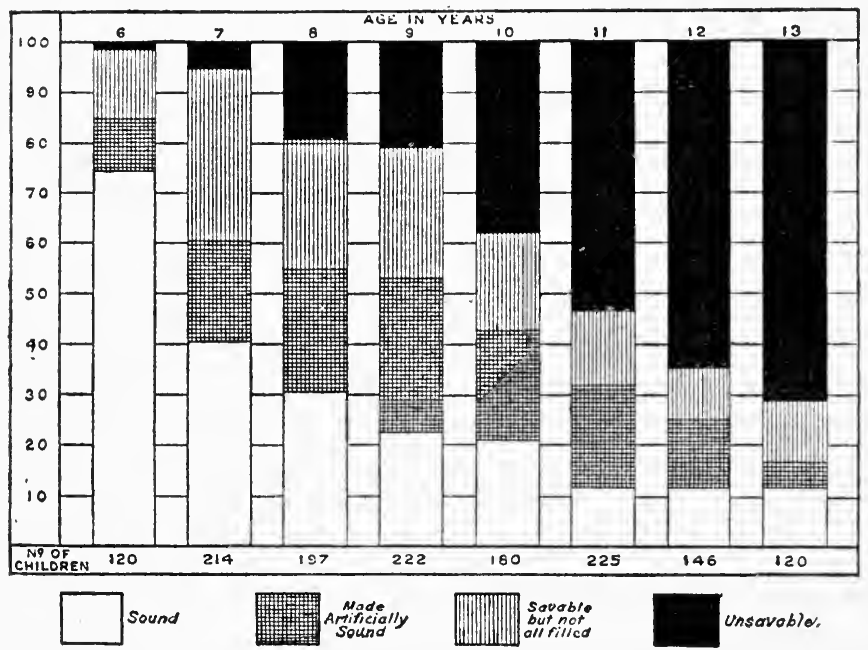

Fig. I.-Diagram graphically Representing the Relative Liability to Caries acCording to Age.

(By permission of the "British Dental Journal.")

94.5 per cent. at fifteen. Evidently, caries of the deciduous teeth is very prevalent amongst German children.

\section{Susceptibility of Different Teeth.}

The report of the work of the dental surgeons of the United States Army for the years IgOI-IgO3 affords the most valuable information on this point, since over 70,000 teeth were treated by fillings and extractions. It is not proposed to give here the percentages of each tooth treated. It will be sufficient for our purpose to give the more important groups: 
Teeth.

First permanent molars Second permanent molars Upper central incisors Premolars Third molars Upper canines Lower incisors Lower canines

\begin{tabular}{lllc} 
& & \multicolumn{2}{c}{$\begin{array}{c}\text { Average Percentage } \\
\text { of Each Carious. }\end{array}$} \\
ars & $\ldots$ & $\ldots$ & $6 \cdot 5$ \\
olars & $\ldots$ & $\ldots$ & $5 \cdot 1$ \\
$\ldots$ & $\ldots$ & $\ldots$ & $3 \cdot 9$ \\
$\ldots$ & $\ldots$ & $\ldots$ & $2 \cdot 8$ \\
$\ldots$ & $\ldots$ & $\ldots$ & $2 \cdot 0$ \\
$\ldots$ & $\ldots$ & $\ldots$ & $1 \cdot 7$ \\
$\ldots$ & $\ldots$ & $\ldots$ & $0 \cdot 7$ \\
& $\ldots$ & $\ldots$ & $0 \cdot 5$
\end{tabular}

Average Percentage

Hence it is obvious that certain teeth are, by reason either of their position or function, far more susceptible than others, the first molars being more than twelve times more susceptible than the lower canines, for instance, and the upper incisors five times as susceptible as the corresponding lower teeth.

Another important fact borne out by this report is the increased susceptibility of upper teeth. Forty-three thousand upper teeth were carious, but only 26,000 lower, or nearly twice as many upper as lower teeth.

This confirms Hitchcock's figures, ${ }^{12}$ compiled from 20,000 cases of fillings and extractions. He found I3,I36 upper teeth and 6,864 lower teeth carious-again a proportion of nearly two to one.

Magitot, however, gives the proportion as three to two in an analysis of 10,000 cases.

\section{REFERENCES.}

1 Magitot: Marshall's Operative Dentistry, p. I22.

2 Mummery, J. R.: Transactions of the Odontological Society, vol. ii., New Series.

3 Röse and British Dental Association Report, quoted by Smale and Colyer in "Diseases and Injuries of the Teeth," p. 242.

4 Robertson: British Dental Journal, I909, p. 655.

5 Layton: British Dental Journal, I908, p. 865.

${ }^{6}$ Richards: British Dental Journal, I909, p. 75I.

7 Cunningham: British Dental Journal, I908, p. 872.

8 Wright, W.: British Dental Journal, r903, p. 57.

9 PATRICK: Marshall's Operative Dentistry, p. I2I.

10 OtтоFy: Marshall's Operative Dentistry.

11 GREVE: Cor. Blatt. für Zahn., July, I 899.

12 Hitchсоск: Wedl's Pathology of the Teeth. 


\section{PATHOLOGY OF DENTAL CARIES}

IT would serve no useful purpose here to review in detail all the theories which have been at various times put forward to account for the phenomena of dental caries. It will be sufficient to briefly mention the more important ones.

Hippocrates advanced the theory that caries, in common with all other diseases, was due to a disturbance of the four principal humours of the body, and this theory was not extinct even as late as I757.

The belief that the disease was a vital process, was in reality a form of inflammation similar to caries of bone, has been held by a large number of celebrated authorities from Galen down to Hunter and Fox, and Abbott in I879 strongly supported the theory.

In the Middle Ages the worm theory of caries was much in vogue, and quacks (and also physicians) used various fumigations for expelling the "worms "- a method still practised by quacks in some parts of Italy. More modern theories are-

I. The Electrical Theory, enunciated by Bridgeman, in which each tooth is supposed to act as a small galvanic battery, setting up currents which hydrolyze any stagnating food débris, thus setting free acids which produce caries.

2. The Chemical Theory. - That acids were destructive to the teeth has been recognized from quite early times, and Westcott in I843 showed that acids, whether organic, inor- 
ganic, or produced by "fermentation," might dissolve the enamel, and so cause caries. Harris (I853) stated his belief that caries was caused by acidity of the oral secretions, and Sir J. Tomes (I873) ascribed the cause of decay to the process of fermentation and acid-production.

3. The Septic Theory, advanced by Milles and Underwood, in which caries is said to be due to the action of acids and the action of germs, the germs secreting the acid from the protoplasm of the organic fibrils upon which they live.

4. It remained, however, for Miller (I885) to advance what is now known as the Chemico-Parasitic Theory, which alone satisfactorily accounts for all the phenomena of caries of the teeth. This theory is almost universally accepted at the present time, and it is accepted as the pathological basis of the author's investigations to be hereafter described. The important points in the chemico-parasitic theory are-

(I) That the organisms of the mouth, by the secretion of an enzyme (or by their own metabolism), so act upon carbohydrate food material as to form acids by a process of fermentation. The chief acid formed is lactic, but butyric, acetic, formic, succinic and other acids may also be formed.

(2) Carbohydrate food material lodging between or on the teeth is the source of acid, which attacks the lime salts of the enamel, dissolving the interprismatic cement substance. Thus by the action of the acid and by the force of mastication the enamel is destroyed or weakened, and removed mechanically.

(3) The action of the micro-organisms of the mouth upon protein material is to form an excess of alkaline substances that have no action upon the enamel other than a beneficial one.

(4) The enamel being penetrated, the solution of the lime salts of the dentine is brought about in the same manner, the organisms penetrating along the dentinal tubules. 
(5) The further stages of caries of dentine is brought about by another set of organisms which secrete a proteolytic enzyme. This dissolves the collagen of the dentine matrix, thus forming a cavity.

Goadby* has classified the organisms concerned in the production of caries as follows:

\section{Acid-forming Bacteria.}

I. Streptococcus brevis

2. B. necrodentalis

3. Staphylococcus albus

\} Deep layers of carious dentine.

I. Streptococcus brevis

2 Sarcina lutea

3. Sarcina alba (Eisenberg)

4. Staphylococcus albus

5. Sarcina aurantica

6. Staphylococcus aureus

Superficial layers of carious dentine.

\section{Bacteria which liquefy Dentine (Decalcified.)}

(None isolated) .. $\quad$. $\quad$. . Deep layers of carious dentine.

I. B. mesentericus ruber

2. B. mesentericus vulgatus

3. B. mesentericus fuscus

4. B. furvus

5. B. gingiva pyogenes

๑. B. fluorescens liquefaciens motilis

7. B. subtilis

Superficial layers of carious dentine.

The following equations express briefly the phenomena of lactic-acid fermentation as it takes place in the mouth:

Monosaccharides :

$$
\underset{\text { Glucose. }}{\mathrm{C}_{6} \mathrm{H}_{12} \mathrm{O}_{6}}=\underset{2}{2\left(\mathrm{C}_{3} \mathrm{H}_{6} \mathrm{O}_{3}\right)} \text { Lactic acid. }
$$

Disacchavides :

$$
\begin{aligned}
& \mathrm{C}_{12} \mathrm{H}_{22} \mathrm{O}_{11}+\mathrm{H}_{2} \mathrm{O}=\mathrm{C}_{6} \mathrm{H}_{12} \mathrm{O}_{6}+\mathrm{C}_{6} \mathrm{H}_{12} \mathrm{O}_{6} \text {. } \\
& \text { Cane-sugar. Dextrose. Lævilose. } \\
& \mathrm{C}_{6} \mathrm{H}_{12} \mathrm{O}_{6}=2\left(\mathrm{C}_{3} \mathrm{H}_{6} \mathrm{O}_{3}\right) \text {. } \\
& \text { latctic acid. }
\end{aligned}
$$


Polysaccharides:

$$
\begin{aligned}
& \left.\underset{\text { Starch. }}{\mathrm{C}_{6} \mathrm{H}_{10} \mathrm{O}_{5}}\right)^{3}+\mathrm{H}_{2} \mathrm{O}=\underset{\text { Dextrin. }}{\mathrm{C}_{6} \mathrm{H}_{10} \mathrm{O}_{5}}+\underset{\mathrm{H}_{2} \mathrm{H}_{22} \mathrm{O}_{11}}{\mathrm{C}_{2}} . \\
& \mathrm{C}_{12} \mathrm{H}_{22} \mathrm{O}_{11}+\mathrm{H}_{2} \mathrm{O}=2\left(\mathrm{C}_{6} \mathrm{H}_{12} \mathrm{O}_{6}\right) . \\
& 2\left(\mathrm{C}_{6} \mathrm{H}_{12} \mathrm{O}_{6}\right)=\underset{4}{\mathrm{H}_{4}\left(\mathrm{C}_{3} \mathrm{H}_{6} \mathrm{O}_{3}\right) .} \\
& \text { Lactic acid. } \\
& 2\left(\mathrm{C}_{3} \mathrm{H}_{6} \mathrm{O}_{3}\right)=\underset{\text { Butyric acid. }}{\mathrm{C}_{4} \mathrm{H}_{8} \mathrm{O}_{2}+{ }_{2} \mathrm{CO}_{2}+2 \mathrm{H}_{2} .}
\end{aligned}
$$

This shows that monosaccharides are directly fermentable, and that disaccharides require to be first inverted to monosaccharides by an enzyme formed by certain of the mouth organisms before lactic acid is produced. Starches require a double inversion-the first stage being brought about either by ptyalin or organisms-before fermentation to an acid can occur; but in all cases it is seen that lactic acid is the end product, unless, as occasionally happens, this lactic acid is again split by the action of organisms to yield butyric acid.

It is, however, necessary to state, since the fact does not appear to have been sufficiently considered in the past, that: in the production of caries in the mouth we are not dealing with organisms and carbohydrate media only, for these are always admixed with a variable quantity of saliva of differing composition, and this third factor is capable of considerably modifying the resulting action.

Perhaps the most convincing proof of the validity of this theory is the fact that in accordance with it caries can be artificially produced in the laboratory so as to resemble in its microscopical features all the phenomena of natural caries. For this purpose it is only necessary to take a portion of such food as would constitute an ordinary meal (carbohydrates preponderating), inoculate it with saliva, place a tooth whole or in section in the mixture, and incubate. In twenty-four hours the mixture will be found to be markedly acid. The acid continues to increase daily, and may under these circum 
stances become so strong as to destroy the acid-forming organisms. To avoid this, a little sodium hydrate or carbonate should be occasionally added. In about fifty days the teeth

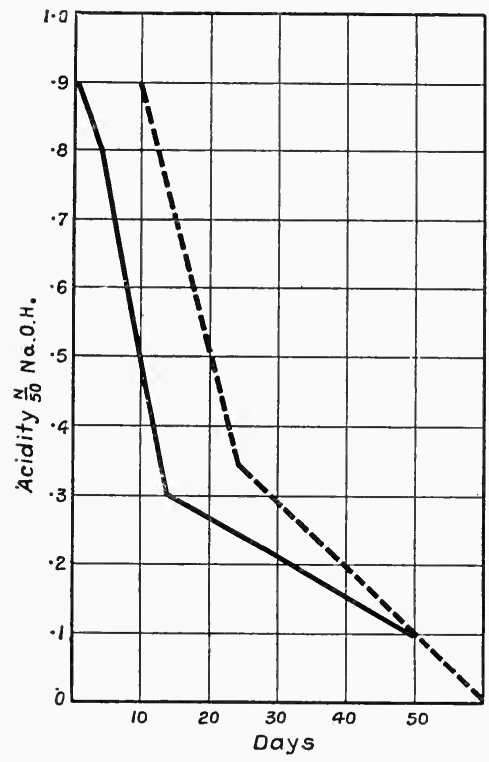

Fig. 2.-Curve showing ReducTION IN THE ACIDITY OF LACTIC ACID PRODUCED BY ITS ACTION on Tooth Substance.

Whole teeth.

-...- Tooth fragments. may be removed and examined, with the result as above stated.

Does lactic acid combine with the lime salts of the teeth, and is it thereby neutralized?

It has been suggested that this does not occur, but the experience of the author is contrary to this, as may be indicated by the following experiments :

I. Teeth (malacotic) were placed in 150 c.c., 0.5 per cent. lactic acid, the acidity of which to litmus was 0.9 per c.c. $\left(\frac{\mathrm{N}}{50} \mathrm{NaOH}\right)$, and in fifty days the acidity was reduced to $O \cdot I$ per c.c. The teeth were at first rapidly decalcified, but the effect on fresh malacotic teeth, suspended in the same solution after the thirtieth day for the remaining twenty days, was barely perceptible.

2. Fragmented teeth were placed in a similar solution. The decrease in acidity was quite similar, but progressed a little further; in fifty days it was nil.

The accompanying chart will show the results at a glance.

It may be said, therefore, that, although the action is a slow one, tooth substance does neutralize lactic acid. 
It is contended by Leon Williams and Black that the essential feature in the pathology of caries is the presence of bacterial plaques upon the surface of the teeth, and that the lactic acid is secreted as such by the organisms themselves. Since this has a bearing upon the preventive treatment of caries, it is necessary to consider the importance of this claim.

I. The presence of plaques is undoubted, but they occur on all surfaces of teeth, and not solely on the mesial and distal

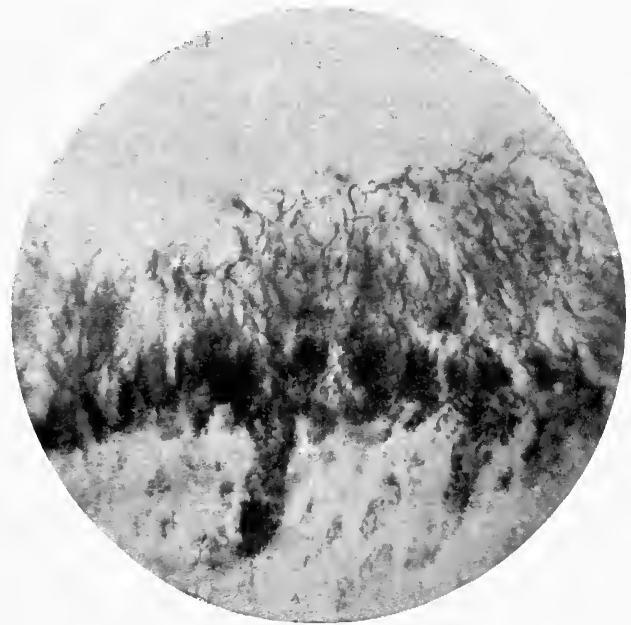

Fig. 3.-Bacterial Film on the Surface of Arrested Caries of Dentine.

surfaces, which are most liable to attacks of caries. They occur on the teeth of immune people apparently as frequently as upon the teeth of susceptible persons. They are found upon animals' teeth; they are found upon the inner surface of secondary enamel decay.* A bacterial film may be demonstrated, too, upon the surface of teeth in which the process of caries has been naturally arrested and the dentine become

* Niller, Dental Cosmos, 1902, p. 437. 
quite hard, where, if anywhere, one would expect an acidforming film of bacteria to be absent (see Fig. 3).

2. If the film of organisms is the essential origin of the acid, it would tend to constantly remove itself by dissolving its base of attachment.

3. The film of organisms cannot produce acid from Nasmyth's membrane nor from the oral secretions. They must have a supply of carbohydrate medium for the purpose, and this must remain in apposition for a sufficient length of time for enough acid to be "secreted."

4. The saliva, which almost immediately permeates all food upon mastication, contains millions upon millions of organisms capable of transforming carbohydrates into acids. Why is it then necessary to look to any particular colonies for the formation of that acid?

We therefore come to the conclusion (I) that bacterial plaques upon the teeth are not an essential factor in the causation of caries; (2) that the lodgment and infection of carbohydrate material is the essential factor, and that those circumstances which favour the formation of bacterial plaques upon the teeth also favour the lodgment of carbohydrates, and so the occurrence of the two usually coincides.

Therefore it is evident that the two essential attacking forces in this disease are (I) the acid-forming organisms of the mouth, (2) the presence of fermentable carbohydratesthe first active, the second passive.

The inception and progress of the disease are, however, by no means so simple as would appear from the above statements and equations. The phenomena are profoundly modified by a large variety of factors, such as the character and number of organisms present, the variety and amount of carbohydrate material available for acid production, the amount, alkalinity, and diastatic action of the saliva, the resistance of 
the enamel surface, the shape and development of the jaws and the disposition of the teeth. And since these conditions vary in different individuals, it is evident that the possible permutations and combinations of the various factors are very numerous and complex. In fact, dental caries is similar to many other diseases. It is not the "effect" of any single "cause," but it is the resultant of several forces-it may be few or many, and not always similar-acting in one general direction, and which happen for a longer or shorter period to be coincident.

\section{Dental Caries as a Source of Oral Sepsis.}

During the first stage of dental caries, when the enamel only is being dissolved by means of lactic acid, it can scarcely be said that sepsis, in the ordinary sense of the word, is present; but as soon as the dentine becomes involved in the morbid process there can be no doubt that sepsis is present-sepsis, but not suppuration.*

The proteins of the fibrils and dentinal matrix are being metabolized largely by pyogenic organisms giving rise to poisonous end-products-gases, such as carbon dioxide, sulphuretted hydrogen, indol, and skatol, and liquid toxins, albumoses, etc., all of which must be swallowed by the patient. Before the pulp is actually exposed, it is frequently infected by organisms passing down the dentinal tubules, and suppuration, either localized or general, occurs in the pulp without there being any external evidence of its presence; the products of this suppuration must, of course, be wholly absorbed by the lymph and blood streams. A stage further, and the pulp is exposed, and sooner or later becomes gangrenous-septic in the highest sense of the word-and

* The term " sepsis" is now used so frequently as being synonymous with suppuration that we lose sight of its real meaning $(\sigma \dot{\eta} \pi \omega=\mathrm{I}$ make putrid), and the destruction of dentine is really a putrid process. 
again all the gangrenous products are swallowed by the patient.

Proteins are decomposed by mouth organisms, firstly into alkaloidal substances, and secondly, in the presence of air, into simpler decomposition products.

In the absence of free oxygen, however, such as in infected pulps without exposure, the process does not proceed further than the alkaloidal stage. These, for the most part poisonous, alkaloids are then absorbed, and produce a condition of toxæmia.

In the final stages the periodontal tissues become infected through the apical foramen, suppuration occurs, and an alveolar abscess develops, which, if not treated, becomes chronic, and is a persistent source of oral sepsis, the septic products in this case being partly absorbed by the lymphatics and partly swallowed. It is therefore quite clear that the most rational method of eliminating these various sources of sepsis and septic absorption is to take means to prevent the commencement of the carious process at the enamel surface.

\section{Other Sources of Oral Sepsis.}

Any chronic inflammatory lesion within the mouth is to be regarded as a source of oral sepsis; in many of these conditions pus is present from the commencement, and in many others suppuration is sooner or later established.

Such conditions are the various forms of stomatitis, glossitis, and gingivitis; but the most common of all is that known as "pyorrhœa alveolaris,"* and it may not be out of place to refer briefly here to the pathology of this latter lesion. It is essentially a suppurative process occurring in the joint

* A bad term; various others have been suggested. The author believes that "suppurative dental arthritis" is anatomically and pathologically more correct. See his textbook on "Stomatology." 
around a tooth, between it and the jawbone; it may be localized or general, but usually is found associated with groups of several contiguous teeth.

As a general rule, the resistance of the gingival tissues is lowered either by some general constitutional disorder (i.e., chronic nephritis, diabetes, syphilis) or by the local accumulation of salivary calculus around the necks of the teeth. The " gums" become slightly thickened, congested, and dusky in appearance. Infection by oral micro-organisms occurs, and this affects chiefly the dental surface of the gingival fold of mucous membrane, giving rise to ulceration; soon the infection spreads downwards to the periodontal ligament, the upper part of which is destroyed, leaving a small cavity or " pocket," which fills up with pus. The bone of the socket, being thus opened to infection, is next attacked by a rarefying osteitis; thus, inflammation and destruction of the periodontal ligament, and inflammation and destruction of the alveolar bone continue synchronously, until the joint is totally disorganized and the tooth is so loose that it is either accidentally dislocated or is removed surgically. The duration of the disease may vary from a few months to several years, but during the whole of this time pus is continually being formed and discharged into the mouth. When such a "suppurative arthritis" is general, it constitutes a very potent but frequently overlooked, because painless, source of " oral sepsis," and almost invariably originates grave secondary systemic disorders due to chronic toxæmia and sapræmia.

\section{The Bacteriology of the Mouths of Individuals immune to Dental Caries.}

It is essential in considering the preventive treatment of any disease that we should have a clear knowledge as to whether the occurrence of that disease is due to the presence 
of an infection by some particular organism or group of organisms, or whether it is due chiefly to a lowered resistance on the part of the individual to organisms which may be normally present. In other words, in the case of the particular disease we are considering, we should possess some definite data on the difference between the flora of mouths immune to caries and of those which are susceptible.

Our knowledge on this important point has, unfortunately, been somewhat meagre.

Miller was unable to find any marked difference between the character of the organisms in the mouth of an immune and a susceptible individual. Goadby* states that " in making cultivations from the mouths of natives with good dentitions " he has been struck " with the number of putrefactive rather than acid-forming bacteria present." The natives, presumably, were isolated individuals living under civilized conditions in London.

In order to have some more accurate information on the subject, Professor Champtaloup and the author have initiated an investigation into the bacteriology of the mouths of Maori children immune to caries, and living as far from ordinary civilized conditions as possible. $\uparrow$ The Uriwera Reserve was chosen for this purpose, and the majority of the villages there were visited and the children inspected in IgI2.

The Uriwera is an area of about 2,000 square miles of chiefly forest country, in which no white man has a holding, and in which the natives at present live mainly on kumara, riwai, and maize. Wild fruit is plentiful.

* "Mycology of the Mouth," p. 138 .

$\dagger$ This has only been started since the first edition of this book was completed. A grant was made towards the work by the Royal Society of Medicine. For detailed report see Proceedings, Odontological Section, Royal Society of Medicine, r9r3 
Four films from saliva and gingival deposit were made from fifty immune children as follows:

I. From mixed saliva well-triturated in an agate pestle and mortar (stained Gram).

2. From the gingival " deposit," of which there was in all cases extremely little, and which in the younger children was difficult to find. This was gently mixed with 0.9 per cent. sodium citrate on the slide, allowed to dry slowly, and subsequently stained Gram and counter-stained with weak carbol fuschin.

3. An attempt was made to estimate the number of organisms present in the saliva by making films of mixed saliva and citrated formalined blood, the idea being to count the organisms against the red blood cells, and so obtain an estimation of the number of organisms present in any given quantity of the saliva. It was not found practicable to shake the emulsion with sterile beads, but the saliva was well triturated and the mixture with the blood made as efficiently as possible under the circumstances. It was, however, found that, owing to the clumping of the organisms, their regular distribution and the presence of epithelial cells, containing large numbers of organisms, the slides were not of any practical value, and it was not possible to make a reliable count.

4. One film from the saliva was stained by Ziehl-Neelsen for acid-fast bacilli.

The results of examination of the films thus obtained are summarized in the table on p. 30 (abridged from the detailed report).

The outstanding features may be said to be, firstly, the very large number of organisms present, and, secondly, the great similarity to organisms found in European mouths.

The organisms in the table are, of course, classified only morphologically, and, as far as possible, according to their retention of Gram's stain. 
The organism present most frequently and in the greatest numbers was a Gram-negative coccus which resembled the Micrococcus catarrhalis.

Organisms morphologically classified.

Percentage of Films showing Organism.

(I) In Saliva. $\begin{aligned} & \text { (2) In Gingi- } \\ & \text { val Deposit. }\end{aligned}$

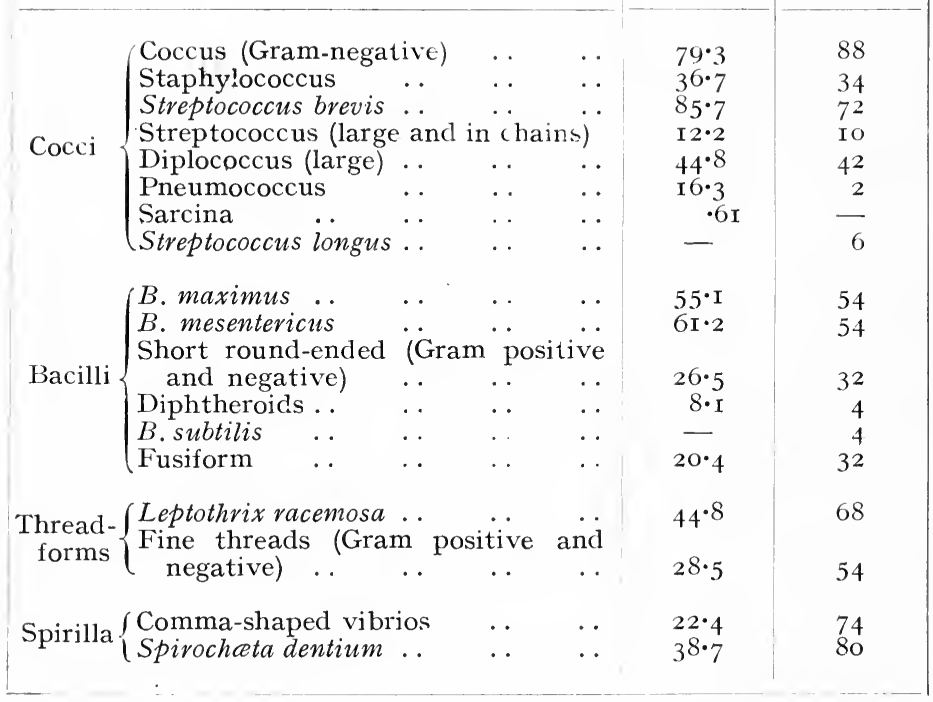

This organism always occurred in clumps, and was present in 88 per cent. of cases in the gingival deposit, and 79.3 per cent. of the salivas. The well-known Streptococcus brevis of the mouth was present in the greater proportion of both salivas and gingival deposits.

The Leptothrix racemosa seemed to flourish luxuriantly in a very large number (68 per cent.) of the mouths, sometimes occurring as isolated specimens, but more often in dense masses. For the most part these organisms were granular in appearance and stained somewhat irregularly. 
In 54 per cent. of gingival deposits and in 28.5 per cent. of salivas there were present also, in large numbers, long, fine, thread-form organisms. For the most part these were Gramnegative, but they appeared to stain irregularly. They might possibly be classed as L. innominata (of Miller).

Spirochætes and vibrios (Gram-negative) were very numerous, and in some cases they seemed to exert an antibiotic effect upon the other organisms, and to have grown largely to the exclusion of the remainder. A fusiform bacillus and Streptococcus longus, and a capsulated diplococcus resembling pneumococcus, were present occasionally; their presence evidently occasioned no reaction on the part of the tissues. A very large streptococcus (probably maximus) was found in a considerable proportion of cases. It occurred in chains of medium length (of from five to ten organisms), and was Gram-positive.

A large, somewhat flattened, Gram-positive diplococcus was similarly present in a considerable number of salivas and gingival deposits. Short, round-ended bacilli, sometimes retaining and sometimes losing Gram's stain, were frequently present. They might have belonged to one of several groups, but it was thought that, for the present purpose, exact identification could not be very accurate and was not specially necessary.

A streptothrix was identified in only one gingival-deposit film, and yeasts similarly.

In one case, in both saliva and gingival deposit, there were present numbers of unbranched thread-forms smaller than $L$. racemosa, with distinct gonidia, the whole retaining Gram's stain very uniformly and densely.

Very large, broad bacilli and diplo-bacilli were found in one or two cases; these were Gram-positive (these might possibly have been a very small yeast form).

Speaking generally, Gram-negative organisms predominated, 
and it seemed that in the salivas there were, perhaps, more organisms present than one would expect to find in a normal healthy European saliva. In the gingival deposits there were on the whole considerably more thread-forms and spirochætes than are usually seen in a similar preparation from the mouths of Europeans (see Fig. 5).

Leucocytes were present in the majority of films from the " materia alba" (Fig. 4), though in most cases they showed plasmolysis, and in others were disintegrating. In only one case was active phagocytosis observed.

It was thought that perhaps the films from the gingival regions would have shown that immunity was due to phagocytosis, but no such evidence was forthcoming.

This absence of active phagocytosis in saliva agrees with what one of us (H. P. P.) found when experimenting on a previous occasion (see page 226).

Desquamated epithelial cells were common in the saliva films, and usually, though not always, these were loaded with organisms-as a rule, the Streptococcus brevis; and it is a question to what extent this may be regarded as a true phagocytosis similar to that which is seen in endothelial cells elsewhere (see page 227).

In the saliva films stained by the Ziehl-Neelsen method, only two acid-fast bacilli were found; these bacilli were stouter than tubercle bacilli, but showed some irregularity in staining. They were rung on the slide and further decolorized with alcohol, when the red colour was discharged, thus showing the bacilli to be of the acid-fast group other than tubercle.

Swabs from the gingival margins were taken in six cases of children immune to caries at the last village visited, and forwarded by post to the laboratory at the University. They occupied, however, exactly a week in transit, so that undoubtedly many of the less hardy varieties died out. 


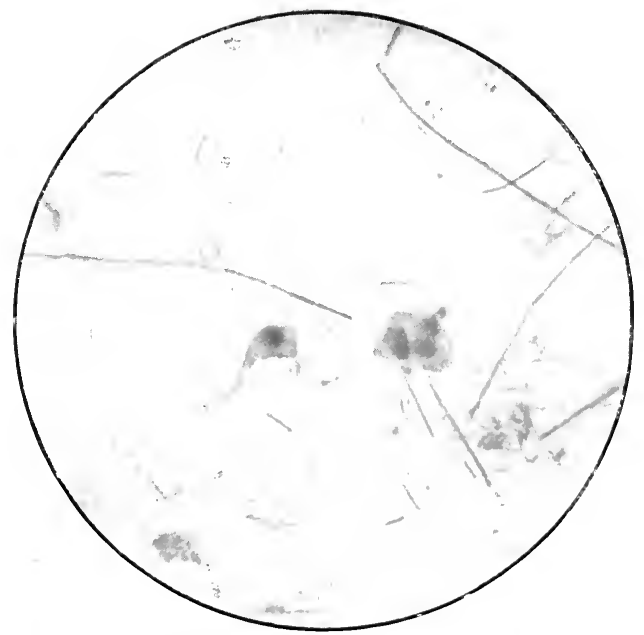

Fig. +.-Film from the Mouth of an Immune MTaori Child, showing 'Thread-Forms and Leucocytes.

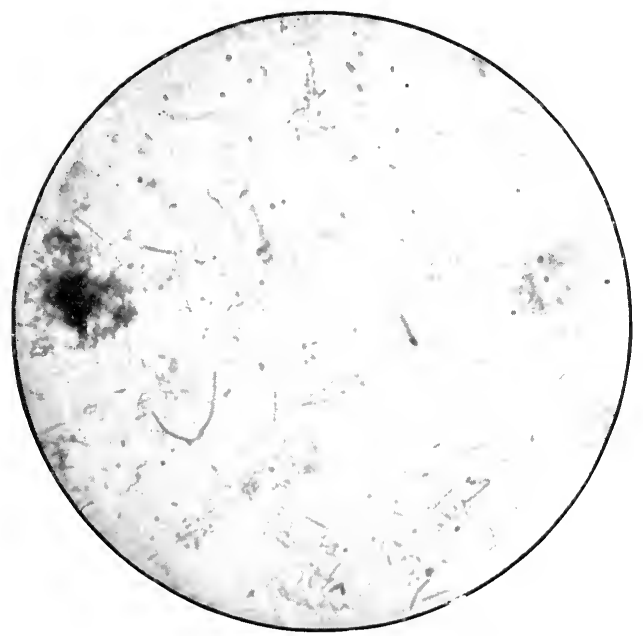

lig. 5. - Film of Organisms made direct from the Mouth of an Immune Maori Child, showing Predominance of Spirochetes. 
The following organismis were isolated from each swab:

I. Pure-culture Staphylococcus aureus.

2. Streptococcus brevis.

Staphylococcus aureus.

Pneumococcus.

Diptheroids.

Large Grani-positive cocci.

3. Streptococcus brevis.

S. longus.

S. bacillus.

Gram-negative bacilli (a few).

4. Staphylococcus aureus.

Diptheroids.

5. Streptococcus longus.

Staphylococcus albus.

Diptheroids.

6. Streptococcus brevis.

A large Gram-positive coccus growing in chains.

S. longus.

Pneumococci.

It is interesting to note two things in connection with the above-firstly, the varieties of organisms isolated by cultivation are for the most part those which were least in evidence in the smears made direct from the mouth, and, secondly, they are precisely similar to what one might expect to find in swabs from individuals in an ordinary civilized community.

We came to the opinion that any conclusions based on cultures made from swabs so long after being taken would be very unreliable, and that if detailed work was to be done it would be necessary to have a bacteriological equipment on the spot-a matter of considerable difficulty and expense owing to the inaccessible nature of the locality. 


\section{Conclusion.}

The result, from morphological evidence, of this partial investigation chiefly goes to show that there exists no great difference between the oral flora of immune and of susceptible individuals, and that freedom from caries in such children is not due to the absence of those organisms which are usually re. garded as causal factors. 
CHAPTER IV

\section{THE ANATOMY AND PHYSIOLOGY OF MASTICATION*}

Ceteris paribus, it is admitted that irregularities in the position of the teeth in the direction of "crowding" lead to an increase in the incidence of caries. This would seem to be obvious from the increased facilities afforded for the lodgment of fermentable foodstuffs, and from the increased difficulty of the teeth being cleaned either naturally or artificially. (Yet this is by no means universally true, as we shall have occasion to point out later. Some mouths with crowded and irregular teeth are comparatively free from caries, and, conversely, many dentures perfectly symmetrical are often rapidly destroyed by disease.)

It therefore becomes necessary to consider briefly the cause and prevention of crowding of the teeth, in order to understand how this cause of the lowered resistance of teeth to caries may be removed. Expressed in a popular way, when the teeth are in marked malocclusion, it is said that " there is not room for them in the jaw," or, put otherwise, that the jaws are too small for the teeth; and this in the majority of cases is a perfectly accurate statement.

In some instances, undoubtedly, the condition arises through heredity pure and simple, a child inheriting the large teeth of one parent with the small jaws and features of the other; but

* Most of this matter is new to this edition. I find upon investigation that the subject is so large (while being quite untouched previously) that only an outline of the subject can be given here. 
in the larger percentage of cases there is no reason to suspect this cause, and the jaws are too small for the teeth, either because they have not developed to their full dimensions, or because they have developed in an abnormal direction.

It may be stated as a physiological axiom, that the bones of those individuals who possess the strongest muscles are much better developed than those of persons with weak muscles. The full development of the bones is dependent upon the musculature, and the latter, of course, upon exercise of function and the amount of resistance to be overcome.

Now, the exercise which the muscles attached to the mandible are called upon to perform is chiefly that of the mastication of food. But mastication is a very varied and complex act; it therefore becomes necessary to consider it in more detail than has hitherto been done.

\section{The Brief Anatomy of the Muscles concerned and the Direction of their Tension.}

The Masseter Muscles arise from the lower borders of the zygomas and converge towards their attachments to the ascending rami of the mandible. In contracting, therefore, they have an expanding influence upon the jaws. These muscles together have, in a normal jaw, an outward pull of 40 millimetres ( $\mathrm{I}_{2}^{\frac{1}{2}}$ inches). (See Fig. 6.)

The Temporal Muscles arise from the sides of the cranium, and (in man) converge to be attached to the coronoid processes; they therefore exert an expanding effect upon the jaws. (In the majority of animals, on the other hand, the base line of origin is narrower than the line of attachment, and therefore the effect of the muscles is a contracting one.) In a normal human skull these two muscles also have an outward pull of 40 millimetres. The masseter and temporal muscles are concerned chiefly in incision and crushing; there- 
fore these two acts have a beneficial expanding effect upon the maxillæ.

The Internal Pterygoid Muscles arise from a narrower base than their attachments to the inner surface of the mandible, and therefore their action is a constricting one. Their inward pull is one of 60 millimetres (Fig. 7). The External Pterygoids have an inward pull of 50 millimetres. Since these muscles are

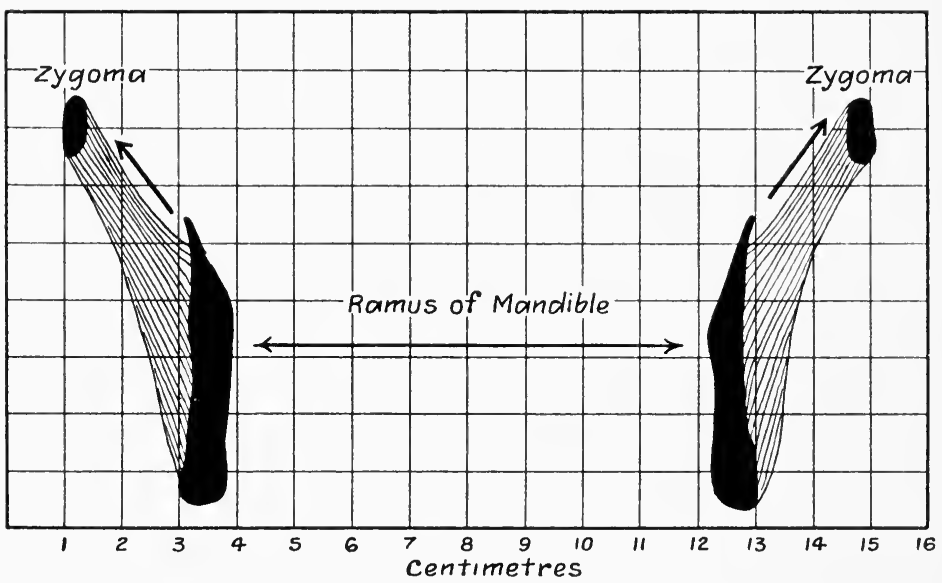

Fig. 6.-Diagram illustrating the Outward Pull of the Masseter Muscles.

The diagram is plotted accurately to scale. Each square= I square centimetre.

largely used in trituration, this latter act can of itself only exert a constricting influence on the jaws.

The Mylo-Hyoid Muscles are attached to the inner surface of the mandible along the mylo-hyoid ridges roughly on a level with the apices of the teeth. They converge inwards and downwards to be inserted in the body of the hyoid bone. The function of the muscles is chiefly developed in swallowing. These muscles represent an inward pull of 32 millimetres. Their action, therefore, is clearly to constrict and narrore the mandible (Fig. 8). 
The Genio-Hyoid and Genio-Hyoglossi Muscles act similarly to the mylo-hyoid, except that they tend to pull the

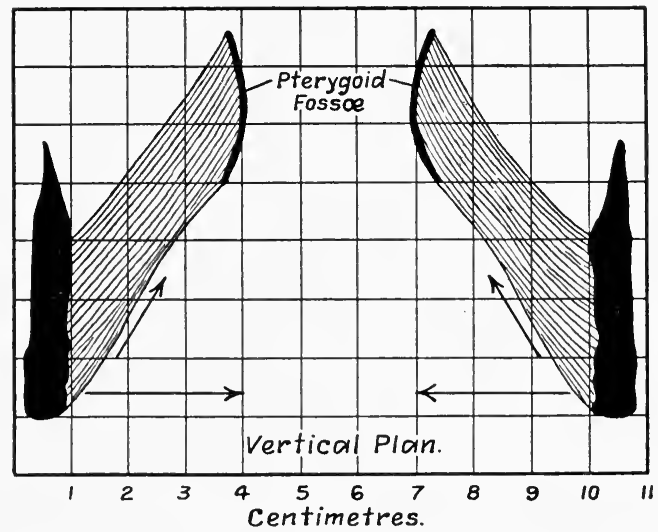

Fig. 7.-Diagram illustrating the Considerable Inward Pull of the Internal Pterygoid Muscles.

Plotted to scale.

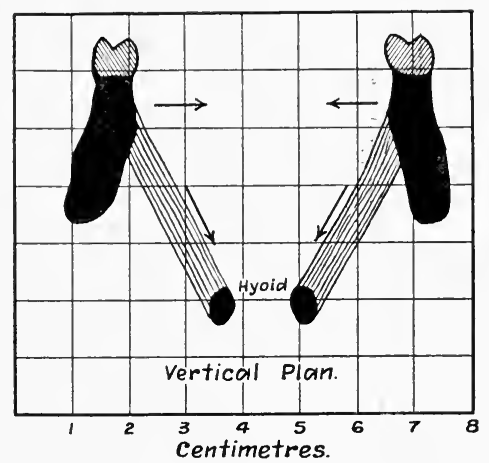

Fig. 8.-Diagram illustrating the Inward Pull of the Mylo-Hyoid Muscles.

Plotted to scale.

mental process of the mandible more directly backward. Their unopposed effect is to produce a chinless mandible. 
These muscles are largely concerned in trituration, sucking, and swallowing.

The Intrinsic Muscles of the Tongue.-Briefly, these are four in number. The superior lingualis, the most superficial muscle, runs in a direction forwards and outwards from the base to the sides and tip of the tongue; its action, therefore, is to shorten and contract the tongue. It could exert no expanding force on the jaws.

The transverse lingualis forms the largest portion of the third layer of muscular fibres of the tongue. It arises from the median septum on each side, and passes outwards to be inserted in the submucous fibrous layer at the side of the tongue; its action, therefore, is to constrict and narrow the tongue.

Intermixed with the latter muscle are two sets of vertical fibres, one derived from the genio-hyoglossus, and the other forming part of the vertical lingualis. When the former of these acts, the tongue is depressed and rendered concave; hence no expanding force on the jaw is developed. The latter muscle, the vertical lingualis, extends from the upper to the lower surface of the tongue, and in the anterior part of the tongue extends to the borders free of the genio-hyoglossus fibres. The action of this muscle is to flatten and broaden the tongue. It is, in fact, the only intrinsic muscle of this organ which is capable of producing any expanding effect on the jaw's or dental arches. Now, this muscle is, I think, but little used in mastication; if at all, it is used only in pressing softer substances against the palate. It is, I believe, chiefly concerned in speech. The other three muscles, on the other hand, which are concerned in mastication, are incapable of exerting any expanding force on the jaws. 


\section{The Physiology of Mastication.}

Mastication as a whole process may be taken to include several distinct and separate stages, each having its own particular function, and each involving the use of a distinct set of muscles. These stages we may enumerate as-(I) Prehension; (2) incision; (3) crushing; (4) trituration; (5) swallowing. It will be necessary to consider each of these in some

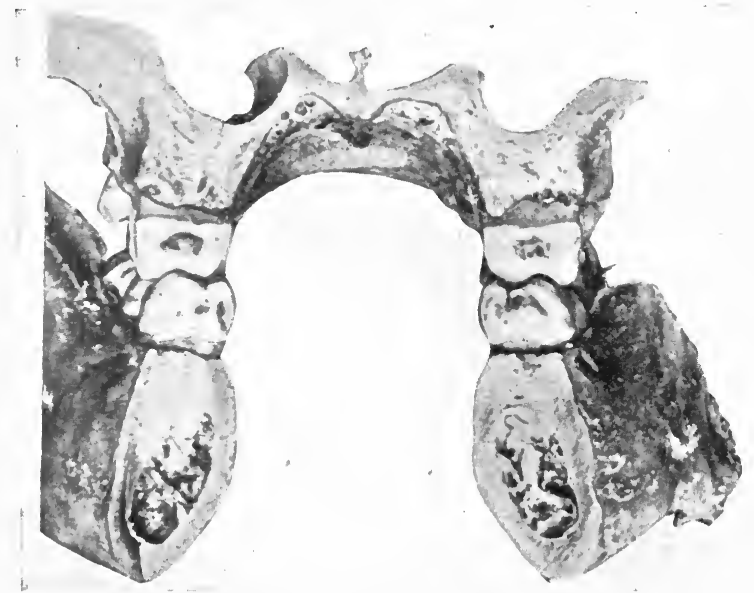

Fig. 9.-Transverse Section of Jaw in Molar Region Showing Interarticular Inclined Planes.

(By permission of Professor Cryer.)

detail, and to follow the effect which the exercise of each is likely to have upon the development of the jaw-bones.

But before doing so, it is necessary to lay down certain axioms upon which the subsequent deductions must be based.

The first, as stated above, is the recognition of the effect of muscular action upon bone- that the full development of bone is dependent upon the functions of the attached muscles, and also that, if a muscle act on a bone in a particular direction unopposed by an equal muscular action in an opposite direction, 
the bone will tend to develop or be curved in the direction of the major muscular tension. (The applicability of this " axiom " will, of course, be modified by the development of organs surrounded by the bone and by the force of gravity.)

The second is that to a large extent the lower jaw is the dominant factor in determining the size and shape of the dental arches, for the following reasons:

(a) To the lower jaw all the most important and strongest muscles involved in the processes of mastication are attached.

(b) The lower teeth articulate within the upper teeth and also in the cheek region by means of double inclined planes, as may be seen in Fig. 9. Thus, provided articulation is normal, any movement inwards or outwards of the active lower jaw must be followed by the passive upper jaw.

(c) Each lower tooth erupts first, and thus to a large extent, under normal conditions, determines the position of the corresponding upper tooth.

Prehension.-This is an act which is little performed at present. It is an act which civilization has almost completely banished; the knife and fork have taken its place. It is only seen when a raw fruit, such as an apple, is eaten in the hand. It is interesting here to recall that, contrary to what may be thought, the universal and common use of the knife and fork at meals is of comparatively recent introduction. The use of knives at mealtimes, of course, is of very ancient date, going back probably to Paleolithic times; but the custom was, right down until the seventeenth century, to use only a knife in a crude way, the meat or other food being held in the fingers and prehended by the incisor teeth, division being accomplished by a combination of pulling and incision. William of Ruysbruck mentions the Tartars as using a "furcicula" in I253; the Spaniards and Italians are cited by Hakluyt as using forks in 1598 ; but they were not introduced into England from the latter countries until the seventeenth century. 
In Prehension and the First Part of Incision the jaw is drawn forwards and the lower incisors braced up edge to edge with the upper incisors by the superficial parts of the masseter muscles, and to a small extent by the temporal muscles (expanding muscles); perhaps also the external pterygoids act, but without much force. Thus the food is gripped. It is then, by the hand in primates, pulled away from the teeth; in lower animals the head as a whole is pulled away from the food, and thus the latter is avulsed or divided. In both cases a forward strain is placed upon the crowns of the incisor teeth, which is only opposed by the portion imbedded in the bone; therefore the teeth come to slope forwards thus $\mathbf{y}$. This prehensile type of teeth and jaws is not common now, but still exists in some of the more primitive races of mankind. Nevertheless, the action is a beneficial one, in that the lower jaw has to function in a forward position, and develops accordingly. It seems very probable that the increasing frequency with which a narrow, distally-occluding mandible is seen nowadays is an expression of this lack of prehension-i.e., lack of masseter action (anterior portion), lack of expansion, and lack of forward thrust.

At the same time, of course, one would not recommend a reversion to forcible prehension. Its gradual elimination has no doubt been largely responsible for the change from a prognathous to an orthognathous type of face. In this, as in all things, what is necessary is the maintenance of just such a balance of function as is required to preserve that anatomical form which we have come to regard as most desirable and "normal."

The Second Part of Incision is a scissors-like action, by which the lower incisors (and the jaw as a whole) are drawn backwards and upwards by the deep part of the masseters and the temporal muscles (expanding effect).

This function, again, is rapidly falling into abeyance, owing 
largely to the universal use of the knife and fork. Children are taught at a very early age to use these articles of elegance as deftly as possible, and to " put only small pieces into the mouth," which obviously means that the food is placed posteriorly to the incisor teeth, and therefore the latter do not function at all. This being so, the orbicularis muscle is not brought into play, and so its moulding influence on the dental arch is lost.

Crushing is performed by the forcible, vertical raising of the lower jaw by the masseter and temporal muscles, with an expanding effect on the jaw.

This is a phase of the masticatory act which is becoming and has become much less common amongst civilized communities. We do not eat many things which require any crushing, such as hard fruit, or hard crisp crusts, nuts, etc., and for a child to crack nuts with the teeth is regarded with horror; but we eat increasingly either much softer things, or, as I believe, on the whole, much tougher.

The heresy that hard things are harmful to the teeth, promulgated alike by dentists and laity, is responsible for a large percentage of the incidence of caries. Children (and adults) are carefully warned not to crack nuts with their teeth, not to bite anything hard, lest they should damage the enamel. The consequence of this is that the majority of people are of the opinion that the "enamel" is little more than a veneer, a delicate tissue easily destroyed, and great care is taken (as they think) to preserve it. Enamel - it cannot be too widely known and appreciated-is the hardest organized tissue in Nature. Its thickness over the cusp of teeth is quite considerable, and such as, under ordinary circumstances, would take very many years to wear through. Its average thickness is 2.3 millimetres, and Black* has shown that the enamel of ordinary teeth will resist without fracture a force of $35^{\circ}$ pounds suddenly applied (hard vulcanized rubber being against the enamel).

* "Operative Dentistry," vol. i., p. I63. 
The eating of hard substances is beneficial, too, to the vitality of the attachments of the teeth to the jaw-bone.

Black has also demonstrated, by means of the gnathodynamometer, that the force of the bite or strength of the masticatory muscles may in favourable individuals be equal to 300 pounds, but that in people whose diet is habitually a soft one the maximum pressure capable of being exerted may be as low as 50 pounds; and, as he points out, once this soft-food habit has been established it tends to become permanent, since the periodontal tissues become tender from lack of pressure and any increase of force is resented by the teeth.

(Bearing in mind the increasing frequency with which this region is so lowered in vitality or resistance as to permit of the entrance of micro-organisms and thus originate chronic suppuration and oral sepsis, the decrease of the crushing function, from this aspect also, becomes very significant.)

Trituration or Grinding.- In this stage the food is placed between the molar teeth on one side of the mouth by the tongue and kept there by the action of the buccinator muscle. The buccinator presses inwards, the tongue presses outwards; therefore they negative each other as regards any expanding effect on the jaws (Fig. I0). It is necessary to observe, also, that in this act of trituration it is the tip of the tongue which, acting like a pseudopodium, performs the function of moving the food about and keeping it in place. The tongue is retracted, narrowed, and twisted upon itself; it is not expanded. The lower jaw is then drawn first outwards and next forcibly upwards and inwards, so that the lower molars grind on the upper molars in a lateral direction-namely, from without inwards. I have never observed, either in animals or man, trituration in the opposite direction.

For these reasons it is clear that the muscles concerned in this movement are the pterygoids acting unilaterally. The resulting effect on the mandible, therefore, is a narrowing, 
constricting one, on account of the direction of tension, as stated above.

I formerly stated* that the food of civilized communities was getting softer and more pap-like. Having further investigated the matter, I desire to considerably qualify these statements. The mistake lies in not discriminating between the terms "tough" and "hard." The significance lies in the fact that tough things require triturating, and therefore ptery-

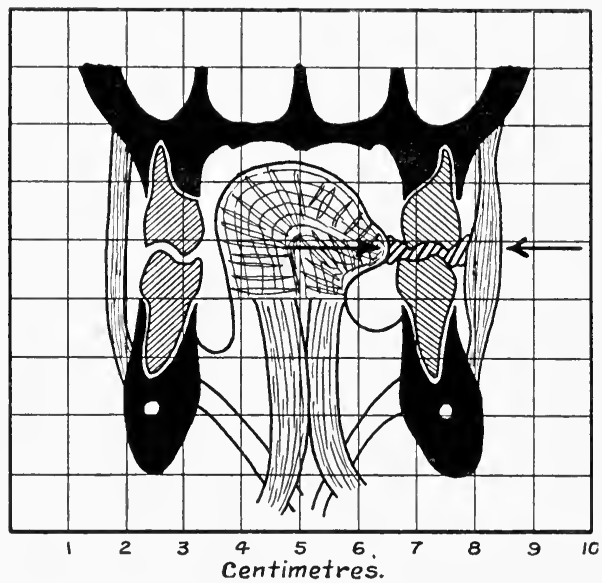

Fig. io.-Diagram Representing the Position of the Tongue in Trituration, and also the Neutralizing Action of the Buccinator and Tongue Muscles.

Plotted to scale.

goid and constricting action; whilst hard things require crusl-ing, and therefore masseter, temporal, and expanding action. Our dietaries are, I believe, much less hard than they used to be, but also I believe they are, on the whole, much more tough. This opinion, which has gradually been forced upon me, is based upon the following facts and observations:

I. All primitive peoples were and are excellent cooks, for * In the first edition of this book. 
the most part steaming nearly all their food until quite tender. Roasting, grilling, stewing, are comparatively modern arts.

2. Thefood was and is always carefully prepared by grinding, washing, or sieving, before cooking.

3. Probably more than a half of the inhabitants of the world at the present time- the southern and eastern Asiatics, the Hindus, and the native races of South Africa-live on what even the most civilized individuals would regard as extremely soft food,- namely, boiled rice, maize, and millet (see Chapter XVIII.). So also the Scotch Highlander's staple food is, or was, porridge, and the Irish peasant's potatoes, neither of which could be regarded as other than soft. Yet all these races have characteristically well-shaped and normally developed jaws and teeth.

4. In conversation with natives-Melanesian and Polynesian-I have found the information always forthcoming unhesitatingly that a white man's meal requires more chewing than their own.

5. The following are quotations from replies to inquiries that I have recently made in Fiji and the New Hebrides groups:

Fiji---The staple foods are vegetables. "Puddings with cocoanut dressing, made by first roasting taro or bread-fruit: and then pounding it into a stiff paste, are practically swallowed without chewing."

" On the whole, a native repast is not masticated so much as an English meal, because there is less to chew."

Mota, New Hebrides. - "Their [the natives] staple food is cooked yam; it is eaten morning and evening-generally roasted, sometimes boiled, often grated and made into a sort of pudding (loko) in the ground oven. Bread-fruit is next in utility, and is either roasted or converted into a dainty mash called 'lot.' Bread-fruit is dried in the off season, when it becomes crisp, like hard biscuit, and is thus eaten (kor) or 
softened in cocoanut sauce, which is a mixture of young cocoanut flesh and sea-water."

"No, I should not think that an islander's ordinary meal would require more mastication than do our meals."

Sir George Simpson, who visited Hawaii in the early part of last century, states that the natives' staple food was poi, a sour kind of porridge, which was bolted in enormous masses without mastication.

Professor Marshall, who has just returned from Tahiti, informs me that the ordinary food of the natives certainly does not require so much mastication as does our " civilized food."

6. From actual subjective experience of living amongst the less civilized Maoris I am able to corroborate all the above statements. Meat, when cooked by Maoris, was always extremely tender, and their meals called for little or no trituration. I examined numbers of children with perfect jaws and teeth, yet these same children lived almost entirely on soft cooked kumara and riwai, and occasionally maize. Not only so, but they actually refused to eat the crust of ordinary white bread when offered to them, saying that it was not soft enough.

7. Very much more meat is being consumed in the present day than ever before by civilized communities-in some cases as much as 2.40 pounds per head per annum; and I venture to assert, from personal experience, that for the most part the meat when cooked will be vastly tougher, and hence require more trituration, than would be the case if cooked by primitive peoples. Again, take another staple article of diet, bread. As it is usually cooked nowadays, the crusts are tough and rubber-like instead of being crisp and hard.

Now, the important point is that tough things, especially if incision (as it is) is avoided, call for a lateral grinding movement of the mandible and a development of the pterygoid muscles. 
This, as has been shown, leads to contraction and not to expansion of the jaws.

Swallowing, although not usually considered as part of the masticatory act, is really its terminal phase, and it is important to consider it in this connection, because certain of the muscles attached to the lower jaw take part in the movement. The muscles are those attached to the inner surface of the horizontal ramus of the mandible and certain of the hyoid muscles.

Swallowing may be abnormal both as regards character and frequency, though this may depend somewhat on what is regarded as the normal.

The act of swallowing is becoming very abnormal, I believe, in both ways.

Primitive and natural races eat far less frequently, and so exercise the swallowing muscles much less than we do at the present time. In many such races only one meal a day is taken, in the majority only two, whereas among hypercivilized people five or six meals a day are becoming quite common. Again, the swallowing of small boluses, such as " manners" now demand, probably requires more contraction of the constricting muscles than do the large boluses of primitive peoples.

Spasmodic swallowing efforts of an abnormal and chronic character are common in children affected with adenoids and posterior rhinitis. In these cases* the child is constantly making strained efforts to swallow either post-nasal growths or the thick viscous secretion on the posterior pharyngeal wall. The superior constrictor, the mylo-hyoid, and the digastric muscles are therefore frequently and forcibly contracting, and these all produce an inward pull upon the mandible (see Fig. I8).

We have, therefore, by a process of analysis, elucidated the

$*$ This point is developed more fully at p. 63 . 
fact that modern dietetic habits are tending to the hypertrophy of those muscles which contract the jaws and to the disuse of those muscles which would expand the jaws.

\section{The Effect of Speech and Emotional Expression.}

The muscles attached to the jaws and used in speech and in the expression of the emotions are chiefly (I) the orbicularis oris and those numerous muscles which radiate out from it

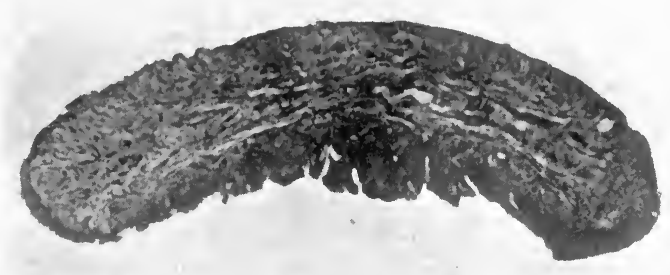

Fig. iI.-Transverse Section through the Anterior Third of an Adult Human Tongue.

to find attachment to the mental, zygomatic, and nasal processes, and (2) the intrinsic and extrinsic muscles of the tongue.

The effect of vigorous development of the first group on the jaws is to produce shortened, well-rounded dental arches, and to develop the mental process or chin, because their origins are posterior to and wider than their points of insertion; the action is somewhat like that of two fans, with contracting ribs, united by an elastic loop. The effect of developing the second group of muscles is to broaden the vault of the palate. This is brought about in the following way. The intrinsic muscle of the tongue chiefly developed is 
the vertical lingualis; this, by contracting, broadens the tongue, and so, when it is forcibly raised between the upper premolar teeth in vigorous speech, the dental arches are widened. The posterior portion of the palatal vault tends to be widened and depressed by the tensor palati muscles, and widened by the levator palati, stylo-glossus, and glosso-pharyngei muscles.

It is interesting and important to notice the difference between a triturating tongue and one which is used for

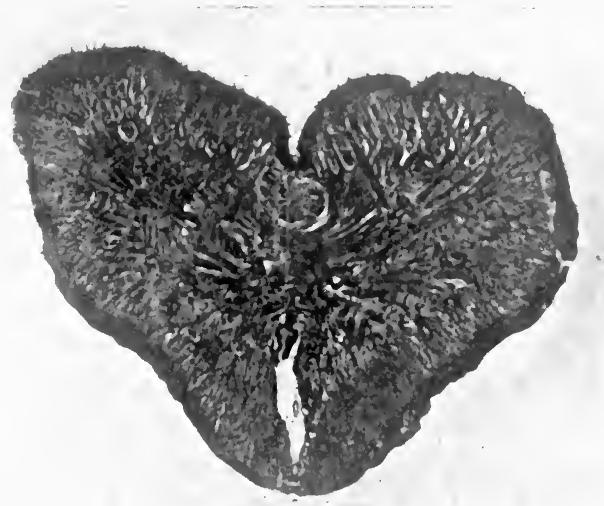

Fig. I 2.-Transverse Section through the Anterior Third of A Sheep's Tongue.

speech ( $c f$. Figs. II and I2). These sections were taken as nearly as possible from corresponding situations in the anterior portion of a sheep's and of a human tongue. An examination of such sections makes it quite obvious that trituration tends to produce long, narrow tongues, and that speech tends to flatten and broaden the tongue.

Speech is a function which, I venture to suggest, is falling into desuetude. We are, comparatively, becoming silent com. munities. Our business, pleasures, and recreations are for the most part undertaken as silently as possible; obviously, 
in adults this would have but small effect upon the jaws. The important part is that it affects also young children. Children are sent to school at increasingly early ages; they are taught to keep silent-that " speech is silvern, silence is golden "; they are taught to speak in modulated tones, to avoid gesticulations and grimaces, and generally to stifle emotions; they sit for long hours with flaccid facial muscles, whilst their visual centres are being increasingly stimulated. $V$ ision (close and strained), on the other hand, is being utilized more and more, and perhaps excessively so. The great majority of adults and children at the present time rely on acute vision almost entirely for both work and recreation. The effect of hyperdeveloping the visual centres in the brain and repressing the speech centres will be, I think, to produce long narrow heads especially since manual dexterity is not increasing but decreasing, and long narrow heads will tend to be associated with narrow jaws.

Compare the above with the picture of " native" children. They are always chattering, shouting, laughing, grimacing. They speak and smile with expression, giving full play to their facial muscles. Their work, pleasures, and recreations are muscular ones; they learn by hearing and not by reading.

\section{Summary.}

The effects of the various functions of the different muscles which we have discussed already, too briefly perhaps, are further summarized in the table on p. 53.

In conclusion, it may be said that the result of modern usage as regards mastication and speech is to produce jaws of a narrow character and a state of unstable equilibrium of the dental arches. Thus, favourable situations for the occurrence of caries are multiplied by the consequent irregular and crowded disposition of the teeth. 


\begin{tabular}{|c|c|c|c|}
\hline \multicolumn{2}{|c|}{ Physiological Function. } & $\begin{array}{c}\text { Effect on Morphology } \\
\text { of Jaws. }\end{array}$ & Occurrence. \\
\hline Incision & . & $\begin{array}{l}\text { Anterior thrust to } \\
\text { mandible. Ex- } \\
\text { pansion }\end{array}$ & Falling into disuse. \\
\hline Crushing & $\cdots$ & $\begin{array}{l}\text { Expansion. Tone to } \\
\text { periodontal liga- } \\
\text { ments }\end{array}$ & Not much practised. \\
\hline Trituratio & 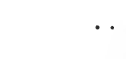 & Constriction & $\begin{array}{l}\text { Increasing in civil- } \\
\text { ized communities. }\end{array}$ \\
\hline Swallowin & & Constriction & Increasing frequency. \\
\hline $\begin{array}{l}\text { Speech ai } \\
\text { sion of }\end{array}$ & $\begin{array}{l}1 \text { expres- } \\
\text { motions }\end{array}$ & $\begin{array}{l}\text { Expansion. Retrac- } \\
\text { tion of incisors }\end{array}$ & \\
\hline Vision (cl & sc) $\quad \ldots$ & $\begin{array}{l}\text { Long narrow heads. } \\
\text { Flaccid jaw and } \\
\text { face muscles }\end{array}$ & Predominates. \\
\hline
\end{tabular}


FACTORS OTHER THAN DIET INFLUENCING THE DISPOSITION OF THE TEETH AND LOWERING THEIR RESISTANCE TO CARIES

\section{A. Habits.}

THE acquisition of certain habits in early childhood tending to constrict the jaws also tends, of course, to diminish the resistance of the teeth to caries. Such habits are usually "sucking habits," such as "dummy" or " comforter " sucking, finger-sucking, and lip-sucking. These all tend to bring abnormal muscular force to bear upon the jaws at improper situations, and so help to distort them and cause crowding of the teeth.

The finger-sucking may as a rule be prevented by tact and firmness (and perhaps a little mustard); the " dummy" sucking, however, is a more difficult question. By "dummy," I mean not only the bottleless rubber teat, but also the teats generally used in the artificial feeding of infants.

There are some things to be said in favour of the rubber teat:

I. They are frequently necessary when natural feeding is impossible.

2. They insure nasal breathing, and in this tend to induce a good habit and one having a beneficial effect upon the shape of the jaws, and must therefore tend to prevent the formation of adenoids.

3. The "bottleless" teat often insures a quiet restfulness 
on the part of the infant-a physiological condition much more conducive to normal growth and development than crying and restlessness.

It has been urged, too, as an argument in favour of the dummy, that since its use is restricted to the first two years of life at most, and since narrowing of the arch rarely appears before the sixth or seventh year, the two cannot possibly be connected as cause and effect. An inquiry into the facts of the case will therefore be beneficial.

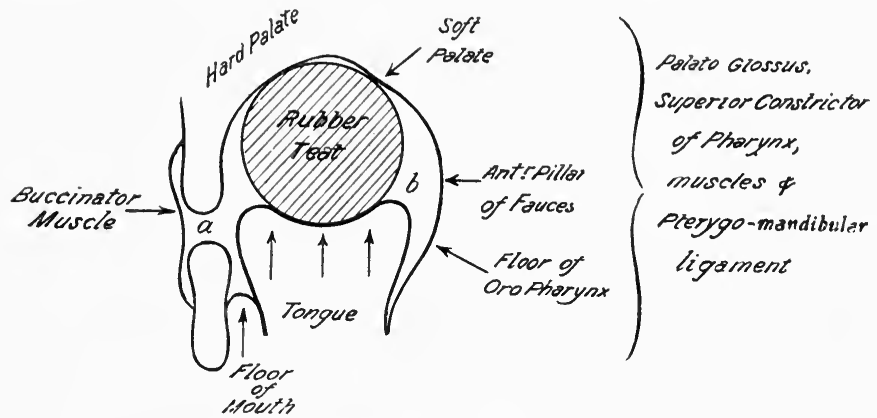

Fig. I3.-Diagrammatic Representation of Oblique Section through Posterior Part of Mouth and Oro-Pharynx, the Left Side being more Anterior than the Right.

The arrows indicate the direction of contraction during "sucking." $a$, Space of negative pressure; $b$, space entirely obliterated during sucking.

The shape of the ordinary teat or dummy is clearly quite wrong. It does not resemble the nipple of a mother's breast in the least, but its chief and most serious defect lies in its abnormal length. The "bulb" of the dummy passes right back to the posterior third of the tongue and the soft palate. The effect of this may be gauged in an adult by sucking the thumb placed as far into the mouth as possible (the shape of the thumb and the length of it correspond proportionately, for the adult, quite closely to that of the dummy for the infant). The thumb is gripped by the tongue, and by the muscles of the soft palate and anterior pillars of the fauces- 
that is to say, by the palato-glossus, superior constrictor of pharynx, and perhaps palato-pharyngeus. These all contract strongly from the periphery towards the centre point of the oro-pharynx. The buccinator muscle is also drawn strongly

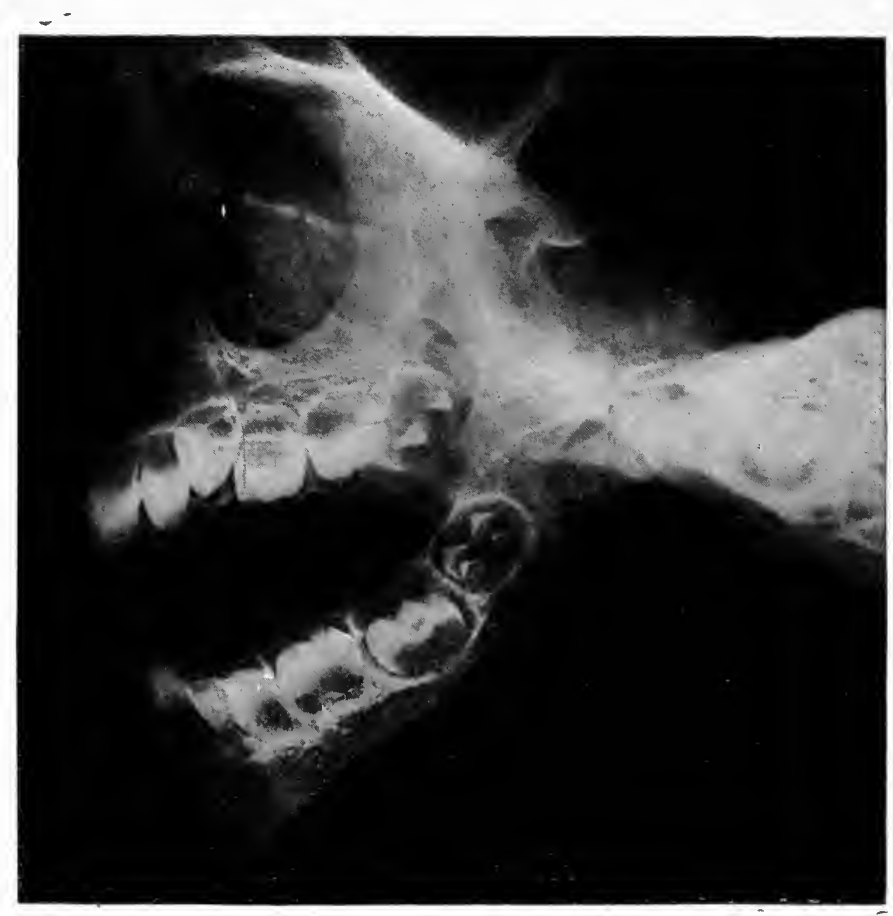

Fig. I4.-Skiagram of the Jaivs of a Child aged Four and a HALF Months.

From Symington and Rankin's "Atlas of Skiagrams," by permission of Messrs. Longmans, Green and Co.)

inwards, and the floor of the mouth upwards (Fig. I3). The pterygo-mandibular ligament, to which is attached the superior constrictor and the buccinator muscles, and which is itself attached to the posterior end of the internal oblique line of the lower jaw, is also drawn inwards and backwards. These 
muscles all act directly or indirectly upon the growing ends of the maxillæ. If a skiagram of the maxillæ at this age be examined (Fig. I4), it will be seen that the terminations of the maxillary bones correspond at this period with the position of the first permanent molars, and it is precisely in this position, when these teeth have erupted, that the narrowing of the arch is most marked.* Other muscles brought into continuous action by such sucking are the genio-glossi and

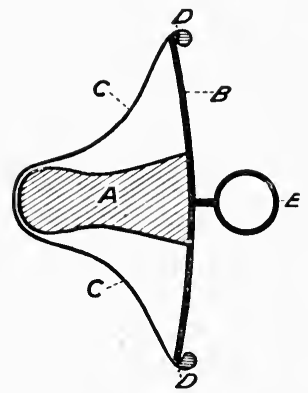

Fig. I5.-Diagram of Natural-Shaped Teat

Designed to imitate the shape and resiliency of the natural breast. It can only be used in the anterior part of the child's mouth.

$A$, Thick" co:e" of soft rubber (solid); $B$, concave disc of hard rubber vulcanized to $A ; C$, large rubbrr teat stretched over $A$ and $B$, and gripping $B$ at $D$ by its thickened edge; $E$, ivo:y or matal ring.

genio-hyoglossi; it thus tends to pull the lower jaw back (or prevent its forward development).

Compare such action with that occurring in sucking a normally-shaped nipple. In the latter case the "teat" does not enter the mouth very far, and the whole action is one of semi-mastication rather than "sucking." The lower jaw is constantly moving, and the proper muscles of mastication are being used and developed.

* Dr. J. Stoddart Barr ("The Effects of Chronic Nasal Obstruction during Childhood upon the Growth of the Teeth and Jaws," British Dental Journal, I9I0, p. 480) has also noted the fact that it is not until the "large permanent teeth" erupt that constriction of the jaws is observed in cases associated with nasal obstruction. 
In natural suckling the action is one of expression by the lower jaw as much as sucking with the mouth. In order that this natural function may be imitated as nearly as possible, I have devised a "dummy" or " comforter" which should have all the advantages, with none of the disadvantages, of the ordinary article. The accompanying diagram (Fig. I5) represents its construction.

The old-fashioned long-teated, long-tubed bottles for the artificial feeding of infants should never be used. It is far better from a dental point of view alone to use no kind of bottle at all, but to encourage and enforce as far as possible the natural suckling of infants. But if this is impossible, then the best kind of bottle to be used is one with a wide neck, like a jam-jar, and having a very large teat resembling in shape the natural breast. One such bottle at least is on the market; even this, however, is not without defect: the "nipple" is far too small. It represents rather the nipple of a functionless, atrophic breast than that of a functioning, secreting breast.

\section{B. The Condition of the Deciduous Teeth.}

Although citing this as a cause of caries may seem like begging the question, yet undoubtedly untreated or maltreated disease of the deciduous teeth tends to increase the susceptibility to caries in the permanent teeth. Caries of the approximal surfaces of the deciduous teeth allows them to come together (see Fig. I6). The cavity rarely remains as a space. The force which brings this about is probably the developing or erupting first and second permanent molars at the back of the jaws. Thus room is found for these teeth at the expense of that portion of the jaw already developed, instead of the jaws developing backwards in order to accommodate them. The result of this is that the permanent premolars, canines, and (often) lateral incisors, are forced to erupt 
in irregular and crowded positions, and the jaws as a whole are smaller than normal. Deciduous teeth, too, may be retained too long or extracted too soon. In either case, irregularities in the permanent teeth are sure to result.

Alveolar abscesses may develop in connection with the temporary teeth, and these frequently cause structural defects

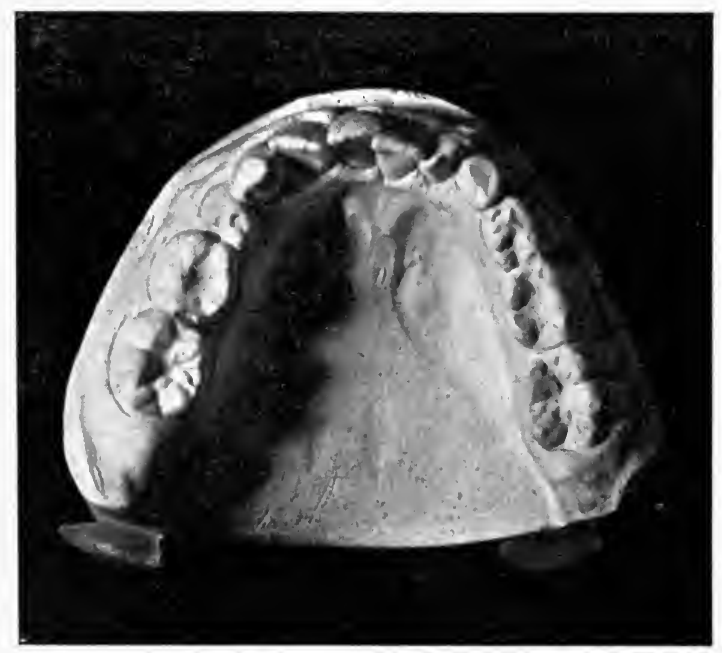

Fig. i6.-Miodel of a Lower Jaw, illustrating the "Loss of Space" Which occurs owing to Caries of the Deciduous TEETH.

Note the manner in which the second deciduous molar on the left has encroached on the space originally occupied by the first deciduous molar.

in the enamel of the permanent teeth, and may thus render them more susceptible to caries. It is therefore of the very highest importance that particular care and attention should be paid to the preservation of the deciduous teeth by the methods which have been already and which are to be hereafter described.

A point which has struck me in the examination of the 
skulls of children of uncivilized races is the marked evidence of much use in the deciduous teeth. Evidently even the very little children used their teeth for their proper purpose, and consequently there are never any signs of irregularities or crowding of the teeth, and there is no trace of caries.

\section{Adenoids.}

The presence of adenoid vegetations in the naso-pharynx has been supposed to lead to a contracted condition of the jaws and to irregularities in the teeth, and hence to favour the development of caries.

Colyer (loc. cit.) has shown that the presence of adenoids in eighty-two cases was associated with an average contraction in the deciduous molar region of $2 \cdot 12$ millimetres, and of I.49 millimetres between the first permanent molars. Such cases showed constantly a crowding of the incisor teeth also.

Mackenzie* also has examined 222 adenoid cases at the Central Throat and Ear Hospital, and finds that of these 43.2 per cent. had normal palates, 39.7 per cent. had " high" palates, and $I 7 \cdot I$ per cent. "Gothic" (high and laterally compressed)-that is to say, 43.2 per cent. of the palates were normal, and $56 \cdot 8$ per cent. more or less contracted.

Thus, there would seem to be an undoubted association between "adenoids" and contracted jaws, but the question which of these is cause and which is effect is not so easy of determination. In the first edition of this book I stated that I was of the opinion that contracted jaws were more frequently the cause than the effect. On further consideration and observation, however, I consider that it is a case of a vicious circle being set up. Either condition may cause the other, but, the circle once established, both conditions tend to become exaggerated.

* British Medical Journal, I906, p. 989. 
We have already referred to the fact that for the normal development of the jaws normal function is (in the absence of a very strong hereditary force) absolutely necessary, and that the avoidance of these normal functions must lead to underdeveloped, narrowed dental arches and jaws. This is not without its effect upon the nasal cavities, as will be seen in Fig. I7.

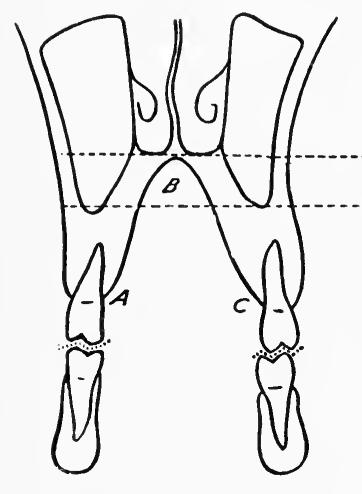

I.

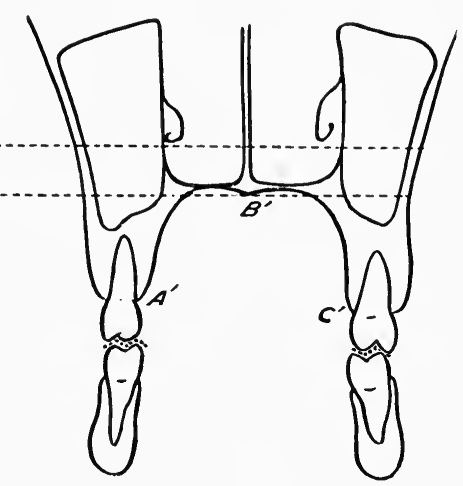

$1 I$.

Fig. I 7.-Diagrams illustrating the Effect of Narrowed Dental Arches upon the Nasal Cavities.

I. represents contracted jaws and potential adenoid nasal fossæ; II. represents the normal. It will be seen that, whilst the distance $A$ to $C$ is much less than $A^{\prime}$ to $C^{\prime}$, the distance $A$ to $B$ is equal to $A^{\prime}$ to $B^{\prime}-i . e$. , the palate has developed upwards at the expense of the nasal cavities.*

The nasal cavities become constricted both in width and depth, and, further, if the nasal septum has an inherent tendency to growth, this can only occur by its becoming deflected. The result is that nasal breathing becomes more difficult, and impossible in any slight inflammation of the

* With this Talbot ("Study in Degenerative Pathology," p. 284) does not agree, stating that the increased height of the palate is more apparent than real. Even, however, if this were granted, it does not affect the point that in these cases there is always a considerable narrowing of the nasal cavities. 
nasal mucous membrane. The patient acquires the habit of mouth-breathing, and adenoids develop in the stagnating nasal cavity.

This appears to me a much more reasonable and logical sequence of events than that the width of the jaws should be influenced by the passive presence of new growths in the nasal cavities.

On the contrary, growths in general cause expansion (or absorption) of bones.

The expanding force of the normal passage of air through the nasal cavities is infinitesimal compared with that exerted normally by the muscles of mastication, and any diminution of the latter force is much more likely to be followed by obvious effect than is total elimination of the former.

Again, it is contended that blocking of the nasal airways causes contraction of the dental arches by promoting mouthbreathing-that is to say, by preventing the upper and lower teeth from coming into contact; but this is the normal position of the jaws and teeth when mastication is not being performed, and therefore cannot be put forward as a cause of the contraction.

Moreover, if the passage of air through the nasal cavities causes expansion, why then should the passage of a similar amount of air through another contiguous cavity, the mouth, produce the opposite effect-namely, contraction of its lateral containing walls ?

The experiments of Ziem and Mayo Collier on this point are, I think, inconclusive, since they blocked one anterior orifice, and thus obviously obtained on that side a narrowed nasal cavity, through producing a negative pressure in that cavity by siphonage posteriorly. But adenoids and tonsils block the posterior nares, and thus siphonage could not occur.

There is, however, another force at work in these cases, which I do not think has been noted previously, and which 
tends to produce narrowing of the mandible and protrusion of the upper incisors.

When children are afflicted with adenoid vegetations and enlarged tonsils they are either constantly making strained efforts to swallow thick viscous mucus, or the growths themselves stimulate the swallowing reflex. The same effect is seen in cases of common colds that are becoming somewhat chronic.

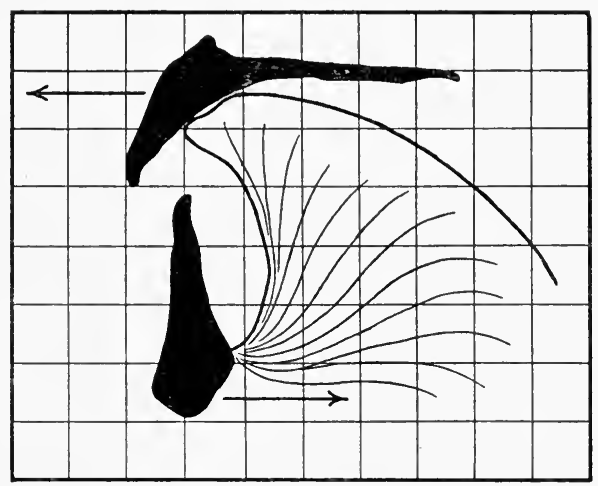

Fig. i8.-Diagram Representing the Action of the Tongue in "Strained Swallowing."

This would promote the occurrence of distally-occluding teeth.

The mylo-hyoid muscles and superior constrictors of the pharynx are constantly and strongly contracting, and since these muscles exert a direct inward pull on the mandible, and this is not counterbalanced by any outward pull of the masseter or temporal muscles, the result is that the mandible is contracted, especially in the region of the molars and premolars. Moreover, during this swallowing effort the tongue gets a purchase behind the upper incisor teeth and exerts a considerable forward pressure in this region-again not counter-balanced by any action of the orbicularis oris. In order to assist in this action the genio-hyoid and digastric 
muscles strongly contract, and thus the mandible and the chin especially are pulled forcibly downwards and backwards (see Fig. I8). A vicious circle is thus originated:

I. A common cold causes blocking of the nasal cavities, swallowing efforts, and contraction of the mandible.

2. The contracted mandible causes contraction of the maxilla.

3. The contracted maxilla causes contraction of the nasal cavities, and their further blocking by vegetation, and this leads to further strained deglutition.

The obvious methods of breaking the circle are, firstly, to treat the conditions surgically, and, secondly, to prevent recurrence by developing the crushing, and therefore expanding, muscles of the jaw much more than the muscles of deglutition.

I do not pretend to think that narrowed jaws dependent on deficient mastication are the sole cause of adenoids; but when the two are associated, I do think that the adenoids are to a large extent secondary to the condition of the maxillary bones. There is no doubt, however, that mouth-breathing is deleterious to the teeth, and favours the occurrence of caries. It tends to keep the mouth dry and negative the beneficial effect of the circulation of the saliva; and it also must promote a constant and enormous increase in the bacterial flora of the mouth by the inhalation of dust-laden air, which always contains numbers of fermentative bacteria - the exact varieties that are principally concerned in the early stages of caries. It is therefore obvious that adenoids may be a factor in the production of carious teeth, and should therefore be eliminated, by hygienic and educational means preferably, as early in the child's life as possible. 


\section{Heredity and Dental Caries.}

There must be in all growth and development two fundamental forces at work-firstly, that mysterious force which we know as heredity, because of which offspring tend to resemble their parents even in minute details; and, secondly, the resultant of all those external forces which are continuously acting upon an organism, and known as environment.

Now, these two forces may act in unison, may both tend in the same direction, or they may be forces acting in different directions, and thus oppose each other. If the first condition obtains, then the hereditary characteristics tend to be perpetuated, and may become more marked. If, however, the second condition be present, a number of variations is possible.

Logically, of course, if the two forces of heredity and environment are equal and opposite, the organism ceases to exist, and for the present argument this variation does not concern us. But if the forces of environment oppose the hereditary tendency to a lesser extent, then more or less modification of the hereditary morphology and structure of the organism will be brought about. And it is largely a question of the relative strength of these two forces that determines whether an offspring which exhibited the normal characteristics of its race strongly in infancy will continue to do so during growth and development towards adult life, or will during this period, when environmental forces are continuously becoming more strong, show modifications of, or departures from, the original characteristics. These modifications may be brought about either by an increase of the environmental forces or by a decrease in " hereditary force," or by a combination of both.

Thus the tendency of the force of heredity is to produce jaws well shaped in paraboloid curves, teeth of a certain size and shape, and regularly arranged in the jaws. But, as we 
have seen, habit and environment (more particularly expressed in the dietary of the individual) may profoundly modify the shape and size of the jaws. In very early childhood, during the period of deciduous teeth, the forces governing the growth and development of the jaws are largely hereditary, and consequently the jaws are usually well formed and the teeth regular in arrangement.

During this period the crowns of the permanent teeth are being developed, and hence heredity commences (at least) to form teeth of the normal size.

The two forces of heredity and environment then come into opposition. Heredity demands a full-sized tooth; the forces of environment, acting on the jaws, limit the available space. The result is a compromise. The jaws develop a little in an attempt to make room for the teeth, and, as will be shown, the teeth are restricted in development.

An instance of heredity and environment acting in unison is seen in the dentition of uncivilized races. Here both forces tend to produce well-shaped jaws and teeth. Instances of environment proving too strong for heredity are only too common elsewhere, but an interesting instance of heredity overcoming environment is afforded by the following case, like which there are doubtless many more:

An Englishwoman had two husbands, the first an Englishman, and the second a negro. The children of the first marriage had the usual jaws and the teeth of civilized races; the children of the second marriage were brought up with the other children, with identical environments, yet they possessed well-shaped jaws and sound teeth.

This influence of a slight balance of hereditary force over environmental forces has been thrust upon my attention during the last few years when I have had occasion to examine the teeth of a large number of "orphanage" childrenchildren in whom the force of environment has been identical 
for all practical purposes, and yet whose jaws and teeth showed wide variations as to shape, disposition, and incidence of caries. This is only to be accounted for by the preponderance in some cases of hereditary force over environment.

Further, there would seem to be every reason for believing that the loss of " hereditary force" tends to be transmitted, particularly when the forces of environment are not acting in conjunction with it. Or, expressed otherwise, the possession of strong, well-formed jaws and regular teeth ceases to be of paramount advantage to the individual in the mastication of food (thanks to the services of the dental surgeon and to the ready acquisition of soft "supernutritious" food), and so, like all other organs or organisms in Nature which have become more or less functionless, they tend to be reproduced in offspring in a correspondingly degenerate condition. Thus, in any particular case we may have a "negative" environmental force combined with a "negative" hereditary force, producing an exaggerated smallness of the jaws and crowding of the teeth-a condition difficult of immediate prophylactic treatment.

The application of an environmental force may possibly accomplish much in early life, but would be more certain of attaining a normal result if applied for one or two generations. Such a condition could, doubtless, be combated by the application of sexual selection, but in civilized communities of the human race parents are not selected on account of their physical characteristics, but increasingly for economic and social reasons alone.

The resultant of the same two "forces" is, of course, expressed in a similar manner in the growth and development of the soft tissues-i.e., in the muscles of mastication and the salivary glands. The effect of the former upon the jaws and teeth has already been noted, and the effect of variations in the latter upon the teeth will be considered subsequently. 
THE FORCES OF NATURAL PROTECTION

I. PASSIVE-THE ENAMEL SURFACE 
THE ENAMEL SURFACE: ITS PHYSICAL PROPERTIES AND POWERS OF RESISTANCE

MucH has been written and much valuable work accomplished on the structure of the enamel of teeth, but all histologists and authors seem to have confined their attention to the minute structure of the body of enamel.

Even Miller, ${ }^{1}$ in his researches on the pathology of caries, seems to have omitted the surface structure of enamel.

Leon Williams, ${ }^{2}$ whose name is inseparably connected with the development and structure of enamel, touched on the subject but briefly when demonstrating the presence of a film on the surface of enamel, especially on carious enamel.

Tomes $^{3}$ refers to the subject only in the following words: "The cap of enamel on a human tooth is of varying thickness, being thicker in the neighbourhood of the cusps than elsewhere. Its outer surface is often finely striated, the striæ being transverse to the long axis of the crown. In addition to this fine striation, there may be a few deeper and more pronounced grooves or pits, which are pathological, and are marks of checks in development more or less complete."

Hopewell Smith ${ }^{4}$ states briefly: "Surface smooth, shiny, sometimes traversed by tiny vertical or horizontal depressions, occasionally scrobiculated, and normally deeply fissured in premolars and molars, and pure white in colour. When fractured, pure white, non-lustrous." 
That the minute structure and physical characteristics of the enamel surface in normal and abnormal conditions are worthy of consideration must be obvious when it is remembered what an important rôle these must play in the question of immunity or susceptibility to dental caries.

It is at the enamel surface alone that the process of caries is able to be prevented or checked, either naturally or by artificial means. This point being passed, the total destruction of the tooth is merely a matter of time (except in those comparatively rare cases of " arrested caries ").

An investigation of the surface of the enamel of a large number of teeth has therefore been made, some of the results of which it is proposed to deal with here.

Over $1 ; 000$ teeth of civilized and of savage races have been minutely examined microscopically and physically.

For the purpose of this investigation teeth were divided into three empirical or clinical groups:

I. Native teeth, comprising those of uncivilized races afflicted but little, if at all, with caries.

2. Sclerotic teeth, comprising that clinically well-known class of teeth which are characterized by their " hardness," usually yellow colour, and immunity to caries.

3. Malacotic teeth (Gr. $\mu \alpha \lambda \alpha \kappa o ́ s=$ soft), also a well-recognized clinical variety characterized by their comparative "softness," whiteness, and susceptibility to caries.

As stated above, these divisions were accepted empirically, though up to the present there has been no scientific basis for such a classification. In fact, the experiments of Tomes ${ }^{5}$ and Black $^{6}$ on the composition and physical characteristics of enamel go rather to negative any such classification, seeing that they agreed in finding no appreciable difference of density, structure, or composition, between "soft " and " hard " teeth, or, at least, not such as would lead to their varying predisposition to caries. 
These views are endorsed by Leon Williams ${ }^{7}$ and by Kirk. ${ }^{8}$ Nevertheless it is a convenient clinical classification, and one which the writer believes is justified by the results hereafter to be described.

\section{Histological Examination of the Enamel Surface.}

In this branch of the inquiry, the first undertaking was a thorough examination of the enamel surface of a large number of all classes of teeth under the microscope by reflected light.

The difficulties of lighting and staining were at first considerable, and, after trying and abandoning many other methods, the following simple method of staining was adopted, as giving the most satisfactory results:

The tooth to be examined is first washed in water and dried, and then the surface well cleaned with xylol or ether and alcohol. The stain used is graphite. This is applied dry on a soft pad (or the finger), and excess removed with a piece of soft smooth rubber. The result of this treatment is that with moderate pressure the graphite is rubbed into all depressions in the enamel surface, whilst it is removed from all elevations; consequently, depressions are stained black, and elevations remain white. Whereas with very light staining, in some cases, the elevations are stained, and the depressions remain their normal colour.

By this method and by the correct interpretation of what is seen a great deal may be learned of the minute structure of the enamel surface.

From such an examination, it is concluded that the surface of enamel is not normally smooth in human teeth nor in many animals. It is, in its normal condition, traversed on its vertical aspects by minute ridges separated from each other by corresponding "furrows," which run parallel to each other in a direction at right angles to the long axis of the tooth. 
These horizontal lines are in some cases large enough to be visible to the naked eye, and must have been noticed occasionally by many who, like myself, thought at first that they were either caused by attrition or were a microscopic form of hypoplasia; yet in all the teeth examined only one failed to show any sign of these ridges and furrows. And it is significant that this exception was an abnormal tooth-a lower molar with a crown elongated antero-posteriorly to twice its normal length, and with an exceedingly rounded, stunted, and single root.

Such horizontal lines (or ridges and furrows), for reasons subsequently to be described, I have termed "imbrication lines."

I have examined microscopically the teeth in every skull in the Anthropological Museum of this University. This collection of skulls is of various races all more or less uncivilized, including Fijians, Australian aborigines, Maori, Moriori, Rarotongans, inhabitants of New Guinea and New Hebrides, Negro, Hindoo, Chinese, Mexican, and Anglo-Saxon. These also, with only one exception, all showed evidences of minute imbrication lines on the surface of the enamel.

In all teeth the ridges are widest and the furrows deepest at a point two-thirds of the distance between the neck and the occlusal surface, or cutting edge. From this point they gradually diminish in size upwards and downwards. At the neck of the tooth they are much more numerous in a given space, but are very much finer and less marked. They extend towards the occlusal surface in an unworn tooth, and do not here diminish so much in size, but they do very greatly in frequency. They also naturally rapidly disappear from this situation when the tooth has been subject to mastication.

Fig. I9 shows the appearance of the "normal" enamel surface of a malacotic tooth at a point two-thirds of the distance between the neck and the occlusal surface (treated by the graphite method and viewed by reflected light under the 


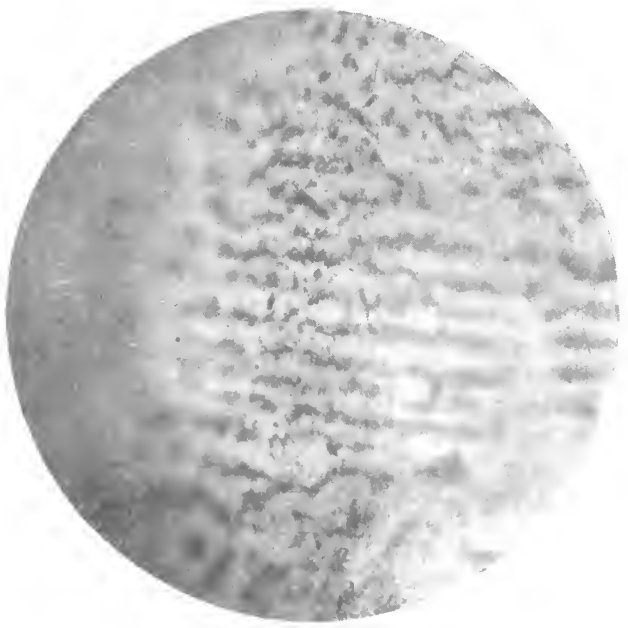

Fig. I9.-Imbrication Lines on a Malacotic Tooth, from the Distal Surface near the "Point of Contact."

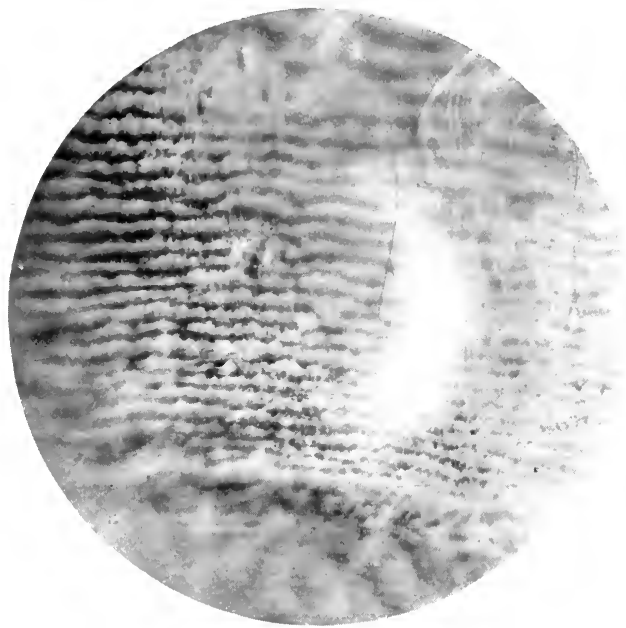

Fig. 20.-Imbrication Lines on a Malacotic Tooth, near the Cervical Region. 
microscope). Fig. 20 is a photomicrograph from the same tooth at a point close to the neck. Fig. 2I shows the character of the imbrication "lines" in a Maori tooth. The average distance apart of these lines was, on measurement with the micrometer, found to be o.or millimetre in the cervical region, and $O \cdot I$ millimetre at a point half of the distance between the neck and occlusal surface.

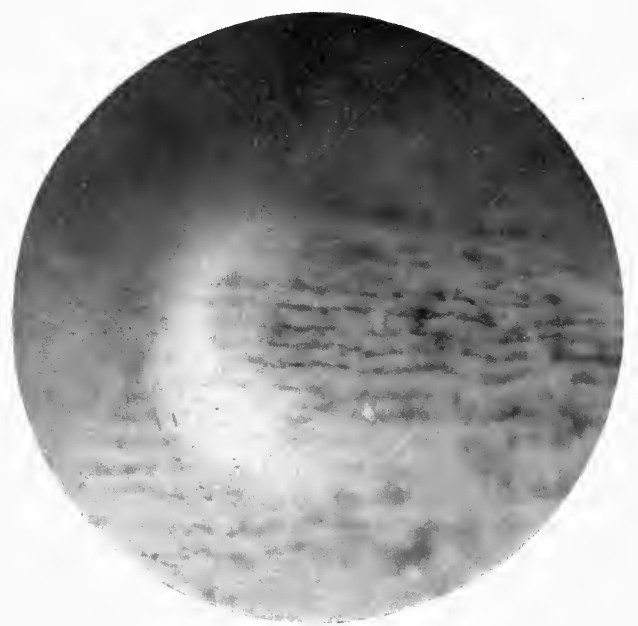

Fig. 21.-Imbrication Lines from the Distal Surface of a MaOri Molar.

With regard to the accompanying photomicrographs, it should be pointed out that the surface to be dealt with is both irregular and convex, and that it is therefore impossible for the whole of the field to be in focus at one and the same time.

\section{The Enamel Surface in Outline.}

Sections of enamel from teeth with the lines well marked show with accurate focussing the ridges and furrows in outline. Fig. 23, which is the same magnification as Fig. I9, and 
viewed with reflected light, shows this. Fig. 24, which is taken from the same section, but viewed by transmitted light, shows this structure even more markedly. The fact that the "outline" of the surface lines is not easy to demonstrate by transmitted light.is due to the thickness of sections of enamel

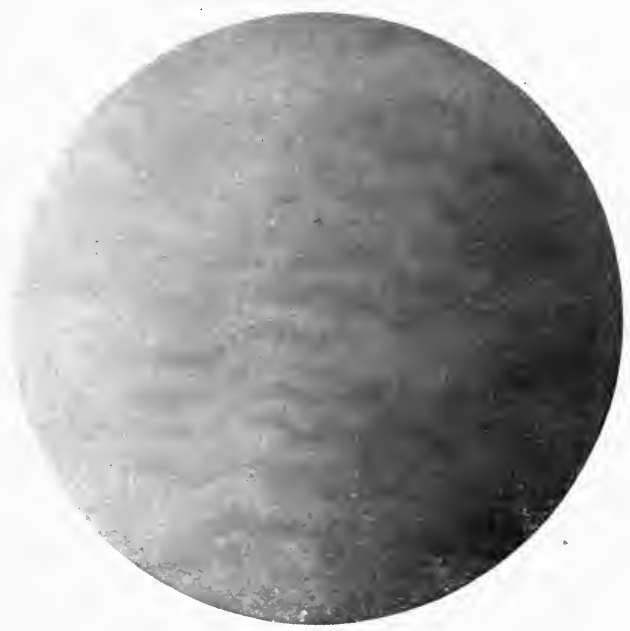

Fig. 22.-Unstained"Strie on the Enamel Surface.

These are not "imbrication" lines, and are possibly the outcrop of Schreger's lines. They can only be seen on highly polished surfaces and facets. (Compare with Fig. 23.)

interfering with accurate focussing;* whereas by examining a section of any thickness by reflected light, only one surface is illuminated, and thus all is accurately focussed.

\section{Curvature of the Enamel Surface.}

In examining the teeth of native races, the enamel surface has the appearance of being more curved than that on the

* The lower edge of the section usually throws a shadow which obscures the detail of the upper edge, and the substage lighting requires adjusting to avord this. 


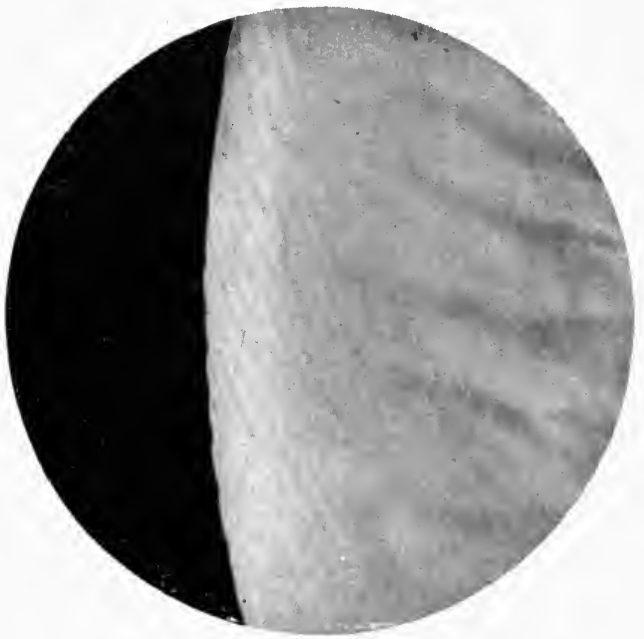

Fig. 23.-Imbrications in Outline.

Note the relationship of the striæ in the enamel to the imbrications (low power, reflected light).

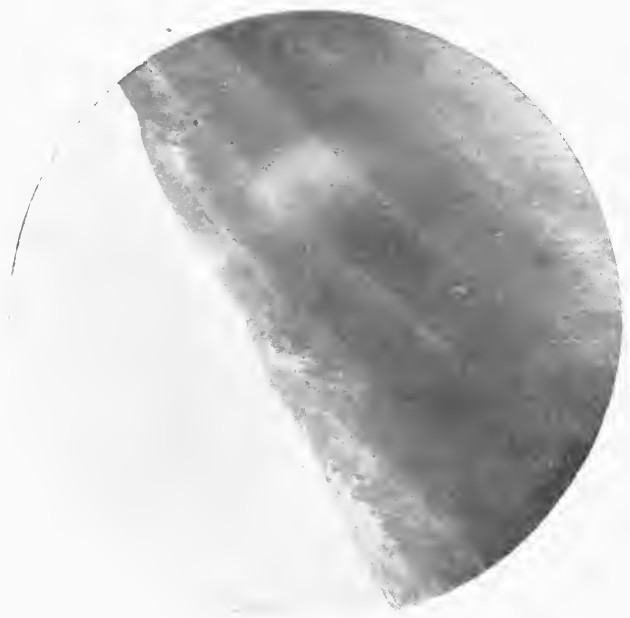

Fig. 24.-Imbrications in Outline

(High power, transmitted light.) 
teeth of modern civilized races; also it seems to arise more abruptly at the cervix in the former class of tooth, resembling the abrupt ridge on the teeth of some carnivora and on human deciduous teeth.

These conditions, although not constant, were sufficiently frequent to suggest the cutting of sections to compare the curvatures and their causes. As a result the causes of these

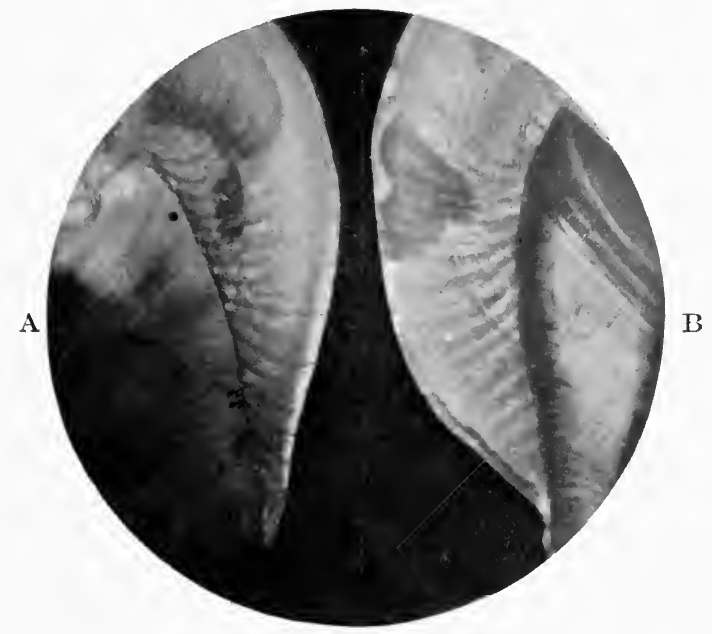

Fig. 25.- $A$, Vertical Section of Enamel of European Molar Tooth; $B$, Vertical Section of Enamel of Maori Molar Тоотн.

conditions were found to be totally different. This will be seen on reference to Figs. 25, 26, and 27.

In the permanent European tooth it will be seen that the enamel rises slowly and gradually from the cervix, whereas in the "native" tooth (Fig. 25) it rises much more abruptly; and this is occasioned solely by the increased thickness of the enamel.

Fig. 26 represents a permanent and a deciduous tooth mounted side by side. It will be seen that there is an abrupt 


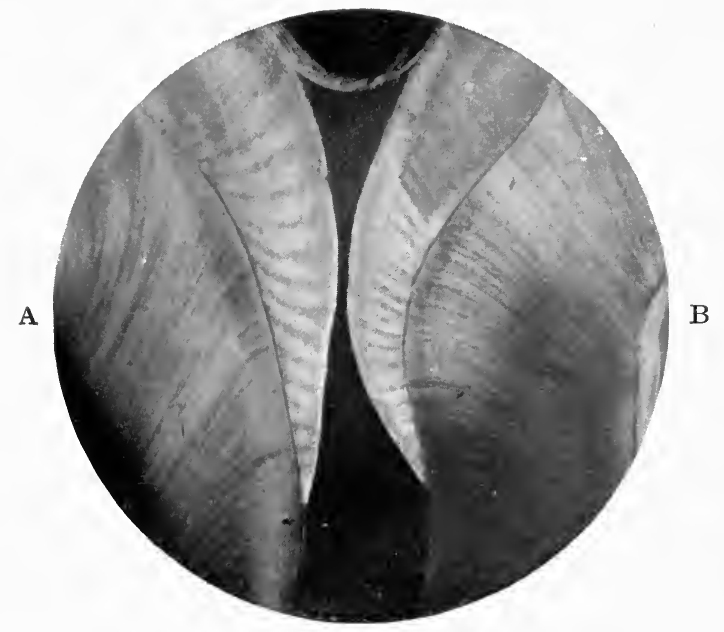

Fig. 26.- $A$, Vertical Section of Enamel of European Permanent Molar; $B$, Vertical Section of Enamel of European DECIDUOUS Molar.

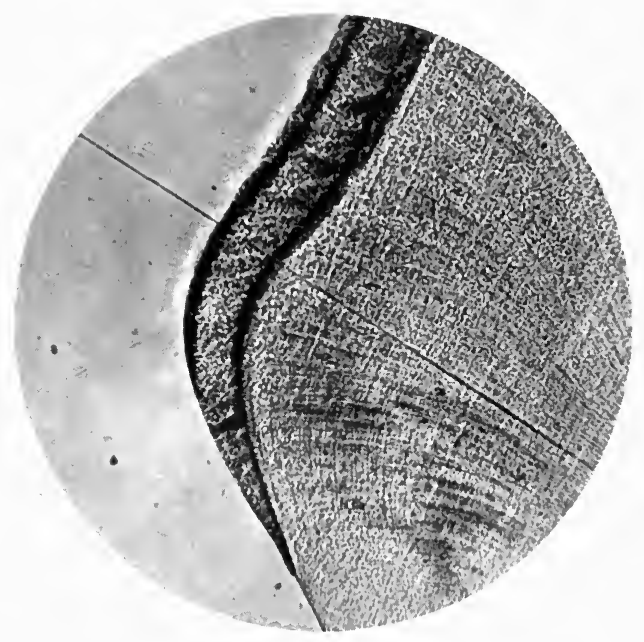

Fig. 27.-Vertical Section of the Molar Tooth of a Dog. 
rise of enamel from the cervix of the milk-tooth similar to that in the native tooth, but that it is caused entirely by an increased thickness of the dentine, as if the dentine germ had bulged out at that point during development, and the normal thickness of enamel formed over it.

Fig. 27 is a section of the molar of a dog, and shows a similar conformation to that of the human milk-tooth.

The effect of this difference in curvature and thickness of enamel in the cervical region is important, in that it obviously, in the native tooth, diminishes the amount of interdental space, and therefore diminishes also the amount of carbohydrate which might lodge there. In the European teeth the space is greater and the enamel is more imbricated, and so food would lodge in greater quantity and would also be more difficult to remove by natural means. It will be shown later that this diminished curvature and increased imbrication are probably intimately connected and arise from a common cause, and that the remedy lies in eliminating this common cause.

\section{REFERENCES.}

1 Miller: "Micro-Organisms of Human Mouth," Dental Cosmos, $1904-05$.

2 Williams, Leon: Dental Cosmos, i 898.

3 Tomes, C. S.: Dental Anatomy, p. 20.

4 Smith, Hopewell: Histology and Pathohistology of the Teeth. p. 20 .

\footnotetext{
5 Tomes, C. S.: Journal of Physiology, r 896, No. 3.

6 BIACK: Dental Cosmos, I895, p. 4 I 7 .

7 Williams, J. L.: Dental Cosmos, i 898 .

8 Kirk, E. C.: Dental Cosmos, I903.
} 


\section{THE ENAMEL SURFACE: THE ODONTOGRAPH}

\section{Graphic Representation of the Enamel Surface.}

THE methods hitherto described of examining and recording the variations in the enamel surface and in the thickness of the enamel require the expenditure of a considerable amount of time upon each tooth; more especially is this so when sections of the enamel have to be cut and examined by transmitted light. It therefore seemed necessary that, if a large number of teeth were to be examined and compared, some rapid and accurate method for the purpose, and for the purpose of mechanically recording the variations, was absolutely necessary.

To this end an assemblage and modification of apparatus used in experimental physiology was made, and the specialized form which it took may be called an "odontograph."

The apparatus may be best explained by reference to the accompanying diagram (Fig. 28).

$A$ is the usual revolving drum used in experinental physiology (the surface of the cylinder being covered with smoked paper).

$B$ is the usual form of stand, with a revolving upright and a movable platform $C$. To this platform is hinged very delicately a long and very light wooden lever, $D$; as a rule this was at least 2 feet in length.

At $E$ on the lever is fixed a needle, the point of which impinges upon the surface of the tooth to be examined. The tooth is fixed by wax to a small carriage, $F$, on four wheels, which runs between rails on the platform. 
The carriage is drawn forwards by means of a string attached to its front part, and passing under the pulley $G$ and over $H$ on to a large receiving pulley, $K$, which is fixed to the revolving axle of the cylinder. Thus, when the drum revolves, the tooth is drawn forward mechanically, and its rate of movement always bears the same ratio to the rate of movement of

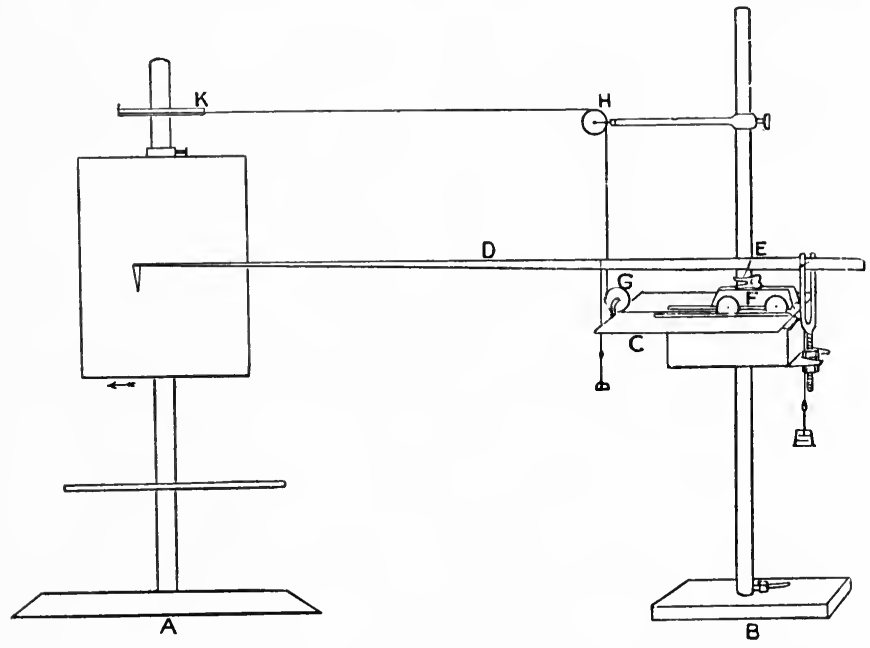

Fig. 28.-A pparatus arranged For recording Odontograms.

the drum. By having several receiving pulleys of various sizes, the rate at which the tooth passes under the needle can be varied as desired.

To the back of the carriage is suspended a weight, in order that the string in front may always be taut. It is also necessary, usually, to suspend a small weight from the lever in front of the needle, in order to avoid instrumental vibration from being recorded.

In this manner, when the drum is revolved, the tooth in passing under the needle raises the latter or allows it to fall, to a very small extent, according as there are elevations or de- 
pressions on the surface of the enamel. Owing to the length of the lever these oscillations are greatly magnified; they are recorded on the smoked paper as curves and waves. Such a record may be most appropriately termed an " odontogram." An odontogram records chiefly two things:

I. The presence or absence of imbrication lines, and the extent of their development.

2. The thickness of the enamel.

The former is obvious; the latter, perhaps, may not be, and will be explained shortly.

Figs. 29 and 30 are the odontograms of perfectly sound and
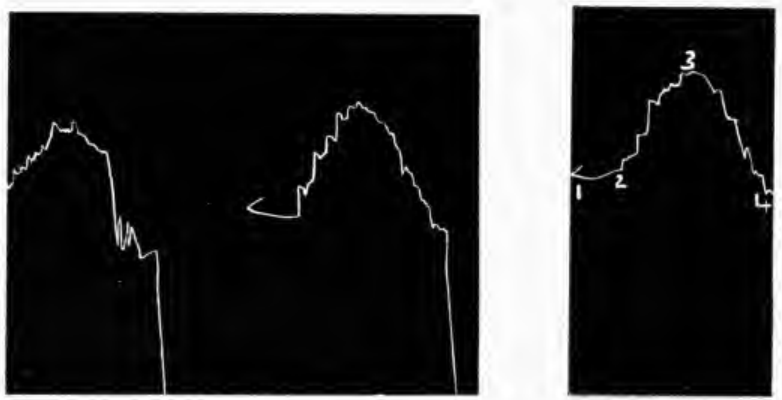

Figs 29 and 30.-Odontograms of Malacotic Teeth.

typically malacotic teeth with well-marked imbrication lines. From I to 2 represent the cement, 2 is the neck, 2 to 3 is the surface of the enamel up to the bulb of the crown, and 3 to 4 from the bulb to the occlusal surface. The secondary waves represent the imbrication lines, and it will be noticed that they are most marked in the first half of the primary curve. The high peak of some of the secondary waves is probably due to instrumental vibration-just as in a sphygmogram-and the needle has therefore at this point failed to record several imbrications.

The odontograms of typical Maori teeth are shown in 
Fig. 3I. It will be noticed that they differ markedly from those of the malacotic teeth. In the first place, the shape of the primary curve is quite different, and, secondly, the frequency and extent of the secondary curves are much diminished. This latter means, of course, that the imbrication lines are much less marked on the enamel surface, but that they are present.

The difference in the primary curve is caused by the difference in the curvature of the enamel surface. The most
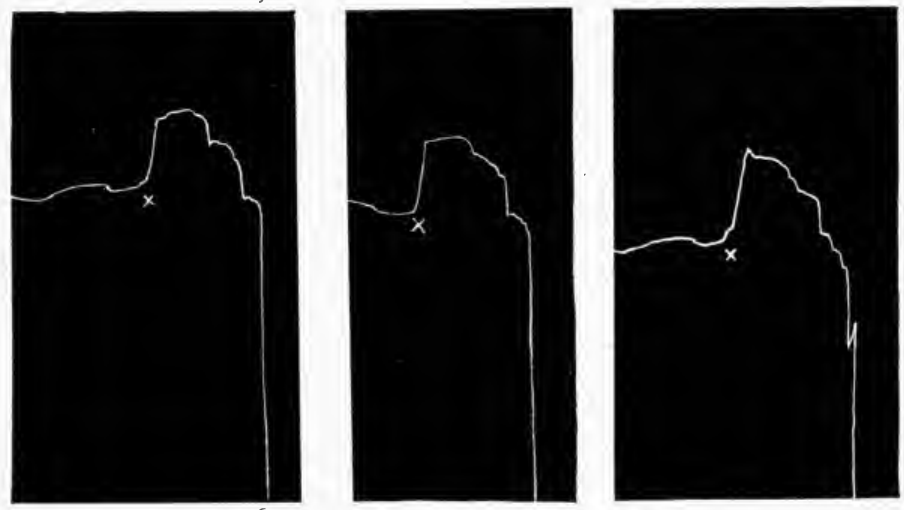

Fig. 3i.-Odontograms of Maori Teeth.

$x=$ commencement of enamel.

obvious difference is the very marked and abrupt rise at the commencement of the enamel; it is this abruptness of rise in this position which is a measure of the thickness of the enamel on the side of the tooth (but especially on the mesial and distal surfaces). This applies only to human permanent teeth; a similar abrupt rise is noticed in the odontograms of human deciduous teeth (Fig. 32) and of the teeth of carnivora, but, as we have already seen (Figs. 26 and 27), it is due in these cases to a bulging out of the dentine. The actual height of the top of the curve bears no relationship to the thickness 
of enamel, since this can be artificially altered by changing the position of the tooth on the carriage; but if a vertical line be dropped to the base line from the highest point of the curve, and the distance then measured along the base line

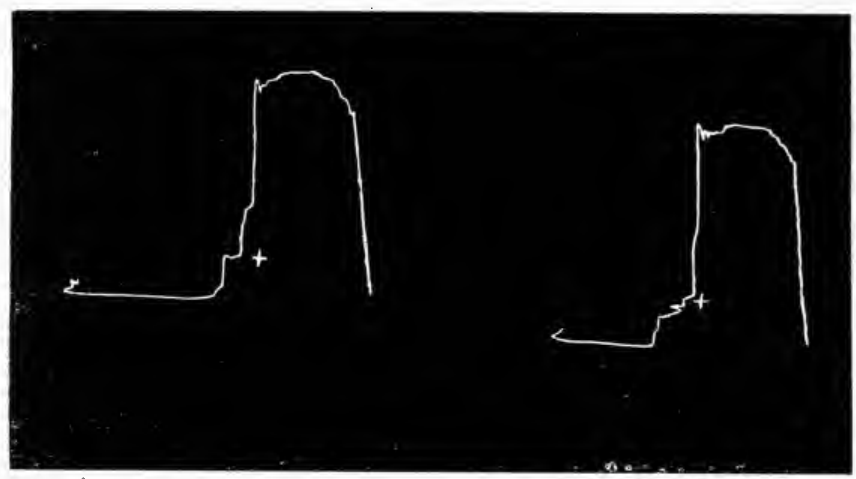

Fig. 32.-Odontograms of Deciduous Teeth.

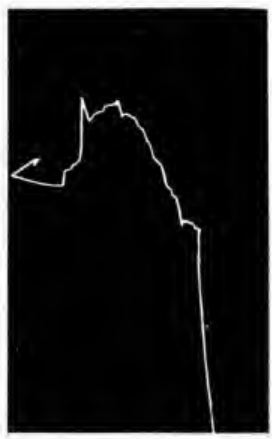

Fig. 33.-Odontogram of a Sclerotic Tooth.

from the point where the enamel begins to the vertical line, it may be said that the thickness of the enamel is inversely proportional to this measurement.

The odontogram of a sclerotic (European) tooth is shown in Fig. 33 It will be noticed that it is intermediate in type 
between the malacotic and "native" tooth; the rise is a little more abrupt than in the malacotic tooth, and the imbrication waves are a little less marked.

Fig. 34 represents the odontograms of a dog's teeth. An abrupt rise is noticed, but there is a complete absence of imbrication waves.

A considerable number of odontograms have been obtained in this manner, and they all conform, with minor exceptions, to the above types.

In using the odontograph, it is necessary, of course, for the

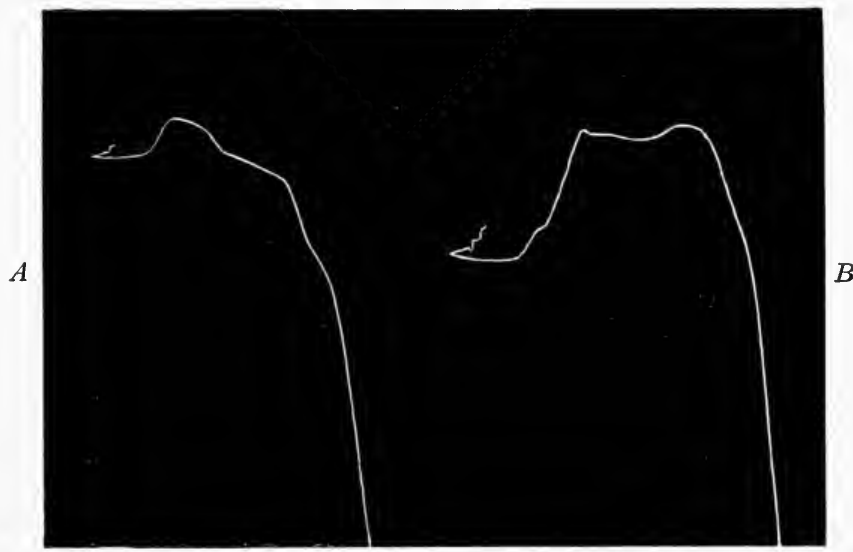

Fig. 34.-Odontograms of a Dog's Teeth.

$A$, Canine tooth; $B$, molar tooth.

sake of comparison, to know (I) the length of the lever, and (2) the ratio of the speed of the drum to that of the carriage, and only those odontograms obtained under similar conditions can be compared. For the odontograms here reproduced a lever of 2 feet in length was used, and the carriage was arranged to travel at exactly half the speed of the drum.

By means of this graphic method, therefore, are again confirmed the differences previously observed between the various classes of teeth. 


\section{THE ENAMEL SURFACE: ORIGIN AND SIGNIFICANCE OF THE IMBRICATION LINES}

THAт the imbrication lines are not artifact or acquired is proved by the facts (I) that they are best marked on unworn surfaces and on unerupted teeth, of which a considerable number, both "native" and European, have been examined; and (2) by their presence on those teeth, native and otherwise, which have a covering of salivary calculus.

(Fig. 35, a photomicrograph from the naturally polished surface of a canine tooth, shows " artifact" lines, which are obviously of a very different nature.)

It is inconceivable, from their practically universal presence, that they are due to any hypoplastic defect. They therefore must be regarded, at least in "native" teeth, as physiologically developed structures.

It is of importance in this connection to note the very close relationship between the imbrication lines and the "striæ of Retzius "; this is shown quite distinctly in Figs. 23 and 24.

Fig. 23 being photographed by reflected light, the striæ of Retzius appear light (but it will be noticed that Schreger's lines are dark).

In Figs. 24 and 37 they are of the usual dark colour.

It will be noticed that each ridge appears to be formed by the termination of one of the "strix of Retzius," and that each furrow or depression coincides with the termination of a light striation of enamel separating the dark striæ. Further- 
more, both the frequency and length of the surface ridges would seem to be directly dependent on the angle of incidence which the striæ make with the surface.

Thus in the cervical portion the angle of incidence is greater, and the surface ridges are both numerous and short; towards the occlusal surface the angle of incidence becomes progressively less, and the ridges longer and fewer, until the striæ

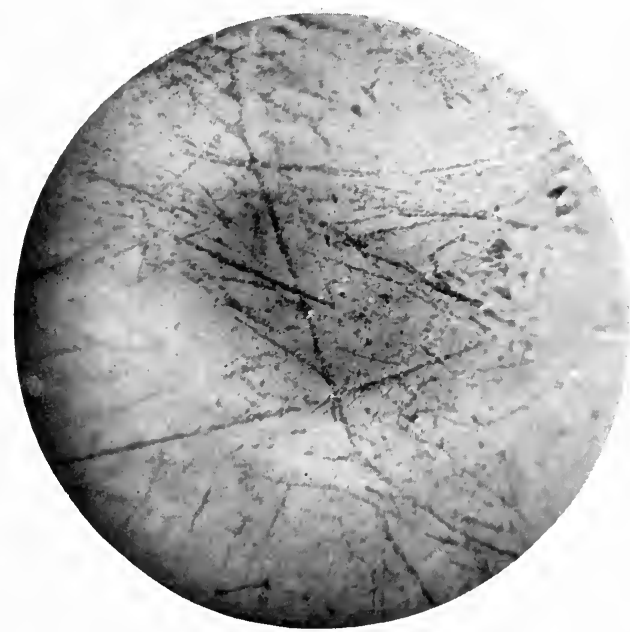

Fig. 35.-Artifact Lines on Canine Tooth, "stained" with GRAPHITE.

become parallel with the surface, and the ridge ceases to be distinguishable as such, but forms part of the general contour of the tooth (Fig. 36).

And, conversely, when the ridges cease to be apparent, the striæ of Retzius also cease to be so marked, although remaining distinctly visible. Again, in those teeth, animal and human, from which the striæ of Retzius are absent, the imbrication lines are also absent. The absolute interdependence of imbrications and stria upoin each other is thus apparent. 
I have therefore termed these lines on the enamel surface imbrications or imbrication lines, since the whole appearance is so suggestive of stratification and imbrication as to give reasonable ground for the belief that this is the method of the formation of ridges, furrows, and striæ. This is in accord with Kölliker's and Walkoff's belief that all striation in enamel

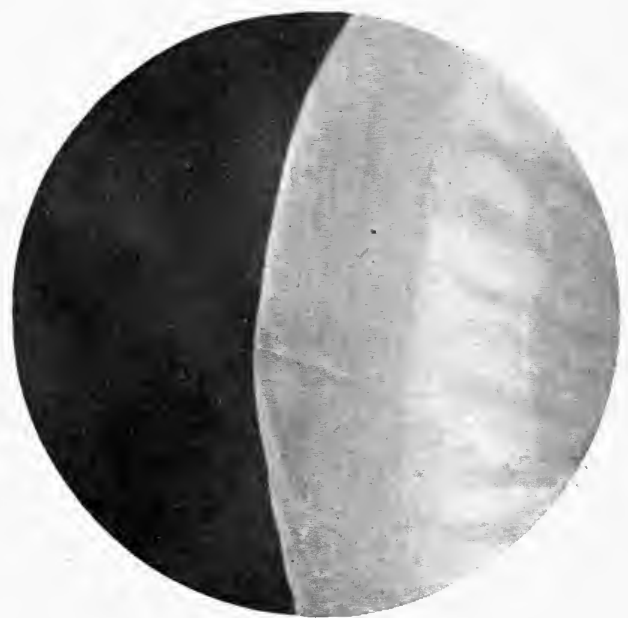

Fig. 36.-Cessation of Imbrication Lines when Striations become Parallel to the Surface: Coronal Region of Unworn Molar ТоОтн.

is due to the deposition of lime salts in strata. It is opposed to the theory that the striæ of Retzius are due to pigmentation only.

Leon Williams has proved that enamel rods pass through some varieties of striæ without break in continuity. This latter is undoubtedly true of some striæ (those in the occlusal part of the enamel especially, which do not make any outcrop on the surface), but does not affect the argument, and is only analogous to what is frequently observed in dentine and cement when increments are added. 
If the striæ of Retzius were due to pigmentation, they should appear the same both by reflected and transmitted light-i.e., dark; but, as has been pointed out, by reflected light they appear white, and the intermediate substance dark. This can only be accounted for by the striæ possessing a greater optical density.

Difference in physical density may be proved by examining suitable sections by polarized light. The following simple

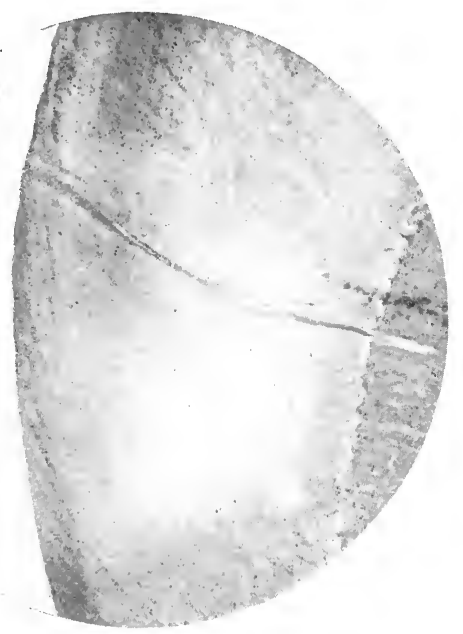

Fig. 37--Striak and Imbrications: " Point of Contact" Region

experiment gives an analogous probability that the "brown" lines are due to an increase in density.

A number of overlapping films of pure "Chinese white" (zinc oxide) and water were painted on a microscope-slide and examined under the microscope. The result is a brown striation, in appearance closely resembling that of the brown striæ of Retzius when viewed by transmitted light (Fig. 38); but by reflected light the brown striation becomes white, and the intervening film dark (Fig. 39). These two photo- 
micrographs were made without taking the slide from the stage.

We are therefore bound to conclude that such "striation" in enamel is due to physical differences in refraction, and not to pigmentation at all (except in pathological cases).

There appear to be two varieties of lines or stria-one which is caused by the granularity or increased optical density of

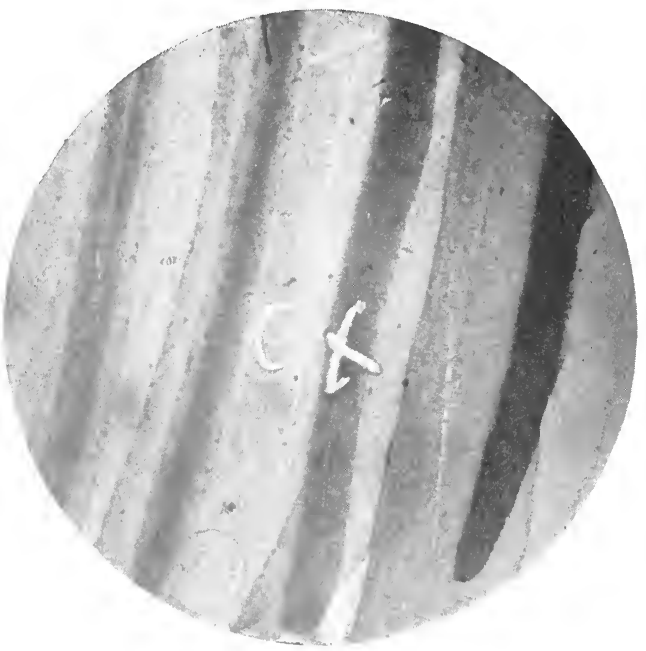

Fig. 38.- “Brown Striations" $(x)$ produced by Over. LAPping Films of Chinese White.

Viewed by transmitted light.

the prisms themselves, and a second which is caused by an increased amount or a differing disposition of the cement substance.

The former variety are found chiefly in the occlusal half of the enamel, and constitute those striæ which run more or less parallel to the surface of the enamel, and do not make any actual " outcrop" on the surface.

These strix are caused entirely by an increased optical 
density or granularity of the prisms themselves; they are of varying intensity, thickness, and frequency, and their margins are comparatively ill-defined.

The second variety of striæ are seen mostly in the cervical portion of the enamel, and constitute those striæ which make an outcrop on the surface. There may be merely an increased amount of cement substance on each side of the

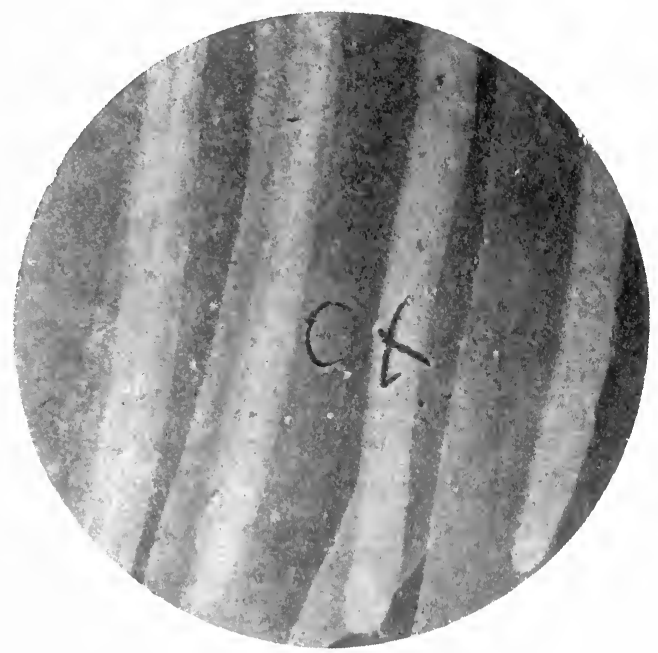

Fig. 39.-The Same as Fig. 38, But viewed by Direct Illumination. The "Brown Strie" have become White.

prisms, corresponding to a constriction of the latter, or the cement substance may pass right across the prism in a narrow band. This can only be observed by fine focussing in sections not too thin, and hence cannot be adequately represented in a photograph.

It is frequently stated that this is only apparent when the section is cut at a "certain angle"; this I have not found to be so. It would, I think, be truer to say that it only occurs in enamel from a "certain position "-i.e., the cervical half 
or one-third, and then only near the surface. Here, too, after this variety of striæ the enamel prisms frequently suffer deflection from the original course, and in human teeth at least this deflection is practically always towards the occlusal surface-i.e., in the direction in which there is most room in the crypt.

Horizontal sections do not show any lateral deviation of the prisms; neither on fine focussing through thicker sections are the prisms seen to deviate in more than one direction.

\section{Hypothesis.}

The only rational solution of such appearances is that imbrications and striations of all varieties are to be regarded as evidence of checks in the secretive function of the ameloblasts.

As regards the nature of the check to the ameloblasts above referred to, the writer believes it to be due to the pressure of the bony walls of the crypt in which the tooth is developingi.e., the resultant of two forces of evolution:

I. The hereditary tendency of the ameloblasts to continue functioning to a certain definite extent.

2. The limiting force of environment, expressed as a diminution in the size of the jaws-i.e., the result of altered habits of diet, and also to some extent of natural selection.

The cap of enamel commences to be formed over the " cusp " of the dentine, and gradually extends down to the cervix, but the full complement of enaniel is laid down at the occlusal surface long before it is complete in the cervical region; hence it is obvious that atrophy of the ameloblasts in the occlusal region must occur, whilst those in the cervical region continue their function.

This process of atrophy of an increasing number of " occlusal " ameloblasts and active secretion by a decreasing number 
of cervical cells must continue until development is complete. Reference to Fig. 40 explains this diagrammatically.

Enamel formation commences at $A$, and continues concentrically until $B-C$ is reached; the cusp of the occlusal surface is then fully formed, and the ameloblasts atrophy in that region-i.e., from $B$ to $D$-but continue to function from $C$ to $D$.

The fact of a number of cells of the enamel organ thus atrophying will have an effect on the remainder. If atrophy of a portion of an organ be gradual, the effect is probably to increase the functional power and secretion of the other cells; if more sudden, as it would be by the ameloblasts being driven against the unyielding walls of the crypt, the check would be communicated to the rest of the organ-i.e., the remaining ameloblasts. The result would undoubtedly be the formation of enamel of an altered density, or a sudden diminution in the size or a cessation of the prisms-both of which would give a well-marked line or striation.

The granularity or increased optical density of the prisms is probably an expression of a minor check to development,

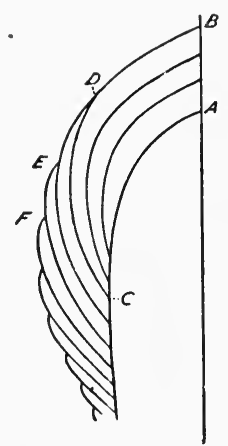

Fig. 40,-DIAGRAM TO EXPLAIN HYPOTHESIS AS TO ORIGIN OF STRIE AND IMBRICATION LINES. and, as we have seen, this variety of striæ is only seen in that part of the enamel which is formed before the occlusal portion has reached its maximum development-i.e., when the disproportion between the requirements of the developing tooth and the available space has not become acute.

The other variety of striæ occurring after the latter event is caused by a more sudden and acute atrophy, and hence even those ameloblasts which do not atrophy temporarily cease to function to their full and normal_extent, the result being a 
marked constriction in the prisms, or a line of cement substance passing obliquely across the prism.

Let us now refer once more to Fig. 40. The process of atrophy occurs again at $E$ and $F$; i.e., the cells between these two points atrophy, and so give rise to a well-marked ridge of enamel on the surface where calcification is continued, and a striation passing inwards as far as the dentine.

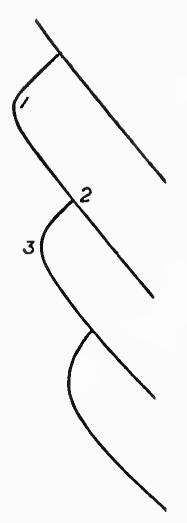

FIG. 4I.-DIAGRAM

TO ILLUSTRATE

THE ORIGIN OF

I M B RI C ATIO N LINES.

$\mathrm{I}-2=$ number of cells

atrophied ; $2-3=$ time between each " atrophy." That is to say, according to this hypothesis the distance between "ridge" and "furrow" (I to 2 in Fig. 4I) is a measure of the number of cells which atrophied; and the depth of each "ridge" ( 2 to 3 in Fig. 4I) is a measure of the time between each "atrophy." Both of these diminish towards the cervix, where either less enamel is "required " or there is less room for its development.

It would thus appear that an atrophy of ameloblasts occurred fairly regularly. There may be, too, some significance in the fact that the depth of each imbrication or ridge ( 2 to 3 in Fig. 4I) approximates very nearly to the length of the ameloblasts in that region; for instance, the former measure 0.024 millimetre, and the latter 0.025 millimetre, in the region half-way between the cervix and the occlusal surface.

The above hypothesis receives support from a microscopical examination of some forms of hypoplastic enamel, especially that form in which the lesion occurs after the completion of the enamel tip. This condition is admittedly caused by a gross arrest of the function of the enamel organ, usually through some systemic disease. If now the imbrications and striations are normal expressions of minute arrests, in gross 
arrests we should expect to see gross imbrications and striations, which is exactly the case (see Fig. 42).

Mention has been made of the presence of imbrication lines on the teeth of some of the lower animals. The factor which seems to determine their presence or absence is the relative length of the circumference of the cervix to that of the occlusal surface. If the former exceeds the latter, then the imbrication lines are absent; but, in proportion as the occlusal surface

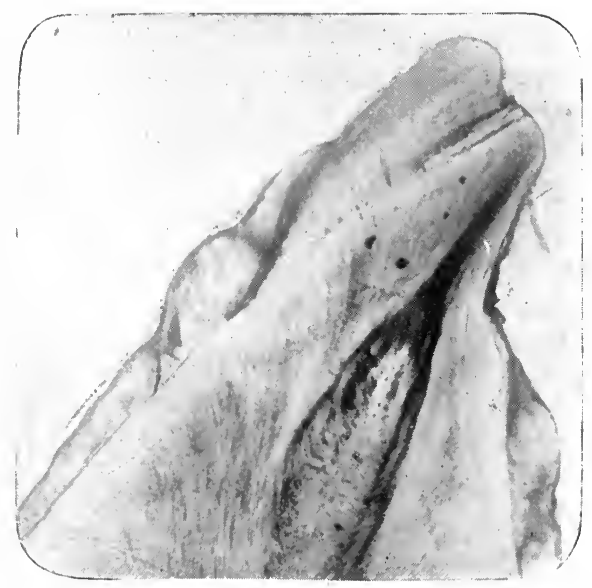

Fig. 42.-Vertical Section of a Hypoplastic Tooth, showing Exaggerated Imbrications obviously due to Gross Arrests of Development. (Dental Cosmos, October, r9i3.)

becomes more flattened and of greater circumference, so the imbrication lines appear, at first only in the cervical onethird, but extending upwards towards the occlusal surface until they reach their maximum development in Hylobates and civilized man.

It would appear in cases where imbrication lines are absent that the enamel is laid down in the manner indicated in Fig. 43, and that no atrophy of occlusal cells takes place on account of the relative size and shape of the dentine papilla and the 
dental crypt; i.e., there is no diminution in the size of the jaws, and therefore no check to development. In support of this is the fact that in those teeth in which the imbrication lines are absent the striæ or indications of atrophy of the ameloblasts are also absent. This would account for the absence of imbrication lines on the deciduous teeth, and also for their less marked development on "native" teeth ( $c f$. Figs. 2I, 3I, and 32).

In effect, if this hypothesis be true the facts may be stated

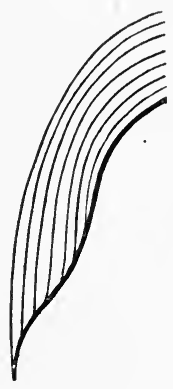

Fig. 43.-Diagram ILLUSTRATING THE METHOD OF ENAMEL FORMATION IN TEeth WHOSE C ERV I C A L CIRCUMFERENCE IS GREATER THAN THEIR OCCLUSAL. thus: The dimensions of the approximal alveolar portions of the jaws before and while the deciduous teeth are in position are governed by hereditary forces, but the dimensions of the body of the jaws are governed also by the forces of environment. If the latter oppose the former, then the developing permanent teeth in their crypts will be crowded (see Figs. 44, 45 , and 46); this leads to such modifications in their structure as must considerably lower their resistance to the attacks of caries.

The remedy would appear to be perfectly obvious:-To increase the beneficial environmental forces by providing for the development of those muscles of mastication which tend to expand the jaws; to guard against the unnecessary use of those muscles of trituration and deglutition which contract the jaws; and to encourage by every means possible the growth and strength of the muscles of articulation, speech, expression, and emotion, which, as we have seen, tend to produce the characteristic rounded moulding of the normal human jaws and dental arches; lastly; and by no means least, to properly treat all cavities of the deciduous teeth, the pres- 


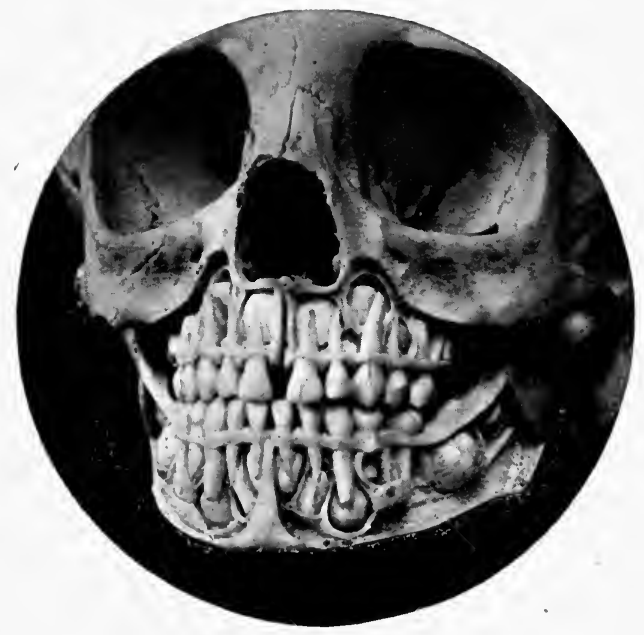

Fig. 44.-Dissected Jaws of a Child, illustrating the Amount of Crowding of the Developing TeEth Which May EVEN OCCUR WHEN THERE IS NO CARIES OF THE DECIDUOUS TEETH

The permanent upper lateral incisors_are nearly crowded out of existence.

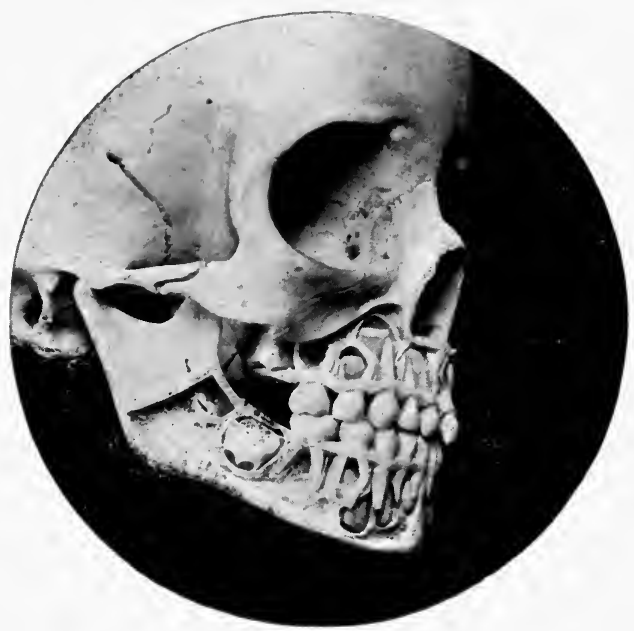

Fig. 45.-Similar to the Above. 
I 00

ence of which is such a frequent cause of non-expansion of the jaws and of crowding of the developing permanent teeth in their crypts.

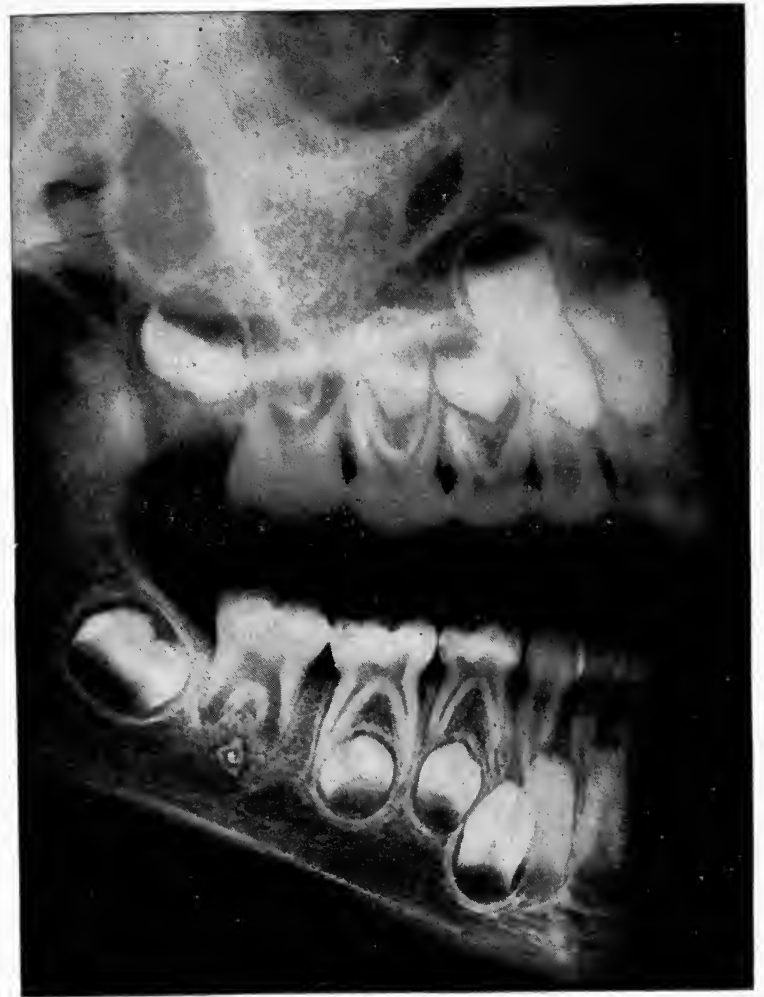

Fig. 46.-Skiagram of Jaws of a Boy Aged Six.

Note how small is the amount of room available for the development of the permanent premolars.

(From Symington and Rankin's "Atlas of Skiagrams," with permission of Messrs. Longmans, Green and Co.) 
CHAPTER IX

THE ENAMEL SURFACE: DEPARTURES FROM THE "NORMAL" STRUCTURE, AND THEIR PATHO. LOGICAL SIGNIFICANCE

IF the enamel surface of "native" teeth be regarded as " normal," then that of civilized and recent teeth must as a general rule be regarded as abnormal, for in the vast majority of cases the two are materially different.

The collection of skulls previously mentioned as being examined was in a disarranged state, and thus one had no direct reason to know to what race a skull belonged until reference was made to the catalogue afterwards; and it was found that one was soon able to distinguish native from European skulls merely by the appearance of the enamel under the microscope.

In the teeth of uncivilized races the surface of the enamel is lustrous-i.e., reflects much unbroken light from its surface; the imbrication lines are often difficult to make out, on account of their being comparatively feebly formed, and also on account of the fact that the graphite does not adhere to the enamel as it does in malacotic teeth, and thus the surface is difficult to "stain."

In a certain number of cases, when the light was reflected at the right angle, the outlines of the prisms were readily discernible. They were not stained, since they are more readily seen without the graphite than with it. The "cement substance " merely appears a little darker-i.e., does not reflect so much, or absorbs more light—-than the enamel prisms, just as it does in sections of enamel viewed by reflected light. 
In no case should the centre of the prisms be differentiated. The points that strike one in the examination of the enamel surface of the teeth of civilized races are the comparative lack of lustre, the ease with which the surface ridges can be made out with the naked eye, the facility with which they stain by the graphite method, the evenness of illumination when examined microscopically by reflected light (instead of a brilliant pencil of light from one point); and microscopically the "ridges" are seen to be both wider and deeper when

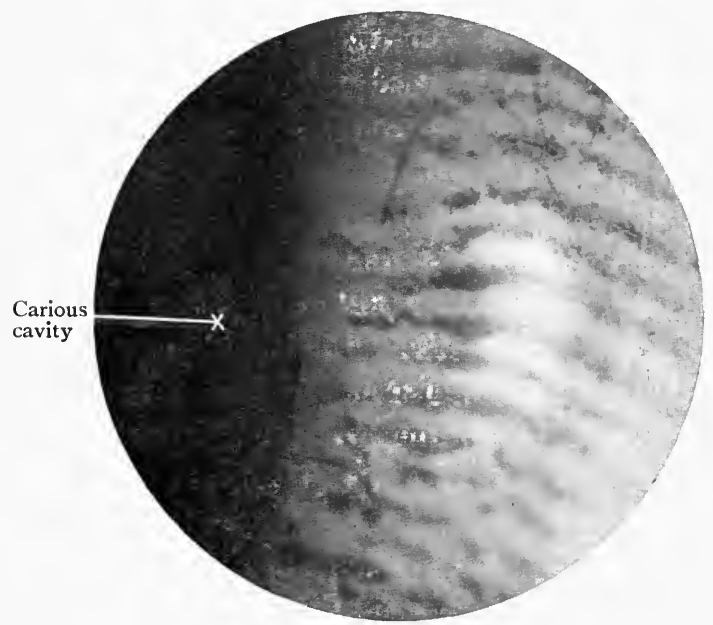

Fig. 47.-Imbrication Lines on Lower Incisor of Malacotic TYPE.

examined by reflected light, and also in the ordinary way by sections. And it is a most significant fact that the more " susceptible" the mouth from which the teeth are taken, and the more malacotic in type the tooth is, the more marked and wider and deeper are the imbrication lines. This is well shown in Figs. 47 and 48. Both photomicrographs are from the mesial, and therefore unworn, surfaces of lower incisor teeth. Fig. 47 shows the imbrication lines on a tooth removed 
from a young girl of eighteen with excessive dental caries. Fig. 48 shows the imbrication lines on a similar tooth from a woman of sixty, whose remaining teeth were quite sound, but were extracted for looseness. The tooth in Fig. 47 had succumbed to caries in ten years; the other (Fig. 48) had resisted for over fifty years. I do not state these facts as being absolutely cause and effect, but that they are related to a considerable extent seems indubitable.

Only very occasionally do the outlines of the prisms show

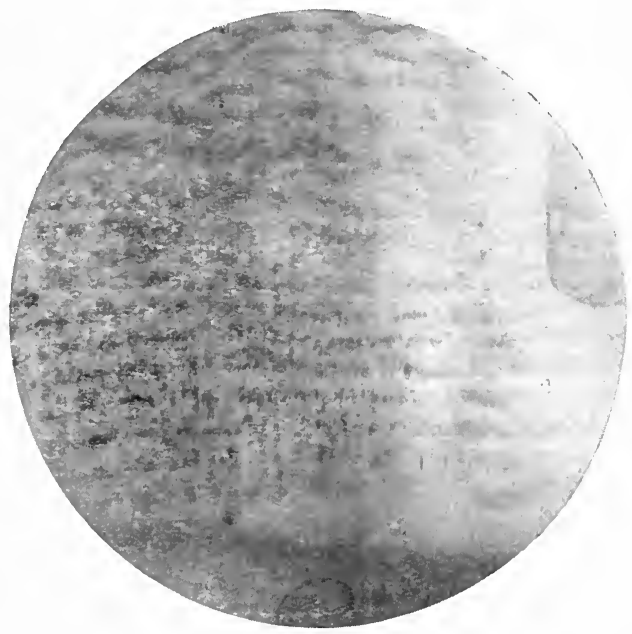

Fig 48.-Imbrication Lines on Lower Incisor of Sclerotic TYPE.

dark in unstained malacotic teeth. It is much more usual for the centre of the prisms to stain dark by the graphite method, as seen in Figs. 49 and 50. This means, of course, that the end of each prism is depressed, and in the hollow so formed the graphite has lodged.

In some cases, by throwing a beam of light along the surface of the enamel, the centres of the prisms appear dark in a light 
hexagonal ring even when no graphite is used. This seems to show that the dark areas are depressions unilluminated.

The average diameter of the prism ends stained with graphite is 0.00375 millimetre, and the average diameter of enamel prisms in this situation is 0.0057 millimetre (measured in sections by transmitted light). This should leave 0.002 millimetre as the thickness of the "unstained" rings, which is

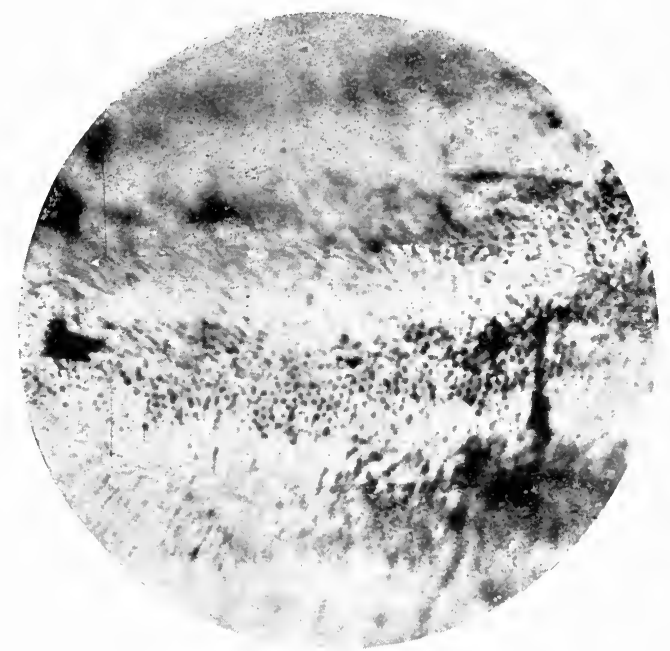

Fig. 49.-Imbrication Lines and Depressed Prism Ends stained with Graphite.

exactly what they do average. These unstained margins of 0.002 millimetre are too wide to be composed of cement substance only, and probably comprise the outer part of the enamel prism as well. Thus it would seem that in such cases the ameloblasts have ceased functioning before the prisms were quite complete, and have left the centres-perhaps Tomes's processes-uncalcified. I have found such appearances only in malacotic teeth. 


\section{Sclerotic Teeth.}

So far chiefly "native" and "malacotic" teeth have been contrasted. Sclerotic teeth of civilized races may be said, as far as histological appearances go, to fall between these two classes, but much nearer the "native" in type than the "malacotic." Much more light is reflected from the surface of a sclerotic than from a malacotic tooth, but not so much as

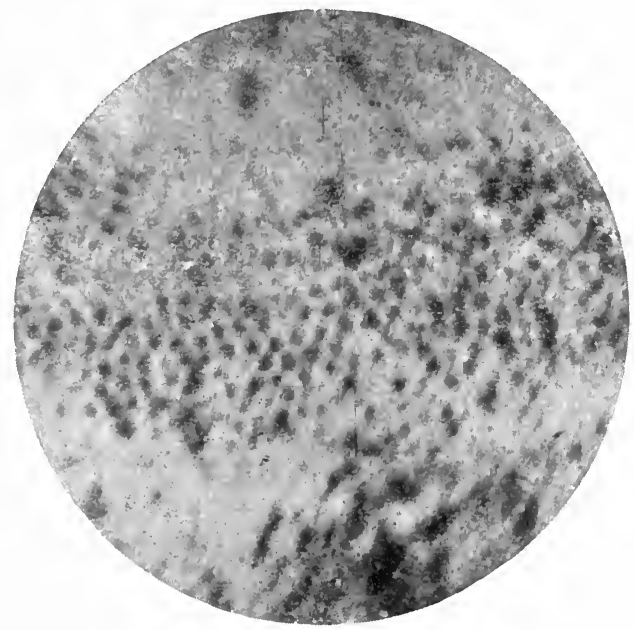

Fig. 50.-The Same as Fig. 49, But More Highly Magnified.

from a native tooth. The imbrication lines vary in their distinctness in different teeth of this class, but they are rarely so well marked as in the "malacotic" type. I have not observed any depression of prism ends in any sclerotic tooth.

\section{Pathological Fissures of the Enamel Surface.}

In addition to the increased development of the imbrication lines in malacotic teeth, there are three classes of fissures met with, and which I have classified as-(I) Calcarine, (2) Sinuous, (3) Deep. 
Those of the first kind apparently originate at the summit of the ridges, are broad, short, triangular in outline, and very numerous, and may be designated as "calcarine" fissures. They are most marked on the mesial and distal surfaces, and on those points where the ridges are most marked--i.e., at a point one-half of the distance between the cervix and the occlusal surface. Fig. $5 \mathrm{I}$ is an example of this class of fissure, stained, of course, by the graphite method.

Fissures of the second class are not so numerous nor so fre-

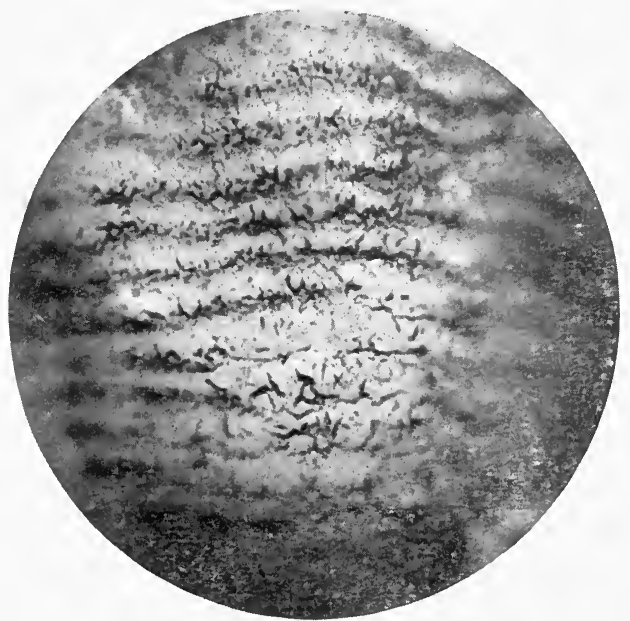

Fig. 51.- "Calcarine" Fissures on the Surface of a Malacotic Molar.

quently met with, are longer and thinner, and bear no relationship in origin to the ridges, many of which they cross. Fig. 52 illustrates these fissures, which might be termed "sinuous," on account of their thin wavy outlines.

Both of these classes of fissures are best demonstrated by very light staining with graphite; they are invisible without staining, and heavy staining obscures them. I have not seen them in other than malacotic teeth. 
Those of the third class, classified above as " deep," run usually in the direction of the long axis of the tooth, and vary considerably in length. They penetrate deeply through the enamel, and occasionally even into the substance of the dentine. Fig. 53 is an example of this kind of fissure stained with silver nitrate. That these latter fissures are not artifact is proved by the manner of preparation. The teeth were fixed in formalin immediately after extraction, washed and trans-

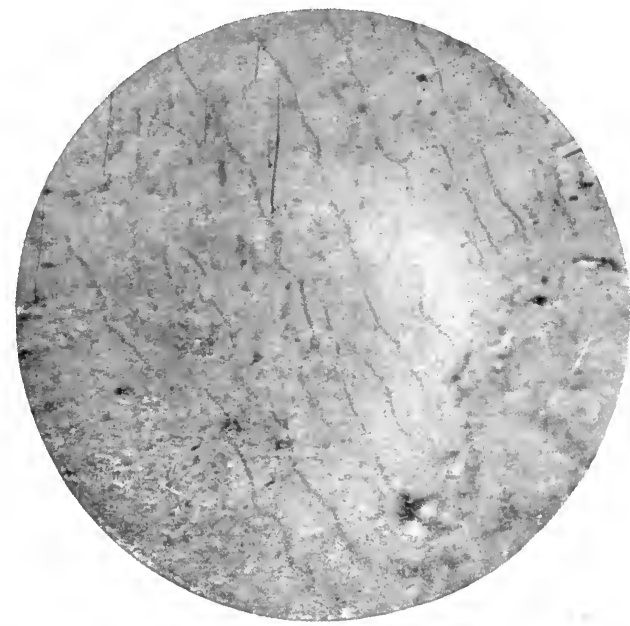

Fig. 52.- Sinuous Fissures from a Lower Incisor.

ferred without drying to a Io per cent. solution of silver nitrate for twelve hours, washed again in running water for one hour, placed in the sunlight for one day, and then ground down slowly and examined frequently, so that the fissures could be traced from the surface inwards. Fig. 53 (an upper premolar) has been prepared in this manner.

This class of fissure is, of course, frequently seen in ordinary ground sections of enamel, but there is always the uncertainty as to their having developed during the proc ess of grinding. 
As regards their origin, undoubtedly many are caused by traumatism, or possibly thermal changes; but that some are developmental cannot be doubted, and may be due to the persistence of some portion of the enamel organ.

Figs. 54 and 55 show such a cleft or fissure, stained with silver nitrate before cutting, and taken from the distal surface of a molar tooth.

The enamel prisms on either side of the cleft are quite

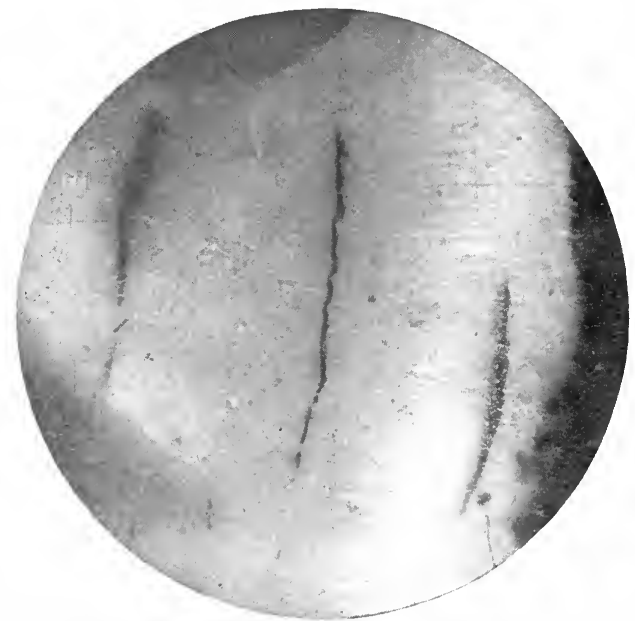

Fig. 53.-“Deep" Fissures in an Upper Premolar.

granular and indefinite in outline, and towards the surface both they and the stratification striæ show distinctly a certain amount of retroversion--as though there had been something retarding the outward movement of the enamel organ at this point.

\section{Opaque Spots.}

But little information has been gained from the examination by reflected light of "opaque spots" caused by initial caries when these have reached the dull stage, because of the 


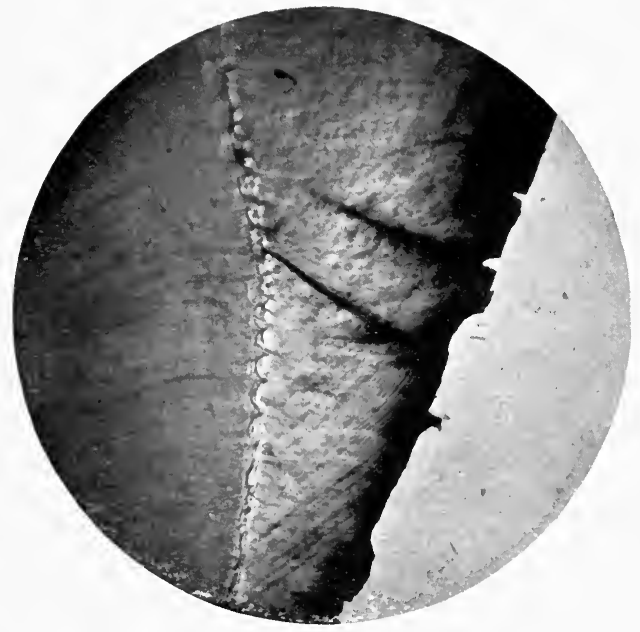

Fig. 54.-Developmental Fissure in the Enamel near the Cervix of a Malacotic Molar (Low Power).

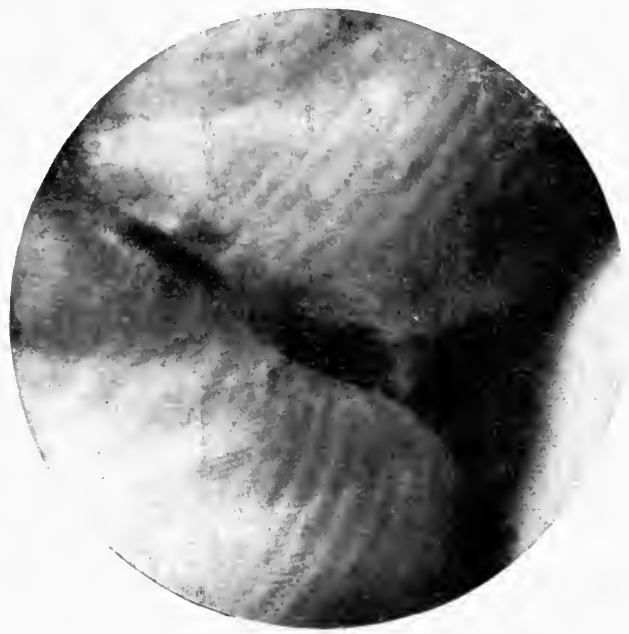

Fig. 55.-The Same Section as Fig. 54, More Highly Magnified. 
technical difficulties of staining and lighting. Such areas do not appear to possess any definite structure, but are composed entirely of highly refracting globules when unstained, and they take the graphite stain so deeply as to obscure all structure. When, however, calcarine fissures are present, caries can be seen attacking these in advance of the surrounding tissues.

\section{Conclusions.}

That these departures from the normal structure are potent factors in lessening the resistance of the teeth to caries cannot be doubted. They must favour considerably the adhesion of foodstuff and micro-organisms; they expose a larger and more vulnerable surface to the action of the acid produced; they negative both mechanical and physiological prophylactic measures; and the fissures which have been described, since they allow of the penetration of silver nitrate and graphite, must permit the penetration of lactic acid, if not of carbohydrates. In fact, ceteris paribus, it may be quite logically inferred that the amount of graphite which an enamel surface retains is a measure of the amount of foodstuff it would tend to retain, and a demonstration of the situations of its retention. Added to which is the demonstrable fact that teeth so affected are not infrequently likewise the subject of caries.

On the other hand, it may be thought, there is the common clinical experience as to hypoplastic teeth being by no means always subject to caries. Such teeth (non-carious) examined by the method I have indicated usually show the enamel surface to be free from any minute defects and to have a very fair lustre; even the surface ridges are frequently absent from the hypoplastic areas, because the striæ of Retzius, or " incremental lines," run parallel to the surface, thus proving that the check to development occurred when the cap of enamel was only partly formed, and that normal enamel has been laid down afterwards, but that the rate of deposition at that par- 
ticular spot has not increased sufficiently to restore the contour.* This is also borne out by the examination of sections of such teeth by transmitted light. The inference from this, of course, still further strengthens the relationship between minute structural defects and caries. The defects which are gross and obvious to the naked eye are not those which, as a rule, lessen the resistance of a surface to caries.

* Such defects may be termed " healed hypoplasia." 
PHYSICAL PROPERTIES OF THE ENAMEL SURFACE

\section{Permeability of the Enamel Surface.}

IT is generally supposed that the surface of enamel is normally impermeable to fluids and stains; in fact, Gabell and Austin* state that " it is quite impossible to stain enamel." This, however, cannot be accepted as an absolute fact. No doubt it is extremely difficult to stain healthy enamel with the ordinary stains; but a ro per cent. solution of silver nitrate will be found to stain the surface of all enamel, and, what is of more interest and importance, it stains to a varying degree in different teeth; and not only does the intensity of the stain vary on the surface of the enamel, but it penetrates to a varying depth in different teeth. This having been ascertained whilst carrying out other experiments, the subject was investigated in the following manner :

Perfectly sound teeth were placed in a ro per cent. solution of silver nitrate for twenty-four hours, and then exposed to bright sunlight for a day. They were then washed in salt water, and sections cut in the usual way. The depth of penetration of the silver nitrate was measured by means of stage and eyepiece micrometers. Measurements were taken inwards from the enamel surface at intervals of I millimetre round the periphery of the enamel. The sum of these measurements divided by the number of them gave the average for each tooth. In this manner it was found that the average

* "Materia Medica for Dentists," p. 207. 
penetration for malacotic teeth was 0.27 millimetre (or 16.3 micrometer divisions), and for sclerotic teeth 0.13 millimetre (or $7 \cdot 8$ divisions) - that is to say, the stain penetrated twice as far on the average in the malacotic as in the sclerotic teeth.

The penetration is more regular in the latter variety, the maximum measurement being 0.25 millimetre. In malacotic teeth the stain tends to penetrate very deeply in places, whilst maintaining a general average penetration greater than that of sclerotic teeth. The average maximum penetration in such teeth was 0.83 millimetre, or 50 divisions. These maxima penetrations are usually associated with some fissuring of the enamel.

Maori teeth treated in a similar way invariably showed a less dense superficial staining, and the penetration was in some cases scarcely observable, the average penetration being only 0.07 millimetre, or 4.5 divisions.

In all varieties, wherever the enamel surface has been damaged by abrasion, fissures, or fracture, the stain penetrates more deeply than normally. Figs. 56 and 57 illustrate the penetration of silver nitrate in malacotic and sclerotic teeth respectively.

The degree of permeability of the enamel surface also bears an inverse ratio to the length of time a tooth has been erupted. This is illustrated by the following cases:

I. Three teeth-an unerupted lower third molar, an erupted upper third molar, and a lower premolar-all perfectly sound and from the same patient (aged twenty-two), were examined by this method, with the following result:

Tetth.

Unerupted third molar

Erupted third molar years' use)

Premolar (half-sclerotic type, eleven years' use)
Amount of Penetration.

$0.83 \mathrm{~mm}$. (or 50 divisions).

$0 \cdot 42$, (or 25 ,, ).

O.I8 , (O1 I.I ,, ). 


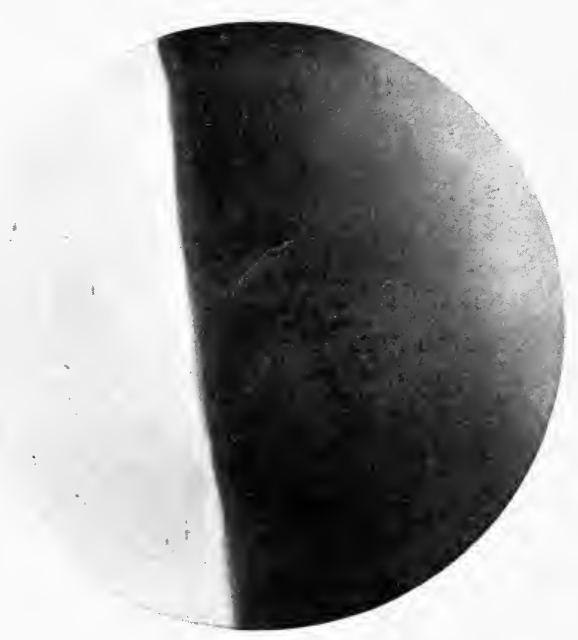

Fig. 56.- Photomicrograph Showing Amount of Penetration of Silver Nitrate in Malacotic Enamel. (2 ObJ., No. 2 EyePIECE )

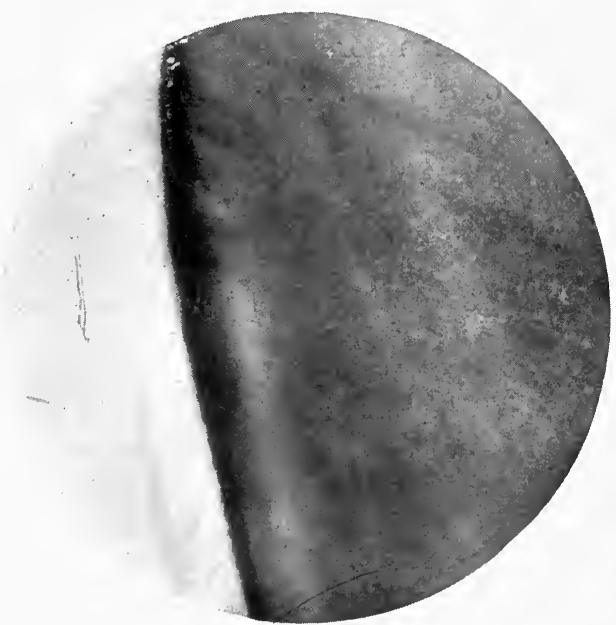

Fig. 57 - Photomicrograph showing Amount of Penetration of Silver Nitrate in a Maori Tooth. (2 ObJ., No. 2 Eyepiece.) 
2. The following teeth were taken from different individuals :

Teeth.

Unerupted third molar

Erupted third molar

Molar from young adult

Molar from old person
Amount of Penetration.

.. $\quad$. $0.50 \mathrm{~mm}$. (30 divisions).

.. .. $0.30 \quad, \quad(20 \quad, \quad)$.

.. . 0.25 ", (I5 ,

. . . 0.16 ,, (10

The density of the stain in the last tooth was very much less than that in the third molars.

3. The following teeth were taken from the skull of a Maori child aged about sixteen years :

\section{Teeth.}

Unerupted third molar (incomplete)

Erupting third molar (complete) ..

Second permanent molar (thre years' use)

First permanent molar (nine years' use) 0.08 , ( 5 ,").

A point of importance to be noticed in the above groups is the comparative equality of penetration in the unerupted molars of both Maori and European. This would seem to point to the fact that the cause of the subsequent difference in the permeability of the various enamels is not entirely a developmental one, but rather is a property acquired largely after eruption.

Comparing the second permanent molar of the Maori with the premolar of the first group, it is seen that in two years the Maori tooth has become as impermeable as the European tooth has in eleven years. Such inferences do not, I am aware, agree with the accepted tenet that enamel once formed cannot possibly undergo any physiological alteration; but it is difficult to see what other logical conclusion can be drawn from the facts, especially when these are supported by others, as will subsequently be shown.

The stain penetrates at first along the interprismatic substance, and then seems to spread laterally into the prisms. 
Fig. 58 is a photomicrograph of a transverse section of superficial enamel treated by the above method. It is seen that the interprismatic substance is stained darkly, but the prisms are not. In Fig. 59 the stain is seen clearly running along between the prisms, without much invasion of the latter.

\section{Nature of the "Staining."}

It is perfectly obvious that not all that is stained by the nitrate of silver is organic matter, since the prisms themselves

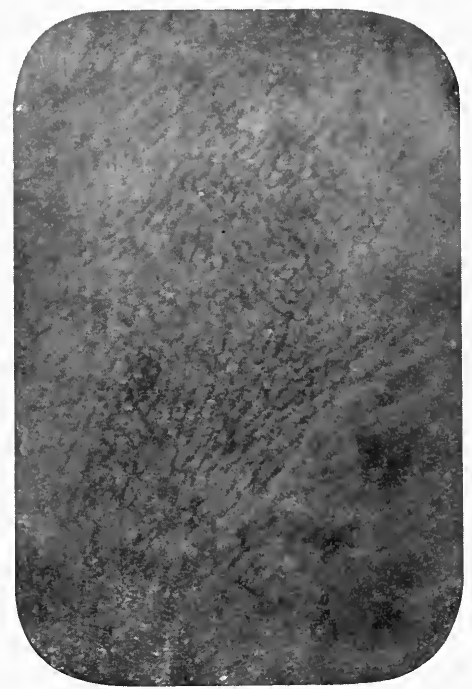

Fig. 58.-Transverse Section of "Surface" Enamel, showing the Interprismatic Substance stained, but not the Prisms THEMSELVES.

are stained; and I am not prepared to prove at present that any of the stained material is organic matter, though I think it is extremely likely that this will be found to be so, and for the following reasons:

r. It is well known that silver nitrate is a selective stain for the organic intercellular substance in other tissues. 
2. Hoppe-Seyler's analyses show a larger proportion of organic material in "infantile" enamel.

3. The enamel of a malacotic tooth was heated in an electric furnace, so that the organic matter (if any) would be destroyed. It was then treated by the silver nitrate method, and the degree of penetration of the stain found to be very much lessened, being only 5 divisions, or 0.08 millimetre.

On the other hand, the staining may be a kind of adsorp-

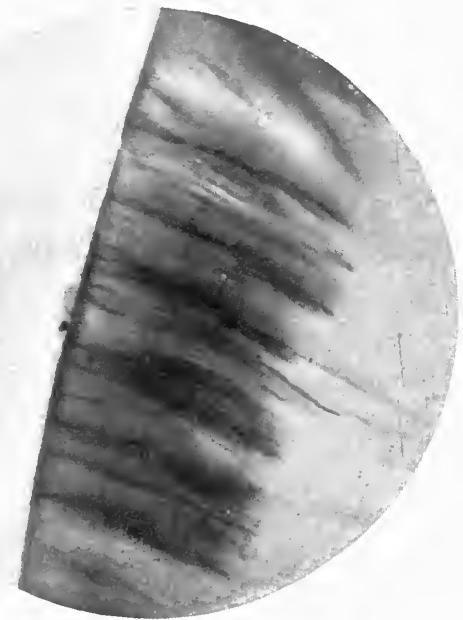

Fig. 59.- -Vertical Section of Enamel, Showing the Silver Nitrate penetrating inWards between the Prisms.

tion, ${ }^{*}$ and the silver precipitated in microscopic interstices. This, however, for the present does not very much affect the argument. The fact remains that a fluid carrying a solid in solution can pass into the outer layers of enamel, and that the solid is there fixed either by chemical combination or by

* The property of adsorption may be ascribed to some affinity between the molecules of the imbibing substance and of that imbibed. The retention of a gas or colouring matter by charcoal, of water by the opal, or pepsin by fibrin, are examples of such action. 
precipitation. The internal enamel surface at the amelodentinal junction does not seem to be permeable, as is shown by the following experiment:

The root of a malacotic tooth was sawn through, and the pulp cavity well opened. The tooth was then sealed in a small cup of wax, so that the crown projected into the cup and the root was below. The cup was then floated on silver nitrate solution in the dark. After exposure to the light, the enamel appeared a dark blue, but on cutting a section it was found that the stain had penetrated deeply up to the amelo-dentinal junction, but had nowhere crossed it. This was repeated with various modifications, but always with the same result. From this two inferences may be drawn:

I. As was to be expected, no means of communication exist between the enamel and dentine whereby fluid containing salts in solution or any other protective substance might pass from the latter to the former.

2. That that portion of enamel which is formed first is not permeable, butthat portion which is last formed is permeable.

Recently, Dr. Theo. von Beust* has attempted to show that a communication does occur between the dentine and the enamel. He used alcoholic carbol-fuchsin by capillary attraction, and states that the enamel in the inner portions was stained, more especially along certain " fissures," which correspond, I think, to my " interprismatic spaces." $\uparrow$ I am unable to confirm Dr. von Beust's findings. Certainly in teeth treated in such a manner the enamel is stained very occasionally, but it is comparatively rarely, and very erratically, that it occurs. In my specimens the stain seems a weak diffusion or absorption throughout a small area of enamel (which might be less highly calcified than the remainder). The majority of my specimens show the dentine deeply stained right up to the enamel, but the latter perfectly white, and not stained in the least degree. But $I$ think it by no means follows that, because a highly volatile and deeply penetrating stain like alcoholic carbol-fuchsin may pass occasionally across the amelo-dentinal junction, serum or lymph would do the same. Figs. 6o and 6r are interesting in this respect, in that they show how deeply silver nitrate

* Dental Cosmos, June, rgr2.

$\dagger$ See Dental Cosmos, 19r3, p. 985. 


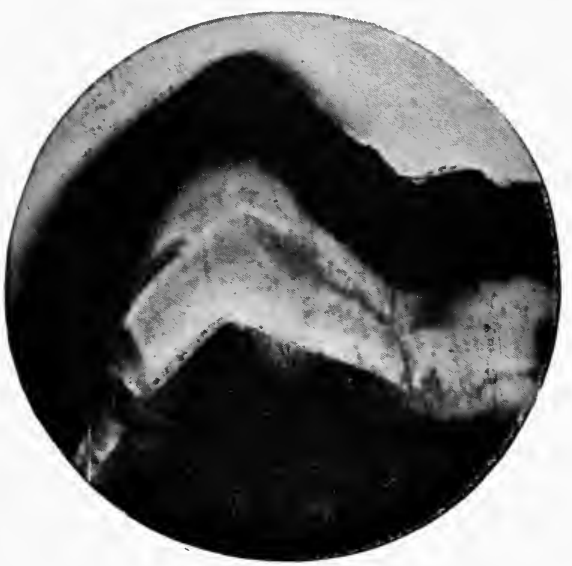

Fig. 6o.-Section of a MIALAcotic Tooth PREviously IMMERSED in Silver Nitrate Solution.

The stain has penetrated the enamel to a considerable depth from the surface, but no stain has passed from the dentine (itself deeply stained) to the enamel. (Dental Cosmos, October, r9r3.)

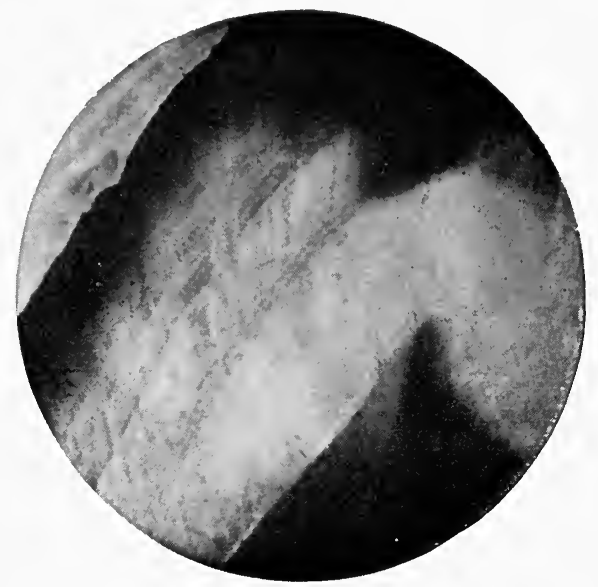

Fig. 6i.-A Similar Section to that shown in Fig. 60.

Enamel spindles are present, but these do not appear to have absorbed any stain. (Dental Cosmos, October, 19rz.) 
has penetrated malacotic teeth from the outer surface of the enamel, but that nowhere has the stain coming up the dentinal tubules crossed into the enamel. These are from sections which have been exposed to the light for many months, so that it cannot be said that the darkening of a small quantity of stain has not yet occurred.

\section{The Density of Enamel.}

The word "density" is used here purposely, not in a common scientific sense, as being identical with specific gravity, but rather in a literal sense of "compactness." It has already been shown that some enamel (more especially malacotic) is permeable to fluid to a certain degree, and that other enamel (sclerotic) is very much less permeable. Now, in order to obtain a correct idea of the comparative " density" of two bodies which vary in permeability, it is obvious that some other method than weighing them in water must be adopted. The ordinary specific-gravity method would, of course, give the correct specific gravity in each case, and it might be identical in each, but would not record the comparative " porosity"--compactness, or density with which the component parts were packed. For instance, spongy iron and ordinary iron have the same specific gravity when weighed in water, because water enters the porosities, and thus the specific gravity of the individual particles of iron is alone recorded; but this is no gauge of what may be termed the "mass density" of the spongy iron.

Precisely the same thing applies to enamel. For instance, suppose an individual and impermeable prism be separated out, and the specific gravity estimated. This would be precisely the same as that of the whole enamel were the prisms so tightly packed together that no fluid could enter. And suppose in the next case that the prisms were loosely packed; the fluid entering around them would again give the same specific gravity, but the mass density in the latter case would obviously be very much less than in the second instance. 
But suppose a fluid to be used that was incapable of penetrating between the prisms; then a true record of the mass density would be obtained.

The only fluid which can be relied upon for this purpose is mercury; and since enamel floats in this fluid, it became necessary to calculate the density by means of estimating the weight of the volume of mercury displaced from a pycnometer by pieces of enamel of known weight. The density is then obtained according to the following equations:

$$
\mathrm{D}=\frac{\mathrm{A} \times \mathrm{r} 3 \cdot 6}{\mathrm{~B}-(\mathrm{C}-\mathrm{A})} \text { and } \mathrm{B}-(\mathrm{C}-\mathrm{A})=\mathrm{E} ;
$$

Where $\mathrm{A}=$ weight of enamel in air.

$B=$ weight of bottle full of mercury.

$\mathrm{C}=$ weight of bottle full of mercury and enamel.

$\mathrm{E}=$ mercury expressed from pycnometer after inserting enamel.

(1 $3 \cdot 6=$ specific gravity of mercury.)

Enamel was obtained in the following manner: Sound teeth were placed in an electric furnace with a pyrometer attached, and heated to $300^{\circ} \mathrm{C}$. for three minutes. On pressure being then applied to the crown of the tooth, the enamel separates from the dentine, sometimes as a complete cap, more often in pieces. In some cases pieces of the dentine adhere to the enamel. If this could not be readily separated, the whole piece was discarded.

This is much the most practicable and accurate method of obtaining enamel free from dentine of which I am aware. The objection to it is that it might influence the result of the density. This may or may not be so, but is not at present of great importance, since the conditions were the same for all the teeth, and it is the comparative density which is being chiefly considered. I have cut sections of enamel so treated, and examined them very carefully under the microscope, and am unable to make out any change of structure at all.

That a real difference does exist between the specific gravity 
and mass density of enamel is proved by the following test: The enamel of a sclerotic tooth was split off in the furnace $\left(300^{\circ} \mathrm{C}\right.$. for three minutes), and divided into two portions. The specific gravity of the first portion, estimated by the water method - taking the precaution to boil the enamel and soak it in water for several hours after weighing in air, and before weighing in water-was $3 \cdot 0$; whilst the density of the second portion, estimated by the pycnometer and mercury method, was 2.98 - that is to say, proportionately a smaller bulk of water was displaced than mercury. Or, expressed in another way, $3 \cdot 0$ represents the average specific gravity of the individual prisms, but 2.98 represents the density of the enamel as a whole plus enclosed air (most of the moisture or traces of organic material having been driven out wholly or partly by the previous heating).

In all, sixty-four teeth were examined by this method. The number might perhaps have been more with advantage, but it is extremely difficult to obtain a sufficient number of sound teeth of all classes. The process, moreover, to be undertaken with accuracy, involves a considerable expenditure of time. The temperature of the air near the pycnometer was taken regularly, and since it only varied slowly from $12^{\circ} \mathrm{C}$. to $15^{\circ} \mathrm{C}$., and since this would only affect the fourth decimal place, the results are not corrected for temperature. Throughout care was taken not to touch the pycnometer with the fingers, nor to allow the breath to influence its temperature.

The following are the average densities of the enamel of the three classes of teeth examined by this method:

\begin{tabular}{|c|c|c|c|c|c|c|}
\hline \multicolumn{5}{|c|}{ Teeth. } & $\begin{array}{l}\text { Density of } \\
\text { Enamel.* }\end{array}$ & Decrease. \\
\hline " Native' teeth & . & $\ldots$ & . & $\ldots$ & $2 \cdot 85^{8}$ & - \\
\hline "Sclerotic" teeth & . & .. & . . & . & $2 \cdot 821$ & $0 \cdot 037$ \\
\hline "Malacotic" teeth & . . & .. & .. & $\ldots$ & $2 \cdot 723$ & $0 \cdot 097$ \\
\hline
\end{tabular}

$*$ Water $=\mathrm{I}$. 
Thus it is seen that there is a maximum difference between the densities of "native" and malacotic teeth of O.I35-a not inconsiderable amount. It will also be observed that there is nearly twice as much difference between malacotic and sclerotic teeth as between the latter and " native " teeth. The lowest density observed was $2 \cdot 63$, and the highest $3 \cdot 06$. The teeth were selected and classed empirically from a nakedeye examination alone, and were otherwise selected indiscriminately as regards the sort of tooth-i.e., whether incisor or molar.

The average difference between the density of the three classes of teeth may, perhaps, not be very great; but having regard to the fact that the difference is probably due to the difference in porosity of the outer layers of enamel only, it would not be anticipated that the variation would be any greater. It is quite possible that some really sclerotic teeth were included amongst the "malacotic." It is quite easy to be deceived in this matter by the naked eye, since one usually associates a darkened yellowish colour with a sclerotic tooth, and if it has not this colour it is apt to be classed as a malacotic; whereas this by no means follows, some sclerotic teeth retaining their original lightness of colour.

It will be remembered that the permeability of enamel was found to decrease with the time after its formation. Therefore, if density is dependent upon this permeability (or porosity), we should expect to find a similar variation in density, though in the opposite direction. This, as a matter of fact, is found to be so. Teeth were taken from the jaws of a young Maori (about sixteen), and the density of the enamel estimated by the above method. The results are given in Table on p. I24.

It is seen that the density is least in the incomplete and unerupted tooth, and greatest in the tooth which has been erupted for the longest time; that the greatest increase in 
density occurs during the later stages of enamel formation; that the least increase occurs during the eruptive stage. And seeing that the density of the enamel of the first molar is greater than that of the second molar, the logical inference is that the density goes on increasing after eruption. The only other explanation is that all the teeth had originally and permanently different densities. This, however, I do not think at all probable, from my experience in examining the density of other Maori teeth. But the result of these estimations,

Tooth and Condition.

Third molar, unerupted, enamel incomplete. . Third molar, unerupted, enamel complete .. Third molar, partially erupted Second molar, erupted, say, three years First molar, erupted, say, nine years
Density of Enamel.

$2 \cdot 465$

$2 \cdot 75$ I

$2 \cdot 755$

$2 \cdot 780$

$2 \cdot 85 \mathrm{I}$
Increase

$0 \cdot 286$

$\mathrm{O} \cdot 0 \mathrm{OO} 4$

$\mathrm{O} \cdot 025$

$0 \cdot 07$ I

taken in conjunction with the permeability experiments, makes any such possibility even more remote. I think, therefore, as the result of these two investigations, that we may assume that the density of healthy enamel bears a direct ratio to the time which has elapsed since its formation.

\section{Hardness of the Enamel Surface.}

The methods which have been adopted in the past for the estimation of the comparative hardness of enamel do not seem to be of the right character. Resistance to crushing strain has been the principal scientific method used, but this, I think, does not meet the case. It cannot be regarded as a measure of true " hardness." For instance, the diamond and carborundum are both harder than iron, but the latter will stand far more crushing stress than either of the former.

The geological method of measuring hardness is far more exact and more applicable to the present case. In geology the hardness of minerals is measured by two methods : 
I. By scratching the surface with other minerals, thus placing them in a certain order, the one which is scratched by everything else being placed lowest, and the one which nothing else will scratch being placed highest.

2. By means of a special instrument-the " sclerometer."

The latter method seemed the more exact for the purpose of recording the hardness of the surface of enamel, and it was therefore adopted. Fig. 62 shows the form of sclerometer which was used for the purpose.

It will be seen that the tooth to be examined is made to

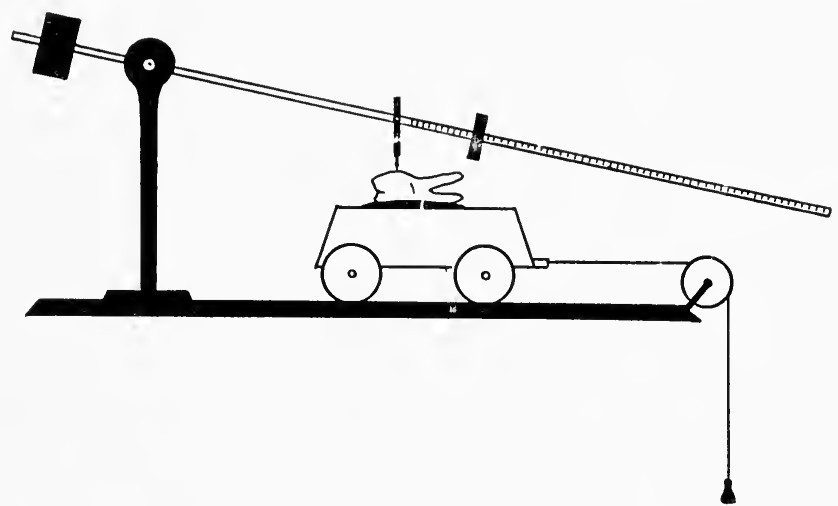

Fig. 62.-Diagram of the Form of Instrument USEd to indicate the Variations in H ARd NESS OF THE ENAMEL SuRface.

glide under a diamond point, which is attached to an accurately balanced brass rod. The rod beyond the point is graduated in centimetres and millimetres, and on the rod slides a 5 or Io gramme weight. By altering the position of the weight on the rod, an increasing amount of pressure can be made on the diamond point; and, since the weight is known and the distance is known, it is a simple matter to calculate the actual pressure upon the point at any moment. The method adopted was to draw the tooth under the point with gradually increasing pressures until a scratch was made in the enamel 
which could be distinctly seen with the naked eye when rubbed with graphite. This method, of course, has its liabilities to error, since it depends upon " what can be seen by the naked eye." This may vary with the observer and with the light. But in this case the observer was the same and the eyesight quite normal, and the light as far as possible always the same, in that all the observations were made before the same window and at the same time of day.

The difficulties of the method, however, are far more serious than these possibilities of error. If a perfectly smooth surface could always be obtained the matter would be simpler; but the varying curvature of the surfaces of the teeth, and the relatively marked or feeble development of the imbrication lines, make accurate observation in many cases somewhat difficult.

Another variation is introduced by the sharpness of the diamond point. Not that it wears or becomes smooth, but it may be accidentally broken, and the next point may not be of exactly the same shape or sharpness. So that only those teeth which are examined with the same point can be compared.

The method is somewhat tedious, and only sixty-five teeth of various kinds were thus examined; but these results, I think, sufficiently indicate the comparative hardness of the various classes. The average weight required to scratch the enamel was as follows:

\section{Point No. I (VERY SHaRp).}

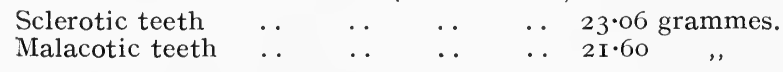

Point No. 2 (BLunt).

$\begin{array}{llllll}\text { Sclerotic teeth } & \ldots & \ldots & \ldots & \ldots & \text { I } 50 \text { grammes. } \\ \text { Malacotic teeth } & \ldots & \ldots & \ldots & \ldots & \text { roo }\end{array}$

Point No. 3 (medium).

Sclerotic teeth $\quad \ldots \quad \ldots \quad \ldots \quad \ldots \quad \ldots 33^{\circ}$ o grammes

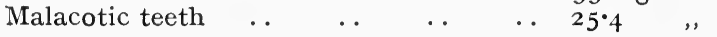


I think there is no doubt that these results will be confirmed by further investigation by this method, and also by the method of scratching with different minerals. At present the indications seem to be fairly constant, and support our contention that there is a difference between " hard" and "soft" teeth; that this difference is capable of being measured; and that the terms "sclerotic" and "malacotic" in their literal meaning are justified.

\section{The Comparatiye Solubility of Enamel of Various Types.}

That all enamel is soluble in acid, provided the acid is sufficient in amount or acts for a sufficient length of time, has long been recognized, and also that some acids, strength for strength, have greater solvent powers than others. In this respect the researches of Head are very interesting. Head* has shown that the solubility of enamel in various strengths of acid varies in a very curious manner, and often that an acid of extreme weakness causes more destruction of the enamel than a strong solution of the same acid. For instance, he states that a I in 20,000 acid sodium-phosphate solution caused obvious decalcification, whilst a I in 2,00o solution had no effect, and also that the total effect produced by the I in 20,000 solution was greater than that produced by a $\mathrm{I}$ in 20 solution. Head, however, does not state the total amount of acid salt present in each solution, and this I regard as of much more importance than the degree of dilution, as will be seen by subsequent experiments; neither does he state the type of tooth used in the various tests. Head also draws attention to the fact that whilst an acid of stated strength in watery solution produces decalcification, yet in a salivary solution it produces no effect. In this respect Head's work cannot be regarded as of much value, since apparently he did not estimate the total acidity (?) of the saliva after adding the acid. Obviously the

* Dental Cosmos, 1907, p. 8ox. 
acid in the saliva solution is largely, if not entirely, neutralized by the alkaline salts present, and also by combining with the protein constituents of the saliva, and the surface of the enamel would be further protected by the insoluble precipitate of albuminate thus deposited.

With a view to ascertaining whether enamel of various types differed in its solubility in similar acids of the same strengths, the following experiments were undertaken:

\section{Qualitative Estimation of Solubility in Lactic Acid.}

The periods of immersion in the acid were short, the object being to compare the relative rapidity with which each variety of enamel was attacked. An indication of the amount of solution or partial solution which had taken place was obtained by scraping (or cutting) the surface of the enamel with a sharp scalpel, so that the softened enamel remained on the blade. The amounts of white powder appearing on the blade were compared, and recorded as,,++++++ , the single + standing for a just perceptible amount of softening.

I. Three malacotic teeth were suspended in lactic acid I in 20,000 (0.05 c.c. in I,000 c.c. distilled water). In twelve hours there was no observable effect; in two days all showed dull surfaces, and a white film could be just scraped off with a sharp scalpel. At the end of three days an appreciable amount could be scraped off the surface of all the teeth, and under the microscope the surface showed disintegration. The decalcified film, however, could be removed from the surface of the enamel, leaving a "polished" surface* underneath.

2. Malacotic, sclerotic (European), and Maori molars were placed in a similar solution (lactic acid I in 20,000). At the end of two days the surfaces of the malacotic and sclerotic

* This "polish" (under the microscope), however, is made up of innumerable scratches running in all directions, all true surface "structure " being lost. 
teeth appeared dull, the Maori retaining its polish. With a sharp scalpel the surfaces of the malacotic and sclerotic enamel could be scraped very slightly, the former more than the latter, the Maori not at all. At the end of three days the malacotic and sclerotic teeth were quite dull to the naked eye, with a definitely formed film, and the Maori still retained a polish. The enamel of all the teeth could be removed from the surface with the scalpel: the malacotic +++ , sclerotic ++ , and the Maori + .

3. Malacotic, sclerotic, and Maori canine teeth were placed in lactic acid of varying strengths, the same amount of acid being employed in each case. In this and the following experiments canine teeth were used exclusively, in order that the conditions might be as uniform as possible, and because these teeth are recognized clinically as being more resistant to caries. All the teeth were perfectly sound. The amount of solution of enamel at the end of twelve hours may be tabulated thus:

\begin{tabular}{l|l|l} 
A. B. & C.
\end{tabular}

Variety of Touth.

\begin{tabular}{c|c|c} 
Lactic Acid & Lactic Acid & Lacti Acid \\
I in rooo. & $\begin{array}{c}\text { in } 500 . \\
\text { in roo. }\end{array}$ \\
0.05 c.c. in 50 c.c. & $0^{\circ}$ c5 c.c. in 25 c.c. & 0.05 c.c. in 5 c.c.
\end{tabular}

\begin{tabular}{|c|c|c|c|c|c|}
\hline Malacotic & . & . & $+t+$ & $++t$ & $++t$ \\
\hline Sclerotic . & . & . & $+r$ & ++ & ++ \\
\hline Maori $\quad$. & . & $\ldots$ & + & + & + \\
\hline
\end{tabular}

At the end of two days the relative amount of softening of each of the varieties in each of the solutions was the same, though of course it had advanced a little in all. After this period very little further softening took place--i.e., all the available acid had combined with the lime salts of the enamel.

The amount of softening of the various enamels occurring 
in the three solutions $\mathrm{A}, \mathrm{B}$, and $\mathrm{C}$, was most in $\mathrm{C}$, less in $\mathrm{B}$, and least in $\mathrm{A}$, but the difference was not very marked-nothing like so marked, in fact, as the differences betreen the various enamels $i_{n}$ each solution. (These results tend to show that the total amount of acid present is the important factor, and not so much its degree of dilution, the only difference being that in the extremely weak solutions a little longer time may be required. For instance, even with such an extremely weak solution as 0.05 c.c. in $\mathrm{I}, 000$ c.c., the result was almost the same, at the end of three days, as that of the 0.05 c.c. in 5 c.c. in one day.)

In all cases surfaces which had been subject to attrition were attacked before unworn surfaces. But in the above records, since the malacotic teeth showed no attrition, only unworn surfaces are compared.

\section{Qualitative Solubility in Orange-Juice.}

Four crucial incisions were made in a Tahiti orange, and the following teeth inserted: Maori canine, sclerotic canine, malacotic canine (complete-i.e., having been in use for some years), malacotic canine (root incomplete). At the end of a week the teeth were removed and dried.

The Maori Canine showed a questionable opacity in one small area, the remainder of the surface showing the normal lustre.

The Sclerotic Canine was opaque and friable where a small fracture had occurred at the mesial incisive edge; otherwise it was unaffected.

The Malacotic Canine (complete) showed a slight opacity along the incisive edge, but the opacity was not friable.

The Malacotic Canine (incomplete) showed opacities down the centre of both lingual and labial surfaces, and also at the tip of the cusp. The opacities were all friable. 
The teeth were replaced in the same orange, but in fresh incisions, and examined at the end of the second week.

The Maori Canine showed no increase of the original opacity, but a fresh and friable opacity had appeared on the edge of an attrition area; otherwise the enamel surface was highly polished and hard.

The Sclerotic Canine: The original opacity had become more friable, no further opacities had appeared, and the surface was otherwise normal.

The Malacotic Canine (complete) : The original opacities along the incisive edge were more marked, and an opaque area had appeared on the mesial surface. There was no loss of substance, and the opacities were not friable.

The Malacotic Canine (incomplete) showed increased opacity of the original areas, and also loss of substance along the opaque line on the labial surface.

The teeth were replaced, and examined at the end of the third week.

The Maori Canine showed no fresh opacity, and, moreover, the original opacity seemed somewhat less marked.

The Sclerotic Canine showed an increased friableness of the original area at the incisive edge, but was otherwise unaffected.

The Malacotic Canine (complete): The original opacities had increased in intensity and become friable at the cusp. Two fresh opaque lines had appeared on the lingual and labial surfaces.

The Malacotic Canine (incomplete): The whole of the enamel surface had lost its lustre, and the greater part of the lingual and labial surfaces were opaque. There was loss of substance on both these surfaces, and the cusp was very friable. 


\section{Quantitative Estimation of Solubility in Hydrochloric Acid.}

Teeth of the following types were selected: Maori, sclerotic, malacotic, and malacotic unerupted. The whole of each tooth was covered with wax, and then two areas (each $4 \mathrm{~mm}$. square) of the enamel surface of each was exposed. Each tooth was then carefully weighed. They were then placed in I per cent. hydrochloric acid for forty-eight hours. At the end of this time they were removed, and washed in running water for twelve hours in order to remove the disintegrated enamel. They were then dried and weighed. Fourteen teeth were thus examined. They all, as would be expected from the action of such a powerful acid as hydrochloric, showed considerable loss in weight, but this loss again varied according to the "type" of tooth. The following are the average losses in the four classes:

\begin{tabular}{|c|c|c|c|c|c|c|}
\hline \multicolumn{4}{|c|}{ Class of Tooth. } & & \multicolumn{2}{|c|}{ Loss of Weight. } \\
\hline Маові & .. & . & & & O•OI 4 & mme, \\
\hline Sclerotic & $\ldots$ & . & . & $\cdots$ & 0.018 & , \\
\hline Malacotic & . & . . & . & . & $0 \cdot 028$ & , \\
\hline Malacotic & (unerupted) & $\ldots$ & $\ldots$ & $\ldots$ & $0 \cdot 050$ & ,. \\
\hline
\end{tabular}

Thus it is seen that the unerupted malacotic teeth lost nearly twice as much as the erupted malacotic, and the latter lost half as much again as the sclerotic; whilst the sclerotic lost one and two-sevenths as much as the Maori, and the malacotic enamel had lost exactly twice as much as the Maori.

The results of these experiments on the solubility of enamel go to show that teeth do vary in their resistance to acids-to lactic, citric, and hydrochloric acids, at least. The results are remarkably uniform for all three acids, and show throughout that the enamel of malacotic teeth is most soluble, and that of the " native" teeth least soluble in all cases. Here, also, the empirical and clinical classification is justified by experimental investigation. 
The fact that the enamel of unerupted teeth was nearly twice as soluble as that of erupted teeth is very significant, and corresponds in this respect closely to the results of the "permeability" experiments. In fact, the results of these "solubility" experiments correspond so closely with those on the permeability of enamel that the inference cannot be avoided that the variations in solubility of the various classes of enamel is due to the variation in permeability.

\section{The Effect of Carbon Dioxide in Solution.}

It has been suggested that possibly the presence of an abnormal amount of carbon dioxide in the saliva might have a decalcifying effect upon the enamel. Miller produced decalcification in enamel sections by the action of a stream of water containing $\mathrm{CO}_{2}$ in solution. Recently J. M. Lewis of Ielbourne has confirmed this. But, as I have pointed out, such observations do not bear upon the present issue, since it is with the natural enamel surface that we are so intimately concerned, and this behaves towards acids very differently from any artificially formed surface. Drs. Head and Waller both suggest that the surface of enamel may be dissolved by $\mathrm{CO}_{2}$. My observations do not support these suggestions. My disbelief is based upon the following experiments. Analyses of saliva* give the total amount of carbon dioxide present as varying from about 40 to 60 volumes per cent. The greater part of this, however, is a chemical combination, and only a little over 3 volumes per cent. are in solution.

I. A solution was therefore made up containing 60 volumes of carbon dioxide in 100 volumes of water (the actual amount being I litre). Maori, sclerotic, and malacotic canine teeth (two of each) were placed in the solution for one month without the slightest effect being produced on any of them.

* Külz, quoted by B. Moore, Schäfer's " Textbook of Physiologv," p. 346 ; and by Halliburton, "Physiological Chemistry," p. 626 
2. Two thousand c.c. of carbon dioxide were then dissolved under pressure in 500 c.c. of water, and a number of teeth kept in this solution for two months. At the end of this time neither softening nor opacity of the enamel had occurred. The only effect produced was that the calculus present on some of the teeth had become quite soft and crumbling.

3. A 30 per cent. solution of carbon dioxide was made in water. (The water was very slightly allialine, but the addition of the $\mathrm{CO}_{2}$ gave a marked acid reaction with litmus.) This solution was made to flow over malacotic, sclerotic, and Maori teeth for two months, the loss of $\mathrm{CO}_{2}$ by evaporation being constantly made good. No effect whatever was produced on the enamel surfaces of any of the teeth except one, and this was a malacotic tooth with a carious cavity which had been filled with wax. The solution had evidently got under the wax and produced fresh decalcification of the already carious enamel.

4. Carbon dioxide was bubbled slowly through saliva containing malacotic, sclerotic, and Maori teeth for two months. The result was exactly similar to the above. No effect was produced on the enamel surfaces of any of the teeth except one, and that one a malacotic tooth previously carious.

I do not therefore think it is at all likely that, even by a much more prolonged action, the comparatively small amount of free $\mathrm{CO}_{2}$ present in saliva could possibly be regarded as a factor in the initiation of caries.

It is possible, however, that it is not without action on damaged surfaces and in carious cavities.*

* Head suggests measuring the amount of surface decalcification (?) by an instrument which he has devised for measuring resistance to pressure. Waller suggests that the increase in calcium in the water should be estimated. I think both these methods are open to serious liabilities to error, and are not so reliable as an examination of the surface under the microscope. 


\section{Hypothesis as to the Cause of Permeability and the Function of Nasmyth's Membrane.}

Sufficient evidence has, I think, been adduced to show that enamel is permeable, and that the permeability decreases in certain cases after the eruption of the teeth. It remains now to consider by what means this might possibly be brought about. It is obvious that when enamel is first secreted it must be in a semi-fluid condition, and that a subsequent hardening process must take place. This hardening or vitrifying process cannot be an extremely rapid one, and the evidence goes to show that this hardening process is not quite complete in the last-formed enamel when the tooth erupts. It is probable that the stellate reticulum has something to do with the abstraction of the lime solvent, and that it gets crowded out of existence, as it must do finally, before its function is quite complete.

In considering the cause of the imbrication of the enamel surface, it was suggested as probable that an increased pressure on the ameloblasts in small jaws gave rise to the increased development of imbriæ found on "malacotic" teeth. Subsequently it has been shown that the surface of malacotic teeth is also more permeable, even at the time of eruption, than native teeth. The probability is that the two phenomena are due to the same cause- a too hasty completion of the enamel surface owing to pressure on the enamel organ caused by a too small crypt.

The enamel after eruption of the tooth is covered by a dead membrane-Nasmyth's membrane; it is immersed in a fluid saliva, containing in solution lime salts and organic material (mucin and albumin), and it has been shown that fluid with solid in solution can pass into the outer layers of enamel. It would seem, therefore, in every way highly probable that Nasmyth's membrane acts as an ordinary 
dialyzing membrane, through which crystalloids pass, but colloids do not. Therefore, other things being equal, and so long as the lime salts, especially the calcium phosphate, remain in solution, they must tend to pass through the membrane and penetrate the enamel, and the mucin and albumin are kept back. No doubt the process is very slow and gradual, depending largely upon the relative osmotic pressures on either side of the membrane, but it must undoubtedly take place.

Now, the calcium phosphate is held in solution in the saliva by the presence of carbon dioxide, and this tends sooner or later to be given off. If it is given off early, then the phosphates are precipitated, and cannot, of course, pass through the membrane. If it is not given off so soon, the phosphates will pass through the membrane in solution; but here sooner or later the $\mathrm{CO}_{2}$ must be given off and pass back again, and the phosphates be precipitated. Now, if, as was shown to be probable, traces of the " enamel solvent " remain in the outer strata of enamel, this must be in a form of albumin-probably lymph charged with $\mathrm{CO}_{2}$; but the $\mathrm{CO}_{2}$ would be lost soon after eruption, and so the fluid come to consist of lymph alone. The phosphates therefore would be precipitated in an albuminous medium, and would form calcoglobulin, the peculiar indestructible nature of which is well known. Thus the minute interstices in the enamel would eventually be filled up and protected by a highly resistant substance. The rate at which this would take place is obviously dependent upon the condition of the saliva. If the saliva is scanty in amount, or if conditions are present which tend to precipitate the phosphates and other lime salts, then the process will be retarded or entirely prevented. Similarly, an excess of viscous mucin in the saliva will be deleterious, since this substance is a non-electrolyte, and it is a well-known fact that the presence of non-electrolytes in solution with electrolyte salts considerably hinders, and may, indeed, entirely prevent, dialysis 
from taking place. This supports the clinical association between viscous saliva, malacotic teeth, and extensive caries often observed in children.

Again, acids have a much lower "endosmotic equivalent" than alkaline salts; and so, when enamel is first erupted and in a permeable condition, if lactic acid is formed on its surface, it will tend to pass through Nasmyth's membrane by dialysis at a greater rate than will the alkaline salts of the saliva, and thus give rise to that increased opacity of typical malacotic teeth which precedes their general destruction. This would also explain the considerably lessened effect of lactic acid on sclerotic teeth which have become less permeable; since, owing to the occlusion of the porosities, no dialysis would be possible, and hence what solution did occur would be quite superficial, and therefore much slower.

This hypothesis would explain, too, the staining of the enamel so constantly associated with sclerotic teeth. It is well known that such stains cannot be removed, and it is difficult to account for them unless it be on the assumption that coloured fluids or salts of the saliva have passed into the outer strata of the enamel and there become fixed.

The further consideration of the subject is so intimately associated with variations in the composition of the saliva that it must be deferred until the latter has been dealt with.

\section{The Possibility of rehardening Enamel.}

Head* has stated that enamel, after partial decalcification (in acid calcium phosphate, weak lactic acid, and orangejuice), and subsequent prolonged immersion in saliva, not only loses the opacity due to the decalcification, but also becomes quite hard again. If this can be substantiated, and such hardening effected at will, it is obviously a most impor-

* Dental Cosmos, January, r9ro, p. $4^{6}$. 
tant matter, and one which might have very far-reaching effects in the treatment of initial caries.

I have repeated Head's experiments, and carried out others on similar lines. Teeth-native, sclerotic, and malacotichave been immersed in various strengths of lactic acid and of orange-juice, and exposed to the fermentation of carbohydrates with saliva, until well-marked opacities of the enamel were formed and the surface could be cut with a sharp scalpel. They were then transferred to saliva, and kept in it (with occasional changes of saliva) for a period of three months. The reaction of the saliva was always alkaline. At the end of this period the teeth were removed and washed in running water, dried carefully with filter-paper (friction being avoided), and exposed to the air for four hours. On examination, in one tooth-a malacotic canine-the enamel, which was previously soft and friable, was certainly considerably harder, and the opacity had almost disappeared. In another tooth--a Maori canine-a very small and rather doubtful opacity had also disappeared. In all the remainder (seventeen) the opaqueness of the enamel was still present, and had not apparently decreased, although it seemed that in some cases the enamel had become slightly harder and required more pressure to cut it with the scalpel than it did originally.

The results of these experiments, then, neither confirm nor negative Dr. Head's hypothesis. They will require repeating several times before a definite conclusion can be formed. I think it is not unlikely that it will be found that under certain conditions rehardening both can and does occur. The conditions may relate to the enamel-the manner and stage of its decalcification-or to the amount and character of the saliva. This requires very careful determination.

There is one fallacy, however, which it is necessary to guard against. Very weak lactic acid produces an even opaque film all over the teeth immersed in it. This decalcified film may be removed, leaving a smooth, polished, and hard 
surface underneath. If, after being kept in saliva, this film be accidentally removed in the process of drying, it may be thought that the enamel has "rehardened." Such a mistake may be quite easily obviated by examination of the surface under the microscope. P. rtial decalcification up to the point of opacity does not destroy the imbrication lines nor the outlines and central depressions of the enamel prisms. If, however, the film of partial decalcification has been removed, all trace of normal structure will likewise have disappeared.

In the decalcification produced by the fermentation of carbohydrate, the opacity is of a different nature. The surface often remains highly glazed, but the acid penetrates more deeply and irregularly into the enamel, and a rough, irregular surface remains behind after removal of the partially decalcified portion. So that it would seem to be indicated that, in further work on this subject, only enamel decalcified by exposure to lactic-acid fermentation should be used.*

\section{Conclusions.}

I. That the enamel of teeth varies appreciably as to surface structure, hardness, density, permeability, and solubility.

2. That these variations are measurable, and in all cases bear a distinct relationship to the clinical classification of teeth into sclerotic and malacotic types-that, in fact, there is a demonstrable difference between "hard" and "soft" enamel, and that these differences are sufficiently constant to justify the terms.

3. That this difference is not wholly developmental, but is partly acquired.

4. The difference in surface structure is, of course, wholly developmental. A nearer approach to the " native" type,

* Further experiments, however, have shown that the difference is probably brought about by the modifying influence of the saliva, since a mixture of lactic acid and saliva, with the former in excess, produces a similar kind of decalcification to that caused by the fermention of carbohydrates. 
and therefore a more highly resistant enamel, is to be attained by a more physiological use of the jaws in very early infant life, this continuing especially during the time that the roots of the deciduous molars are developing, in order to stimulate both the growth of the jaws and the full development of the tooth germ and crypt.

5. The difference in density and permeability are probably partly acquired, and are dependent on the dialysis of lime salts from the saliva. The details of means whereby the saliva may be so modified or increased as to facilitate such dialysis are considered later.

6. Finally, in order to obtain enamel of the highest resistance to caries, it is necessary to promote those conditions, hygienic, dietetic, and prophylactic, which will insure-

(a) The normal development of the enamel organ to its fullest extent.

(b) That the phosphates and other lime salts shall remain in solution in the saliva for as long a time as possible.

These conclusions are in the main largely supported by clinical experience. Many older practitioners who have treated families for a considerable number of years are of the opinion that teeth, if they are not destroyed by caries, do become " harder " after eruption. These opinions have been received, however, with scepticism in the past. But are we right in entirely discarding such observations simply because they are not supported by the readings of a scientific instrument? Are not the eyes, hands, and brains, of men long trained to their special work and fitted by years of observation, scientific instruments of extreme delicacy? True, the personal equation is a factor to be reckoned with, but when the average of a large number of such observations is all in one direction we cannot do otherwise than pay considerable attention to the conclusions. 


\section{CHEMICAL COMPOSITION OF ENAMEL}

IT is not proposed here to deal with the minute analysis of enamel, but merely to give several facts which relate to its density and resistance. Von Bibra gives the percentage of organic substances as 3.59 per cent., and the inorganic salts as $96.4 \mathrm{I}$ per cent. Hoppe-Seyler's analysis gave 3.6 per cent. organic matter. Tomes* was able to prove that enamel (elephant's) contained practically no organic matter, and that probably water of crystallization has been included under this head in previous analyses.

No comparative analyses of human enamel from persons of different ages have been made, so far as I am aware, except that Hoppe-Seyler states that "infantile" enamel (enamel of unerupted teeth) contains more organic matter. This, however, is an important indication, and will be referred to later. Recently, an endeavour has been made to show that those teeth which contained most organic matter were more resistant to caries.

In the Zeitschrift für Physiologische Chemie for Igo8, Band iv., p. 455, is published a paper by Gassmann, in which are recorded his results of the analysis of human and dog's teeth. The figures are given on the following page.

The loss on incineration represents, of course, water of crystallization and organic matter. It will be seen from these figures that apparently there is more organic matter in human

* Journal of Physiology, 1896. 
canine teeth and dog's teeth than in human wisdom-teeth. Yet, as Gassmann points out, the latter are more prone to caries, and this would seem to infer that the more organic

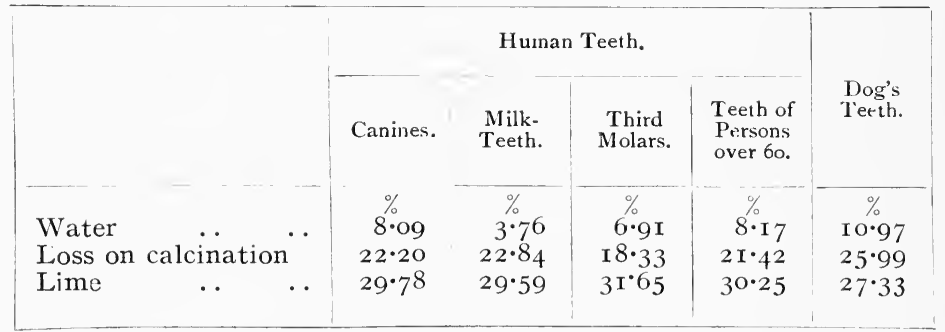

matter a tooth contained, the better the tooth would be. Herein lies a serious fallacy, for it does not appear that the author has taken into account the varying proportions of enamel in the various kinds of teeth analyzed. For instance, if a tooth had a large crown and a small root the percentage of lime salts would be high, and the organic matter relatively low, since the percentage of enamel would be high; and the converse would be true if the tooth had a small crown and a long root, where the proportion of enamel would be small. And it is just in these respects, unfortunately, that the two classes of teeth-canines and third molars-vary so greatly.

Comparatively small amounts of tissue were analyzed, so that it cannot be claimed that an average was struck. Moreover, in the dog's teeth especially, if not in the others, wear and tear considerably diminishes the amount of enamel present, and thus the total percentage of lime salts in the tooth would be much diminished. And anyone who cuts sections of dog's teeth will be surprised at the comparatively small amount of enamel remaining in adult animals.

It is not at all clear that an analysis of entire teeth can yield results of any value bearing upon the question of immunity and susceptibility to caries. The enamel alone is so essentially the tissue of resistance that the composition of dentine and 
cement does not seem to enter into the question at all-at least, until it has been definitely proved that these latter substances vary in composition in direct proportion to the variation in the enamel of the same teeth.

\section{The Calcium-Magnesium Balance.}

Röse* has shown by an extended series of investigations that a relationship exists between the hardness of the water in any locality and the incidence of caries. He found that the condition of the teeth was related to the quantity of calcium salts in the drinking-water, but not to the quantity in farm products. He also states that the important factor is not so much the initial hardness as the stable hardness of the water --that is, the amounts of calcium and magnesium sulphates in solution which are not precipitated upon boiling.

The most perfectly built teeth were found in provinces where the water, besides calcium, contained magnesium salts in the proportion of $4: \mathrm{I}$. The character of the teeth, too, found in districts rich in mineral salts correspond to what we have described as sclerotic teeth, whilst those from districts poor in mineral salts were malacotic in type.

The artificial addition of salts of calcium and magnesium to water has so far not been so successful as to warrant its adoption as a therapeutic measure, and Röse recommends the prescription of natural waters rich in mineral salts. He seems to ascribe the beneficial result of hard waters partly to more perfect development of the teeth, and partly to a resulting increase in the alkaline salts of the saliva. The effect on saliva of giving calcium salts internally has recently been investigated by J. M. Lewis (University of Melbourne). He finds that neither the alkalinity nor the phosphatic indices of the saliva are thereby appreciably affected.

Now, Bunge has proved that when the intake of potassium

* Deutsche Monatsschrift f. Zahnheilkunde, January-June, I9o8. 
salts exceeds that of sodium there is an increased excretion of sodium salts; and Malcolm,* working by analogy, has shown that a similar relationship exists between calcium and magnesium. It has been shown that the ingestion of soluble magnesium salts causes a loss of calcium in adult animals, and hinders its deposition in young growing animals, while soluble calcium salts do not in the same way affect the excretion of magnesium.

Weiske's $\uparrow$ experiments also support these findings. For instance, of two rabbits, one received I gramme $\mathrm{CaCO}_{3}$ daily in addition to its food, the other I gramme of $\mathrm{MgCO}_{3}$, for three months. The rabbits were then killed, and it was found that, although they were of equal body-weight, the total weight of the bones (dried and fat-free) in the first rabbit exceeded that of the second rabbit ( $77 \cdot 45$ grammes : 69.52 grammes); and, further, that the amount of organic matter in the bones of the $\mathrm{MgCO}_{3}$ rabbit was in excess of that in the $\mathrm{CaCO}_{3}$ rabbit.

This would seem to be in opposition to Röse's view, that the addition of magnesium increased the resistance of the teeth to caries. Dr. Malcolm and the author therefore undertook an investigation to determine whether the ingestion of magnesium salts had a similarly deleterious effect upon the teeth. Four rabbits of the same age (six weeks) were selected: two were given ordinary food, and acted as controls; the other two were given in addition magnesium chloride in solution. The amount given was at first I c.c. of $0 \cdot 25$ per cent. $\mathrm{MgCl}_{2}$ once daily. This was gradually increased up to 0.5 gramme $\mathrm{MgCl}_{2}$. At the end of three months the rabbits were killed.

Microscopically there was no difference observable between the teeth of the two sets of rabbits. Sections were made of the incisor and first molar teeth of each rabbit. A microscopical examination of these revealed a number of minute

* Journa of Physiology, February 28, 1905, p. I83.

$\uparrow$ Quoted by Malcolm, loc. cit. 
differences, but nothing which could be regarded as constant or as distinctly abnormal. For instance, granularity of the enamel prisms and interglobular spaces in the dentine were found to a slight extent in both series, but it could not be determined whether more in one than another.

The density of equal portions of similar teeth was determined by the pycnometer and mercury method, the result being slightly in favour of the controls:

$$
\begin{array}{cccccc} 
& & & & \text { Controls. } & \mathrm{MgCl}_{2} \text { Rabbits. } \\
\text { Average density } & \ldots & \ldots & \ldots & \mathrm{I} \cdot 924 & \mathrm{I} \cdot 885
\end{array}
$$

Difference $=0 \cdot 039$.

The hardness of the enamel of the incisor teeth, estimated with the sclerometer, showed the enamel of the "normal" rabbits to be slightly more resistant than that of the magnesium rabbits. Similar teeth were taken from the jaws of

\begin{tabular}{|c|c|c|c|c|}
\hline & $\begin{array}{l}\text { Weight of } \\
\text { Dried Teeth. }\end{array}$ & $\begin{array}{l}\text { Percentage } \\
\text { of } A \text { sh. }\end{array}$ & $\begin{array}{l}\text { Percentage } \\
\text { of Organic } \\
\text { Material. }\end{array}$ & $\begin{array}{l}\text { Percentag, } \\
\text { of Calcium" } \\
\text { in Ash.* }\end{array}$ \\
\hline $\begin{array}{l}\text { Average for controls } \\
\text { Average for } \mathrm{MgCl}_{2} \text { rabbits }\end{array}$ & $\begin{array}{l}I \cdot 28 I 9 \\
I \cdot 2797\end{array}$ & $\begin{array}{l}77 \cdot 7 \\
76 \cdot 8\end{array}$ & $\begin{array}{l}22 \cdot 25 \\
23 \cdot 20\end{array}$ & $\begin{array}{l}36 \cdot 8 \\
36 \cdot 4\end{array}$ \\
\hline
\end{tabular}
each rabbit and analyzed, with the following results:

The amount of difference is only slight. Clearly the teeth are not affected to the same extent as bones, though they are slightly affected and in a similar manner. Thus the magnesium salts evidently had the effect of lowering the percentage of inorganic and raising the percentage of organic material in the teeth; also the average percentage of calcium is a little lessened.

Of course, whole teeth were analyzed here, too, though in this case, since similar teeth were taken, in each instance

* In the first edition of this book, by a curious oversight, the percentage of calcium was stated to be one-half of what it really should have been. 
the relative amount of enamel was the same. There is no means of knowing, however; whether the differences existed proportionately more in the dentine than in the enamel:

The results, then, of this investigation do not, on the whole, bear out Röse."s wiews as a magnesium salts being particularly desirable for producing dense dental tissues. It would seem, perhaps, that the calcium salts are, after all, the more important.

Röse's investigations are inconclusive in another respect, in that a large number of concomitant circumstances bearing upon the habits and diet of the inhabitants of the various districts are not taken sufficiently into consideration, and may be such as to entirely negative any effect produced by the water. To quote only one instance, the geological formation of South Wales consists largely of calcareous and carboniferous rocks. The waters derived from these have a faintly alkaline reaction, and the mineral solids consist chiefly of sulphate and carbonate of calcium and magnesium.* Yet the incidence and extent of caries amongst the population is enormously high.

On the other hand, the geological formation of the Scotch Highlands is largely granitic and Devonian. The water from such sources is often faintly acid in reaction, and contains only traces of lime and magnesia. $\uparrow$ Yet the excellence of the Scotch Highlanders' teeth is proverbial.

We conclude therefore that, although the hardness of the water may be a factor in the production of a relative immunity to caries, it is by no means so important as to be an essential factor.

* Parkes and Kenwood: "Hygiene and Public Health."

$\dagger$ Ibid. 
THE FORCES OF NATURAL PROTECTION

II. ACTIVE-THE ORAL SECRETIONS 



\section{CHAPTER XII}

\section{T H E S A L I V A}

For many years now it has been recognized that the quantity and composition of saliva has some effect upon the teeth. As to what exactly the effect is, however, there is a considerable divergence of opinion. So wide, indeed, is the divergence that, whilst some observers ascribe immunity to caries to salivary protection, others ascribe the occurrence of caries to the deleterious effects of salivary constituents.

It is perfectly obvious that a fluid in which the teeth are permanently bathed, which flows over the teeth to the possible extent of several pints daily, cannot be without effect upon the teeth. Again, since this is a normal physiological condition, it cannot be but that it is a beneficial one, and one which we should be justified in stating to be protective in function even though no more were known about it. Protection against disease is afforded to nearly all tissues largely by the fluid in which they are bathed, and the only fluid capable of so protecting the surface of the teeth is the saliva. It is, too, a general pathological law, and not confined to the human tissues, but pervading the whole of organized nature, that stagnation in any tissue or cavity makes for decay and disease, and favours the development of those general analytical forces represented as a rule by the activity of micro-organisms; and, conversely, all those forces which prevent stagnation and promote movement and circulation make for health and development. If the salivary secretion did naught else, it 
would be bound to act in such a manner by promoting a circulation in the mouth, and preventing oral stagnation.

Cases in which a diminution of the salivary flow is so marked as to be noticeable, and perhaps distressing, are occasionally recorded, and these are almost invariably accompanied by acute and rapidly spreading caries of the teeth. Such cases are recorded by Miller, * Rigolet, $\uparrow$ and Dubreuil-Chambardel. $\ddagger$

Indeed, when we remember that the greater proportion of human beings exist on fermentable carbohyhrates-and we have seen that even the teeth of races most immune to caries are attacked in vitro by the acids of fermentation--we recognize that there must exist some potent form of protection, or there would be no such thing as immunity to caries; and, in fact, it is extremely likely that in the process of evolution teeth would long ago have succumbed.

The importance of saliva in this respect-as the fluid which is largely responsible for the health of the buccal cavity and its contents--has not received the attention it merits. Attention has been so concentrated upon two of its componentsptyalin and mucin-and their function in the digestion of starch and swallowing of food respectively, that nearly all else has been regarded as subsidiary. Pavlov, in his classical lectures on "The Work of the Digestive Glands," remarks that " the fluid must be of great importance as a cleansing agency," yet this is " hardly taken into account in physiology,"' Even Pavlov, however, only thinks of the mucous membrane, and does not mention the teeth.

\section{Conditions of Normal Secretion.}

It is a most important fact to recognize, though one which appears to have been almost entirely overlooked by workers

* Dental Cosmos, 1903, p. 694.

‡ British Dental Journal, 1908, p. 88o.

$\S$ "The Work of the Digestive Glands," second edition, p. 69. 
in the subject from a dental point of view, that the normal secretion of saliva is a reflex one; that it is called forth in response to stimuli applied to various parts of the mouth; and further, and most important of all, this reflex mechanism is, as we shall show, a most delicate one, but nevertheless a most certain and reliable one. It is one having far-reaching effects, and is capable of being either stimulated or depressed, and of bringing about very considerable differences in the composition of the saliva.

The afferent side of the reflex arc is represented by the lingual and glosso-pharyngeal nerves in the tongue, and by their specialized terminations in the organs of taste; and also to a lesser extent by the sensory branches of the fifth nerve, supplying the buccal mucous membrane and teeth. It is the function of these nerves under normal conditions, upon being stimulated, besides making the act of mastication pleasant, to cause a secretion of saliva which is exactly adapted to the stimulus which called it forth. That is to say, the chief physiological use of the sense of taste is to control the activity of the salivary glands. It used to be thought that the excitation of the nerves of taste had a considerable effect on the gastric secretion; but Pavlov,* in his work on these glands, has now come to the conclusion, and brings forth very convincing experimental evidence in support of the view, that the excitation of the sensory buccal and lingual nerves has no action whatever in directly stimulating gastric secretion.

If this view be correct-and it appears to be supported by Starling $\uparrow$ - the association between the gustatory nerves and the salivary glands becomes all the closer. It becomes more clear that the raison d'être of the nerves of taste is to act as sentinels in the mouth, on whose warning a flow of saliva appears, which completely envelops any substance in the

* “ The Work of the Digestive Glands," second edition, p. 9r.

$\dagger$ " Recent Advances in the Physiology of Digestion." 
mouth, neutralizing and counteracting any harmful effects, and preparing the food for deglutition, or enabling it to be expectorated if disagreeable, in either case facilitating its rapid removal from the mouth, and preventing its adherence to the buccal tissues (including the teeth). Thus we may say that the function of the afferent buccal and lingual nerves is very largely protective, and their extreme importance, therefore, from a dental point of view-as being the originators of the means of automatic protection of the teeth-is at once evident.

The efferent side of the arc is represented by the chorda tympani nerve in the submaxillary and sublingual glands, and by the auriculo-temporal nerve in the parotid gland. Fibres of sympathetic nerves are also supplied to each gland, and these also transmit efferent impulses.

The reflex path, then, is thus: The sensory nerves of the tongue are stimulated by some sapid substance; the impulse passes up to the centre in the medulla, whence it is reflected down special fibres of the chorda tympani or the auriculotemporal nerve, and, arriving at the gland, causes an immediate secretion of saliva.

It is upon the integrity, excitability, and " education" of this reflex arc that the protection and freedom from disease of the teeth very largely depends.

\section{Psychical Secretion.}

A copious flow of saliva of varying composition can be induced reflexly by means of stimuli conveyed via the optic, olfactory, or auditory nerves. This applies especially if the subject be hungry. That is to say, the sight or smell of certain articles of food, or the sound of its preparation (or even the dinner-gong), may cause, as is well known, the "mouth to water."

The secretion may be modified considerably by other impressions, visual and mental, received at the same time, 
pleasurable stimuli increasing the secretion, and disagreeable sensations depressing the activity, of the glands. It is extremely doubtful, though, whether psychical secretion would occur if it were not for previous education of the gustatory nerves-i.e., through certain sounds or sights having become reflexly associated by long experience with a pleasurable stimulation of the lingual and glosso-pharyngeal nerves.

Sellheim* has performed experiments which show that after section of both of these nerves the secretion of parotid saliva in the dog remained as copious as before when substances were placed in the mouth; but it does not appear that the psychic effect had been eliminated, and that the secretion was not due to what might be termed a " memory reflex."

The study of the salivary secretion has been until comparatively recently confined almost entirely to conditions in which the efferent side of the arc was alone stimulated, the usual method of obtaining saliva for observation and analysis being by electrical stimulation of the chorda tympani nerve. This has at least two disadvantages:

I. The stimulus is not a natural one; it is in a form which is untranslatable, and we do not know to what it corresponds normally.

2. It stimulates all the fibres in the nerve at one and the same time, and we have reason to believe that there are several different sets of fibres (or at least two), each having a specific effect upon salivary secretion, and excited by different reflex stimuli from the mouth.

So that, having obtained a result by this method, we do not know under what conditions a similar result (as to quantity and composition of saliva) is obtained by the natural stimulation of the afferent buccal and lingual nerves.

A similar objection may be made to the method of those who have worked at the subject from a dental point of view

* Quoted by Pavlov, ibid., p. 82. 
alone, and have collected the saliva from the human subject during dental operations. The saliva so obtained cannot be relied upon as being in any degree normal, since the stimulus is essentially an abnormal, and perhaps a very painful, one, and a similar flow might be obtained, perhaps, by stimulating the sciatic nerve. Neither can chewing a piece of cotton-wool (as advocated by Miller) be regarded as being a natural means of exciting salivary secretion. The saliva so obtained does not represent any ordinary state of the oral secretions for that individual, since it is obtained under quite abnormal conditions.

Sellheim* adopted the method of obtaining salivary secretion in the dog by means of food material and chemical substances placed in the mouth. There is, however, at least one serious objection to Sellheim's results from chemical substances-the latter were all used in too strong a solution to be comparable with ordinary dietetic effects. For instance, saccharıne Io per cent. was used-a strength which would in the human being at least produce intense nausea. Even o.oI per cent. tastes very sweet indeed. The same objection applies to sodium carbonate Io per cent. and sodium chloride 20 per cent. Similarly, hydrochloric acid 0.5 per cent., sulphuric acid 0.6 per cent., and formalin 0.5 per cent., are all far in excess of what could be normally tolerated.

In fact, such results can only be taken as exemplifying the "rinsing" effect of saliva (so called by Pavlov)--the reflex secretion that occurs in order to rid the mouth of obnoxious or harmful substances (and which is analogous to the reflex flow incited by certain disagreeable odours). It is a form of stimulation which under voluntary conditions occurs very rarely, and therefore the saliva so obtained must be regarded as being of very rare composition also.

Again, too, an objection exists that salivary secretion in

* Quoted by Pavlov, ibid., p. 72. 
the dog is produced under normal conditions by very different substances from those affecting human beings. A point which seems to have escaped observation in this connection is the shape, disposition, and function, of the teeth. A dog's teeth are not adapted for mastication of material like white bread, and he therefore spends much more time and muscular activity in eating a piece of bread than he would a piece of meat of similar size; the bread bothers him, and is not natural. Whereas in the human being precisely the reverse obtains: the habit is to masticate meat carefully, because the teeth are flattopped and not carnassial in type, and the tendency is to slightly squeeze food like bread between the teeth and to swallow it without proper mastication at all; and lack of mastication means decreased salivary secretion.

In order, then, to avoid these objections, the following conditions should be observed:

I. The saliva should be obtained from the human subject.

2. It should be obtained by natural or ordinary methods of stimulation, or, if in the "resting stage," under known and constant conditions.

3. The subject should be one in whom (in order to get " average" results) there is apparently an average salivary activity.

In order that these conditions might be observed, the following series of investigations have been carried out. So far as I am aware, the effects of ordinary dietetic stimuli on the separate and combined salivary secretions in man have not been previously determined, the reason probably being the difficulty of collecting the saliva from the several glands during mastication. Langley* states: "In man the data are not sufficient to form an estimate of any value either of the relative amount of saliva secreted from the various glands or of the total amount secreted in twenty-four hours." The first

* Schäfer's "Textbook of Physiology," p. 49r. 
thing, then, obviously, was to devise an apparatus by which this might be done. For some time the difficulties were insuperable, but eventually the following means were adopted: For the parotid saliva a cannula was used in Stenson's duct. The ordinary cannulas or any small tubing obtainable were all either too large or made of too thick metal, so that the lumen, in proportion to the diameter of the cannula, was always too small. Cannulas were therefore made of thin German silver of as large a size as could possibly be inserted into the duct (the size being determined by first passing a graduated bougie, and marking the limit of its insertion). By bending the cannula slightly, it was found that it could be held in the duct* without much discomfort during the mastication of any substances.

For the submaxillary and sublingual glands a small apparatus that might be termed a "segregator" was made. This is an appliance made of vulcanite, something like an artificial denture, but having a hollow chamber attached at one side (made of soft vulcanized rubber). It is open at the bottom, and the margins are exactly applied to the floor of the mouth, so as to include the orifices of the submaxillary and sublingual glands. A metal tube (3 millimetres in diameter) has its orifice inside the chamber, and as nearly as possible over the opening of the ducts. The tube passes up through the vulcanite, through a space between the teeth, and then forwards in the buccal sulcus, where a small rubber tube is attached, which passes out of the mouth to the required receptacle. Fig. 63 represents the segregator diagrammatically.

This apparatus was found to work well, and to give (within reasonable limits) very constant results. It does not, obviously, separate the sublingual from the submaxillary secretion, but these are probably very similar, so that it is not a matter of great importance. The saliva is secreted at con-

* Securing it to the moustache was found to be a good way. 
siderable pressure, so that it easily forces its way up the tube and round the bend; no aspiration is necessary, and it is not desirable.

The apparatus requires to be made, of course, from a plaster cast of the floor of the mouth of the subject of the observations.

The investigations have been divided into several groups,
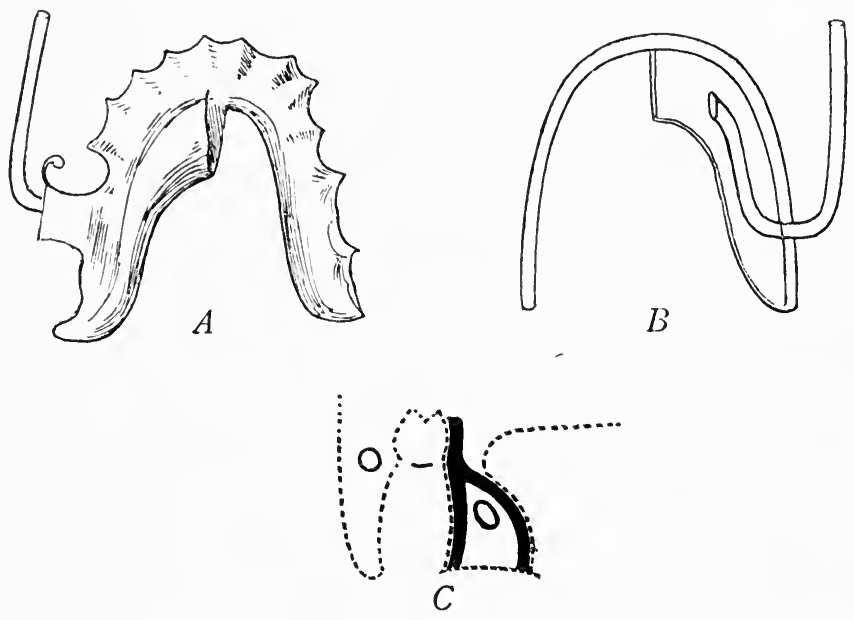

Fig. 63.- $A$, Upper Surface of Segregator; $B$, Under SurfaceDiagrammatic; $C$, Diagrammatic Section of Segregator in Position.

according to the particular constituent of the saliva under observation.

Having regard to the previous conclusion that probably the chief function of the saliva is protective, it follows that probably, also, all its constituents are of importance from a protective point of view. I do not think it will be of any value to state here the composition of saliva as ordinarily given in textbooks, since we do not know the exact conditions under which it was obtained. The chief constituents from a dental protective aspect are-water, the alkaline salts of 
calcium and sodium, the phosphates of calcium and mag.nesium, ptyalin, sulphocyanate of potassium, sodium chloride, and mucin.

Detailed observations have been made under varying conditions on all these constituents, and will shortly be described. In the first place, however, it is necessary to call attention to a most important point. The mere estimation of the proportionate amounts of the constituents, or the "percentage composition," is of no great value by itself. It would be so if the mouth were a sealed cavity and always contained the same amount of saliva, but it is not and does not. It is like a testtube with a hole in the bottom and with a reagent flowing in at varying rates. Obviously, then, the other important thing is to know the rate of flow per minute, and to calculate the total amount of substance per mimute present in the mouth.

For instance, analysis may show 5 per cent. of a substance to be present; but if only I c.c. of saliva flows per minute, it will not be anything like so efficacious, because there will not be so much of it, as if it contained only 2 per cent. but the flow were 5 c.c. per minute.

It is the ratio, then, of the amount per c.c. to rate per minute which is all-important. In the subsequent estimations this total amount or value of any substance in the mouth per minute has been termed an " index "-i.e., the " alkalinity index," the " phosphatic index," the " ptyalin index," etc.

It is evident, too, that the rate of flow must be extremely important in considering the mechanical cleansing of the mouth and teeth by the water of the saliva alone, and the dilution of the acids formed by fermentation. The greater the flow per minute, the more rapidly will the acids be carried away. It is always a question of time. The organisms form a certain amount of acid from carbohydrate in a certain time; and it is a question whether the saliva can dissolve and carry away the carbohydrate débris or neutralize those acids as they 
are formed, or whether the acid production gets ahead of the saliva, and so is free to combine with the lime salts of the enamel.

\section{The Amount and Alkalinity.}

For the following estimations saliva was collected with the apparatus described, during the mastication of various substances used ordinarily as food. The amounts were measured to 0.05 c.c., and the alkalinity estimated by titration with $\frac{\mathrm{N}}{50} \mathrm{H}_{2} \mathrm{SO}_{4}$, this extreme dilution being necessary to insure anything like accuracy. The titration was done immediately after collection. The indicator used was weak methyl orange. Other indicators are quite unsuitable; litmus cannot be used, because it is affected by carbon dioxide, which is always present, and almost certainly in varying quantity and in varying conditions. If the saliva be boiled to drive it off, some of the alkaline salts are precipitated, and this may interfere with the titration. Moreover, if acid phosphates happen to be present, these may turn the litmus either red or blue.

Phenolphthaleïn also is affected by $\mathrm{CO}_{2}$, and cannot be used in the presence of ammonia, which is sometimes present in saliva, and it is therefore unsuitable.

The alkalinity is expressed in c.c. of $\frac{\mathrm{N}}{50} \mathrm{H}_{2} \mathrm{SO}_{4}$ required to bring the methyl orange to neutral point. It has been left as such on account of simplicity and the readiness with which differences may be recognized in reading a table of the results.

The experiments were all done (with one or two exceptions) at the same time of day and under as similar conditions as possible as regards previous meals, exercise, occupation, and temperature of atmosphere. In the majority of cases the estimations have been repeated at least twice, and sometimes three times, where any uncertainty existed; and a number of early estimations of possible faulty technique have been discarded altogether.

In order to have some basis of comparison between " rest- 
ing" saliva-i.e., saliva present in the mouth some hours after eating - and saliva during mastication, the amount and alkalinity of specimens obtained by expectoration was estimated on fifty consecutive days (excepting Saturdays and Sundays), two hours after a light midday meal, which always consisted of the same things. The average of these estimations is referred to as " normal resting" saliva. Fifteen minutes after collection of the saliva during mastication, the "resting " saliva was also estimated, in order to gain an indication as to the possible prolonged effect of the stimulus.

Table I. gives the results of this series of estimations. It is seen that the amount of saliva per minute varies very much both from the parotid and from the submaxillary and sublingual glands; that it may under some conditions from the parotid be ten times as much as from other stimuli; that from the submaxillary it may be eight times as much from one stimulus as from another; that the total amount of saliva per minute in the mouth likewise may vary in the same proportion -i.e., as 8 : I.

A very noticeable point is the comparative smallness of the parotid secretion per minute. It never amounts to onethird of that from the submaxillary and sublingual glands, and may be as low as one-twentieth or one-thirtieth,* the average amounts being as $I: 5.89$ for this series of stimulations. It seems not at all unlikely that this small quantity of parotid secretion is the most important factor in producing the increased susceptibility of upper teeth to caries (see p. I7). As regards the relationship of stimuli to amount per minute, it is quite obvious that the most sapid substances produce the greatest flow, and of these sapid substances the ones which are most acid are the most powerful.

The softest and least flavoured articles produce the smallest

* Smale and Colyer state that two-thirds are secreted by the parotid glands ("Diseases and Injuries of Teeth," p. $48 \mathrm{r}$ ). 


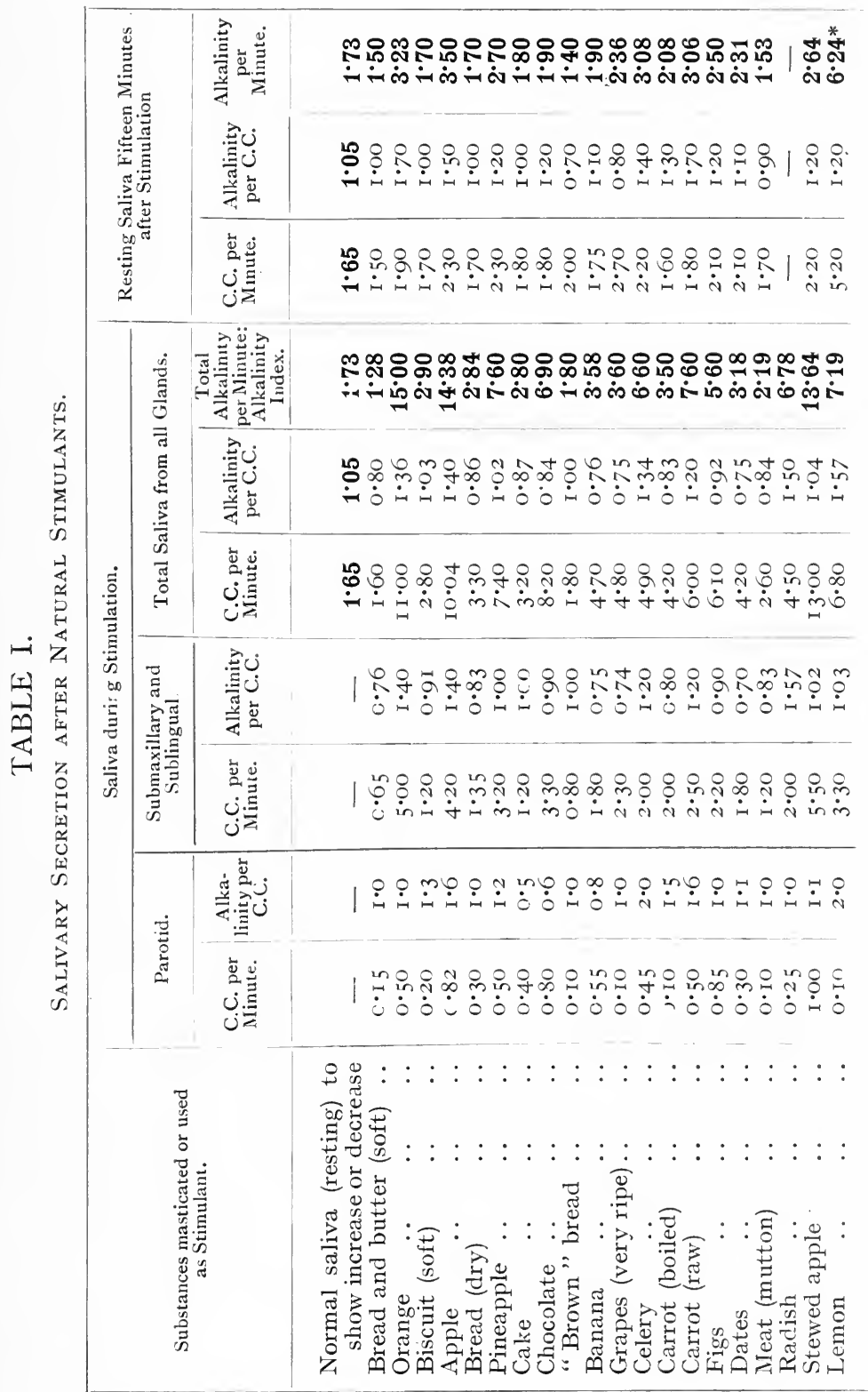


secretion. Compare the amounts from each of the glands and the total from all glands for the following substances: Buttered bread and orange, biscuit and apple, cake and celery, etc. Sweet substances, like chocolate, dates, and figs, also produce a fair amount of secretion, but not so much as the acid ones. Pavlov found that dry substances in the dog produce the greatest parotid secretion. It is seen here, however, that, whilst dryness does increase parotid secretion in man ( $2: \mathrm{I})$ - $c f$. bread and butter (soft) and dry bread-it does not do so to anything like the same extent as acid substances, like soft stewed apple, for instance $(6: \mathrm{I})$. All the substances except one produce an increase in the total amount over the resting saliva, in the case of acid substances very considerable, and of sweet substances very marked; but in the case of the more tasteless articles the increase is very much less. Dry bread (white), doubles the amount; brown bread (soft) only barely increases it by O.I5 c.c. per minute; orange and apple increase it by more than six times. Bread and butter actually depresses the secretion by 0.05 c.c. per minute. This, I think, is due to the demulcent effect of the butter. It prevents the oral mucous membrane from being stimulated, becomes fluid in the mouth, and possibly prevents any slight stimulus from the bread reaching the taste-buds, by coating the tongue or the particles of bread with an oily neutral fluid.

The alkalinity of the saliva produced by the various stimulants shows similar, though not parallel, variations to the amount. The amount may be increased, but not the alkalinity per c.c.; or the alkalinity per c.c. may be increased, but not the amount; or both may be increased or decreased in varying proportions, according to the substance being masticated. The average alkalinity of the parotid saliva per c.c. is greater than that from the other glands; but, owing to the lesser flow per minute from the former gland, the total alkalinity per minute is very much less. 
Here again is seen the fact that the most acid substances produce the greatest alkalinity per c.c. Sweet substances markedly depress the alkalinity per c.c. (cf. chocolate, cake, grapes, banana, figs, and dates), but this deficiency may be in some cases more than made up for by the greater total quantity per minute. Although the variations in the alkalinity per c.c. are exceedingly interesting as showing the extreme delicacy of the mechanism controlling salivary secretion, and that a fluid exactly adapted to the stimulant is always forthcoming, yet it is the total alkalinity in the mouth per minute which is the most important point.

If we refer now to the column giving this (the first column of heavy figures), it is made quite clear that acid substances produce an overwhelming alkalinity per minute as compared with such substances as bread, biscuit, cake, etc.-that, in fact, the alkalinity produced by acid substances may be to that produced by neutral ones as II : I.

The question, though, naturally arises, Is the alkalinity so produced only sufficient to neutralize the acid substance in the mouth so that a mixture of the two would be just neutral ? From observations made on this point, it would appear that the saliva is usually able to neutralize all substances (except intensely acid ones) during mastication; but this point is not so important as the resulting reaction of the saliva and oral secretions some little time after the food material has been eaten-that is to say, does the stimulation or depression of the salivary glands cease with the swallowing of the stimulant, or is it continued for some time afterwards? Is there a sufficient "afterflow" to cleanse the mouth and neutralize and alkalinize any products of fermentation? Obviously, from a prevention-of-caries point of view this is of prime importance. The columns in the various tables under "Resting Saliva Fifteen Minutes after Stimulation " provide the answers.

It is apparent that the stimulation and depression is pro- 
longed for at least fifteen minutes (and there is reason to believe for considerably longer). In only two instances is there an actual fall below the amount of "normal resting" saliva-in the case of bread and butter and boiled carrot.

The alkalinity per c.c. shows a falling off in many instances -bread and butter, bread (white and brown), cake, biscuit, and meat. The total alkalinity per minute--the " alkalinity index "'-is lowered also in several cases, chiefly by bread and butter, brown bread, meat, and biscuit. This, in view of the large proportion such articles form of our modern dietaries, is an exceedingly important and significant fact. They are substances (except meat) from which, as we shall show, organisms are capable of forming a very large amount of acid by lactic fermentation in the mouth, and yet they are the ones which are least neutralized and diluted by the saliva.

On the other hand, it is equally clear that, after other substances, the amount, the alkalinity per c.c., and the alkalinity per minute, are all markedly increased. Again, it is the acid substances which produce this effect ( $c f$. orange, apple, pineapple, celery, carrot, lemon).

The action of lemon requires some comment. During mastication, it evidently does not produce an amount of alkalinity equal to apple, for instance, although it is far more acid; but two minutes after the cessation of the stimulus a profuse flow, with an exceedingly high alkaline index, occurs. This is to be accounted for, I think, by the stimulus in the first place being too strong, and producing a paralyzing effect upon the afferent nerves.* When the acid has been diluted and neutralized by the saliva somewhat, the full effect of the stimulus is felt, resulting in an alkalinity index three and a half times above normal.

* This explains the phenomenon that people who suck lemons continuously-i.e., Sicilians-show a wasting of the enamel surface. Nevertheless, recovery of the salivary glands must take place similarly, for, despite the lessened resist: $n$ ce of the teeth, caries is very rare. 
We are therefore justified in stating that the total amount of saliva poured out in response to acid articles of diet is more than enough to neutralize them, and is sufficient to exert a neutralizing and cleansing effect in the mouth for a considerable period afterwards.

It would therefore appear from the above (and subsequent) observations that it is extremely doubtful whether acid substances which have been masticated ever reach the gastric mucous membrane as acids. This, of course, does not apply to fluids, but even then, as is shown below (Table IV., p. I7I), the amount and alkalinity of saliva are increased to an extent which must make an acid reaction in the stomach of very short duration. It is therefore probable that when gastralgia follows the consumption of acid substances it is due to a salivary insufficiency.

\section{Variations in Secretion due to Taste Stimuli Alone.}

In the above series of estimations the substances have varied considerably in physical consistency, and have included practically only neutral, sweet, and acid flavours and their combinations. In order, therefore, to eliminate physical consistency, and to obtain results which should be due to the chemical nature alone, the following method was adopted:

Ordinary plain biscuits were selected as a "base," and by soaking them in various solutions, and then redrying them in an oven, the consistency remained the same; but each had its peculiar and distinctive "flavour."

The mere fact of adding water to the biscuits and then drying them produced a somewhat hardening effect, so that the control for this series must be a biscuit so treated, and not the normal untreated biscuit.

Table II. shows the solutions and the strengths in which they were used. The percentages of the solutions were such that they could be distinctly tasted, but not disagreeably so. The 


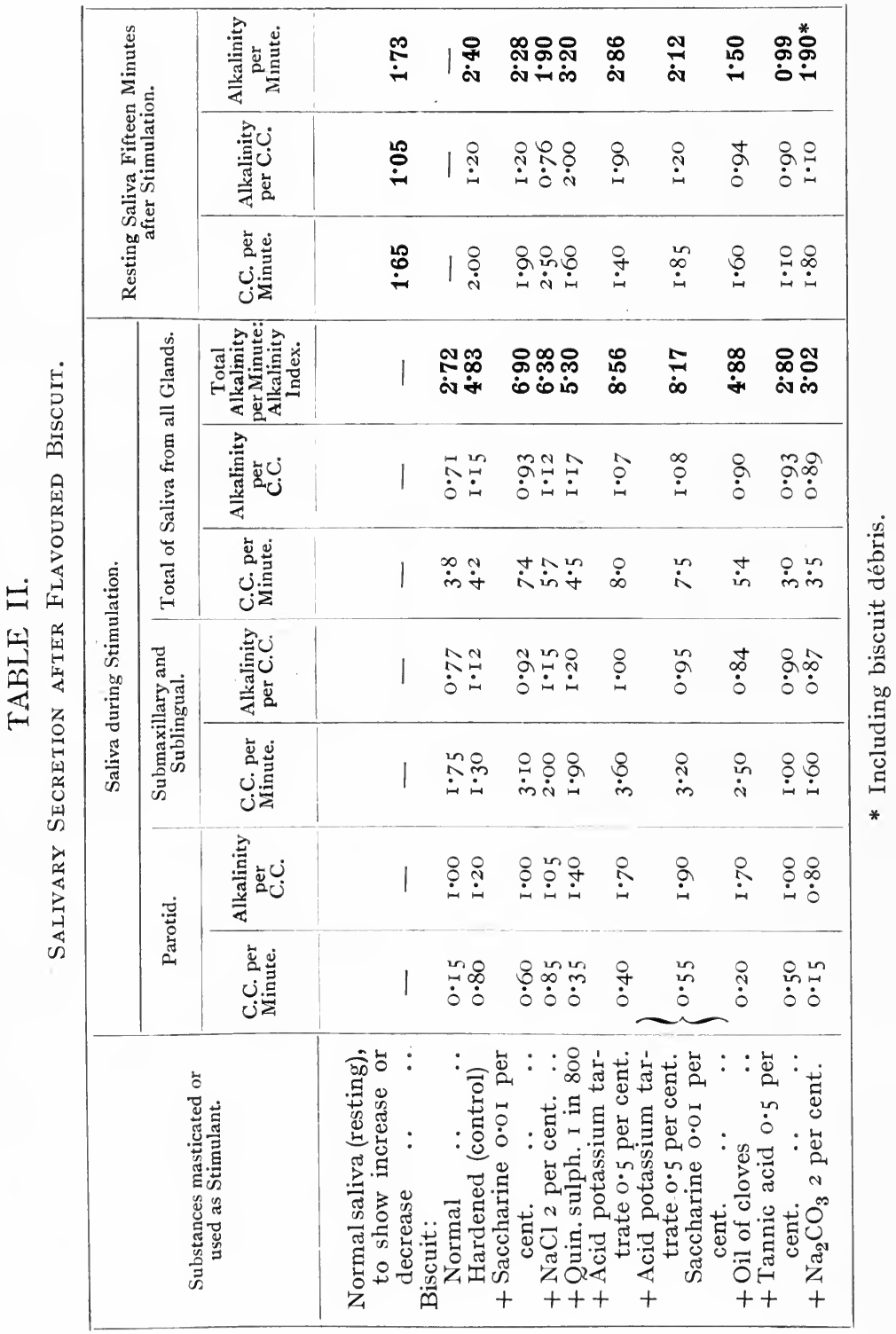


resulting effects on the amount and alkalinity of the saliva are tabulated as before. It is seen, firstly, that the mere fact of hardening the biscuit somewhat produces an increased flow and alkalinity from both glands, and also that the effect is prolonged.

The pure sweet taste of saccharine produced an increase in amount, but not in alkalinity, per c.c.-in fact, this is slightly decreased; but the total alkalinity per minute is very considerably increased, and also lasts for some time.

Salt (sodium chloride) and quinine sulphate also act as salivary stimulants in no small degree, increasing both amount and alkalinity. But the falling off in the alkalinity of the subsequent " resting saliva " is very marked in the case of the sodium chloride; probably, too, the amount falls considerably below the normal some time afterwards, for, as is well known, salt-flavoured articles produce an after "dryness of the mouth."* The prolonged action of the quinine sulphate is due, undoubtedly, to the fact that bitter flavours in the mouth are difficult to get rid of, and thus the afferent nerves are stimulated for a longer period than by others.

Again, it is clear that an acid flavour-acid tartrate of potash-produces the greatest secretion and the highest alkalinity index, this being nearly double that of the control during mastication. The increase is one of 5.84 over the "normal " biscuit, and 3.73 over the " hardened." So that we may say that an increase of $2 \cdot \mathrm{I}$ is due to hardening, and 3.7 to acidity. The combination of acid potassium tartrate and saccharine produces a very similar effect, though not quite so marked. Oil of cloves, as representing aromatic substances, produces very little increase in either amount or alkalinity. Its effect rapidly passed off, and, in fact, we get a subnormal index at the end of fifteen minutes.

* The question of the relationship of the physiology of "thirst" to dental caries is at present under consideration. 
Tannic acid, as might be anticipated, has a very inhibitory effect. The total amount during mastication is $I \cdot 2$ c.c., the alkalinity index $2 \cdot 0$ below the control, and the subsecuent " resting saliva" is very markedly subnormal in all respects. This also is a very significant fact when it is remembered how prevalent is the habit of drinking stewed tea combined with carbohydrates.

Sodium carbonate, representing weak alkalies, also has a markedly depressant effect. The total amount per minute is 0.7 below the control, the alkalinity per c.c. is 0.35 below, and the alkalinity per minute is $I \cdot 9^{8}$ below, during mastication. Afterwards it apparently does not sink quite to normal, but this is not really so, since a little biscuit débris (of course strongly alkaline) was contained in the saliva tested. The mouth was not washed out to remove this, since the mere fact of doing so may alter the subsequent amount of saliva.

The salivary glands are in some respects analogous to the pancreas, and Pavlov* has shown that the effect of alkaline salts upon the pancreatic secretion is inhilitory. He has further observed that in a dog which had an ossophageal fistula, and had had an addition of soda to its food for several weeks, the food which fell from the fistula was " hardly at all insalivated "; whereas in dogs fed without the soda the food was always copiously insalivated. This depression by alkalies is another very important point, which will be referred to again subsequently.

It has been shown, then, that acids are the stimulants which produce the greatest and most alkaline secretion of saliva. The question which next arises is, Are all acids alike in this respect?

In Table III. two inorganic acids are compared with two organic acids, the strengths of these solutions also being such as could be just comfortably tolerated.

* "The Work of the Digestive Glands," p. 232. 
TABLE III.

Salivary Secretion after Acids.

\begin{tabular}{|c|c|c|c|c|c|c|c|}
\hline \multirow{3}{*}{$\begin{array}{l}\text { Substances masticated } \\
\text { or used as Stimulant. }\end{array}$} & \multicolumn{7}{|c|}{ Saliva during Stimulation. } \\
\hline & \multicolumn{2}{|c|}{ Parotid. } & \multicolumn{2}{|c|}{$\begin{array}{l}\text { Submaxillary } \\
\text { and Sublingual. }\end{array}$} & \multicolumn{3}{|c|}{$\begin{array}{l}\text { Total of Saliva from } \\
\text { all glands. }\end{array}$} \\
\hline & 岂怤 & 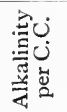 & 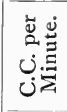 & 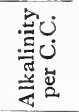 & 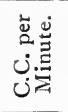 & 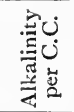 & 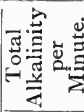 \\
\hline \multirow{5}{*}{$\begin{array}{l}\text { Normal saliva (rest- } \\
\text { ing) to show in- } \\
\text { crease or decrease } \\
\text { Biscuit }+\mathrm{H}_{2} \mathrm{SO}_{4} \mathrm{O} \cdot \mathrm{I} \\
\text { per cent. } \\
\text { Biscuit }+\mathrm{HCl} \quad \ldots \cdot \mathrm{I} \\
\text { per cent. } . \\
\text { Biscuit }+ \text { citric acid } \\
\text { I per cent. } \\
\text { Biscuit }+ \text { tartaric } \\
\text { acid I per cent. .. }\end{array}$} & - & - & 一 & 一 & $1 \cdot 65$ & $1 \cdot 05$ & $1 \cdot 73$ \\
\hline & O.I 5 & $\mathrm{I} \cdot \mathrm{OO}$ & $I \cdot 2$ & 0.58 & $2 \cdot 70$ & 0.62 & $1 \cdot 69$ \\
\hline & O.IO & $\mathrm{I} \cdot 75$ & $I \cdot 5$ & $0.7 \mathrm{I}$ & $3 \cdot 20$ & $0 \cdot 77$ & $2 \cdot 48$ \\
\hline & $0 \cdot 30$ & $I \cdot 30$ & $2 \cdot 7$ & $0 \cdot 90$ & $6 \cdot 00$ & 0.94 & $5 \cdot 64$ \\
\hline & & $\mathrm{I} \cdot \mathrm{OO}$ & $2 \cdot 0$ & I.30 & $5 \cdot 4^{\circ}$ & $\mathrm{I} \cdot 20$ & $6 \cdot 60$ \\
\hline
\end{tabular}

It is seen that the tartaric and citric acids give both a greater secretion from all the glands and also a higher alkalinity per c.c., and therefore a much higher alkalinity index than the hydrochloric and sulphuric acids. Both the hydrochloric and sulphuric biscuits, however, produced an astringent effect in the mouth, whilst the citric and tartaric produced a pleasurable sense of " succulency."

If these results, then, mean anything, they show that the afferent and efferent salivary mechanisms are indeed very delicate, and capable of appreciating very fine differences in stimulation, such as the different flavours of the acids, and of being depressed by some and increased by others. It is to be noted, too, that citric and tartaric acids (the latter especially) are acids widely distributed in Nature in fruits and vegetables, and therefore in a form accessible and suited for consumption. 


\section{The Effect of Liquids.}

Various liquids were drunk previously to eating a biscuit (normal and unhardened), to observe whether the amount and alkalinity of the saliva would be affected thereby. Table IV. gives the details of the results.

It is seen that no increase-in fact, a decrease-is produced by plain water, both in amount and alkalinity. Tea likewise causes a decrease in the alkalinity, but increases the amount, probably on account of the thein acting as a vasomotor stimulant; the amount, though, is not equal to what it should be (see Table II.), and the alkalinity index is subnormal.

Milk is only slightly better. Ale produces a marked increase in amount and alkalinity, due to the alcohol and the bitterness. The greatest increases, though, are produced by whisky and port wine. Whisky increases the amount, but the alkalinity per c.c. is decreased, also probably a vasomotor effect. Again, it is observed that the most acid liquid (i.e., port wine) has given rise to the highest alkalinity index.

The effect of water, tea, and port wine on the resting saliva fifteen minutes after drinking is given below. The depressant effect of the tea on the alkalinity is again marked.

Here, then, we have advanced a step farther, and see that the character and amount of the saliva during the mastication of one and the same substance may be very materially modified by the fluids previously taken.

\section{Acid Dietaries.}

The above conclusion having been reached, we are very naturally led on to the next step, the consideration of the effect of an acid diet on the saliva.

In order to determine this, an " acid " diet was substituted for the ordinary diet for a period of eleven days. During the first six days nothing was taken either to drink or to eat 


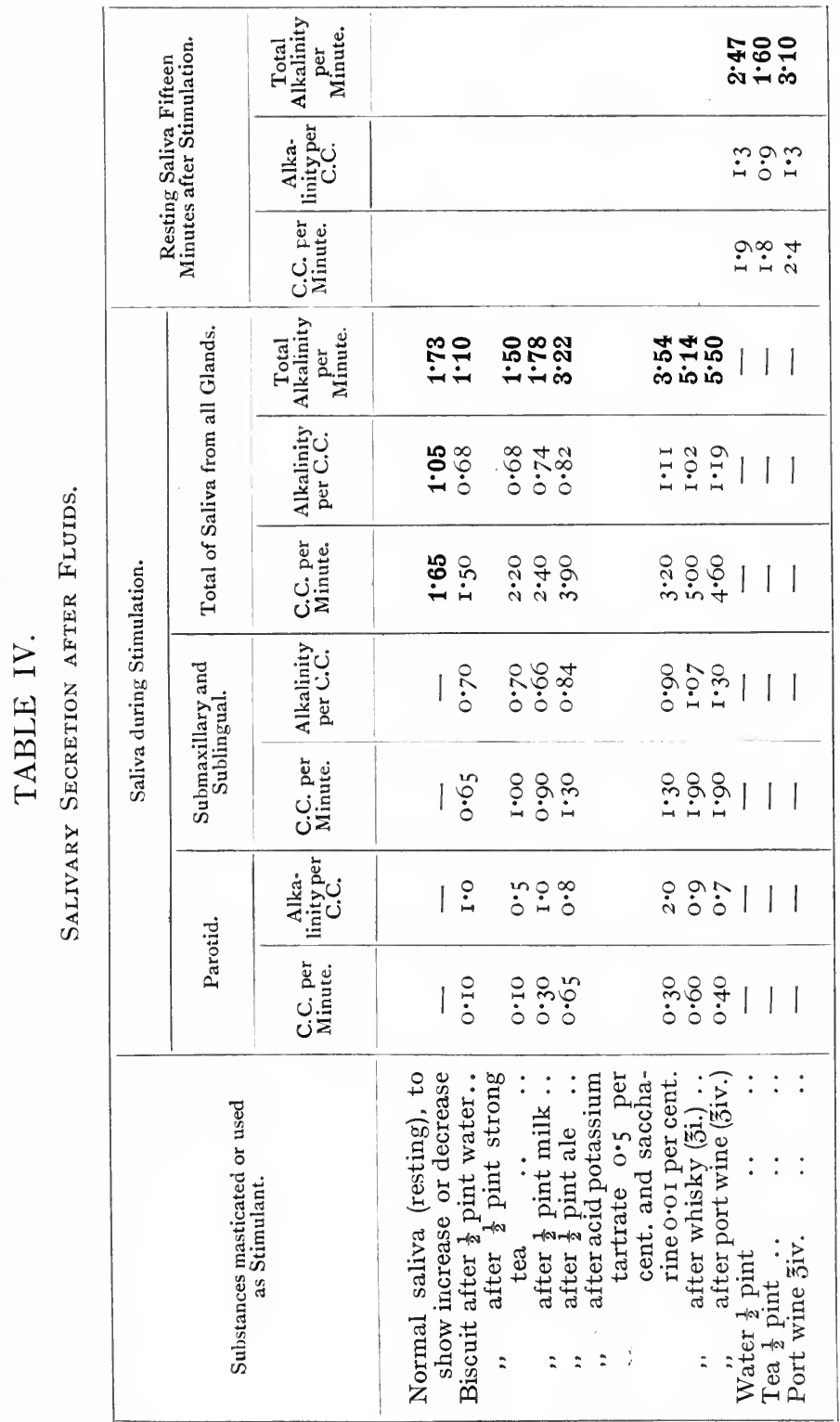


(except small quantities of milk and bread) which had not an acid reaction. Chiefly, therefore, it was a diet of fruit and vegetables. Meat was taken, but was rendered acid by sauces. During the last five days other substances were also taken, such as bread and butter, potatoes, milk puddings, etc.; but the meals always began and ended with " acids." The resting saliva was tested each day two hours after the

\section{TABLE V.}

Salivary Secretion DURING AN AcID Diet.

\begin{tabular}{|c|c|c|c|c|c|c|c|}
\hline & & & & & \multicolumn{3}{|c|}{ Total Saliva from all Glands. } \\
\hline & & & & & $\begin{array}{l}\text { C.C. } \\
\text { per Minute. }\end{array}$ & $\begin{array}{l}\text { Alkalinity } \\
\text { per C.C. }\end{array}$ & $\begin{array}{l}\text { Alkalinity } \\
\text { Index. }\end{array}$ \\
\hline \multirow{3}{*}{\multicolumn{5}{|c|}{$\begin{array}{l}\text { Normal resting saliva, to show increase. } \\
\text { Average resting saliva for first six days } \\
\text { Average resting saliva for last five days }\end{array}$}} & $I \cdot 65$ & $I \cdot I 5$ & $1 \cdot 73$ \\
\hline & & & & & $2 \cdot 50$ & $I \cdot I I$ & $2 \cdot 55$ \\
\hline & & & & & $2 \cdot 68$ & $I \cdot I 4$ & $3 \cdot 05$ \\
\hline \multicolumn{3}{|c|}{$\begin{array}{l}\text { Biscuit (previously) } \\
\quad \text { During full acid diet : }\end{array}$} & $\cdots$ & . & $3 \cdot 80$ & $0 \cdot 77$ & $2 \cdot 72$ \\
\hline Day 4 & . & . & . & . & $4 \cdot 50$ & 0.80 & $3 \cdot 60$ \\
\hline,$\quad 5$ & . & . & . & . & $4^{\circ} 90$ & $\mathrm{I} \cdot \mathrm{OO}$ & $4 \cdot 90$ \\
\hline Partial a & et: & $\cdots$ & $\cdots$ & . & $4 \cdot 80$ & $I \cdot I 0$ & $5 \cdot 60$ \\
\hline Day 7 & . & . & . & .. & $5 \cdot 20$ & $0 \cdot 70$ & $3 \cdot 64$ \\
\hline, 8 & . & . & & .. & $4 \cdot 90$ & $0 \cdot 75$ & $3 \cdot 70$ \\
\hline , 9 & . & . & . & . & $5 \cdot 20$ & 0.76 & $4 \cdot 00$ \\
\hline , IO & . & . & . & .. & $5 \cdot 20$ & $0 \cdot 80$ & $4 \cdot 20$ \\
\hline ,, II & . & . & . & . & $5 \cdot 80$ & $0 \cdot 83$ & $4 \cdot 81$ \\
\hline
\end{tabular}

midday lunch, and on eight of the days saliva was collected from the ducts during biscuit mastication.

In Table $\mathrm{V}$. and the chart (Fig. 64) the average results, without giving the details for each gland for each day, are shown. From a study of this table, it is at once evident that such a diet very materially increases both the amount and alkalinity of the saliva. The highest alkalinity index is during the second period for "resting" saliva, but during the first period for biscuit mastication. (This discrepancy 
between " resting " and "stimulated" saliva has been noticed on several occasions.)

It is interesting to note how the amount per minute and the alkalinity per c.c. increase gradually each day during each period. Between the sixth day and the seventh day of acid diet a day intervened on which ordinary diet was taken. The effect of this is evident in the marked falling off of the alka-

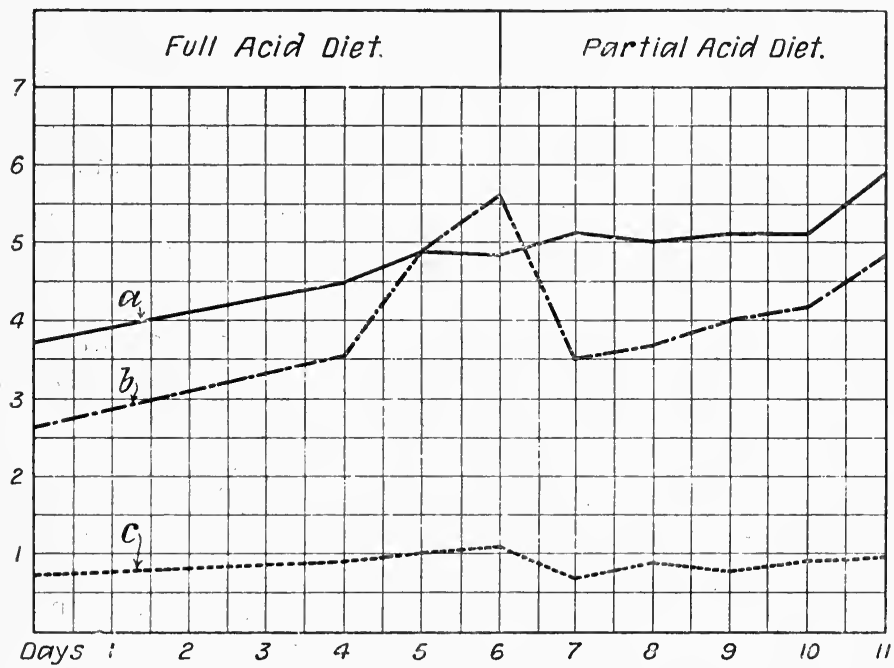

Fig. 64.-Chart Showing Increased Secretion and Alkalinity of Saliva to the Same Stimulus, i.e., Biscuit, during an Acid DiETARY.

$a$, Amount per minute; $b$, alkalinity per minute; $c$, alkalinity per c.c.

linity index. It is noticeable, too, that during the period of mixed diet the amount per c.c. is greater than during full acid diet. This introduces other factors in salivary secretions (and these are still under consideration) - the influence of the loss of water via the kidneys, the bowel, and the sweat glands; for of course the acid salts of fruits are, in large doses, diuretic, aperient, and slightly diaphoretic. It is interesting in 
this respect to note that between days 6 and 7 the alkaline salts of the saliva diminished, but the water increased during biscuit mastication.

There is obviously a most intimate relationship between the salivary, renal, intestinal, and sweat glands. If the excretory glands are functioning to an abnormal extent, the salivary glands are either reflexly or directly sensitive to a diminished water-supply; they secrete less, and thereby the mucous membrane of the tongue and mouth is rendered less moist. Thus, mechanically, an inducement to drink more fluids is brought about, the loss of water from the tissues is made good, and the salivary glands after a time resume their normal secretion. Here, then, we have another function of the salivary glands-to act as automatic indicators of the need for the intake of water.

I think, therefore, that the increased amount of saliva per minute during the second period is due to decreased loss of water via the above channels.

We have shown, then, how a feeble salivary stimulant may be converted into a strong one, and how an alkalinity index of 2.69 may be raised by dietetic means to one of 4.8 or 5.6 , and that of "resting" saliva from $\mathrm{I} \cdot 73$ to $3 \cdot 05$. As a means of permanently raising the alkaline reaction of the mouth, neutralizing and diluting the acids formed by fermentation, and so preventing the occurrence of caries, this procedure must be so self-evident as to need no comment; it is, moreover, eminently practicable.

These results are in accordance with the observations of Haidenhain,* that the augmenting effect of a strong electrical stimulus extended over a period after its cessation, and that a weak stimulus applied after a strong one induced a greater secretion than a weak one did originally.

*Pflüger's Archiv, xvii., r 878 . 


\section{Salivary Depressants.}

We now turn our attention to the other side of the question, and briefly consider those means by which the secretion and alkalinity of the saliva are depressed.

It has already been shown that such substances as bread and butter, tea, tannic acid, sodium carbonate, and meat, actually depress the salivary secretion below normal; and it is in accord with physiological principles to infer that the habitual consumption of such articles cannot but have a permanent effect upon the salivary glands in the direction of lessening their power for secretion. The general law that the frequent application of "overcomeable" stimuli is necessary for the development and full functions of all organs must apply to the salivary glands also, and the constant absence of such stimuli must tend in the other direction, to lack of development or atrophy. (This will be demonstrated subsequently by actual experimental evidence.)

There is a class of substances that have not yet been referred to, but which must act in this direction, that are not articles of diet, but of which enormous quantities in the aggregate are used by the public. I refer to tooth powders and pastes. Practically the universal base for these dentifrices is calcium carbonate, combined with other alkalies, such as sodium and magnesium carbonates and soap; a minute quantity of antiseptic and some aromatic flavouring agent are also usually included. They are used to " neutralize the acids of the mouth." In other words, they are used to do what it is presumed Nature cannot do; they are, as it were, a crutch for poor, feeble Nature to lean upon. It is as if one should in all cases of dyspepsia, irrespective of its cause, give constantly and continuously an artificial gastric juice, or, in the case of an enfeebled heart, insist on a patient maintaining the recumbent position until the heart became as strong as normal 
The action of such dentifrices on the salivary secretion is shown in Table VI. It is seen that a simple dentifrice without flavouring agents very considerably reduces the alkalinity and the amount of the saliva; and that, even when some of the powder remained in the mouth, the total alkalinity of the mouth five minutes afterwards was subnormal.

The addition of flavouring agents decreases the depression to a slight extent, but the resulting oral reaction is still markedly below normal. Sinilar tests have been made on a number of individuals, and always with the same result-a

\section{TABLE VI.}

Total Saliva Five Minutes after the Use of Dentifrices.

\begin{tabular}{|c|c|c|c|}
\hline Substances used as Dentrifice. & $\begin{array}{l}\text { C.C. per } \\
\text { Minute. }\end{array}$ & $\begin{array}{l}\text { Alkalinity } \\
\text { per C.C. }\end{array}$ & $\begin{array}{l}\text { Alkalinity } \\
\text { per } \\
\text { Minute. }\end{array}$ \\
\hline $\begin{array}{l}\text { Normal saliva (resting), to show in- } \\
\text { crease or decrease } \ldots\end{array}$ & $1 \cdot 65$ & 1.05 & $1 \cdot 73$ \\
\hline $\begin{array}{l}\text { Chalk, soap, and carbonate of soda, } \\
\text { with tooth-brush }\end{array}$ & $\mathrm{I} \cdot \mathrm{OO}$ & $0 \cdot 90$ & $0 \cdot 90$ \\
\hline The same used with finger, traces re- & $I \cdot I O$ & $1 \cdot 30$ & $1 \cdot 43$ \\
\hline $\begin{array}{c}\text { Chalk, carbonate of soda, cloves, and } \\
\text { gaultheria }\end{array}$ & $\mathrm{I} \cdot 3 \mathrm{O}$ & $\mathrm{I} \cdot \mathrm{OO}$ & $1 \cdot 30$ \\
\hline Acid potassium tartrate (powder) $\quad$. & $I \cdot 90$ & $\mathrm{I} \cdot 20$ & $2 \cdot 28$ \\
\hline
\end{tabular}

marked lowering of the alkalinity index. Comparing these results with that after the use of acid potassium tartrate as a tooth-powder, we see that the latter produced a considerable rise in amount per minute and alkalinity per c.c., and that the total alkaline reaction of the mouth five minutes afterwards is raised from an index of $\mathrm{I} \cdot 73$ to $2 \cdot 28$.

We cannot but conclude from this that the use of alkaline dentifrices for the prevention of caries is wrong, is physiologi.cally incorrect, unscientific, and empirical; and not only so, but is also actually conducive to the inception and progress of 
disease, by decreasing the circulation and alkalinity of fluids in the mouth.

The use of alkalies seems to be based upon a wrong conception. It is as though it were thought that lactic acid developed and accumulated in the mouth, remaining there for some hours or until next morning, when an overwhelmingly strong alkali is introduced to neutralize it; whereas, of course, as each molecule of lactic acid is formed, it searches for something wherewith to combine. Alkaline salts of the saliva will obviously, most readily satisfy it, but should these not be available, then the calcium phosphates and carbonates of the enamel surface are attacked.

It cannot be too clearly recognized that, by the use of alkalies, only those molecules of acid formed immediately previously can be neutralized, and also that the natural defensive forces of the mouth are thereby lowered for some considerable time afterwards.

\section{Diminished Taste Perception.}

It would seem not to be wrong to infer that the habitual consumption of substances which fail to stimulate the endings of the lingual and glosso-pharyngeal nerves in the tongue, especially during the years of development, would lead to their becoming less excitable or dulled in their appreciation of the different taste stimuli. There are substances, too, such as alcohol and tobacco in excess, which, as is well known, interfere with the sense of taste. Nicotine applied experimentally to the glosso-pharyngeal nerve will prevent the reflex secretion of saliva upon stimulation.

Assuming, then, for the present that this does take place, the following experiments were, done to determine what the effect would be on salivary secretion. The condition of dulled taste-appreciation was artificially produced by painting various parts of the tongue with cocaine. Naturally this 
at first produced a profuse flow, owing to its bitter taste; but it rapidly passed off, and after ten minutes the saliva was collected during mastication and observed. Table VII. shows the results. When the anterior two-thirds of the tongue are cocainized, there is an obvious diminution of both total amount and alkalinity ( $c f$. Table II.).

Cocainizing the posterior third of the tongue produces even a greater diminution in both amount and alkalinity of the total saliva during biscuit mastication, the depression being chiefly in the amounts per minute from each gland. During apple mastication the decrease from the normal ( $c f$. Table I.) is very considerable from all the glands in both amount and alkalinity, the total alkalinity per minute being reduced by 70 per cent.

The results of partially cocainizing the whole tongue are very much the same as in the latter case, the decrease in amounts per minute being more affected than the alkalinity per c.c. ; but the alkalinity index is even more reduced than by cocainizing the posterior third alone.

That similar states may be present abnormally in the mouth seems to be extremely probable. I have estimated accurately by titration the perception of such tastes as sweet, bitter, and acid, in about a dozen individuals; and although this number is insufficient from which to form definite conclusions, it is sufficient to show that the sensitiveness of the nerve endings of the tongue does vary perceptibly in different individuals, and, moreover, that a dulled taste-sensibility and marked susceptibility to caries seem to be associated.

Salivary depression may be induced psychically. Pavlov* has observed that strong excitement in a dog may be sufficient to entirely suppress the flow of saliva. The same thing is well known to occur in the human subject. The effect of strong mental concentration is always to produce a condition of " dry 
THE SALIVA

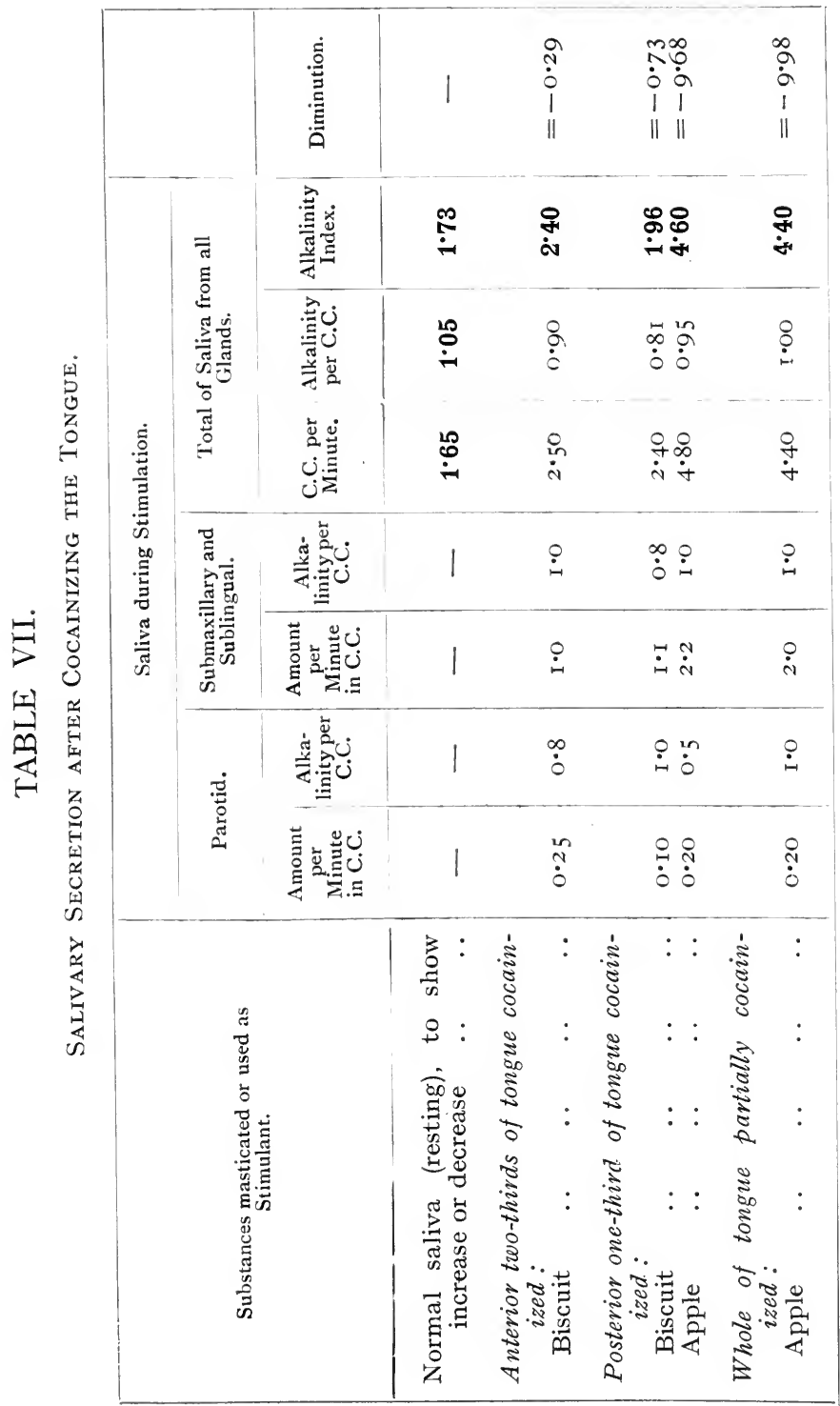


mouth ;" the urinary secretion is stimulated, but the salivary secretion is depressed.

On the other hand, strong muscular exertion produces at first a much increased flow of saliva, followed some time afterward by a diminution and a sense of dryness, due, most probably, to loss of water from the body via the sweat glands.

Here, then, we find two concomitant conditions of higher civilization-i.e., increased mental excitement and subnormal muscular exercise-both tending in the direction of decreased salivary secretion.

It has been noticed* in Madeira that boys who spend a considerable portion of their time in diving for copper coins thrown into the sea for them by passengers on ships usually have extensive caries of the teeth. This is interesting in that it is, I think, undoubtedly associated with the marked astringent effect which copper has in the mouth. Further, it is of course a powerfully antiseptic metal; but obviously this property is unavailing to prevent caries occurring.

It has frequently been noted that climatic changes-a rapid change, for instance, from the Temperate to the Torrid Zoneis followed by a rapid increase in the progress of dental caries. This may be due, to a certain extent, to a change in diet, but is more likely to be due, I think, to the disturbance of the established balance between the kidneys, sweat, and salivary glands, acting in nearly all cases in an excitation of the two former and a depression of the latter. This is the more probable, since Englishmen at least, as is well known, insist as far as possible upon an " English " dietary wherever they may be, instead of adapting themselves to that dietary which the process of evolution has demonstrated to be the most fitted for the particular locality.

The Effect of "Common Colds." - It is well known that in a

* British Journal of Dental Science, 1906, p. 365. 
common cold the perception of taste is very much diminished. "Things taste as if they had no taste." This, of course, is accompanied by a much decreased salivary flow, which deprives the teeth of protection at a time when it is much needed-firstly, because of the "sloppy" character of the diet at these times; and, secondly, on account of the increased activity of the bacterial flora in the nose and mouth.

"The frequency of the general incidence of " common " and " influenza" colds at the present time undoubtedly lias a causal relationship to the incidence of caries.

It is significant to note that when one is suffering from these complaints there is generally a natural craving for fruit and acid drinks. This desire should always be gratified as far as it possibly can be.

\section{Experimental Evidence from Lower Animals.}

A series of experiments has been carried out in rabbits to determine the possible effect on the salivary glands of decreased stimulation of the gustatory nerves. Rabbits were chosen for the purpose because in them the salivary glands must be a great necessity, as they do not drink.

I. The effect of softened and partially neutralized food.

(a) Two rabbits of equal age (two months) were selected A was fed on cabbages, turnips, carrots, and grass, previously boiled in weak sodium carbonate solution; B was fed on the same food, but unboiled and in the natural condition. At the commencement of the experiment A weighed 6I7 grammes, and B weighed 602 grammes. The experiment continued for a little over four months (nineteen weeks). At the end of this time A weighed I,275 grammes and B weighed I,077 grammes. The animals were killed, and the submaxillary glands removed and weighed. The mean weight of the submaxillary glands of A was 0.4250 gramme; the mean weight of the submaxillary glands of B was 0.4325 gramme. Thus it is seen that, although 
A was 200 grammes heavier than B, it had the smaller salivary glands. Expressing these as weights per kilogramme of bodyweight, we have $\mathrm{A}=0.3336$ gramme, $\mathrm{B}=0.4004$ gramme; a slight difference, it is true, but still quite appreciable, especially considering the comparative shortness of the time (nineteen weeks). The submaxillary glands only were examined, since in the rabbit the parotid gland is said to secrete continuously, irrespective of stimulation.

(b) An exactly similar experiment was commenced with two other rabbits, very much younger (two to three weeks), in order to avoid the initial " education " of the gustatory nerves, and the subsequent possible "memory reflex secretion." C was fed on boiled neutralized food, and weighed at the commencement 285 grammes. D was fed on similar food, untreated, and weighed at the commencement 259 grammes. Unfortunately, at the end of a fortnight $\mathrm{D}$ was killed by another rabbit; $\mathrm{C}$ afterwards developed most marked rickets in all its long bones, and the front-feet became completely retroverted. At the end of three months (twelve weeks) it was killed. At the time of death $\mathrm{C}$ weighed 485 grammes, and the mean weight of the submaxillary glands was 0.2125 gramme. D, when it was accidentally killed, weighed 270 grammes, and the mean weight of the submaxillary glands was 0.2362 gramme.

If these may be compared, then it is seen that, although $C$ was four times the age and nearly twice the weight of $\mathrm{D}$, yet its salivary glands were very appreciably less in weight.

2. The artificial elimination of the gustatory stimuli.

Rabbits were anæsthetized (with chloroform), and various portions of the mucous membrane and subjacent tissue of one side of the tongue were removed with the electric cautery. After the lapse of a few months the weight of the glands on either side was compared. The left side of the tongue was 
always selected, since Bidder and Haidenhain* found that, in the dog at least, the left submaxillary glands are as a rule larger than the right.

(a) A full-grown wild rabbit (weight I,I40 grammes) was anæsthetized, and the whole of the mucous membrane and submucous tissue of the left side of the tongue was removed with the electric cautery. The rabbit made a good recovery, and was fed on ordinary fresh food for a period of two months. It was then killed, and the glands removed and weighed, with results as shown in the following table:

\begin{tabular}{|c|c|c|}
\hline Weight of Right Gland. & Weight of Left Gland. & Weight of Rabbit. \\
\cline { 2 - 4 } or 0.558 gramme & 0.4300 gramme & I, I60 grammes. \\
0.48 I, & $0.3793 \quad$, & $\begin{array}{c}\text { expressed as per kilo } \\
\text { of body-weight. }\end{array}$ \\
\hline
\end{tabular}

Thus it is seen that at the end of two months the gland receiving its stimulus from the side of the lesion was appreciably less in weight than that on the normal side, the diminution being one of o.IoI7 gramme per kilo of body-weight.

(b) Another full-grown wild rabbit, weighing I,299 grammes, had the mucous membrane removed from the anterior twothirds of the left side of the tongue in a similar manner. It was fed on precisely similar food to the other rabbit, and was kept for the same length of time (two months). The following were then the weights of the glands:

\begin{tabular}{|c|c|c|}
\hline Weight of Right Gland. & Weight of Left Gland. & Weight of Rabbit. \\
\cline { 1 - 2 } $\begin{array}{c}0.52 \text { Io gramme } \\
\text { or } 0.4 \text { I I I , }\end{array}$ & $\begin{array}{c}0.4762 \text { gramme } \\
0.3670 \quad,\end{array}$ & $\begin{array}{c}\text { 1,2 70 grammes. } \\
\text { expressed as per kilo } \\
\text { of body-weight. }\end{array}$ \\
\hline
\end{tabular}

* Quoted by J. N. Langley in Schäfer's "Textbook of Physiology," p. 487 . 
Here again is a distinct difference between the two glands, the diminution in this case amounting to $0 \cdot 044 \mathrm{I}$ gramme per kilo of body-weight, or a little less than half the diminution observed when the whole of the mucous membrane was removed. If any inference could be drawn from this, it would be that the stimulation arising from the excitation of the glosso-pharyngeal nerve is of somewhat greater importance than that from the lingual nerve.

(c) A full-grown tame rabbit was also the subject of an exactly similar experiment to the last. The operation, however, seemed too severe for it. A curious form of toxæmia was set up, and the animal died with convulsions in one week. Although, therefore, the experiment may be said to have failed, it is interesting to note that in this case also the gland on the side of the operation was 0.059 gramme less than the other.

In all the above experiments the animals were killed in the fasting state, when the glands should be at their largest.* In all the tongue experiments the operation caused immediately great salivation and very marked swelling of the submaxillary gland of that side. The increase in the size of the gland was both palpable and obvious. On the third day, however, the salivation and swelling disappeared almost suddenly.

In these experiments, although the differences in the weights of the gland are not great, yet they are fairly constant; and, moreover, one has to remember (I) the comparatively short duration of the loss of excitation; (2) the effect of the psychical stimulation, and this would probably be increased by the fact of one side of the tongue being normal; (3) the fact of the animals being full-grown.

An endeavour has been made to eliminate the age and the psychical factors by selecting quite young animals, but up to the present the experiments have failed.

* J. N. Langley, in Schäfer's “'Textbook of Physiology," p. 487. 
Efforts were made to estimate the amount and character of salivary secretion in the above series of rabbits during life, by collecting the saliva by aspiration from the mouth after the injection of pilocarpine (I milligramme per kilo of bodyweight). Although this method cannot be relied upon for anything like absolute accuracy, yet it may be noted that on three out of four occasions the rabbit fed on boiled neutralized food yielded from one-half to one-third less saliva per minute than the normal rabbit, and also the percentage of alkaline salts and total alkalinity per minute were correspondingly diminished. With regard to the wild rabbits, the element of fright was so predominant as to make the results too variable to be of any value.

It may be concluded, therefore, that in animals a diminution in the excitation of the gustatory nerves leads to a loss of weight in the salivary glands, and presumably also to a corresponding loss of function.

\section{Note on the Quantitative Difference between Parotid and Submaxillary Secretion.}

The previous experiments relating to salivary secretion show that the reflex secretion from the parotid gland is always considerably less than that from the submaxillary and sublingual glands. This is certainly not what might be expected, if we have in mind the size and structure of the parotid gland. I have suggested that this comparatively small secretion of the parotid might be causally related to the increased amount of caries found in upper teeth (ratio $3: 2$ ). I have therefore endeavoured to find a substance which might have some selective effect on this gland, but so far without very much practical success. The gland does not seem to be under quite the same nervous control, or under such control to the same extent, as the submaxillary. The substances which give the best results are strong acids-acetic, 
citric, malic; and this coincides with what was found by Carlson and Crittenden, and by Sellheim in the dog. But in order to produce this increase, the acids have to be used in what would be too strong a solution for ordinary use.

Reflex stimulation of the cranial nerves-lingual and glosso-pharyngeal-fails, then, ordinarily to invoke any very considerable flow of parotid saliva.

It might be thought, therefore, that the activity of this gland was perhaps induced normally by stimuli via the sympathetic nerves. The following experiment by Langley,* however, disproves this, for, as is shown, sympathetic stimulation practically inhibits secretion.

Dog. Pilocarpine Nitrate injected. Rise of Saliva in Tubes Connected With the Ducts of the Submaxillary and Parotid Glands taken every Thirty Seconds, in Millimetres.

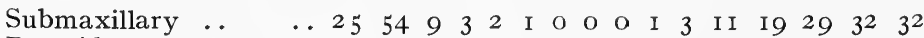
$\begin{array}{llllllllllllllllllll}\text { Parotid ... } & \ldots & \ldots & 5 & 9 & 2 & 0 & 0 & 0 & 0 & 0 & 0 & 0 & 0 & 0 & 0 & I & 2 & 2\end{array}$ Sympathetic stimulation.

(This "sympathetic" effect is, I believe, of considerable pathological importance, and has formed the basis of experiments on blood-pressure and intestinal stasis, the results of which will be found in a later chapter.)

Neither does a substance like pilocarpine circulating in the blood-stream raise the parotid secretion to ariything like the same level as the submaxillary, as may be seen above.

In the experiment quoted it will be seen that the proportion of submaxillary to parotid saliva is slightly more than Io: I in the dog, my own results for the human glands being $5 \cdot 8:$ I. Sellheim $\uparrow$ found that by producing saliva reflexly from the mouth in the dog the ratio was $2 \cdot 2: \mathrm{I}$.

That it is the parotid secretion which is proportionately low, especially in man, is confirmed by the fact that Carlson

* Journal of Physiology, vol. x., p. 826.

$\uparrow$ American Journal of Physiology, April I, I9Io. 
and Crittenden found that the average secretion of the parotid to weak, medium, and strong acids was 0.34 c.c. per minute, and to weak acids alone my own figures give an average of 0.40 c.c. per minute.

Sellheim's figures for the dog give an average of $3 \cdot$. c c.c. of submaxillary and sublingual saliva per minute from one side, and my own estimations for the same glands in the human being give an average of $2 \cdot 3$ c.c. per minute, also from one side only.

Thus it will be seen that experimental evidence is fairly uniform in proving that the parotid saliva is always very much less than the submaxillary, and that the gland is resistant of ordinary stimulation, but that it may be considerably depressed.

The precise mode of normal stimulation is therefore still to find. It cannot be doubted that so large a gland must have greater function than that of pouring out the relatively small secretion in response to usual reflex stimulation in the mouth. May it be that its function is to secrete a very small quantity continuously instead of large quantities periodically, as it is said to do in the rabbit? Or may it be that its full function is only brought into action when the mouth is required to be cleansed of some particularly obnoxious material? Pavlov found that the only things which produced a copious parotid secretion in the dog were very dry articles-dry sand and dry powdered flesh. Again, dentists frequently have ocular evidence of rapid parotid secretion during excision of sensitive dentine. It would seem, therefore, not unlikely that the proper stimulus for the parotid is abnormal stimulation of fifth nerve.

\section{Neutralizing Power of Saliva.}

In discussing the reaction of saliva, it is always necessary to state the indicator used, since saliva gives quite different reactions with various indicators. For instance, the general 
rule is that all salivas are acid to phenolphthaleïn, owing to the contained carbon dioxide and bicarbonates, but that all salivas are alkaline to methyl orange, owing to this indicator not being affected by carbon dioxide; whilst the same salivas may be either acid or alkaline, or both (amphoteric), to litmus, owing to the presence of acid phosphates.

Can saliva neutralize lactic acid and prevent the decalcification of enamel? It has been suggested that this does not, or cannot, occur. The following facts should answer the question satisfactorily. Head* found that, whilst a weak watery solution of an acid phosphate produced distinct decalcification of enamel, a similar solution in saliva produced no effect at all.

Experiments have been carried out here also with similar results. Solutions of acids in saliva were made, and teeth with the enamel only exposed placed in them for varying periods, with the following results:

\begin{tabular}{|c|c|c|c|c|}
\hline $\begin{array}{l}\text { Acid added to } \\
\text { Saliva. }\end{array}$ & Indicator. & Reaction. & Time. & $\begin{array}{l}\text { Effect on } \\
\text { Enamel. }\end{array}$ \\
\hline Lactic & Methyl orange & Neutral & 9 days & None. \\
\hline Lactic & Methyl orange & $\begin{array}{l}\text { Very slightly } \\
\text { acid }\end{array}$ & 9 days & None. \\
\hline Lactic & Methyl orange & Acid & 3 days & $\begin{array}{l}\text { Soft opa- } \\
\text { city. }\end{array}$ \\
\hline Lactic & Litmus & Neutral & ro days & None. \\
\hline Sulphuric & Litmus & Neutral & Io days & None. \\
\hline Sulphuric & Methyl orange & Neutral & Io days & None. \\
\hline Sulphuric & Methyl orange & Faintly acid & Io days & None \\
\hline $\begin{array}{l}\text { Lactic (in } \\
\text { water) }\end{array}$ & Congo red & Acid (blue) & 45 days & Decalcified. \\
\hline Lactic & Congo red & $\begin{array}{c}\text { Partially al- } \\
\text { kaline } \\
\text { (red) }\end{array}$ & 45 days & None. \\
\hline Lactic & Congo red & Violet & 45 days & None. \\
\hline
\end{tabular}

It is quite obvious, therefore, that, in terms of the ordinary indicators used, saliva does neutralize lactic and other acids, 
and, further, that such products as may be formed-acid phosphates-are not capable under such conditions of producing any decalcification of the enamel surface.

\section{Ptyalin.}

The fact that ptyalin may act as a protective agent for the teeth seems to have been overlooked, though on consideration it would appear to be quite clear that this is so. The function of ptyalin is to convert starch into sugar; and although Cannon* has shown that the salivary digestion of starch may go on in the stomach for some time after its being swallowed, yet there is no doubt that the amount of starch so converted is, in proportion to the total amount eaten, extremely small.

The saliva of many animals contains no ptyalin, notably in the carnivora, including the dog; yet the latter digests starches very completely. The saliva of the horse, too, I have ascertained, possesses extremely feeble diastatic properties, yet this animal exists very largely on starch.

If, then, the salivary digestion of starch in the stomach is of but little importance, why is ptyalin present in saliva? There can be but one reason-in order to digest starch débris in the mouth. In other words, to convert a solid, insoluble and adhesive substance like starch into a very soluble and easily removable substance, such as is maltose, so as to prevent stagnation and promote circulation.

Now, since maltose is probably more easily fermented by the mouth organisms alone than is starch, it will obviously be of advantage either to have no ptyalin at all present or to have a considerable amount, so that either the organisms should not be aided in their work or that their pabulum should be rapidly swept away. Moreover, the conversion from starch to maltose is not a simple process; erythro-

* American Journal of Physiology, 1904. 
dextrin and achroo-dextrin are intermediate products. Now, the sticky, adhesive nature of dextrin is well known; and if the ptyalin be small in amount the conversion process will be a correspondingly slow one, the dextrin formed will remain longer, and this will tend to bind the substance closer and more firmly to the teeth. Again, free organic acids destroy the diastatic action of ptyalin; if, then, when the starch is slowly and partially converted into maltose and dextrin, the organisms incorporated in the mass form free lactic acid, no further action of the ptyalin takes place, and the starch is literally gummed to the teeth, to be slowly but surely transformed into lactic and other acids.

On the other hand, if the action of the ptyalin is much more rapid than that of the organisms, there will be a fair chance of all the starch being converted into maltose, and so carried away, provided there is adequate salivary secretion. Now, although in the case of ferments the actual amount of substance eventually converted does not depend upon the amount of ferment present, yet the rapidity of that action, especially in the initial stages, does depend upon the amount and its concentration. It is therefore of advantage to have present in the mouth as large an amount of ptyalin per minute as possible.

The ratios of these amounts, in salivas produced by a series of dietetic stimulants, have been determined. The method used was that of Mett's tubes. Fine glass tubing-not quite capillary-is filled with starch coloured blue with iodine. The tubing is then divided into short lengths of about I cm. and placed in the sample of saliva to be tested. (The tubes are kept horizontal, and the capsules covered to prevent evaporation.) The saliva is then incubated for one hour at $36^{\circ} \mathrm{C}$., and the amount of starch converted-i.e., amount of disappearance of blue coloration from each end of the tubeis read off under the microscope by means of an eyepiece 
micrometer and very low power objective. The method gives very constant results. The amount of ptyalin, however, is not directly proportional to the measurement so obtained. Schütz and Borisov have formulated a law, endorsed by Pavlov,* that a ferment in a fluid varies with the square of the length of the column of digested starch measured in millimetres. This law has been in the present instance applied to saliva, and the amount of ptyalin present under the several conditions is indicated by such numerical ratios in Table VIII. Reference to this table shows that the concentration of the ptyalin in the parotid saliva is in all cases (but one) very much in excess of that in submaxillary and sublingual glands.

The total ferment units for each gland are obtained by multiplying the rate per minute by the square of the length of digested starch. If this be done, it will be found that in many cases the deficiency in concentration in the submaxillary glands is more than made up for by the increased rate of flow, so that it is doubtful whether we should continue to regard the parotid gland as the one chiefly responsible for the ptyalin content of the saliva.

The substances are arranged in the order of their ptyalin indices (total ferment units from all glands per minute), and it will be noted that the order is practically the same as that in which they were arranged for the inorganic salts. It is observed that acids increase the ptyalin index more than ten times that of the " normal resting" index.

It is remarkable that the index for " biscuit saliva " is only increased by $3 \cdot 6$, yet it is for this class of carbohydrate that the beneficial solvent action of pytalin is most required. Does this not show that the environmental forces of civilization have not yet produced a physiological adaptation to highly artificial or civilized articles of diet, as opposed to "natural" foods like fruit? Undoubtedly this is so in a

* American Journal of Physiology, p. 30. 


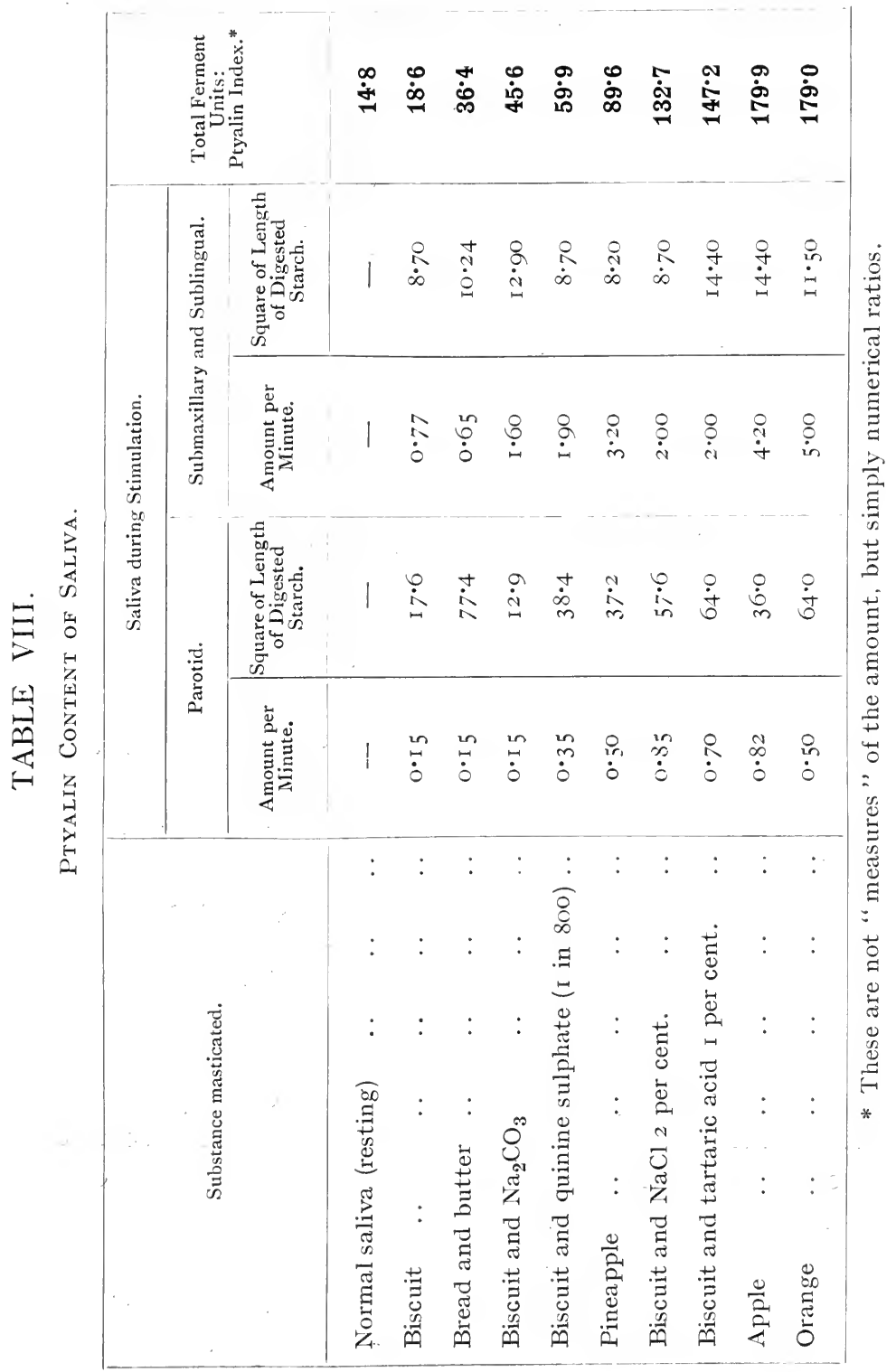


large measure, yet not entirely so, for it is seen that the greatest concentration of ptyalin from the parotid gland is present in the saliva produced by bread-and-butter excitation; and since the rate per minute is the same for biscuit, bread and butter, and alkaline biscuit, there evidently is a special stimulus from bread and butter which induces a greater secretion of ptyalin. To this extent, then, adaptation has occurred, but when the total ferment units per minute are compared with those for acid biscuit and acid fruits, like apple and orange, it is evident how feeble the adaptation really is.

The mere addition of acid to the biscuit increases the ptyalin index eight times. In fact, the ptyalin secretion seems to be much more responsive to the stimulus of acids than any other constituent of saliva.

The diminution in the concentration of parotid ptyalin from alkaline biscuit is very marked. The addition of the soda has reduced it by 4.7 ; an increase, however, in the amount of saliva per c.c. from the submaxillary glands makes up for this in the total.

We cannot but conclude that in this demonstrably diminished ptyalin for ordinary starchy foods we have one of the potent causes of the present prevalence of dental caries. Enormous quantities of carbohydrates in such forms are consumed, and, as we shall show, they are also the forms which both adhere most to the teeth and from which most acid is capable of being formed. These are therefore the articles of diet which require the greatest solvent action of ptyalin, in order that they should be washed away before acid is developed - yet they are the ones which receive least.

Fortunately these experiments also show a way out of the difficulty. It is, obviously, to increase the ptyalin index by using dilute organic acids incorporated with the carbohydrates artificially; or by the liberal use of acid fruits and fresh veget- 
ables, salads, etc., to raise the rate of salivary secretion, and also the excitability of the salivary glands to weak excitants like bread and biscuit (see.Table V.). This will have the double effect of dissolving the starch and of washing away the resulting maltose.

Regarded from this point of view-that of cleansing the mouth and protecting it from disease-the presence of ptyalin in saliva becomes rational and comprehensible. It indeed fulfils a function which nothing else could possibly do: it renders soluble an insoluble and adhesive substance like starch, and thus prepares it for being naturally washed away. No substance prepared by the wit of man could possibly produce such an effect. No dentifrice or mouth-wash can directly remove the starch débris in such a manner; it can only cleanse mechanically, and the majority of such artificial detergents have, as it has been shown, a depressant effect upon the salivary secretion.

\section{Extirpation of the Salivary Glands.}

The salivary glands have been excised in the dog by Fehr,* Minkowski, † and Schäfer and Moore. $\ddagger$ In all cases the animal survived, and the operation produced no visible effect, except - that in one case it drank more water than before. The undigested starch in the fæces was not increased in amount, and no sugar appeared in the urine.

Having regard to the fact that ptyalin in dog's saliva seems at most to be only occasionally present, it seemed to the author that this experiment should be repeated in an animal which would not drink more water after excision of the glands, and in which ptyalin was normally present in the saliva. A rabbit was therefore chosen as the subject of the experiment. It

* Archiv für Exper. Path. u. Pharmakol, Leipzig, I893, Bd. xxxi., S. 14 I. $\dagger$ Ibid.

$\ddagger$ Journal of Physiology, Cambridge and London, . 1896, vol. xix., p. xiii. 
was found that the undigested starch in the fæces was not constant in a normal rabbit under ordinary conditions, but varied slightly on different days, this depending possibly on some slight difference in the diet; but it was also found that the undigested starch in the fæces of rabbits living under similar conditions varies similarly; hence, instead of comparing the undigested starch in the fæces of one rabbit before and after the operation, comparison was made with a control rabbit of the same age and weight (approximately), and fed on precisely the same food.

Two white tame rabbits, $A$ and $B$, were selected, their weights being respectively $\mathrm{I} \cdot \mathrm{I} I 9$ kilogrammes and $\mathrm{I} \cdot 245$ kilogrammes. B was anæsthetized with chloroform, and the parotid and submaxillary glands on both sides were excised by two curved incisions, about half an inch below the inferior margin of the lower jaw (the sublingual glands were not removed, since these are quite small and very difficult of access in the rabbit owing to the narrowness and depth of the lower jaw). The wounds were closed and healed aseptically.

For two days after the operation the interior of the mouth (with which the wounds did not communicate) was much swollen and inflamed, due, doubtless, to a surface infection and to the irritant action of the food, since the rabbit's appetite and ability to eat did not seem to be at all diminished. This, however, subsided, and the rabbit made an uninterrupted recovery, there being no difference in any respect between it and the control. During the whole time the rabbits were fed on as " natural " food as possible-i.e., grass, clover, dandelions, etc.

Seven weeks after the operation the weights of the rabbits were: $A=I \cdot 559$ kilogrammes, and $B=I \cdot 698$ kilogrammes. The fæces of both rabbits were collected at intervals, and the undigested starch estimated by the following method: 5 grammes of dried and pulverized fæces were placed in a flask 
containing 200 c.c. of water and 7 c.c. of hydrochloric acid; the flask was fitted with a condensation tube, and was kept on a sand bath at $100^{\circ} \mathrm{C}$. for two days. In this manner any starch present became hydrolyzed into glucose. The contents of the flask were then cooled, neutralized, and filtered, and the filtrate made up to 500 c.c. The glucose present in this was then estimated in the usual manner with Fehling's solution, the results being expressed in terms of starch.

Four estimations of each were made on each occasion. It was found that there was practically no difference between the amount of undigested starch present in the fæces of $\mathrm{A}$ and B.* The following were the results of three examinations:

\begin{tabular}{|c|c|c|c|c|c|}
\hline $\begin{array}{c}\text { Amount of Glucose } \\
\text { present in } 5 \text { Grammes } \\
\text { of Fæces. }\end{array}$ & & & & \multicolumn{2}{|c|}{$\begin{array}{l}\text { Expressed as Per- } \\
\text { centage of Undigested } \\
\text { Starch in Freces. }\end{array}$} \\
\hline I. $\mathrm{A}, \mathbf{I} \cdot 38$ & $\cdots$ & $\cdots$ & $\cdots$ & . & $24 \cdot 80$ \\
\hline $\mathrm{B}, \mathrm{I} \cdot 40$ & . & . & . & . & $25 \cdot 20$ \\
\hline 2. A, I.22 & $\cdots$ & $\cdots$ & $\cdots$ & - & $2 I \cdot 90$ \\
\hline $\mathrm{B}, \mathrm{I} \cdot 22$ & . & . & . & $\cdots$ & $2 \mathrm{I} \cdot 90$ \\
\hline 3. $\mathrm{A}, \mathrm{I} \cdot \mathrm{I} 4$ & $\cdots$ & - & . & $\cdots$ & $20 \cdot 52$ \\
\hline $\mathrm{B}, \mathrm{I}^{\bullet} \mathrm{I} 3$ & . & . & . & . & $20 \cdot 34$ \\
\hline
\end{tabular}

Mean percentages: A, $22^{\circ} 4^{\circ} 3 ; \mathrm{B}, 2^{\circ} 4^{\circ}$. Difference: $0^{\circ} 07$ per cent.

It will be observed that on one occasion the percentage of undigested starch in the freces of $B$ was slightly in excess of $A$, on one occasion $A$ was a trifle in excess of $B$, and on the other occasion both were exactly equal. The mean percentages show a difference of 0.07 per cent. in favour of $B$. Having regard, however, to the fact that this was not constant, and that the difference is so small as to come probably within the range of experimental error, it may be concluded that the excision of the salivary glands did not affect the digestion of starch in the alimentary tract.

The inference from the results of such experiments upon animals is to still further strengthen the conclusion that

* This experiment has been repeated with the addition that the orbital salivary glands were also excised. The result was similar: the digestion of starch was not decreased in the slightest. 
one of the chief functions of ptyalin is to act as a protective agent in the mouth, since it has quite a negligible value as a digester of starch for absorption in the intestine.

\section{Digestion of Starch by Ptyalin.*}

The secretion of acid gastric juice never commences until five minutes after food has been eaten. The cause of this latent period is unknown, but Pavlov has suggested that it serves to give time for the ptyalin to freely digest starch in the stomach. As previously stated, Cannon has observed that the stomach contents do not become acid for forty minutes after the ingestion of food: this period also, therefore, is available for the action of ptyalin. I have accordingly performed a series of experiments to ascertain the amount of starch which may be digested in these times. The results are given by the following figures:

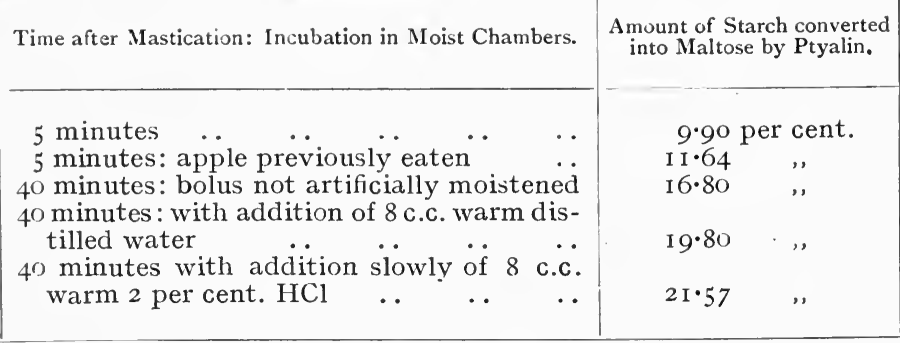

Thus it is seen that an appreciable amount of starch may be digested in these periods, and also that the addition of hydrochloric acid representing gastric juice has a beneficial effect, as, indeed, has been pointed out by Chittenden. Nevertheless, it is still quite obvious that, from the point of view of alimentary digestion of starch, ptyalin plays but a subsidiary part, even under the most favourable circumstances.

* Qualifying note added to the second edition. 


\section{Sulphocyanates.}

A considerable divergence of opinion exists as to the relationship of a marked amount of sulphocyanate in the saliva to a relative caries immunity.

The amount of the salt in human saliva is estimated variously as follows:

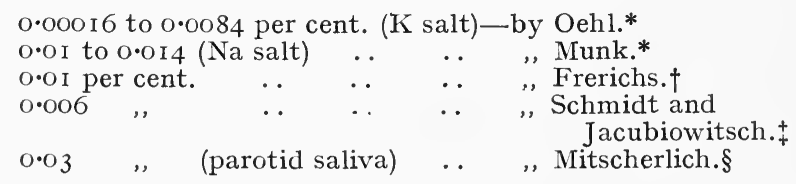

Ellenberger and Hoffmeister state that it is absent from the saliva of the horse, ox, sheep, goat, and pig, and that its presence is not constant in the dog. It is therefore clear that it is only present in minute quantities in man, and that it is absent from many animals that are immune to caries.

Sulphocyanate is claimed by some investigators to exert a continuous disinfectant action on mouth organisms, and so lessen the tendency to caries.

Michel || (of Würzburg) has tested the saliva of 840 patients, and finds that the amount of sulphocyanate and the incidence of caries are in inverse proportion. Low and Beach examined 300 patients, and came to a similar conclusion. Muntz and Hecht** are of a similar opinion.

Geyger (of Leipzig) recommends the internal administration of the salt in a dose of 0.008 gramme four times a day, and claims that thereby the course of rapid caries is checked and the teeth rendered less sensitive. In opposition to these views are the investigations of Florian, Chouppe, Hugen-

* Quoted by B. Moore, Schäfer's "Textbook of Physiology."

† Wagner's " Handwörterbuch d. Physiologie," iii. 758.

† Ann. Chem. Pharm., xxix. I 56.

$\S$ Halliburton's "Handbook of Chemical Physiology and Pathology," p. 622.

II Dental Cosmos, 1909, p. I 278 et seq.

- Ibid. 
schmidt, Miller, and Kirk. These authorities all agree in finding that any beneficial action arising from the presence of potassium sulphocyanate in the saliva is either entirely absent or is only extremely slight. Miller and Kirk are of the opinion that the presence of the salt in the saliva of persons relatively immune to caries is to be regarded as a coinci dence, and not the cause of the immunity.

Oppenheim* found that 0.5 per cent., I per cent., and 2 per cent. solutions of sulphocyanate had no sterilizing effect upon some fermentation bacteria. These solutions are, obviously largely in excess of the strength of the salt in the saliva.

In order to test the possible effect of potassium sulphocyanate in checking the fermentation of carbohydrate by mouth organisms, the following test has been made:

Six tubes, each containing Io c.c. of 2 per cent. dextrose (with litmus), were taken, and to five the following amounts of sulphocyanate were added: 0.00I, 0.005, 0.007, 0.0I, o.I gramme. To the sixth no sulphocyanate was added. The tubes were sterilized, and each inoculated with 0.2 c.c. of mixed saliva, and then incubated. In twenty-four hours all except the $0 \cdot I$ gramme tube showed an acid reaction (all were faintly alkaline at the start). In forty-eight hours the acid formed was estimated, with the following results:

\begin{tabular}{|c|cc|}
\hline $\begin{array}{c}\text { Percentage of Sulpho- } \\
\text { cyanate in each tube. }\end{array}$ & Turbidity. & $\begin{array}{c}\text { Acidity equal to C.C. } \\
\frac{\mathrm{N}}{50} \mathrm{H}_{2} \mathrm{SO}_{4} .\end{array}$ \\
\hline & & \\
Control & +++ & $1 \cdot 00$ \\
$\mathrm{O} \cdot \mathrm{OI}$ & -++ & $0 \cdot 5 \mathrm{O}$ \\
$\mathrm{O} \cdot \mathrm{O} 5$ & ++ & $0 \cdot 40$ \\
$\mathrm{O} \cdot \mathrm{O} 7$ & + & $0 \cdot 3 \mathrm{O}$ \\
$\mathrm{O} \cdot \mathrm{IO}$ & + & $0 \cdot \mathrm{I} 5$ \\
$\mathrm{I} \cdot \mathrm{OO}$ & Very slight & Neutral \\
\hline
\end{tabular}

At the end of a week the I per cent. tube had become markedly turbid and acid, and the acidity, upon estimation, 
gave a value of $0 \cdot 6$. Thus it would seem clear that sulphocyanate of potassium does exert a slight antiseptic action, although it is not sufficient, even in a I per cent. solution, to act as a disinfectant. It does also retard the process of fermentation, and lessens the amount of acid production in the strength in which it may be found in the saliva-i.e., o.or per cent.--decreasing the acid production in forty-eight hours by one-half. Obviously, then, there is a possibility that the constant presence of sulphocyanate in the saliva may have a beneficial effect, if only it is present in sufficient quantity.

In order to determine whether the concentration of sulphocyanate varied with the character of the stimulus similarly to other constituents, estimations have been made with a similar series of excitants. The percentage compositions of the samples of saliva obtained were estimated colorimetrically. A series of glass tubes are filled with varying percentages of potassium sulphocyanate from 0.0075 per cent. to 0.03 per cent. One tube also is used as a control, containing only distilled water. A dilute solution of ferric chloride acidulated with hydrochloric acid is added in equal quantities to each, the result being varying shades of colour, from amber $(\mathrm{o} \cdot \mathrm{CO} 5$ per cent.) to a light port-wine colour $(0.03$ per cent.), the control tube having a very light lemon colour. These shades are very constant, and are not altered by an excess of the ferric chloride. It is extremely important to note, however, that a really red port-wine colour is not given under 0.04 per cent.

Ferric-chloride solution and hydrochloric acid are then added to the saliva to be tested, and after centrifuging this, in order to clear it, the colour is matched against the series of known percentages. In order to eliminate the psychic factor, the tubes should be (and were always in these estimations) submitted to an independent observer. The test series of tubes require making up afresh each day, since upon standing for some hours the colours markedly fade. 
The presence of the sulphocyanate is confirmed by the addition of hydrochloric acid causing no change, and by the complete discharge of the colour on adding mercuric chloride. (The copper-sulphate test, either alone or combined with tincture of guaiacum, has not been found to be of any value for such small percentages.) By the above method differences of 0.002 per cent. can be detected without difficulty.

Bunting* has attempted to show that the ferric-chloride test as usually performed is practically valueless, for the following reasons:

I. That the colour reaction obtained with ferric chloride is much more reliable in an ether solution of sulphocyanate than in a water solution, so much so that an ether solution of dried saliva should always be made.

2. That other substances besides sulphocyanate may be present in the saliva which give a similar red reaction with ferric chloride.

I am not prepared to accept Dr. Bunting's theories.

I. Is the ethereal solution more reliable than the watery solution of potassium sulphocyanate? The following simple tests give the answer in the negative:

(a) Equal parts of sulphocyanate are dissolved in equal amounts of ether and water in separate tubes, the same amount of ferric-chloride solution is added to each, and a red colour of equal intensity develops in both.

(b) A water solution of sulphocyanate is made, an equal quantity of ether is added, and the whole well shaken. Ferric chloride is added, and only the lower or water solution takes a red colour-i.e., the ether had abstracted hardly any sulphocyanate.

(c) An ether solution of sulphocyanate is made, an equal quantity of water added, and the mixture well shaken. Ferric chloride is added, and the lower half (the water) is a deep claret red, whilst the upper (the ether) is only a light magenta-i.e., the water has abstracted nearly all the sulphocyanate from the ether.

(d) Similar results exactly are obtained if the ferric chloride be added before shaking the mixture, and the colour can be seen gradually passing from the ether to the water.

2. Is the possible presence in the saliva of other substances which give the ferric-chloride test a serious source of error? No, and for the following reasons :

The substance mentioned by Dr. Bunting is aceto-acetic acid. This acid is formed in the body in the course of diabetes only, and when it is present it is usually regarded as of very grave significance, and it is hardly likely that anyone would obtain the saliva of such a person for analysis without being aware of the general condition. Moreover, if it is present, the simple procedure of heating the saliva decomposes it and renders it negative to the ferric-chloride test.

Aceto-acetic acid is, too, very soluble in ether, and this lessens the value of the ether test.

Other substances which give the ferric-chloride test are the metabolized products of salicylic acid, carbolic acid, antipyrin, and thallin,

* Dental Cosmos, December, igio. 
and it is at present quite an assumption that these products are secreted by the salivary glands. Moreover, if it should be found that they are so secreted, it is a very simple thing to ascertain from the patient whether any such drugs are being taken.

Table IX. gives the sulphocyanate estimations obtained by the usual series of stimulations, the saliva from all the glands being collected in one receptacle.

\section{TABLE IX.}

Sulphocyanate Content of Saliva.

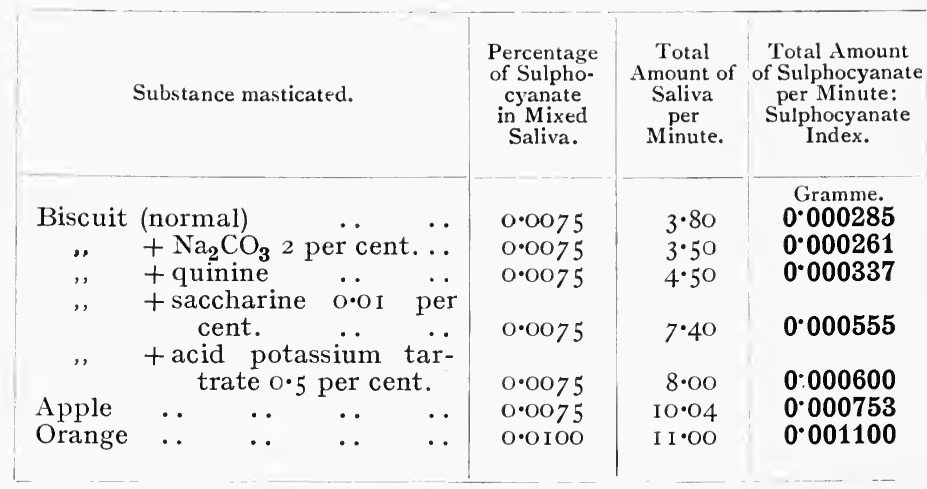

It is clear that the concentration of sulphocyanate is very little affected by the nature of the substance being eaten, the percentage composition being remarkably uniform with the exception of orange, which causes a rise of 0.0025 per cent. In this respect the sulphocyanate differs from the other constituents of saliva. Owing, however, to the increased or decreased amount of saliva per minute, the total amount of sulphocyanate in the mouth per minute varies greatly. Here also acids give the greatest amount, bitters and sweets the next greatest, whilst alkalies cause a diminution.

We have shown previously that sulphocyanate has an inhibitory effect upon lactic acid fermentation; if, therefore, it is wished to increase this effect, and not to diminish it, it is obvious that acids should be used and alkalies withheld. 


\section{Effect of Internal Administration.}

I. In Animals.-(a) A dog, weight 25 kilos, was given 6 milligrammes of pilocarpine by hypodermic injection. Ten minutes after the saliva was collected by aspiration from the mouth, and tests for sulphocyanate were entirely negative. 2.5 c.c. of I per cent. sulphocyanate were then given per os (this being three times the dose advocated by Greyger for children). Specimens of saliva were collected five, ten, forty-five, and seventy minutes afterwards, and in no case could even a trace of sulphocyanate be observed.

(b) A similar experiment was performed on a smaller dog, with corresponding doses. The results were exactly the same.

(c) A rabbit was given I milligramme of pilocarpine per kilo of body-weight hypodermically, and five minutes afterwards the saliva, upon being tested, gave a negative result for sulphocyanate. $0 \cdot 2$ gramme potassium sulphocyanate was then given, the rabbit was allowed to eat grass for ten minutes, and the mouth was swabbed out with distilled water. The saliva was then collected by aspiration, and showed sulphocyanate to be present to the extent of $0 \cdot 035$ per cent. Half an hour afterwards the percentage was the same, and in one and a half hours it was still 0.03 per cent.

2. In Man.-The following experiment illustrates the effect of the internal administration of potassium sulphocyanate in man:

Resting saliva contained (three estimations on three

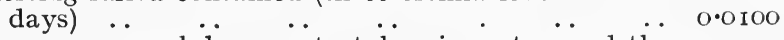

$0 \cdot 1$ gramme sulphocyanate taken in water, and the mouth well rinsed out-

5 minutes afterwards the saliva contained .. 0.0150

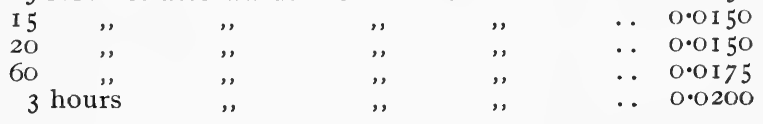


The percentage in resting saliva remained at $0 \cdot 02$ per cent. for seven days afterwards. It would not be correct, however, to say that this was due to the retention of sulphocyanate in the system; it may have been, or it may have been due to other unascertained causes.

There is some reason for thinking, however, that sulphocyanates are so retained and circulated between the stomach and the salivary glands. For instance, the abdomen of a rabbit whose saliva was negative for sulphocyanate was opened and the pylorus clamped. Five c.c. of a I per cent. potassium sulphocyanate were then injected into the stomach, and I milligramme of pilocarpine hypodermically into the lumbar region. In five minutes the saliva contained a trace of sulphocyanate, and in fifteen minutes the saliva gave a marked reaction.

A similar but more rapid result may be obtained by injecting potassium sulphocyanate hypodermically into the subcutaneous tissues.

It is therefore concluded that in man and some animals, but not in others, the amount of sulphocyanate in the saliva may be rapidly increased by internal administration; and that advantage of this may be taken in cases where a continuous anti-fermentative action is desirable, such as the rapid and acute caries of children.

The drug is not an active poison,* and may be given advantageously in repeated doses of $\frac{1}{4}$ to I grain.

* Ninety grains have killed a rabbit in four hours. There were convulsions in the case of a rabbit which died from $1 \frac{1}{2}$ drachms of a strong solution after ninety seconds. In other cases rabbits took 3 drachms without any injurious effects (Mayer-Tidy). The appearances after death were similar to those produced by the action of an irritant poison.

Westrumb (Tidy) found that 40 grains killed a dog in seven minutes, and another in two hours, convulsions being the prominent symptom, and the poison was found in the blood, lungs, liver, spleen, and kidneys.

Soemering found that 3 grains killed a dog in one minute. 


\section{Origin of Sulphocyanate.}

Undoubtedly the sulphocyanate in the saliva is a product of protein metabolism; but whether it is, as Fenwick* has suggested, to be regarded as a measure of the protein metabolism of the body seems not to be proved. An appreciable amount of protein in the form of mucin, epithelium, leucocytes, etc., is split up in the mouth into simpler compounds by the action of bacteria, and the question arises, Is sulphocyanate one of the compounds formed?

The following observations tend to show that this is the case:

(a) The percentage of sulphocyanate in mixed saliva from the mouth is always higher in the morning, before the teeth are cleaned, than it is shortly after cleaning and rinsing the mouth, the excess in the case of the author varying from 0.005 to 0.02 per cent., and in the case of another individual there was a difference of $0 \cdot 0$ I25 per cent.

(b) Mixed saliva collected from the glands direct contains a lower percentage of sulphocyanate than does the saliva from the mouth before or after such collection.

As instance the following series of estimations:

Per Cent. Teeth uncleaned for twenty-four hours .. Sulphccyanate $\left.=00^{\circ}\right)$ I 50 Saliva from glands direct (orange).. . . , $\quad=0 \cdot 0$ IOO Sal ." . (apple) . . " $\quad=0.0075$ Saliva from mouth immediately afterwards :, $=0 \cdot 0$ IOO Teeth cleaned and mouth rinsed . . . , , $\quad=0.0075$

(c) The amount of sulphocyanate in saliva increases upon incubation.

Saliva collected after cleaning the teeth and
rinsing the mouth Divided into two equal portions and incubated-
In 24 hours
$\begin{array}{llll}\cdots & \cdots & \cdots & \cdots \\ \cdots & \cdots & \ldots & \ldots\end{array}$
,$\quad=0 \cdot 0$ I $5 \mathrm{O}$
In 48

$\cdots$
$=0 \cdot 0$ I 75

* "The Saliva as a Test for Functional Disorders of the Liver," I 889 . 
(d) More sulphocyanate may be obtained from the precipitate of centrifuged saliva than from the clarified portion. (This, however, does not seem to be constant.)

(e) Traces of sulphocyanate may be developed in broth infected with mouth organisms.

It therefore would appear that in the bacterial metabolism of the precipitated organic constituents of saliva we have another active protective agency, and one which appears to be specially adapted to afford protection during the night when oral circulation is reduced to a minimum. I have noticed, too, on several occasions, that the organic constituents in saliva from immunes separate out much more quickly than they do in saliva from susceptibles.

Reference has been made to the associations between percentage of sulphocyanate and immunity to caries (see p. I98). In an examination made here of roo cases susceptible to caries the average amount of sulphocyanate present was 0.0043 per cent. This is distinctly low, and is much below the average for the few immune individuals whose saliva I have had the opportunity of examining. At the same time it is necessary to add that, contrary to the findings of Michel, Low, and Beach, there seemed to be no constant ratio between the amount of caries and the amount of sulphocyanate.

This may be gathered from the following typical examples:

\begin{tabular}{|c|c|c|}
\hline $\begin{array}{c}\text { Number of Teeth } \\
\text { esent }\end{array}$ & $\begin{array}{c}\text { Number of Teeth } \\
\text { carious. }\end{array}$ & $\begin{array}{c}\text { Percentage of Sulphocyanate in } \\
\text { Saliva. }\end{array}$ \\
\hline 24 & IO & $0 \cdot 0032$ \\
24 & IO & $0 \cdot 0026$ \\
24 & IO & $0 \cdot 0046$ \\
24 & I7 & $0 \cdot 0050$ \\
24 & 9 & $0 \cdot 007$ I \\
25 & 3 & $0 \cdot 0030$ \\
I2 & 2 & $0 \cdot 0055$ \\
28 & 28 & $0 \cdot 0050$ \\
\hline
\end{tabular}

It is curious that the healthiest mouth (European) and the most unhealthy that I examined both showed the same 
percentage of sulphocyanate in the saliva-namely, 0.005 per cent. The one individual had thirty-two perfect teeth, the other thirty-two septic teeth.

In addition to the above, I have examined eleven cases in which either sulphocyanate was absent, or only so faint a trace of it present, that it could not be estimated colorimetrically. All these were susceptible to caries, but not more so than other cases which showed a fair percentage of sulphocyanate in the saliva.

The following figures compare immune with susceptible individuals, and it will be noticed that the saliva of immunes varies just as does that of susceptibles with regard to the sulphocyanate content.

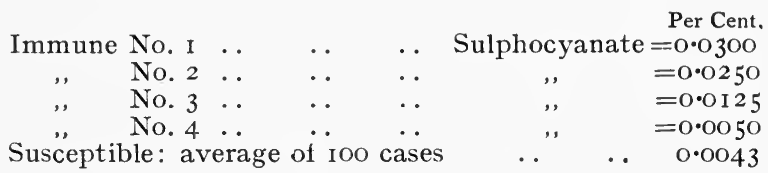

Waugh* has claimed that sulphocyanate has the property of preventing organisms from forming adhesive plaques. His experiments are, however, somewhat inconclusive, since ten tubes of broth to which sulphocyanate had been added, infected with mouth organisms, showed no plaque formation, but of ten control tubes only one showed plaque formation. The addition of carbolic and cassia to the broth caused an increase in plaque formation, but when sulphocyanate was also added this was slightly decreased.

\section{Other Possible Effects of Sulphocyanate in Saliva.}

There are besides an anti-fermentative effect three other ways in which sulphocyanate in saliva may manifest an action; these have been briefly investigated.

r. Does it restrain the action of ptyalin? I have not been 
able to find that this is so. Experimentally doubling the amount of sulphocyanate in any saliva does not seem to reduce the digestion of starch at all.

2. Does it retard the peptonizing effect of organisms similarly to their acid-production? Investigations so far tend to show the reverse.

In these experiments saliva was obtained and ammonium sulphocyanide added to different portions in varying quantities. The peptonizing power of the saliva was then estimated by the Mett's tube method, dilute gelatine being used for fourteen days at room temperature. The following figures express the result:

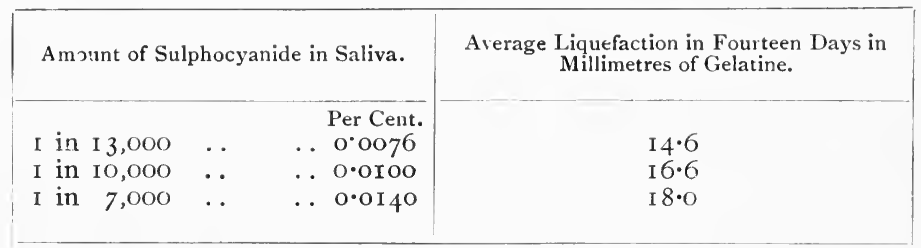

3. The Proliferation of Organisms.-A number of cultures from saliva have been made in Petrie dishes on agar containing $0.05,0.04,0.02,0.01$, and 0.005 per cent. sulphocyanate. These experiments have been repeated several times, but I have been unable to find that the addition of the sulphocyanate to the agar made any appreciable difference to the growth and number of the colonies which developed, except, perhaps, that the colonies of the Streptococcus brevis appeared to be somewhat retarded. On the other hand, colonies of Staphylococcus albus appeared to grow better in the 0.05 per cent. sulphocyanate than in the o.or per cent. Similar experiments were done, glucose agar being used, with neutral red added, to see whether the acid-forming colonies might not be specially retarded; but I was unable to find that this was so (see Figs. 65 and 66).

Conclusion.-On the whole, we may conclude that whilst sulphocyanate may be a beneficial element in saliva, and one 


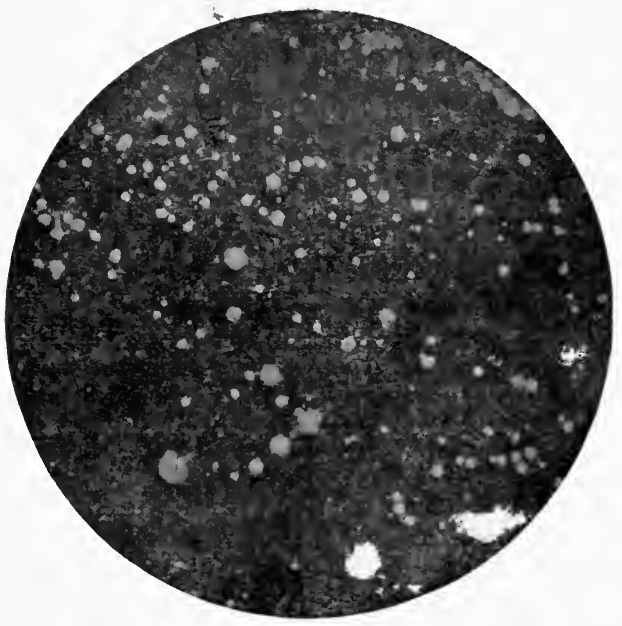

Fig. 65.-Colonies developed in Forty-Eight fiours on Agar containing 0.05 per Cent. Potassium Sulphocyanate.

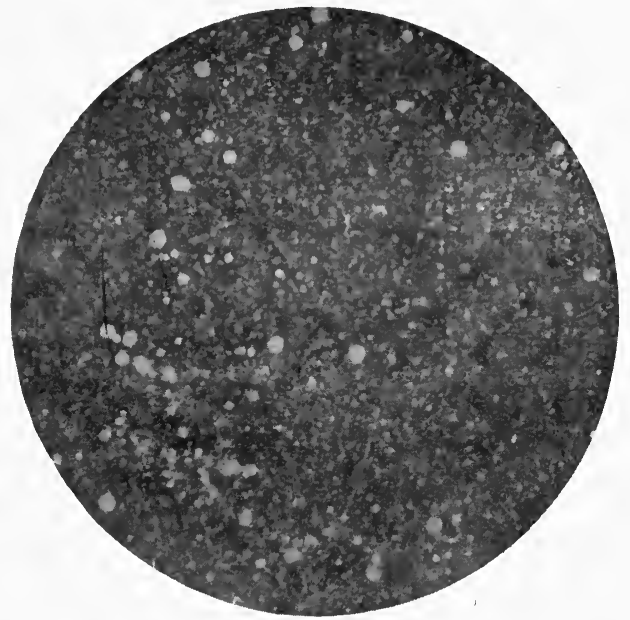

Fig. 66.-Colonies developed in Forty-Eight Hours on Agar containing o.oi per Cent. Potassium Sulphocyanate. 
making for freedom from disease, yet it cannot be regarded as the most important, much less as the only, factor in producing a natural immunity to dental caries or oral sepsis.

\section{Phosphates.}

There can be no doubt but that the phosphates of the saliva have a protective action upon the teeth. Lactic acid readily combines with phosphates to form harmless lactates, and the lactic acid will be more readily and rapidly " satisfied " if the phosphates are in solution, than if they are in a solid and extrenely hard mass, like enamel. Acid phosphates may be formed, but there is no evidence to show that these, under such circumstances, can exert any effect on the enamel surface.

Reference has also been made to another probable protective function of phosphates, in penetrating and hardening the outer strata of enamel after eruption. Therefore the phosphate content of saliva must be of importance in considering the matter of immunity to caries. The question then arises, Are the phosphates subject to the same variations as the alkaline salts, and can they be increased or decreased at will ? In order to determine this point, the phosphates present in the saliva obtained by various stimulants were estimated. (The method adopted was that of titration with $\frac{\mathrm{N}}{5}$ uranium nitrate. The saliva was diluted, and there was added a small quantity of sodium acetate acidified with acetic acid. The indicator used was potassium ferrocyanide.)

Table $\mathrm{X}$. gives the results of such estimations. The saliva was collected from the glands direct during mastication, and both tubes were run into one receptacle.

It will be observed that both the amount per c.c. and the amount per minute vary considerably according to the substance being eaten. Again, the addition of an alkali causes a diminution; sodium chloride slightly increases the amount, 
and so does a bitter flavour, but not to the extent that an acidsweet taste does; and a pure acid flavour, like tartaric acid is more efficacious still. "Natural" excitants, however, like apple and orange, here also produce the greatest amount of the salts per c.c.; and since these also produce a great increase in the total amount of saliva, the increase in the total phosphates per minute is still more marked. It is seen that

\section{TABLE $X$.}

Phosphate Content of Saliva.

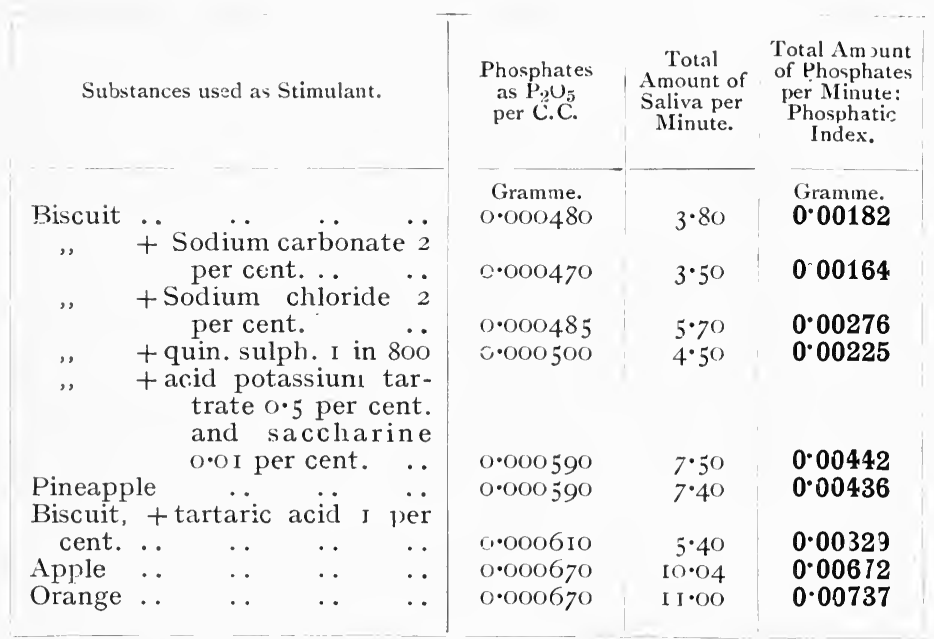

the addition of sodium chloride increases the total phosphates per minute more than double; that apple and orange treble and quadruple the amount respectively.

\section{Chlorides.}

The function of sodium and potassium chlorides in saliva is probably largely protective to the mucous membrane of the mouth; and further they, undoubtedly, increase the sapidity of ordinary articles of diet, and thus induce a further reflex 
flow of saliva. It is well known, for instance, that washing the mouth out with a weak solution of common salt increases the perception of other substances-such as sweets, and also that the presence of sodium chloride in saliva materially assists in holding the calcium phosphate in solution.

Estimations of the amount of chlorides present-as sodium chloride-in the saliva under various conditions have been made, and the results tabulated in Table XI.

\section{TABLE XI.}

Chiorides. (Estimated as Sodium Chloride.)

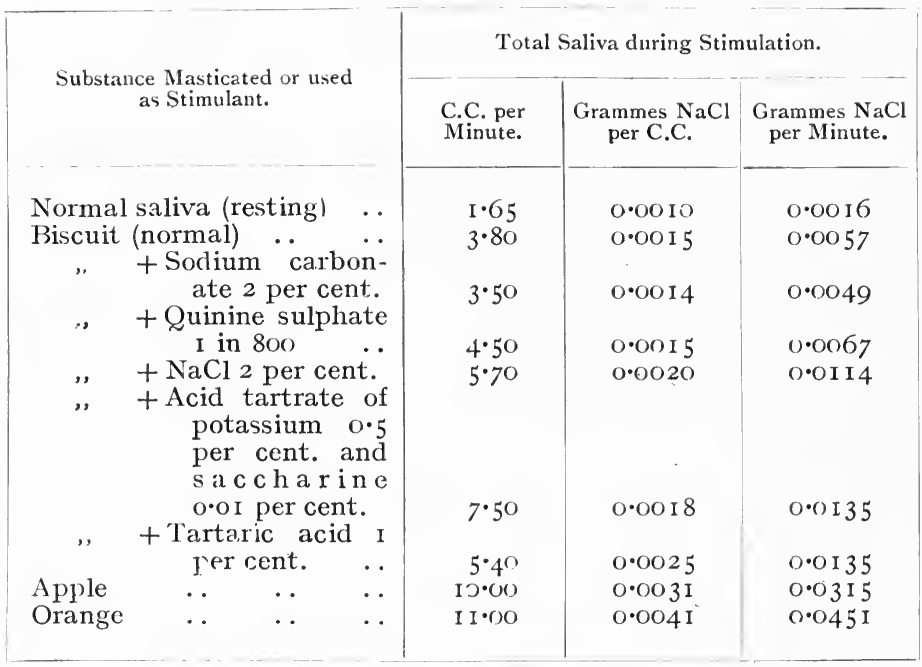

The method used was as follows:

About Io c.c. of mixed saliva were collected with the segregator and cannula, the mucin removed by precipitation with acetic acid. One c.c. of nitric acid and a known volume of standard silver nitrate were then added; the silver chloride was removed by filtration, and the amount of free silver nitrate remaining in the filtrate was estimated by titration with a standard solution of ammonium sulphocyanide. By 
deducting this from the amount of silver nitrate originally used, the amount used in combining with the chlorides is obtained, and from this the weight of the chlorides, as sodium chloride, is readily calculated. It is seen upon reference to the table that the amount of chlorides present under active stimulation conditions is in excess of what has been found for other salts-both in amounts per c.c. and per minute; for various stimulations it follows closely the order obtained for other constituents. The acid-flavoured biscuit and acid fruits give the highest amounts per c.c. and per minute; whilst again the alkaline biscuit gives a subnormal amount both per c.c. and per minute. The sodium chloride biscuit increases the amount per minute by seven times. It was thought that possibly to this form of stimulus the chloride content might not have been raised, but that evidently is not so; in fact, a greater response is obtained than is the case for either alkaline salts or phosphates.

In addition to the sedative and protective effect of the chlorides upon the mucous membrane of the mouth, doubtless the marked increase and excess of chlorides over other salts, and especially over the alkaline salts, serves the very necessary purpose of counteracting the depressant effect which an increase of the latter by themselves would be bound to bring about on the gustatory nerves.

For instance, the alkaline salts for biscuit and orange stimulations are as I : 5.5 ( $c f$. Tables I. and II.); but the chlorides for the same stimulations are as $I: 7 \cdot 9$. Thus the effect of an increased stimulus tends not to be automatically cut off when the excitant is removed, but rather to be perpetuated.

\section{Mucin.}

The amount of mucin present in saliva is not of so much importance as the condition in which it is present.

In the scanty and viscous condition in which it is frequently 
present, it is undoubtedly to be regarded as being favourable to the development of dental caries and oral sepsis, in so far as it forms a sticky mixture with carbohydrates, binds them to the teeth and tissues, and thus facilitates the prolonged action of the incorporated organisms. It has been suggested that mucin is a source of mucic acid, which may attack the enamel. Cook,* however, has shown that this conversion into mucic acid can only occur under aseptic conditions, which certainly cannot obtain in the mouth. Mucin by itself in the presence of mouth organisms undergoes an alkaline decomposition, and is therefore to be regarded as a protective agent. This I have repeatedly observed, and it may be demonstrated in the following manner. Saliva is collected, and the mucin precipitated with dilute acetic acid and filtered. The precipitate is dissolved with warm sodium hydrate, litmus is added, and the solution just brought to the neutral point by the addition of acid. The solution is now infected with mouth organisms and incubated, an alkaline reaction rapidly develops, and in two days may be equal to as much as 2.7 units $\left(\frac{\mathrm{N}}{50} \mathrm{H}_{2} \mathrm{SO}_{4}\right)$ per c.c. This is much more likely to take place in the mouth, and the resulting alkalinity to be greater and more efficacious, in the case of mucin precipitated in a solid state on or around the teeth. Such precipitation is produced by all weak acids and by acid salts, notably by acid potassium tartrate, $\uparrow$ and flakes of solid mucin rapidly separate out and cling to any solid substance. This undoubtedly is, I think, the source of some of the " plaques" found upon teeth. Such plaques would, of course, be subsequently dissolved by the increased flow of highly alkaline saliva.

Mucin is also precipitated from stagnant saliva to a lesser extent, and in a less solid form, upon the escape of carbon

* Johnson: "Operative Dentistry," p. 477.

$\dagger$ Kirk has also noted that mucin may be precipitated by acid sodium phosphate. 
dioxide and the consequent precipitation of some of the alkaline salts; and where the salivary secretion is deficient the viscous mucin clinging to the teeth does not tend to be redissolved on account of the decreased alkalinity of the saliva. This, I think, probably explains the difference between the different kinds of "plaques" observed. The ones which are " actively" precipitated by acids are firmer and more compact; those " passively" precipitated by stagnation are softer and more viscous, or " gelatinous."

When the mucin is precipitated by acids or acid salts, alkaline salts are also carried down incorporated with it, and it is almost impossible to render the flakes of mucin acid even by the addition of large amounts of acid. The teeth in this way become coated with an organic alkaline film, which acids have the greatest difficulty in penetrating. On the other hand, if fermentable carbohydrates are incorporated in the mucin, the organisms attack these in preference to the mucin, and thus an acid reaction develops until all the carbohydrate is used up.

Another advantage derived from the active precipitation of mucin is the elimination of a strong non-electrolyte from the saliva, thus facilitating dialysis of its inorganic salts. There is manifested also another beneficial action of mucin when acid substances are being masticated. All the particles seem to be more closely bound together - united to the central bolus; so that, when the latter is swallowed, practically all the particles follow and do not remain isolated and adherent to the teeth, as happens with such articles as bread, biscuits, or "sweets."

The association observed clinically between ropy saliva and acute caries in children may be due to one or a combination of all of three causes: (I) It may be a special characteristic of chronic pain associated with a "nervous" or mental effect; (2) it may be due to the original cause of the caries$i$ e., a too liberal consumption of "free" sugar; (3) it may 
result from a deficient alkalinity of the saliva. It may, further, possibly be regarded as an effort by Nature to provide a material which, if precipitated, would undoubtedly protect painful surfaces against acid stimuli.

A few estimations of the amount of mucin present under various conditions have been made, but the technical difficulties are considerable, and a satisfactory method for all substances has not yet been found.

The difficulties arise from the fact that about 80 per cent. of the mucin in mixed saliva comes from the buccal mucosa, and hence is very difficult to collect. This has been attempted by masticating substances, expectorating the bolus,

\section{TABLE XII.}

mucin Content of Saliva.

\begin{tabular}{|c|c|c|c|c|c|c|c|}
\hline \multicolumn{5}{|c|}{ Substance used as Stimulant. } & \multirow{2}{*}{$\begin{array}{l}\text { From Glands } \\
\text { alone. } \\
\text { Grammes \% } \\
\\
-\end{array}$} & \multirow{2}{*}{$\begin{array}{c}\begin{array}{c}\text { From whole } \\
\text { Mouth. } \\
\text { Grammes \% }\end{array} \\
0.23\end{array}$} & \multirow{2}{*}{$\begin{array}{c}\text { Difference= } \\
\text { from Buccal } \\
\text { Mucosa. }\end{array}$} \\
\hline Normal & sal & res & . & . & & & \\
\hline Apple & .. & .. & $\ldots$ & .. & $0 \cdot 025$ & $0 \cdot 207$ & O• I 82 \\
\hline Orange & . & .. & $\ldots$ & .. & $0 \cdot 03 \mathrm{I}$ & $0 \cdot 25$ & $0 \cdot 219$ \\
\hline Biscuit & $\cdots$ & $\cdots$ & $\cdots$ & $\cdots$ & $0 \cdot 029$ & 0.27 & $0 \cdot 24 \mathrm{I}$ \\
\hline Sugar & .. & . & . & . & $0 \cdot 135$ & $0 \cdot 69$ & $0 \cdot 555$ \\
\hline
\end{tabular}

and filtering through muslin. Ten c.c. of the filtrate were then taken, mixed with a large excess of 0.5 per cent. sodiumhydrate solution, allowed to stand for some time in order to dissolve the mucin, and again filtered (through paper filters); acetic acid was added to precipitate the mucin, and the latter collected on a dried and weighed filter-paper. The precipitate was then washed with 2 per cent. acetic acid, alcohol, and ether, and the filter-paper again dried and weighed. The mucin from saliva collected by expectoration immediately after mastication has also been similarly estimated, and the results in the two cases were so close that the average of the two is given in Table XII, 
In the case of apple, orange, biscuit, and sugar (cane), it is seen that the amount of mucin from the whole mouth is greatly in excess of what is obtained from the salivary glands direct; the difference between these two gives the amount derived from the buccal mucosa. There is not a great deal of difference between the several amounts for biscuit, apple, and orange, but sugar evidently is a much more powerful excitant of the mucous glands than weak acid substances; roughly, three times the amount being secreted. Sugar and biscuit, of course, do not precipitate the mucin at all, but, on the contrary, form with it an exceedingly sticky and viscous mass. This will be referred to again later, but is strikingly exemplified when one attempts to filter masticated mixtures of biscuit and saliva, and apple and saliva, for instance. The saliva from the apple mixture passes through the filter readily, but from the biscuit or sugar mixture filtration is either exceedingly slow or does not occur at all. Similar conditions, of course, obtain in the

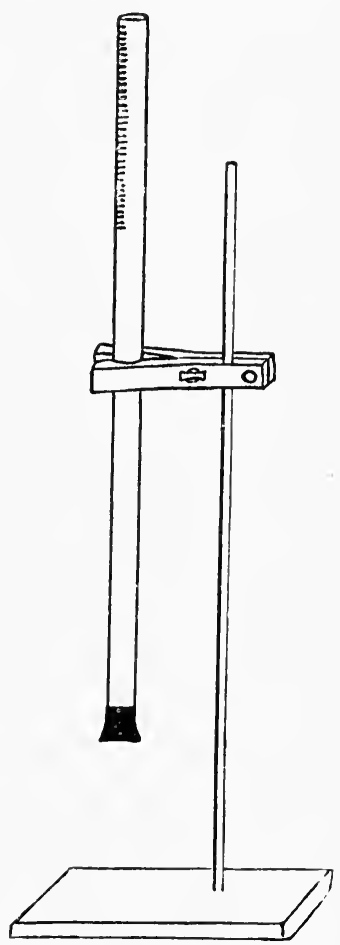

FIG. 67.-MUU C INOMETER (FOR ApProximate EstiMATIONS ONLY). mouth, where the crevices between the teeth may be regarded as the filter.

The constant consumption of "sugar" must therefore, by continual stimulation, cause a hypertrophy of the mucous glands, and give rise to a higher mucinous secretion in the mouth, and doubtless accounts to some extent for the hyperæmic and 
swollen condition of the mucous membrane so frequently seen in people who consume much "sugar" in its artificial forms.

For the estimation of mucin for clinical purposes I use a long glass tube, graduated at the upper end, and with a cork in the lower end (see Fig. 67). The saliva is poured in, and 20 per cent. acetic acid is added until the level of the graduations is reached. The mucin is rapidly precipitated and rises to the top; on standing, the precipitate condenses, and the amount may be read off in one hour. Each division on the tube equals approximately 0.002 gramme of mucin. This is not absolutely exact, however, since a small but variable amount of epithelial débris and carbon dioxide gas is usually included amongst the precipitated flakes of mucin.

\section{Viscosity of Saliva.}

Time has not permitted much work to be done yet on this subject. That saliva varies in viscosity has long been recognized, and it has been customary to associate viscous or ropy saliva with extensive caries; but this is a point which is by no means proved, and the association, when observed, is open to several other explanations. Absolute viscosity is a somewhat tedious matter to measure with accuracy. For the present purpose, however, it is not so much the absolute as the comparative viscosity with which we are concerned. I therefore adopted the method employed by Sellheimnamely, measuring accurately the time which a given quantity of saliva takes to pass through a narrow glass tube; 3 c.c. of saliva were used, and the glass tube was I c.c. in length and 0.5 millimetre in diameter, and the experiments were done at $35^{\circ} \mathrm{C}$. In this case, however, the results are expressed in terms of water, thus:

Viscosity of saliva $=\frac{\text { Time in seconds for passage of I c.c. of saliva. }}{\text { Timeinsecondsfor passage of I c.c. of distilled water }}$

$$
\text { Or, } \mathrm{V}=\frac{\mathrm{T}_{1}}{\mathrm{~T}_{2}}
$$


The viscosity of the saliva was measured five minutes after eating various substances, with the following results:

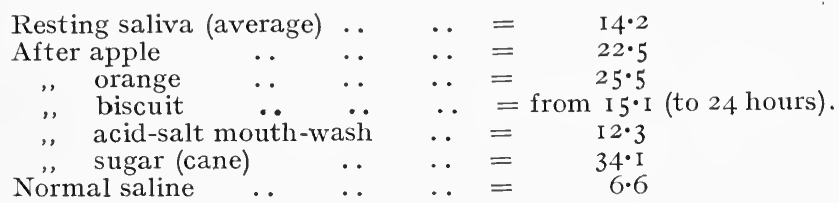

These observations show, then, that cane-sugar produces the most viscous saliva, and salt in solution the least viscous; acids are intermediate. Biscuits, apparently, do not affect actual viscosity very much; but in practice, owing to there always being small particles of detritus present in the saliva, the tube frequently blocks, and thus the effect is produced of extreme viscosity.

The question as to whether a viscous saliva is harmful or not is not an easy one to decide. It is commonly supposed that "ropy" saliva is associated with advanced caries. But the question arises, Is " ropy" saliva of necessity more viscous? W. D. Miller, speaking on this point, said: "I am inclined to think that the importance of the viscosity of saliva has been overrated. I have tested a great many cases, and have not found, on the whole, that the viscosity has had a very great influence upon the amount of caries present. I have had a number of cases in which the saliva was so viscous that, in attempting to pour it from one vessel to another, it would go over in a clump, like the white of an egg, and yet the patient was absolutely immune from caries. Also, I have seen saliva as thin as water, and yet the teeth were going to destruction." "*

My experience has been exactly similar. The least viscous saliva that I have seen (4.I) was in a patient whose every tooth was carious and mouth extremely septic. On the other hand, in another individual (European) who was perfectly immune

* Dental Cosmos, 1905, p. go. 
to caries, the saliva would not spot from the tube at all, but ran slowly in one long viscous thread. This is a subject which requires more investigation. That a certain amount of viscosity is beneficial and desirable there is no doubt, and especially is this so after a meal of highly refined carbohydrates. It is in this way that fruit exerts a great deal of its beneficial action; through its moderately raising the viscosity the débris is collected, held together, and swallowed. A saliva of low viscosity would flow over the débris, and per-

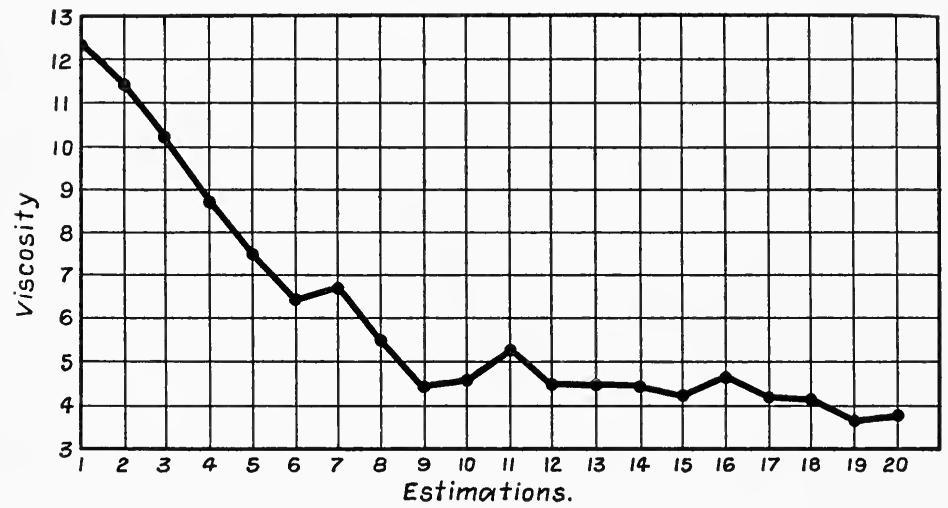

Fig. 68.-Curve showing Reduction in Viscosity of Saliva PRoDUCED BY REPEATEDLY PASSING IT THROUGH A NARROW TUBE, the Temperature being Constant.

haps neutralize or slowly dissolve it, but it would not be removed en masse. A highly viscous saliva, on the other hand, would be equally disadvantageous, in that it might tend to bind particles to the teeth; especially is this the case with cane-sugar, which forms a definite compound with the mucin and which undergoes a slow acid fermentation. The question, too, is largely related to the rate of flow. A saliva of viscosity, say, 25 might be highly dangerous with a flow of only 0.5 c.c. per minute; but with a flow of $\mathrm{I} \cdot 5$ or 2 c.c. per minute it would exert a very decided cleansing action-more so, for instance, 
after biscuits had been eaten than a saliva of a viscosity of Io only.

Again, it would seem desirable that we should obtain this viscous action at the termination of a meal; but through "inter-meal" times a saliva of lower viscosity might be better. This may be obtained in two ways: first, by washing the mouth out with normal saline, which immediately reduces viscosity by one-half; or, secondly, by forcing the saliva through the interdental spaces several times.

The reduction in viscosity produced by manipulation is shown in the accompanying chart. The above observations further indicate a method of controlling the viscosity of saliva. If, upon testing, it is found to be normally too viscous, saline is indicated; if the viscosity is much too low, then acids may be used alone.

It would seem that saline would be useful in those cases where caries is caused by fluid carbohydrates, but that acids would be indicated for those cases where caries is caused by starchy débris.

\section{Salivary Stimulants not Previously Considered.}

Effect of Pyrethrum.-It is well recognized that pyrethrum has a stimulating effect upon the salivary glands, and this investigation would not be complete without a reference to its action.

Its effect on the secretion of saliva has been tested by a similar method to that used for other substances, but, as will be readily seen from the figures, its power as a salivary stimulant cannot compare with that of acids.

A biscuit was treated with a 2 per cent. mixture of tincture of pyrethrum and water, and the saliva collected during mastication, with the following results as regards amount and alkalinity: 


\begin{tabular}{|c|c|c|c|c|c|}
\hline & & & Parotid. & $\begin{array}{c}\text { Submaxillary } \\
\text { and } \\
\text { Sublingual. }\end{array}$ & $\begin{array}{l}\text { Total from } \\
\text { all Glands. }\end{array}$ \\
\hline $\begin{array}{l}\text { Amount per minute } \\
\text { Alkalinity per c.c. } \\
\text { Alkalinity per minute }\end{array}$ & $\begin{array}{l}\cdots \\
\cdots \\
\cdots\end{array}$ & $\begin{array}{l}\cdots \\
\cdots \\
\cdots\end{array}$ & $\begin{array}{r}0 \cdot 15 \\
1 \cdot 60 \\
-\end{array}$ & $\begin{array}{c}3 \cdot 30 \\
0 \cdot 68 \\
-\end{array}$ & $\begin{array}{l}6 \cdot 90 \\
0 \cdot 72 \\
4 \cdot 96\end{array}$ \\
\hline
\end{tabular}

Comparing this with similar stimulations given in Table II., we see that pyrethrum used in this manner ranks very little higher than oil of cloves, and is not so efficacious as sodium chloride. The low alkalinity of the submaxillary saliva is particularly noticeable.

The solution of pyrethrum used was much stronger than could be used with comfort by itself, but combined with the biscuit its effect was less marked. The mouth was washed out for one minute with Io c.c. of a I per cent. mixture of tincture of pyrethrum and water, this being the maximum strength which can be used without disagreeable effects. Five minutes afterwards the saliva was collected by expectoration, and showed a total secretion of $2 \cdot 2$ c.c. per minute, and an alkalinity index of $2 \cdot 28$ - a definite increase over the " normal resting," but not a very marked one, and not so great as that produced by acid mouth-washes, as will be shoẉn subsequently (see p. 279).

Pyrethrum also produces a greater secretion of mucin, probably by its obvious stimulating effect upon the buccal mucous membrane; and since this mucin is not precipitated, it must be regarded with disfavour. After washing the mouth out with the I per cent. mixture of tincture of pyrethrum and water, the saliva contained $0 \cdot 29$ per cent. of mucin.

Pyrethrum in strong solutions is certainly a powerful salivary stimulant, but it is then without the range of practicability, since certainly no one could be persuaded to use it, owing to its prolonged and extremely disagreeable effect on the tongue and oral mucous membrane. 
As a therapeutic agent in special cases it may be useful, but it cannot be recommended either as a flavouring agent for food or as a routine prophylactic.

The Stimulus of Pain.-The effect upon the salivary glands of painful sensations within the mouth is known to be stimulating. The question has not been properly investigated, but probably both the situation and the character of the pain have definite effects upon the amount and composition of the resulting secretion. The following is an analysis of mixed saliva obtained by aspiration during the operative treatment of a lower premolar:

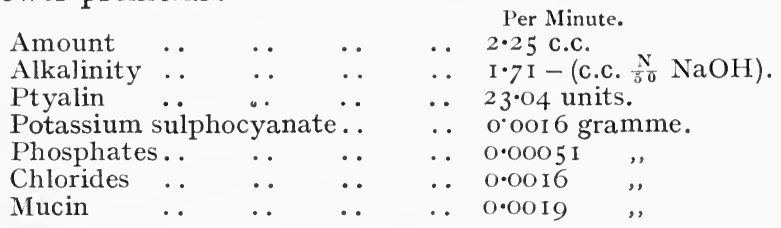

It is therefore chiefly a watery saliva, both organic and inorganic constituents being markedly low.

\section{The Effect of Alkalies and Acids on the Dialysis of Saliva.}

We have before alluded to the probable function of Nasmyth's membrane and its action as a dialyzing membrane, and therefore the important rôle it plays in the hardening of the superficial layers of enamel after eruption. We have also shown how various substances promote a flow of saliva of varying character, and have noted the effects of various chemical substances upon the constituents of saliva, amongst the most important being the fact that alkalies added to saliva cause a precipitation of the phosphates and a retention of the mucin in solution, whilst acids have an exactly reverse effect. If this be so, the addition of an alkali to saliva should materially reduce the amount of phosphates that would dialyze through Nasmyth's membrane, but the addition of acids would increase the amount. 
The following is experimental evidence that such action is possible and extremely probable:

Thirty c.c. of mixed saliva were taken and divided into three parts-A, B, and C. To A nothing was added, and it acted as a control; to $\mathrm{B}$ was added 0.04 gramme of sodium carbonate, and to $\mathrm{C}$ a similar amount of acid potassium tartrate. Dialyzers (the membrane being of sheep's intestine) were then placed in each, and filtration avoided by means of 0.45 per cent. sodium chloride inside the dialyzers. Dialysis was allowed to proceed for five days, and the amount of phosphates which had passed through the membrane in each case estimated. The following table expresses the result:

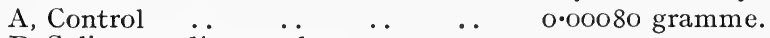

Amount of Phosphates as $\mathrm{P}_{2} \mathrm{O}_{5}$ dialyzed in Five Days.

$\mathrm{B}$, Saliva+ sodium carbonate $\quad$. 0.00025 ",

C, Saliva + acid potassium tartrate o.0O1 30 ",

Each ro c.c. of saliva originally contained

It is observed from this that the addition of the alkali has diminished the normal amount of phosphate which should dialyze by more than two-thirds, and that the addition of the acid salt has increased it by more than one and a half times; also that, approximately, in the control one-seventh of the total phosphates have dialyzed, in the alkali saliva only one-twentieth, but in the tartrate saliva one-fourth of the whole have passed across. The latter result is due, of course, to the phosphates being held in solution instead of being more or less precipitated, as in $\mathrm{A}$ and $\mathrm{B}$, and also to the elimination of the non-electrolyte mucin by its precipitation.

Although membranes differ in their dialyzing powers-and Nasmyth's membrane may differ in this respect from sheep's intestine-there is no reason to doubt that the relative proportions for the same salts would be the same in each. 


\section{The Possible Presence of Immune Bodies.}

This subject was investigated by Miller, * who came to the conclusion that the protective bodies of the normal bloodserum do not under normal conditions pass into the saliva in sufficient quantity to be detected by ordinary means. Yet there is some evidence that "immune" bodies do pass into the saliva. For instance, it is known that snake-charmers, by allowing themselves to be bitten by snakes of increasing size, acquire a high degree of immunity to the snake poison; and, further, that the saliva of such persons applied to the bite of a snake in another person has a beneficial action.

Secondly, it is known that in acute diseases, like diphtheria, cholera, and pneumonia, the saliva exerts a deleterious effect upon the particular organisms concerned. Therefore it would seem to be proved that it is possible for immune bodies, or anti-bodies, to be present in the saliva.

The question then arises as to whether there are any such bodies present in the saliva of persons immune to dental caries, and whether they are present in sufficient quantity to account for the immunity. The importance of " opsonins " in the blood as a factor in immunity has of recent years been widely recognized, and there seems no reason why opsonins should not pass to some extent into the saliva, where they might exert a deterrent effect upon the development of the mouth organisms.

Estimations of such action in the saliva have been commenced, working along the ordinary opsonic-index methods. Leucocytes are obtained by centrifuging blood, and washed in the usual manner. About 2 c.c. of the saliva to be examined are then taken and centrifuged for three or four minutes; the epithelial débris and some of the mucin will be deposited, leaving an opalescent upper portion, which

* Dental Cosmos, I903, p. I. 
still contains a large number and variety of organisms. This cleared saliva is taken as representing both the bacterial emulsion and the fluid (serum) containing the opsonin. Equal volumes are then obtained of cleared saliva and washed leucocytes, and after being well mixed the whole is incubated for fifteen minutes. Smears are then made in the usual manner, stained with Wright's stain, and the number of organisms taken up by the leucocytes counted.

The method has very obvious imperfections and difficulties, not the least of which is the fact that saliva is not isotonic with blood-serum, and hence the corpuscles are distorted and sometimes broken up. Attempts to make the saliva isotonic by the addition of solution of sodium chloride have not been very successful, since, as has already been shown, the saliva varies so much in composition. The mucin in the saliva, there is reason to believe, also interferes with the phagocytic action of the leucocytes.

A fair number of " counts" have been made, chiefly with the saliva of three individuals, one of whom (A) is nearly immune to caries (i.e., aged twenty-five, and having only one or two small carious cavities previously filled), one (B) is susceptible to a slight degree, and the other (C) is susceptible to about the average degree. In all of these phagocytosis on every occasion was extremely feeble, although there were always plenty of organisms present.

Organisms and leucocytes are frequently seen in close proximity, yet not one organism may be absorbed by the leucocytes. Again, when phagocytosis does occur, it does so very irregularly.

The results of these estimations are as follow:

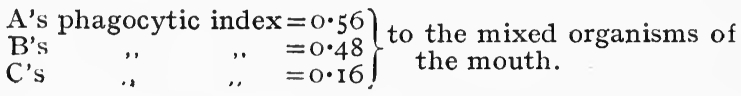


One could not from these calculate an "opsonic index" for the various salivas. It does not even prove, although it suggests, that the increased phagocytosis of $\mathrm{A}$ was due to the presence of opsonins in the saliva; the increase might have been due to a difference in the chemical or physical nature of the saliva. It does indicate, however, that there is either the presence or absence of some substance in the saliva of a relatively immune individual which facilitates phagocytosis.

Phagocytosis, however, does not occur normally in the saliva to any appreciable extent. It is well known that "altered" leucocytes are normally found in saliva, and the fact of their being altered is due, most probably, to the saliva being hypotonic to blood-serum. An examination of the deposit in centrifuged saliva stained with Wright's stain reveals many such leucocytes, but I have not been able to satisfy myself of any evidence of active phagocytosis (in mouths free from suppuration).

\section{Epithelial Débris.}

Mixed saliva from the mouth always contains large numbers of squamous epithelial cells, and, in marked contrast to the leucocytes, these are always loaded with organisms. Fig. 69 shows such a cell crowded with organisms, and two leucocytes also (slightly out of focus) which contained no organisms. The organisms are, of course, merely lying on the epithelial cells; but whether the saliva be centrifuged or not, by far the greater number of organisms present in any film are always attached to an epithelial cell. It does not seem at all likely that these are only the organisms which grew against that particular cell before it desquamated; it would seem to be far more probable that there is some attraction, simply physical, or maybe " vital," by which the organisms are collected on the cell. At any rate, there can be no doubt that these cells act as carriers of organisms, and that as a rule they 
carry them to destruction in the hydrochloric acid of the stomach. In this manner they must rank as one of the chief factors in keeping down the number of organisms in the mouth. Therefore articles of diet which are "demulcent" in character tend to prevent the exercise of this function, as also do other articles, such as pure cane-sugar, which excite a strong secretion from the mucous glands, and so by preventing friction lessen desquamation.

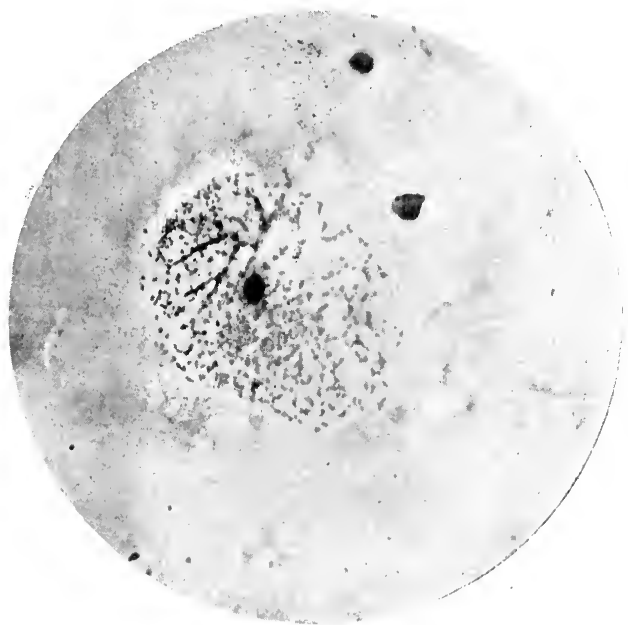

Fig. 69.-Epithelial Cell from the Saliva loaded with ORGANISMS.

If the epithelial cells are not swallowed, they are digested by the organisms upon them, and thereby alkaline decomposition products are formed.

\section{Antiseptic Properties of Saliva.}

Miller* held that those constituents of human saliva which have antiseptic properties are present in too small quantities to in any way impede the development of bacteria.

* "Micro-Organisms of the Human Mouth," p. 4I. 
Sanarelli* found that saliva filtered through a PasteurChamberland filter possessed the power of inhibiting the growth in vitro of Staphylococcus aureus if the latter was only present in small quantities. The inhibition, however, was inversely proportional to the number of organisms present, and pneumococci were unaffected.

I think that undoubtedly saliva has a mild antiseptic; action, but that this action is only exerted on those organismis which are introduced freshly into it. If, on their being introduced, the amount of antiseptic power is insufficient-i.e., the saliva being viscous or scanty--to destroy the organisms, then they soon become tolerant of saliva and acclimatized to oral environment.

\section{Abnormal Constituents.}

Urea, ammonia, lactic acid, leucine, traces of bile acids, bile pigments, glucose, and glycogen, have all been described as present occasionally in the saliva. $\dagger$ These, when present, have only been found in minute quantities, and most of them from the present point of view are unimportant.

The two carbohydrates, however, might be of extreme importance. The presence of glycogen has been confirmed by Michaels $\ddagger$ in some individuals in considerable quantities.

I have no evidence to disprove this statement; but, if we bear in mind the fact that glycogen is rapidly converted into maltose and isomaltose by ptyalin, it is a little difficult to conceive how the two could be present together, secreted by the same glands. They are physiologically incompatible. There are two possible explanations: (I) That ptyalin is absent entirely from the saliva of those persons in whom glycogen is said to be present. (2) That the glycogen is present by con-

* Quoted by Goadby, op. cit., p. 94.

† Gamgee: "Physiological Chemistry"; Schäfer: "Textbook of Physiology."

‡ "Sialo-Semeiology" Paris, Igoo. 
tamination with the mouth, since it is found in pus, in pathological growths, and in fungi.* In order for one to be absolutely sure of its presence in the saliva, the latter would need to be collected from the glands direct.

As regards glucose, some observers state that this is present in the saliva in cases of diabetes, $\uparrow$ whilst others deny this. $\ddagger$ Carlson and Ryan $\S$ have shown that traces of glucose are normally present in the saliva of the cat, but failed to demonstrate its presence in man in a case of diabetes.

I have examined the saliva for glucose in a number of healthy and sick persons, but on no occasion has even a sign of it been present. The diseases from which the patients were suffering were: diabetes, pronounced and mild; nephritis, acute and chronic; Graves' disease; typhoid fever; phthisis; gastro-enteritis; anæmia; oral sepsis (gastritis and fleeting joint pains); neurasthenia; cystitis; aneurysm; locomotor ataxy, and syphilis. There was, of course, no reason to suppose, except in the diabetes cases, that the saliva would con tain glucose; but in view of the fact that these patients all exhibited more or less oral sepsis, it is important to be able to show that the primary source of the lactic acid was not in the saliva itself-as, indeed, has been suggested.

\section{Conclusions.}

I. It is evident that the saliva is a fluid extremely variable in its composition and anount, but that these variations do not occur without reason, but rather in obedience to fixed and definite laws and in response to certain ascertainable stimuli.

2. The mechanism controlling salivary secretion is extremely

* Various authors quoted by Halliburton in Schäfer's “ Textbook of Physiology," p. I 5 .

$\dagger$ Fleckseder, Centralblatt für innere Medicin, I905, vol. xxvi., p. 4I

† Mosler, Archiv für Heilkunder.

$\S$ American Journal of Physiology, April I, I908. 
sensitive and complex, since different "flavours" of little intensity are capable of being "selected," and give rise to secretions of saliva differing widely in character and amount.

3. That practically all the normal constituents of saliva are, if present in sufficient amount, of value and importance in protecting the teeth against the occurrence of dental caries, and in maintaining the health of the oral mucous membrane.

4. That acids, and particularly the natural organic acids, are the stimulants which excite the greatest amount of these protective substances per minute, and, moreover, give rise immediately, and for a considerable time afterwards, to an increased alkalinity of the mouth. That, conversely, substances of little or no distinctive flavour, and also alkalies, produce a diminution in the amount of protective substances per minute, and reduce the alkalinity of the mouth both at once and for some time afterwards.

5. That in the saliva is provided a natural and potentially perfect mouth-wash acting continuously day and night (not merely for a few minutes a day). That it is, moreover, completely under control; that it may be altered or varied in amount or composition; that its beneficial effects may be increased or decreased absoluetly at will. 


\section{THYROID INSUFFICIENCY AND DENTAL CARIES}

OF recent years attention has been drawn by Leonard Williams* and others to the fact that calcium utilization in the body is intimately associated with the metabolism of the thyroid gland. It is supposed that the internal secretion of the thyroid acts as a lime-salt fixative in the body, and that, when it is absent or deficient, formative organs are unable to utilize or to hold the salts present in the blood, and so the bones and teeth do not calcify to the normal extent.

From clinical evidence I am inclined to think that there is an association between that condition seen in children which is now diagnosed as "thyroid insufficiency" and the presence of dental caries. This, however, is an exceedingly difficult matter to decide definitely: there are so many concomitant circumstances relating to habits and to food which require to be considered and eliminated.

Some writers on the subject would go obviously too far in attributing health or disease of the teeth entirely to the effect of the thyroid gland. H. E. Waller, $\uparrow$ for instance, claims that by giving thyroid extract to a child who had very carious deciduous teeth the condition of the first permanent molar and central incisor teeth was affected, and that, when they erupted, they appeared " to be all that could be desired."

Such an argument is, of course, by itself very fallacious, but in addition it is necessary to point out that at the age

* Medical Review, May, 1910; “Thyroid 'Therapy,” by H. E. Waller, I9I3. $\dagger$ Op. cit., p. 4. 
when the thyroid extract was given (six years) the crowns of the first permanent molars and central incisors were already completely formed. This case would rather go to show that in spite of thyroid insufficiency teeth can be well formed.

\section{Effect of Thyroidectomy.}

In order to obtain some precise data as to the possible effect on the teeth produced by loss of the internal secretion of the thyroid, thyroidectomy has been performed on a number of young rabbits. The result of this operation in rodents is admittedly various, some authorities stating that the animals survive, others that the effect is rapidly fatal. My experience supports this. I succeeded in getting only one rabbit to live; the others (four) died within a week. The animal which survived was kept for twelve months, and then killed. It had thrived well, and was apparently perfectly healthy. Post mortem there was no trace of thyroid gland present.

\section{Alimentary Absorption.}

In order to ascertain to what extent any deficiency of development might be associated with deficient digestion or absorption in the intestines, the amounts of undigested starch and of calcium in the frces were estimated on several occasions, and compared with that of normal animals.

I found that the amount of calcium in the fæces of a thyroidectomized rabbit was in excess of that in the controls, but that starch was much better digested than normally.

The average figures are as follows :

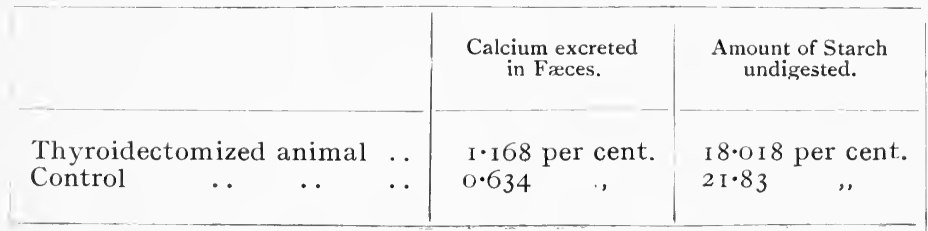


The amount of starch undigested in the fæces is decidedly low; I have not previously observed it in normal rabbits to be below 20 per cent. under any circumstances. This increased utilization of starch probably accounts for the animal's generally fat condition.

The loss of calcium is considerably in excess of the normal, and it is conceivable that this might have a prejudicial effect upon the calcium content of the bones and teeth.

\section{Composition of the Teeth after Thyroidectomy.}

The teeth were to the naked eye well formed and normal, the only difference being that they were extremely white and quite devoid of that yellow staining and black deposit which is almost universally present on rodents' and other animals' teeth (and also in many cases on human teeth immune to caries).

The specific gravity of the teeth was estimated by the pycnometer method, and compared with that of similar teeth in controls. I find it is necessary to use similar teeth, since there is quite an appreciable difference between the incisor and the molar teeth of the same animals, due most probably to the different proportionate amounts of enamel present in the two kinds of teeth. In this case the molar teeth were used. The control teeth gave a specific gravity of $2 \cdot 49$, while those of the thyroidectomized animal gave a slightly lower figure-2:46. This difference is not apparently great, but I have previously shown that the enamel of human teeth demonstrably different in several physical properties differs only to a similarly slight degree in density.

Corresponding with the above, the teeth of the thyroidectomized animal showed on analysis slightly less ash than the controls-namely, 79.I6 per cent. and so per cent. respectively; that is, the teeth after thyroidectomy contained 0.84 per cent. more organic matter than the normal. The 
calcium in the teeth was estimated, and showed that the ash of the thyroidectomized animal's teeth contained 2.I per cent. less calcium than that from the controls' teeth.

\section{Effect on Salivary Secretion.}

It might be thought on a priori grounds that the thyroid and the salivary glands would have something in common. Developmentally they both arise as diverticula from the mouth or oropharynx, and at first the thyroid has also a duct - the thyroglossal-which opens into the mouth (at the foramen cæcum); and both may be associated with the utilization of lime salts. It has also been found that in monkeys, after thyroidectomy, the salivary glands swell up and contain an excess of mucin, but whether this effect is temporary or permanent I am unable to say.

The saliva was, therefore, on seven different occasions obtained from the thyroidectomized and the control rabbits by aspiration from the mouth for five minutes after the subcutaneous injection of pilocarpine (I milligramme per kilo).

The following are the results :

\begin{tabular}{|c|c|c|c|}
\hline & & $\begin{array}{l}\text { Average Alkalinity of } \\
\text { Saliva per C.C. }\end{array}$ & Alkalinity Index. \\
\hline Thyroidectomized rabbit & . & 0.65 & $0 \cdot 336$ \\
\hline Controls $\quad \ldots \quad \ldots$ & . & 0.85 & $0 \cdot 399$ \\
\hline
\end{tabular}

It is seen, therefore, that apparently thyroidectomy has somewhat reduced the output of alkaline salts from the salivary glands, and also that this is not made up for by any increase in quantity of saliva, as shown by the diminution in the index. The amount of saliva obtainable per minute, however, was more variable in the thyroidectomized rabbit than in the controls, and was frequently slightly in excess of 
theirs; but it cannot be claimed that the effect is very considerable either on amount or on alkalinity.

Of more interest, however, is the calcium secretion of the salivary glands, since it is the metabolism of calcium that the thyroid presumably more directly affects.

Saliva was obtained in a similar manner to the above,* and estimated volumetrically for calcium. I found that the saliva of the thyroidectomized rabbit contained slightly less calcium than the control-namely:

\begin{tabular}{c|c|c|c|} 
& Control. & $\begin{array}{c}\text { After } \\
\text { Thyroidectomy. }\end{array}$ \\
\hline Calcium percentage in saliva & $\ldots$ & $0 \cdot 0220$ & $0 \cdot 0206$ \\
\hline
\end{tabular}

That the salivary glands may tend to show a correlated variation with the thyroid gland is supported by the fact that post mortem the submaxillary salivary glands were found to be much below the normal in proportion to the weight of the animal. Thus, the average weight of submaxillary salivary glands in normal full-grown rabbits I have found to be 0.3473 gramme per kilo of body-weight, whereas in the thyroidectomized rabbit it was only 0.226S per kilo.

The above results may be tabulated as on p. 237.

\section{Conclusion.}

It is doubtful whether a definite conclusion can be drawn from the above investigation, seeing that only one animal was used. Further, the animal of course showed no signs of myxœdema. It is nevertheless to be observed that the variations in the thyroidectomized animal, although slight, are all in one direction-namely, in that which would lower

* Two milligrammes of pilocarpine per kilo of body-weight were injected; a period of fifteen minutes was allowed to elapse, and then the saliva was collected by aspiration for about half an hour. 
the resistance of the teeth to disease. This coincides with clinical observations. The result, therefore, goes to support the theory that thyroid insufficiency may slightly favour the occurrence of caries of the teeth.

\begin{tabular}{|c|c|c|c|c|c|}
\hline & & & & $\begin{array}{l}\text { Control } \\
\text { Animals. }\end{array}$ & $\begin{array}{c}\text { Thyroidec- } \\
\text { tomized } \\
\text { Animal. }\end{array}$ \\
\hline $\begin{array}{l}\text { Specific gravity of teeth } \\
\text { Composition of teeth : }\end{array}$ & $\cdots$ & $\cdots$ & $\cdots$ & $\begin{array}{r}\text { Per Cent. } \\
2 \cdot 4900\end{array}$ & $\begin{array}{r}\text { Per Cent. } \\
2 \cdot 4600\end{array}$ \\
\hline Mineral matter .. & . & . & . & $80 \cdot 0000$ & $79 \cdot 1600$ \\
\hline Organic matter .. & . & . & . & $20 \cdot 0000$ & $20 \cdot 8400$ \\
\hline Calcium in ash ... & . & . & . & $40 \cdot 0000$ & $37 \cdot 2100$ \\
\hline $\begin{array}{l}\text { Calcium in dried teeth } \\
\text { Excretion by faces: }\end{array}$ & $\cdots$ & . & . & $32 \cdot 0000$ & $29 \cdot 9000$ \\
\hline Calcium $\ldots$ & . & $\ldots$ & $\ldots$ & 0.6340 & $\mathrm{I} \cdot \mathrm{I} 68 \mathrm{O}$ \\
\hline Starch $\quad \ldots$ & . & . & .. & $21 \cdot 8300$ & I $8 \cdot 0$ I $8 \mathrm{C}$ \\
\hline Salivary secretion : & & & & & \\
\hline Alkalininty per c.c. & . . & . & $\ldots$ & $0 \cdot 8500$ & $0 \cdot 6500$ \\
\hline Alkalinity per minute* & $\cdots$ & $\because$ & . & $0 \cdot 3990$ & $0 \cdot 3360$ \\
\hline Calcium $\quad \ldots \quad \ldots$ & .. & . & . & $0 \cdot 0220$ & $0 \cdot 0200$ \\
\hline Weight of salivary glands & per & of & dy- & & \\
\hline weight $\quad \ldots \quad \ldots$ & . & . & .. & $0 \cdot 3473$ & $0 \cdot 226 S$ \\
\hline
\end{tabular}

* In terms of $\frac{\mathrm{N}}{50} \mathrm{NaOH}$. 
THE OXYGENETIC FACTORS, AND THE MEANS OF THEIR REDUCTION 



\section{THE REDUCTION OF THE CARBOHYDRATE FACTOR BY PHYSIOLOGICAL MEANS}

Iт has previously been pointed out that the presence of both carbohydrates and organisms in the mouth is necessary for the formation of the acid which decalcifies the enamel. It is obvious, too, that if it were possible to eliminate either of these two factors absolutely, caries would ipso facto cease to occur. As a matter of fact, it is extremely doubtful whether this is possible, so that it is necessary to confine our attention to the means of reducing both factors to such an extent that their combined action will be less than the resistance, both passive and active, offered by the oral tissues and secretions. We consider here the reduction of the carbohydrate factor.

It is obviously necessary first of all that we should know the relative fermentability of the various carbohydrates in daily use, in order that we may know which to avoid and which to advocate. With the object of gaining precise data on this point, the following series of experiments have been carried out. Throughout these experiments the amount of fermentation in a given time is expressed by the number of c.c. of $\frac{\mathrm{N}}{5} \mathrm{NaOH}$ required to neutralize the acid formed. Since the results are needed for comparison only and not for absolute measurement, this method is accurate enough and has the advantage of simplicity, and the comparative variations in the amount of acid can be seen at a glance.

The indicator used was neutral litmus solution. This has 
the disadvantage of being affected by carbon dioxide, but other indicators which are unaffected by this agent are unsuitable for organic acids.

The possible carbon-dioxide error cannot be removed by boiling the liquid, since the acid which is almost invariably formed by mouth organisms fermenting carbohydrates is the inactive ethylidene lactic acid, or $a$-hydroxypropionic acid-

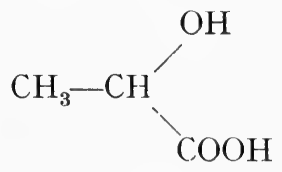

-and this loses its acid properties upon being heated.

Except where otherwise stated, the subject of the experiments was always the same.

\section{Lactic Fermentation in Vitro.}

I. Two grammes of each of the following substances were taken, mixed by trituration in a pestle and mortar with Io c.c. of distilled water, sterilized, infected with $0 \cdot 2$ c.c. of mixed saliva, and incubated for four days. At the end of this time the acid was estimated, with the results as shown in the table on p. 243, which are the averages of three series of estimations.

Such an experiment is obviously wholly in favour of the organisms, and it gives the acid-production when all the substances are under equally favourable conditions, which of course would not obtain in the mouth. Some would be acted on much more than others by ptyalin in the first place before fermentation by the organisms cccurred, some adhere much more than others to the teeth, and some are salivary stimulants; and thus the acid formed would tend to be neutralized more in some cases than others. It represents, perhaps, the comparative amounts of acid which would occur in a very " dry " mouth, if all the substances tended to lodge equally. 
It is seen that all the carbohydrates form some acid, but that there are great differences in the amount formed. For instance, from chocolate is formed more than twenty times as much acid as from cane-sugar, and from parsnips is formed twice as much as from apple.

As is seen from the second column, these differences do not in the least correspond with the amount of carbohydrate

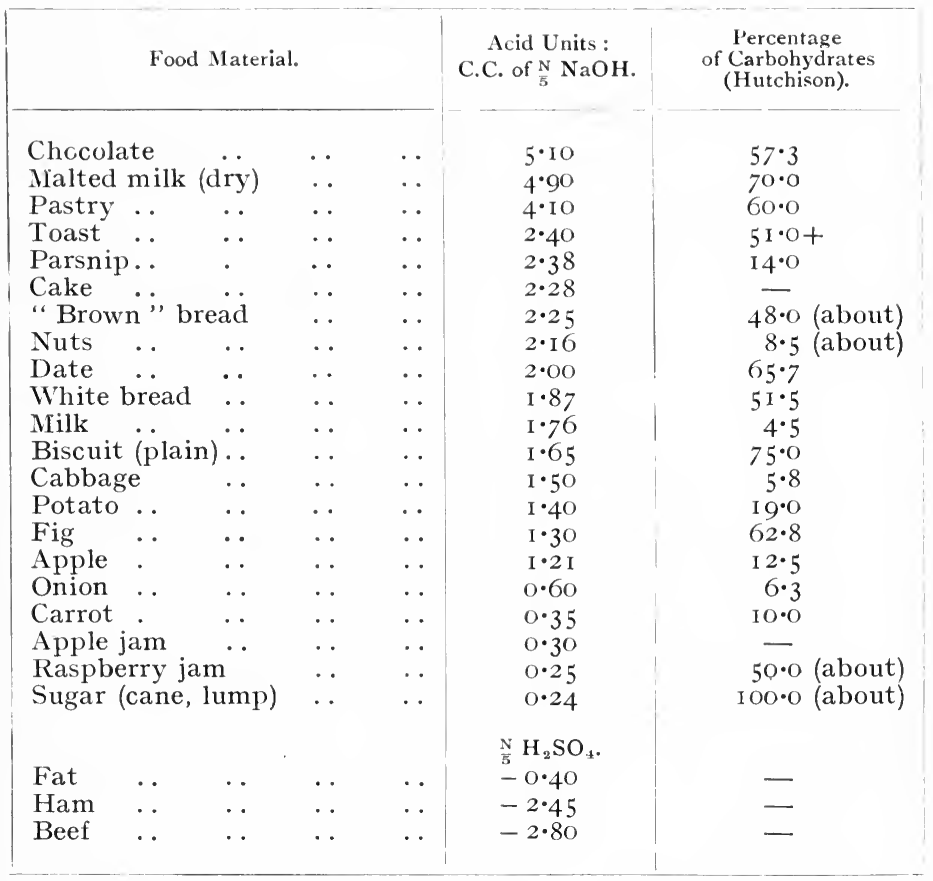

present in each; but rather it depends upon the kind of carbohydrate and its state of comminution. It is, too, a very significant fact that all those substances from which least acid is produced are the most sapid substances, and the majority of them (cabbage, potato, apple, onion, carrot, apple and raspberry jam) are originally acid in reaction. 
The only materials which ga ve an alkaline reaction were the proteins-the beef, ham, and fat.

As regards the fermentability of the various sugars, Aitchison Robertson* places them in the following order for lactic fermentation: (I) Lævulose; (2) lactose; (3) dextrose; (4) canesugar; (5) maltose. Using 2 per cent. solutions of the pure sugars infected with 0.2 c.c. saliva, I obtained the following order: (I) Dextrose; (2) lævulose; (3) maltose; (4) lactose; (5) cane-sugar. Both coincide in placing cane-sugar very low, and certainly the position of maltose in Aitchison Robertson's scale does not hold for the mouth; for, as we shall have occasion to see, and, in fact, have seen for Experiment I., maltose (derived from starch) forms more acid than almost any other kind of sugar. In both lævulose is placed high; but, again, this does not represent practically the amount of acid formed from it in the mouth, since those substances which contain lævulose (except honey) are usually combined with salivary stimulants in such a manner as to negative the acid-production.

\section{The Effect of Insalivation.}

2. Since certain of the substances tested above are salivary stimulants and others are not, and some require much more mastication and are therefore more insalivated than others, and since some are more affected than others by ptyalin, it becomes necessary to know what is the combined effect of insalivation, mastication, and fermentation, on each of the substances. This was estimated under the following conditions: The teeth were cleaned, and one hour afterwards 2 grammes of the substances to be tested were masticated and insalivated, until in each case the desire to swallow the mass was felt. It was then ejected into a sterile test-tube and incubated for four days; at the end of which time the acidity was estimated as before, with the following results.

* Hutchiscn's “Food and Dietetics," p. 277. 
None of the substances were sterilized, and each was used in the form in which it is usually eaten.

Food Material.

\begin{tabular}{|c|c|c|}
\hline Pastry & & . \\
\hline White bre & & .. \\
\hline $\begin{array}{l}\text { Toast } \\
\text { "Brown" }\end{array}$ & $\ddot{\text { bread }}$ & \\
\hline Chocolate & . & .. \\
\hline Biscuit & .. & .. \\
\hline Apple & & \\
\hline Potato & & \\
\hline Bread and & 1 butte & r, with \\
\hline Crust of $b$ & read & .. \\
\hline Parsnip & . & 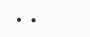 \\
\hline Orange & .. & .. \\
\hline Salad & & 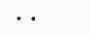 \\
\hline Cane-suga & & . \\
\hline Rice & & .. \\
\hline Neat & & . . \\
\hline
\end{tabular}

Acid Units in Four Days :

C.C. of ${ }_{5}^{\mathrm{N}} \mathrm{NaOH}$.

The first thing which strikes one in comparing this latter list with the former is that several of the substances have changed their positions. This, of course, is due (I) to the neutralizing effect of the increased amount of saliva promoted by some of the substances, and (2) to the presence of an increased amount of ptyalin, thus converting more starch into maltose. Those substances which have taken a higher place in the list are those on which the increased ptyalin has acted, giving rise more rapidly to a supply of maltose, which the organisms have converted into lactic acid. Those substances which are lower are so because they have induced an extra flow of alkaline saliva, which has been sufficient to more than neutralize the increased effect of the ptyalin. This may be taken to represent the amount of lactic fermentation which would take place in any ordinary mouth, provided equal quantities of all food substances remained after mastication to be fermented. That is to say, supposing spaces existed between the teeth in a certain mouth and that they were always filled up after each meal, irrespective of what composed the meal, then the above table might be taken to 
represent proportionately the amount of acid which would be formed from each of the substances, and it represents for enamels of equal resistance the amount of destruction of the surface which would take place. It is perfectly clear that the material having the greatest potentiality for tooth destruction is starch in a cooked and finely divided state. Although, of course, all starch must first be converted into maltose before lactic fermentation can take place, yet it is important to notice that much more acid is formed from starch than from an equal quantity by weight of maltose in a given time. Thus, equal quantities of bread and maltose were sterilized and mixed with equal quantities of saliva, and incubated for two days. In an average of three tests the bread gave 0.36 acid unit, and the maltose o.I6. This is probably due, as Ellenberger and Hoffmeister* have suggested, to the more ready fermentability of maltose in a "nascent" condition.

Here, then, is amply borne out by experimental proof the fact that precisely those articles which form the greater bulk of the diet of the higher civilized peoples at the present time are those which have the greatest potentiality for acid-production. Particularly is to be noticed the large amount of acid formed by pastry, bread ("brown" and white), toast, chocolate, and biscuits. Another point of importance is the relatively small amount of acid produced from cane-sugar and from rice. The amount of acid formed from cane-sugar is more than in the former table owing to the larger number of organisms present, but its position is relatively much lower owing to the increased amount of saliva induced reflexly.

That so little acid should be produced from rice (boiled) is a little surprising at first, until it is remembered what a relatively small surface is exposed to the action of the ptyalin and organisms (i.e., the starch is not in a finely divided,

* Archiv f. Wissensch. u. Prakt. Thierheilk., Bd. xi., S. I62. 
" refined " state). Again it is noticeable that the most sapid substances are responsible for the least acid ( $c f$. apple, potato, parsnip, orange, salad, and cane-sugar). Bread crust, also, by reason of the increased mastication, and therefore salivation, has only developed about one-half the acid that the soft white bread has.

Protein material (meat) under these circumstances shows a strong alkaline reaction-more alkaline, in fact, than the bread was acid; it might be thought, therefore, that the two would neutralize each other; but, as we shall show, this is not so. Ideally, of course, similar experiments should be carried out with a person immune to caries; this, unfortunately, has not been possible, but one substance only-white bread-has been tested in the same way with two immune persons. The first gave an acidity of $\mathrm{I} \cdot \mathrm{O}$, and the second $2 \cdot 4$, both considerably less than the figures above. Both of these low acidities are due, I think, to the increased neutralization of the acid formed, since in both (and especially the first) the fluidity of the expectorated mass was marked.

Fermentation was not complete in any of the substances at the end of the four days, since, upon testing, they all reduced Fehling, hence showing that sugar was still present. Control fermentations tested with Uffelmann's reagent showed lactic acid to be always present. Occasionally the presence of acetic acid was shown by a brown reaction, or $\beta$-hydroxypropionic acid by the acidity to litmus remaining after boiling.

That in a mixture of protein and carbohydrate the alkaline decomposition of the one does not neutralize the acid fermentation of the other is shown by this experiment: Eight mixtures, each of bread I gramme and meat I gramme, were insalivated and placed in sterile tubes; seven were incubated, and a different tube was examined each day for a week, with results as follows : 


\begin{tabular}{|c|c|c|}
\hline $\begin{array}{c}\text { Number of } \\
\text { Tube. }\end{array}$ & $\begin{array}{c}\text { Number of Days } \\
\text { iucubated. }\end{array}$ & $\begin{array}{c}\text { Acid Units developed : } \\
\text { C.C. } \frac{\mathrm{N}}{5} \mathrm{NaOH} .\end{array}$ \\
\cline { 1 - 2 } $\mathrm{I}$ & after standing one hour & neutral \\
2 & $\mathrm{I}$ & $2 \cdot \mathrm{I}$ \\
3 & 2 & $3 \cdot 7$ \\
4 & 3 & $4 \cdot 6$ \\
5 & 4 & $5 \cdot 8$ \\
6 & 5 & $5 \cdot 6$ \\
7 & 6 & $5 \cdot 9$ \\
8 & 7 & $6 \cdot \mathrm{I}$ \\
\hline
\end{tabular}

The acid-production obviously increases rapidly up to the fourth day; after that it still increases, but more slowly. Any neutralization which might occur after the seventh day would be quite valueless, since by that time, as will be shown next, a considerable portion of the enamel would have been decalcified. It is seen that on the fourth day more acid has been formed from the bread and meat mixture than from the bread alone (see table on p. 245). This fact has been repeatedly noticed-that the admixture of protein seems to increase the rapidity of the acid-formation in carbohydrates. Therefore not only is such a mixture not to be regarded as beneficial, but as positively harmful.

Note.-After more extended observations on this point, I am inclined to the opinion that the large amount of protein débris mixed with fermentable carbohydrates constitutes one of the most important factors in the present prevalence of dental caries. The presence of abundance of protein seems to stimulate either proliferation or general vigour of the acidforming organisms. (See Chapter XVIII.)

\section{Decalcification of Enamel.}

3. That the order in which the various food materials have been placed thus far corresponds to their conıarative destructiveness to enamel is supported by the following experiment: 
Two grammes of bread, apple, and cane-sugar, were each triturated with Io c.c. of water, sterilized, and infected with 0.2 c.c. of mixed saliva. The crown of a perfectly sound lower molar tooth (malacotic in type) was divided into three equal portions, and a portion placed in each of the mixtures and incubated for one week.

The following table expresses the result:

\begin{tabular}{|c|c|c|c|c|}
\hline \multicolumn{3}{|c|}{ Material. } & $\begin{array}{l}\text { Acid Units: } \\
\text { C.C. } \mathrm{N} N \mathrm{NaOH} \text {. }\end{array}$ & Condition of Enamel. \\
\hline Bread .. & . & . & $2 \cdot 5^{*}$ & Enamel opaque, white all \\
\hline Apple . . & . & . & $0 \cdot 6$ & Slightly opaque at edges and \\
\hline Sugar (cane) & . & $\ldots$ & $0 \cdot 4$ & $\begin{array}{l}\text { Very slight opacities near } \\
\text { edges and cusps. }\end{array}$ \\
\hline
\end{tabular}

The accompanying illustration (Fig. $7 \mathrm{o}$ ) is a photograph of the three portions of crown after removal from the mixtures. The differences in opacity can be readily seen. On each portion a cross was scratched with a sharp needle; the effect is only visible on the portion from the bread mixture, and the depth is about 0.5 millimetre (or about one-fourth of the total thickness of the enamel).

\section{Lodgeability of Foodstuffs.}

4. In the foregoing experiments there is one most important factor which has so far been entirely neglected-the relative tendency of the various materials to lodge on or between the teeth. It is a well-known fact that certain articles of diet tend to stick more firmly to the teeth, or to leave more débris behind after mastication, than others. It is perfectly obvious, therefore, that in a normally shaped mouth, if a substance had a high acid-potential but yet did not lodge, it would

* Lower than in the last table because much of the lactic acid has combined with the lime salts of the enamel. 
not be anything like so harmful as another substance which had a low acid-potential, but a great tendency to lodge on or between the teeth. The relative "lodgeability" of foodstuffs is therefore a matter of the highest importance, and one on which it is necessary to have some precise information. After trying several other plans for determining this, the following method was adopted:

The teeth were first carefully cleaned with a tooth-brush (the form of which will be described later) and water. One

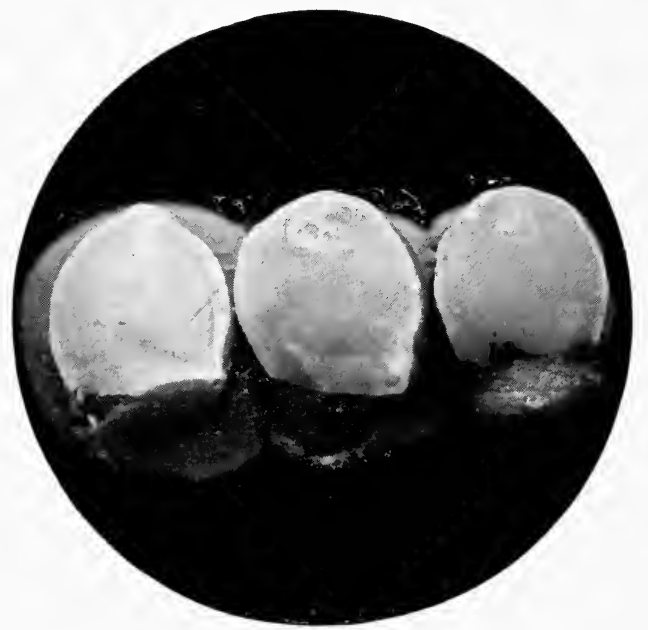

Fig. 70.-Three Portions of the Crown of a Tooth after being SUbjected for One Week to the Fermentation of Bread, Apple, and Sugar (from Left to Right respectively).

hour afterwards the substance to be tested was eaten for about three minutes in as natural a manner as possible. After the lapse of five minutes the teeth were again cleaned with Io c.c. of distilled water, and the débris carefully collected from the brush in a special-shaped beaker with an inverted rim. (In order to avoid undue external infection, the brush and beaker were previously washed for some minutes with alcohol, and the latter allowed to evaporate.) 
The actual time of brushing was in each case one minute, and care was taken to prevent saliva from entering the beaker. The mouth was afterwards rinsed out with another Io c.c. of distilled water, and the whole 20 c.c. then incubated in a sterile flask. The period of incubation chosen was twenty-four hours, since after this time some of the substances which had developed acid were found to become alkaline owing to the carbohydrate being all used up, and to the organisms turning their attention to the protein constituents of the saliva.

The tables on p. 252 give the average results of several acid estimations obtained in such a manner.

These figures may be taken, then, as representing the resultant of all the various forces-acid-forming, adhesive, neutralizing, and cleansing-at work in the mouth. It is the only test which determines practically for an "average" mouth the relative harmfulness or otherwise of the various articles of diet; we may therefore say definitely that the order in which the materials are placed here represents their relative potential for initiating caries of the teeth, with the exceptions that lemon is probably more beneficial and cane-sugar more harmful than these tables show.

All the materials in the first table have a potential for causing caries; all those in the second table have either no such tendency or one which is very readily neutralized. The lodgeability of the various materials evidently does not depend upon the amount of fibrous or inert matter they contain. As is shown by the second column, the amount of acid or alkali formation bears no proportion to the amount of cellulose contained in the material. Compare, for instance, orange and brown bread; both contain the same amount of cellulose, yet the former gives an alkaline reaction of 0.5 , and the latter an acid reaction of 0.4 ; and figs, with 7.3 per cent. of cellulose, result in an acid reaction, whilst bananas, with only 0.2 per cent., have an alkaline result. Several substances, too, with 
no cellulose at all yield less acid than those with a moderate amount. It is evident, therefore, that some other explanation must be sought to account for the variations.

There is one property which sharply differentiates these two series of foodstufis. It is their acidity or alkalinity.

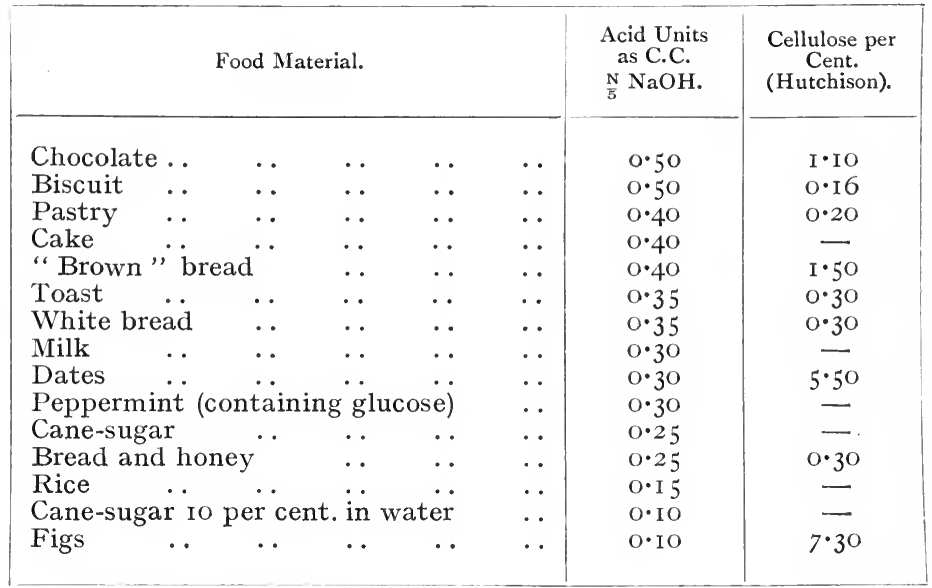

\begin{tabular}{|c|c|c|c|c|c|c|}
\hline \multicolumn{5}{|c|}{ Food Material. } & $\begin{array}{l}\text { Alkalinity as C.C. } \\
\mathrm{N}_{\mathrm{H}_{2} \mathrm{SO}_{4}}\end{array}$ & $\begin{array}{l}\text { Cellulose per } \\
\text { Cent. }\end{array}$ \\
\hline Potato .. & .. & .. & .. & $\ldots$ & $-0 \cdot 10$ & $0 \cdot 60$ \\
\hline Lemon .. & $\ldots$ & $\ldots$ & . & $\ldots$ & $-0 \cdot 15$ & - \\
\hline Parsnip.. & $\ldots$ & .. & $\ldots$ & . & $-0 \cdot 20$ & $0 \cdot 72$ \\
\hline Pineapple & .. & .. & .. & .. & $-0 \cdot 22$ & - \\
\hline Banana.. & .. & .. & $\therefore$ & $\therefore$ & -0.25 & $0 \cdot 20$ \\
\hline Apple .. & .. & .. & . & $\therefore$ & $-0 \cdot 30$ & $2 \cdot 70$ \\
\hline Nuts .. & $\ldots$ & .. & $\ldots$ & $\ldots$ & -0.50 & $5 \cdot 20$ \\
\hline Orange... & $\ldots$ & .. & $\ldots$ & $\ldots$ & -0.50 & $I \cdot 50$ \\
\hline Meat (beef) & $\ldots$ & .. & $\therefore$ & $\therefore$ & $-0 \cdot 50$ & - \\
\hline Fish .. & .. & .. & . & .. & -0.87 & 一 \\
\hline Duck .. & . & .. & . & $\ldots$ & $-\mathrm{I} \cdot 80$ & - \\
\hline
\end{tabular}

All the substances which are in the first table, and therefore harmful, are originally either alkaline or neutral in reaction; all the carbohydrate substances (with the exception of nuts) which are in the second table, and therefore beneficial, 
are originally acid in reaction. In other words and in general terms, salivary depressants tend to lodge and remain in the mouth more than salivary excitants.

Two series of the same substances similarly treated were also incubated for four and eight days, in order to determine how long it took the organisms to use up the carbohydrate in the several instances. At the end of four days the substances which showed acid reaction were-

$\begin{array}{ll}\text { Pastry. } & \text { Biscuit. } \\ \text { Toast. } & \text { Crust of bread. } \\ \text { Chocolate. } & \text { Cane-sugar. }\end{array}$

At the end of eight days the only substance which remained acid was cane-sugar; white bread was neutral, all the remainder being strongly alkaline.

Here again it is seen that six of the most common articles of our modern dietary are the ones which give rise to the most prolonged acid reaction in the mouth.

The position of cane-sugar is hereby completely reversed, and from an apparently harmless form of carbohydrate it becomes (in this form, at least) equal to the most harmful ones, owing to its prolonged action. The reason for this difference of behaviour of cane-sugar in vitro and in the mouth is to be found in the fact that this substance is, as has been shown, a powerful excitant of the mucous glands of the mouth. The resulting viscous mucin binds the sugar to the teeth, and, not being acted upon by ptyalin, it remains and is slowly fermented by the organisms alone.

\section{REDUCTION OF THE CARBOHYDRATE FACTOR.}

The question which now arises is, Can we by a combination or sequence of salivary depressants and excitants reduce the fermentable débris remaining in the mouth* after a meal ?

* "In the mouth," and not in apposition to the teeth alone; since the débris which remains in the buccal sulcus, for instance, forms lactic acid, and this, combining with alkaline salts of the saliva, con- 
To consider first of all a sequence. Substances which were known to produce most acid were eaten, and then followed immediately by other substances which had been shown to result in an alkaline reaction. The teeth were then brushed and the mouth rinsed, and the washings incubated as before, with the following results:

\section{Substance.}

Bread and butter followed by apple .. Bread and butter followed by orange. . Bread followed by radish Bread followed by fish .. Bread followed by duck Rice followed by orange Chocolate followed by apple Cake followed by orange Bread and butter and caramels followed by kaurigum (chewed)*

Here, then, is an important fact, that by such a sequence the deleterious effects of acid-forming substances can be completely overcome, and an acid result transformed into a neutral or alkaline one. This, obviously, is of the utmost importance as a physiological means for the prevention of caries, and offers a most siniple and efficacious method whereby the commencement of the process of decay may be obviated.

The method would probably not be equally efficacious if carious cavities were already present and unfilled; it could not, however, be other than beneficial; but this aspect does not immediately concern us. Given a mouth in which the teeth are fairly regular and in which there are no gross cavities to retain the previously-eaten soft fermentable food, then we may with safety say that, if such food be followed by articles of alkaline potential and salivary excitants, the tendency

siderably reduces its power of neutralizing the acid formed near the teeth; particularly does this apply to parotid saliva and its already somewhat feeble protective properties.

* This substance was chewed extensively by the Maoris, and was probably a factor in producing their remarkable freedom from caries. 
to caries will be enormously reduced or entirely negatived. I see no reason whatever why such a sequence should not always, and at every meal, be practicable; but in case it should not be so, we consider the next question:

Can we by the use of a combination of such substances attain the same results?

The following table, being the results of estimations made in a similar manner to the above, but the substances being mixed and not in sequence, supplies an answer:

Substance.

Acid or Alkaline Units :

$\frac{\mathrm{N}}{5} \mathrm{NaOH}$ and $\mathrm{H}_{2} \mathrm{SO}_{4}$

$\begin{array}{llll}\text { Bread and butter with raspberry jam .. } \quad \ldots \quad \ldots & \ldots & \text { in } 24 \text { Hours. } & 0.5 \text { acid }\end{array}$

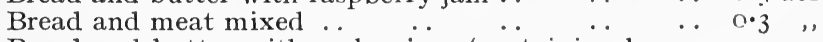

Bread and butter with melon jam (containing lemon

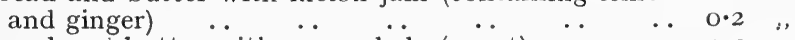

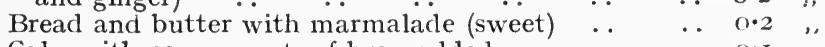

Cake with 30 per cent. of bran added.. $\quad \ldots . \quad \ldots 0^{\circ}$ " ",

Bread and butter with quinine, I in $800 \ldots 0.3 \quad \ldots$.

Bread and butter with acid potassium tartrate $\quad . \quad$ Neutral

Bread and butter with acid potassium tartrate and

o•OI per cent. saccharine

Biscuit with tartaric acid I per cent.

Bread and butter with tartaric acif i per cent. .. 0.08 "'alkaline)

Cane-sugar with tartaric acid I per cent. . . . . $0.05 \quad$,.

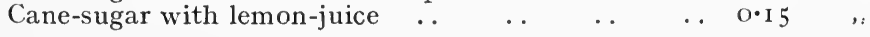

In this case the results are not as marked. It is obvious that a more powerful salivary stimulant must be mixed with the bread in order to reduce its lodgeability, and therefore its acid-production.

It will be noticed that raspberry jam had the effect of increasing the acid production, whilst " melon " jam slightly reduced it. Quinine had practically no effect at all. Only tartaric acid and its potassium salt were sufficient to prevent acid-production.

The fact of rendering a substance very fibrous by the addition of bran, although reducing the acid-production somewhat, does not prevent it, and is nothing like so efficacious as the acids. The mixture of meat with bread likewise has very little effect, only reducing the acidity from 0.35 to $0 \cdot 3$, 
but since the meat probably exercised a certain detergent effect, this amount of acid has been formed from a very much smaller quantity of bread. This was quite obvious from a naked-eye examination of the washings. With regard to "sugar," we now observe what is a most important principle -i.e., that by its combination with an acid not only are its lodgeability and acid potential negatived, but for the first time an alkaline result is obtained. This is to be accounted for by the fact that the acid precipitates the mucin, and therefore it no longer has the power to bind the sugar to the teeth; the resulting extra flow of a thin alkaline saliva washes the sugar clear of the teeth, and leaves only an alkaline deposit, or one which, on decomposition, yields alkaline substances.

A similar explanation accounts, of course, for the alkaline result from the bread, but in this case the mucin factor is not present-at least, to anything like the same extent.

It has been insisted upon always by physiologists that sugar is a valuable food, and therefore an article of diet to be recommended; but dental practitioners have always equally insisted that the consumption of sugar is a potent factor in the production of caries, and one, therefore, to be avoided. Here, then, we have a means of giving sugar-by combining it with an acid-which will not only not be harmful to the teeth, but actually beneficial, since the combination of sugar and acids promotes a profuse after-flow of alkaline saliva.

We have previously seen that the fact of diluting the sugar largely with water (9o per cent.) does not prevent acid-production. There is, therefore, only this means whereby sugar may be prescribed harmlessly.

It is to be noted that it is in this combination that sugar is most commonly provided by Nature-i.e., combined with the acids or acid salts in roots, plants, or fruit. It is therefore a condition which should always be enjoined when advising the consumption of sugar as a food, especially in regard to children. 


\section{Sugar-Cane.}

The example of native children eating large quantities of sugar-cane is frequently quoted as evidence that cane-sugar cannot be productive of dental caries, since such children have for the most part quite healthy teeth. This is a typical example of the kind of argument which is too frequently seen - a perfectly correct premiss, but a quite incorrect inference.

I have obtained a sample of fręsh sugar-cane from Fiji in a hermetically sealed case, and have tested it with a view to ascertaining its possible effect on the teeth. The tests applied were those which have previously been used in connection with other foodstuffs, and the results of which have just been considered.

The following are the observations as regards sugar-cane:

r. The juice of the sugar-cane is markedly acid.

2. The saliva induced reflexly from the mastication of sugarcane was collected by means of the cannula and segregator from one side of the mouth. It amounted to 3.5 c.c. per minute, or 7 c.c. for the whole of the glands.

The alkalinity of the saliva was 0.94 units (c.c. $\frac{\mathrm{N}}{50} \mathrm{H}_{2} \mathrm{SO}_{4}$ ) per c.c., or an alkalinity index of 6.58. (See Table I., p. r6r.)

This is more than five times that evoked by bread and butter.

3. The débris remaining in the mouth and on the teeth was collected and incubated for twenty-four hours, and at the end of that time showed a mean alkaline reaction of $0^{\circ} 3 \mathrm{I}$ units $\left(\frac{\mathrm{N}}{5} \mathrm{H}_{2} \mathrm{SO}_{4}\right)$, thus placing it between apple $(0 \cdot 3)$ and orange (o 5) from a protective point of view. (See Table, p. 252.)

4. The sugar-cane is, of course, very fibrous, and it has been suggested that immunity to caries may be due to this fact. That may be so to a certain extent, but it is more likely due to the acid reaction of the juice. For instance: (a) Sugar-cane was masticated thoroughly, including the 
fibres, and the débris collected and incubated. The alkalinity developed was equal to 0.26 units. (b) Sugar-cane was imperfectly masticated, but the juice thoroughly sucked out, and the reaction of the incubated débris again estimated, when it amounted to 0.37 alkalinity units; whereas a ro per cent. solution of refined cane-sugar-i.e., minus its acidgave rise to an acid-formation of $O \cdot I$ unit.

It would therefore appear that more protection is afforded from the acid juice than from the fibrous elements, which are distinctly "woody" and devoid of flavour, and therefore of any reflex stimulating effect upon the salivary glands.

There is also a vast difference in the concentration of canesugar as taken by natives in sugar-cane and as taken in the form of lump-sugar or sweets. It requires about twenty sticks of sugar-cane to make I pound of lump-sugar. This means that every large "lump" of sugar is equal to 2 feet of cane. European children frequently, therefore, eat the equivalent of at least Io or I2 feet of cane in a few minutes, while many adults consume the equivalent of 2 to 6 feet of cane in a cup of tea. Now, Polynesian natives inform me that they usually take a piece of cane 2 to 3 feet long, and this takes them about half an hour to consume. It is quite obvious that no comparison whatever can be drawn between the consumption of raw sugar-cane and of cane-sugar in the refined and concentrated form in which we know it.

It has been stated that since the introduction of sugar-cane into the Pacific Islands the natives' teeth have deteriorated. Such a view, however, must be based on a misconception, for Captain Cook,* in the account of his I768-7I journey, says: "The product of the island [Tahiti] is sugar-cane, which the inhabitants eat raw." And Ellis, $\uparrow$ in I83I, spoke of the sugarcane " formerly cultivated," but then wild, and also eaten raw.

* Literal transcripition by Captain Wharton, p. 89.

† "Polynesian Researches," second edition, vol. i., p. 64. 


\section{The Relative Value of Whole-Meal and White Bread.}

This is an extremely debatable subject, but since of recent years a considerable amount of attention has been directed to the matter in connection with the relative values of these two varieties of bread in the formation of bones and teeth, it is necessary, perhaps, to state the present views on the subject.

We may consider the question in two parts:

I. As to the provision of sufficient mineral salts for the proper formation of the teeth.

2. The effect of the débris, after mastication, on the teeth.

I. Provision of Minerals.-A grain of wheat consists of bran, germ, and endosperm. The latter forms the bulk of the grain, and is the only part used in making fine wheat-flour. As regards the disposition of the mineral constituents, the bran con-

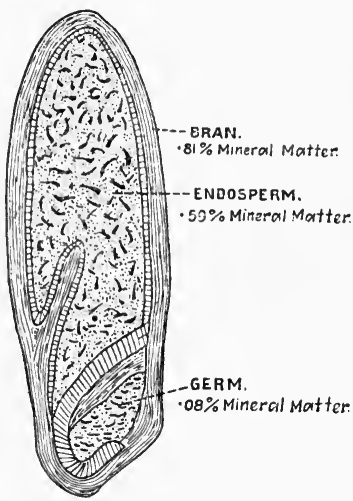

Fig. 7I. - Diagrammatic Section of a Grain OF WHEAT, SHOWING THE Amount of Mineral MatTer in the VARIOL'S PARTS. tains $0.8 \mathrm{I}$ per cent., the germ 0.08 per cent., and the endosperm 0.59 per cent.; thus the endosperm contains a little more than half the mineral matter.

The effect of milling on wheat is shown in Table A, on p. 260.

It will be observed that the proportion of ash (minerals) remains about the same in the two varieties, as was stated above for the various parts of a wheat-grain - that is to say, roller-milling deprives the flour of not quite one-half of its minerals. A stage further, and Table B, on p. 260 , gives the composition of three types of bread.

The proportions are not so divergent, but still marked. 
It is, however, not sufficient to know the composition of a food; what is also necessary is to know how it is digested and absorbed, since, obviously, if mineral matters pass straight

$$
\text { A.-The Effect of Roller-Milling on Wheat.* }
$$

\begin{tabular}{|c|c|c|c|c|c|}
\hline & & & $\begin{array}{l}\text { Wheat as it } \\
\text { enters the } \\
\text { Mill. }\end{array}$ & $\begin{array}{c}\text { Bakers' Patent } \\
\text { Roller- } \\
\text { Process Flour. }\end{array}$ & $\begin{array}{l}\text { Entire-Wheat } \\
\text { Flour. }\end{array}$ \\
\hline Water & $\ldots$ & $\ldots$ & $\begin{array}{c}\text { Per Cent. } \\
9^{\circ} 66\end{array}$ & $\begin{array}{c}\text { Per Cent. } \\
\text { I I } 99\end{array}$ & $\begin{array}{c}\text { Per Cent. } \\
\text { I I } \bullet 4\end{array}$ \\
\hline Protein & .. & . & I $4 \cdot 18$ & I $3 \cdot 3$ & I 3.8 \\
\hline Fat & . & $\ldots$ & $2 \cdot 16$ & $I \cdot 5$ & $\mathrm{I} \cdot 9$ \\
\hline Starch & $\cdots$ & . & $69 \cdot 94$ & $72 \cdot 0$ & $7 \mathrm{I} \cdot \mathrm{O}$ \\
\hline Crude fibre & .. & . & $\mathrm{I} \cdot 7 \mathrm{O}$ & $0 \cdot 7$ & $0 \cdot 9$ \\
\hline Ash & . & . & $I \cdot 9 I$ & $0 \cdot 6$ & $\mathrm{I} \cdot \mathrm{O}$ \\
\hline
\end{tabular}

B.-Analyses of Breads by Atwater.

\begin{tabular}{|c|c|c|c|c|}
\hline & & Water. & Protein. & $\begin{array}{l}\text { Mineral } \\
\text { Matter. }\end{array}$ \\
\hline $\begin{array}{l}\text { White bread } \\
\text { "Brown" bread } \\
\text { Graham bread } \\
\text { entire grain) }\end{array}$ & $\begin{array}{l}\cdots \\
\ddot{\text { contains }} \\
\ldots\end{array}$ & $\begin{array}{c}\text { Per Cent. } \\
35 \cdot 4 \\
40 \cdot 0 \\
\\
32 \cdot 3\end{array}$ & $\begin{array}{l}\text { Per Cent. } \\
9{ }^{\circ} 5 \\
5 \cdot 0 \\
8 \cdot 5\end{array}$ & $\begin{array}{l}\text { Per Cent. } \\
\quad \mathrm{I} \cdot \mathrm{I} \\
\mathrm{I} \cdot 9 \\
\mathrm{I} \cdot 5\end{array}$ \\
\hline
\end{tabular}

through the alimentary canal, they cannot be considered in connection with tooth development. 'The following figures illustrate this:

C.--Average of Analyses showing Amount of Constituents not Digested AND NOT ABsorben.†

\begin{tabular}{|c|c|c|c|c|c|}
\hline & & & & White Bread. & Whole-Meal Bread. \\
\hline Total solids.. & . & . & . & $\begin{array}{c}\text { Per Cent. } \\
4 \frac{1}{2}\end{array}$ & $\begin{array}{l}\text { Per Cent. } \\
\text { I } 4\end{array}$ \\
\hline Proteins $\quad$. & . & .. & .. & 20 & 25 \\
\hline Carbohydrates & $\ldots$ & .. & . & 3 & 6 \\
\hline Ash ... & $\ldots$ & $\ldots$ & . & 25 & $5 \mathrm{I}$ \\
\hline
\end{tabular}

* As published by the United States Department of Agriculture.

† Hutchison: "Food and Principles of Dietetics," p. 213. 
Here, obviously, we have the crux of the whole problem. Roughly, twice as much mineral matter is lost from wholemeal bread as from white bread, and we have seen that it contained originally not quite twice as much. It is therefore quite obvious that slightly more mineral matter is absorbed from white bread than from whole-meal bread.

It might be thought that the above result was due to the increased peristalsis induced by the whole-meal bread.

Rubner* states, from observation, that the bowels were evacuated somewhat more slowly on a diet of "coarse" bread than on a diet of " fine" bread. Whole-meal bread, however, more readily undergoes acid fermentation in the intestines, and this causes a greater outpouring of alkaline secretion, which may give the impression of loose motions.

Romberg and Snyder have shown, on the other hand, that the absorption of whole-meal bread may be considerably increased by grinding the meal until the whole will pass through a fine sieve. Thus, for instance, Romberg-whose results we quote for mineral constituents, in which we are particularly interested-found that, while the percentage of ash in white flour and whole meal was as $0 \cdot 49: 2 \cdot 22$, the percentage unabsorbed for the same substances was only as $58 \cdot 8: 6 \mathrm{r} 9$. The grinding of the bran, however, to the fineness required to give the above figures is an exceedingly difficult matter, and at present the trouble and expense are almost prohibitive.

Let us for the moment, nevertheless, grant that the above is possible and practicable, and that the mineral constituents of bread could be largely increased: it still remains to prove that this is necessary and desirable, or would effect an improvement in the density of the teeth. We have to remember that lime salts and phosphates are excreted in the urine, and that the more of these substances the food contains the more

* Zeitschrift für Biologie, Band xix., p. 45. 
does the urine excrete. Further, seeing that these salts are always being excreted by the kidneys, it suggests that their non-utilization in tooth formation is not due to any deficiency, but rather to an absorptive defect of the formative organs.

Majendie, Stark, and I-eonard Hill* have all shown that malnutrition (and sometimes death) results from an exclusive diet of white bread, whereas animals thrive well on brown bread. This effect is probably due to the absence of substances, in the nature of ferments, which Hill calls "vitamines," and which are destroyed in the modern process of refining white flour. The addition of a small percentage of fresh food, such as fruit or vegetable, to the white bread prevents any such result. There is no evidence, therefore, to show that in the ordinary dietary of a mother or child whole-meal bread is more advantageous to tooth development than ordinary white bread.

2. The Effect of the Débris, after Mastication, on the Teeth. -Does the additional cellulose, the increased fibrousness, of whole-meal bread lead to a lessening of the débris remaining in the mouth after mastication? No, appreciably not. This may be tested by anyone by eating pieces of white and wholemeal bread, and, five minutes after eating each, washing the mouth out thoroughly with about I ounce of water, then examining the washings in two glasses of equal size side by side. My experience has been, both with individuals immune and susceptible to caries, that more débris remains after wholemeal than white bread. A factor contributing to this may be the fact that in whole-meal bread the germ enzyme has converted a part of the starch into dextrin, which would render the débris more adhesive.

Does the débris from whole-meal give rise to more or less acid than that from white bread?

* British Medical Journal, I9I I, p I3 3 et seq. 
The results of my experiments show that it gives rise to more. For instance:

\section{Substance.}

White-bread débris

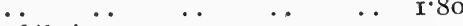

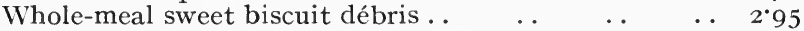

Whole-meal plain, very hard biscuit débris $\quad \ldots \quad \ldots \quad 3^{\circ}$ oo

It has also been shown above that the addition even of 30 per cent. of bran does not prevent acid-formation.

T. G. Read $\uparrow$ is of the opinion that dental caries may be prevented by eating bread from which the germ is not removed, stating that the ferment contained in the germ prevents the formation of lactic acid "during mastication." No scientific evidence is, however, brought forward in support of this view, which is quite at variance with the results of the above experiments.

Does the mastication of whole-neal bread appreciably increase the salivary flow?

In Table I. it will be seen that this is not so. The " brown " bread used there was stated to be whole-meal, but was of no recognized variety. It gave rise to an alkalinity index of $\mathrm{I} \cdot 8$, as compared with dry white bread $2 \cdot 84$, bread and butter (soft) $I \cdot 28$, and biscuit (very slightly sweetened) $2 \cdot 9$.

No definite advantage, therefore, can be claimed for it as a " protection producer."

It remains, therefore, to conclude that whole-meal bread has not been shown to have any advantage over white bread from the point of view either of tooth formation or tooth preservation.

\section{Summary.}

I. That as to their fermentability and lodgeability-the " acid potential "- all carbohydrate articles of diet fall into two well-defined groups:

* Stone-rolled.

$\dagger$ Proceedings of the Royal Society of Medicine, Odont. Section. I912. p. II. 
(a) Those of originally neutral or alkaline reaction, giving rise to the formation of much acid.

(b) Those of originally acid reaction, giving rise to very little or no acid, or a definite alkaline reaction.

2. That by a mixture of strong salivary stimulants with the acid-forming carbohydrates the resulting reaction may be turned from an acid to an alkaline one.

3. That this latter effect may be more certainly obtained by a sequence than by a mixture.

4. That a mixture of protein (as meat) with carbohydrate has practically no effect in reducing the amount of acid formed, but that a sequence of meat after carbohydrate does prevent the formation of acid probably by its detergent action. Such a sequence is, however, quite impracticable and undesirable, and therefore has not been considered.

\section{Conclusions.}

That in order to prevent the retention of fermentable carbohydrates on and between the teeth, and so eliminate or very considerably reduce the carbohydrate factor in the production of caries, starches and sugars should cn no account ever be eaten alone, but should in all cases either be combincd with a substance having a distinctly acid taste, or they should be folloreed by such substances as have been shown to have an "alkaline potential"; and the best of these are, undoubtedly, the natural organic acids found in fruits and vegetables. 


\section{THE REDUCTION OF THE BACTERIAL AND CARBO- HYDRATE FACTORS BY ARTIFICIAL MEANS}

\section{The Use of Antiseptics.}

EVER since the rôle which bacteria play in the production of caries has been recognized, antiseptic drugs have been widely used in the prophylactic treatment of the disease, both by the laity in proprietary articles and in the prescriptions of medical and dental practitioners. This use has been more or less empirical, or, rather, based upon the general principle that where organisms are, there of necessity antiseptics should be also, in order to maintain the health of the tissues.

I do not think it has ever been claimed by anyone that from clinical experience any good whatever has been seen to accrue from their use. It cannot be said that they have checked or arrested the progress of caries in the slightest degree. The mouth is an extremely difficult cavity to deal with by means of antiseptics. When we remember how extremely difficult it is to sterilize the hands even, the whole surface of which can be well scrubbed for a prolonged time, and can be immersed in antiseptics of far greater strength than could be tolerated in the mouth, we can realize how extremely difficult, if not impossible, it must be to obtain a condition approaching sterility in the mouth. It is a cavity made up of nooks, corners, folds, and irregularities, every angle and surface of which is coated with micro-organisms; 
and, as is well known, such conditions offer great obstacles to the action of antiseptics, and favour the development of bacteria. (Even with the utmost aseptic precautions, using a sterile cannula and segregator, I have found it practically impossible to get a sample of saliva free from organisms.)

The cavity is one, too, which is always moist with an albuminous and alkaline fluid, and this tends in the majority of cases to at once combine chemically with the antiseptic and render it ineffective (the potent antiseptic being chiefly the salts of heavy metals). The majority of such antiseptics, too, at once check the action of ptyalin. Moreover, when it is remembered how comparatively infinitesimal is the length of time during which such antiseptics are used, it would be surprising indeed if any beneficial action were to result. The time, as a rule, spent in rinsing the mouth is rarely more than, if as much as, a minute. Supposing this be done three times a day, this makes three minutes in twenty-four hours, or for every minute the antiseptic is acting there are I,437 during which it is not acting; and when we bear in mind the rapidity with which organisms proliferate, it does not seem at all likely that such a short and partial exposure to an antiseptic would result in any permanent lowering of the number of organisms in the mouth.

Certainly the use of antiseptics is not based upon the findings of any scientific investigations.

R. Bessenge* tested the antiseptic value of a large number of mouth-washes, and concluded that hydrogen dioxide Io per cent. alone acted antiseptically in a few seconds; all the remainder required at least four minutes-a length of time which would be impracticable to recommend for the routine use of mouth-washes.

Wadsworth, $\uparrow$ after testing a number of antiseptic mouth-

* Deutsche Medizinische Wochenschrift, August 19, 1909.

$\dagger$ Journal of Infectious Diseases, October 30, 1906. 
washes, such as formalin, lysol, hydrogen dioxide, found that none were so efficient as alcohol 30 per cent., preferably with glycerine and sodium chloride added.

Against this supposed beneficial action of alcohol, however, are the findings of Floxas,* who, after the examination of 729 workmen, found that the percentage of caries in total abstainers was nearly one-third less than in those who took alcohol, the ratio being remarkably constant for all ages from fifteen to fifty. Probably, too, the amount of alcohol taken into the mouth, and the total length of time the oral tissues were bathed in alcohol per diem in the case of those who habitually drank alcohol, would be far in excess of the amount and time which any person would devote to the use of an alcoholic mouth-wash each day. Since the subjects of the investigations were, however, Mohammedans, it is probable that those who were not faithful to the Prophet's teaching indulged in alcohol to excess, and thus produced a vitiated rondition of the gustatory nerves, leading to a decreased salivary secretion.

Hunt $\dagger$ has investigated the inhibitory effect of a number of antiseptics used as mouth-washes. The solutions were used in the mouth for one minute, and cultures were taken from mixed saliva five minutes, and also one hour, afterwards; these were plated out, and the number of colonies counted. It is to be regretted that no control cultures were madei.e., cultures before the use of the solution-so that the actual diminution in each case might be known. However, none of the solutions used produced anything like sterility of the mouth even for five minutes. This coincides with my own observations on the subject. I have on several occasions tested the antiseptic values of mercuric chloride, formalin, and hydrogen peroxide, in such strengths as could be tolerated

* Dental Cosmos, i9io, p. 939.

† Ibiä., October, 1904, p. 818. 
in the mouth without discomfort, and also various proprietary lozenges said to contain formic aldehyde; and none of them, even when used vigorously with the tooth-brush, were capable of producing anything approaching an aseptic condition of the mouth even for five minutes-when the cultures were taken. The colonies were not counted, but thymol was added to each after twenty-four hours, and the turbidity of the broth compared. The only ones which showed any marked lessening of turbidity as compared with their controls were mercuric chloride, I in 2,000 , in a culture taken one minute afterwards, and the hydrogen peroxide (five minutes). The mercuric-chloride five-minutes culture showed a turbidity only just appreciably less than the control.

The following are the averages of Hunt's results:

\begin{tabular}{|c|c|c|}
\hline Solution. & $\begin{array}{l}\text { Number of } \\
\text { Organisms in } \\
\text { 1.C. Saliva after } \\
\text { Five Minutes. }\end{array}$ & $\begin{array}{c}\text { Number of Organisms in } \\
{ }_{6} \text { C.C. Saliva after } \\
\text { One Hour. }\end{array}$ \\
\hline $\begin{array}{lll}\text { Formalin, I: } 200 & \ldots & \ldots \\
\text { Benzoic acid, I : } 200 & \ldots & \ldots \\
\text { Salicylic acid, I : } 200 & \ldots & \ldots \\
\text { Mercuric chloride, I }: 3,500 & \ldots \\
\text { Mercuric chloride, I }: 2,500 & \ldots \\
\text { Mercuric chloride, I }: 2,500 \\
\text { (with a number of other in- } \\
\text { gredients-thymol, menthol, } \\
\text { alcohol, and glycerine) }\end{array}$ & $\begin{array}{r}637,370 \\
19,874 \\
2 \text { I } 4,8 \text { I } 6 \\
20, \text { I I } 5 \\
3,909\end{array}$ & $\begin{aligned} \mathrm{I}, 590,000 & =\times \quad 2 \cdot 5 \\
\mathrm{I} I 0,5 \mathrm{I} 4 & =\times \quad 5 \cdot 5 \\
640,6 \mathrm{I} 6 & =\times \quad 3 \cdot 0 \\
333, \mathrm{I} 8 \mathrm{O} & =\times \mathrm{I} 6 \cdot 0 \\
5,752 & =\times \quad \mathrm{I} \cdot 5 \\
2,458 & =\div \quad \mathrm{I} \cdot \mathrm{I}\end{aligned}$ \\
\hline
\end{tabular}

From this it would appear that the only antiseptic of any material value in the reduction of the number of organisms in the mouth is mercuric chloride, and this only when combined with a number of other substances. The antiseptic value of the other ingredients does not seem to have been tested, so that the prolonged action seen in this case alone may have been due to their use, and not to the mercury.

Miller tested the antiseptic action of a number of drugs used as mouth-washes. His conclusions are all based on the 
time taken by the particular antiseptic to devitalize mouth organisms in vitro; and this being so, the results are not of much practical value, since the conditions in the mouth are so totally different. Everything in the mouth is in favour of the organisms; in vitro everything is in favour of the antiseptic; and although a drug destroyed all the organisms in a test-tube in one minute, it is obvious from preceding remarks that to infer anything like a similar action in the mouth would be extremely fallacious.

There are, moreover, several very strong objections to the use of the more powerful antiseptics as mouth-washes:

I. Many of them are extremely poisonous. Miller and Hunt have both pointed out that the risk of swallowing sufficient to cause toxic symptoms is very remote, and doubtless this is so in adults of average intelligence. It is not, however, with adults so much that we are concerned in the prevention of dental caries-it is with children; and it is not always possible for someone to supervise the cleaning of the teeth, so that there would be great danger of too much being swallowed, as anyone who has watched children cleaning their teeth and washing their mouths out will know.

2. Chassevant and Richet* have shown that in the process of lactic-acid fermentation the addition of metallic salts in very slight degrees of concentration, although having an inhibitory effect upon the micro-organisms, may not interfere with the acid-production at all. My own experience supports this. For instance, if carbohydrate be infected with mouth organisms and lactic acid formed, and if this be then neutralized and strong thymol solution added, the carbohydrate will slowly again become acid, although subcultures show the process to be a sterile one. That is to say, the antiseptic has destroyed the organisms, but not their enzymes.

3. Lastly, and most serious objection of all, most of the * Comptes Rendus, cxvii., p. 673. 
stronger antiseptics are salivary depressants; hence, by diminishing the flow of saliva, they promote stagnation in the oral cavity, remove the natural means of protection, both active and passive, and thus make conditions much more favourable for the organisms. Hunt in his investigations noted this point, but did not attach any importance to it. He says that after using the antiseptic mouth-wash it was ejected, and "the variation in amount between it and the wash taken into the mouth was too small to measure with a graduate." This means, of course, that during the minute when the antiseptic was in the mouth no flow of saliva had occurred. The use of such drugs, therefore, undoubtedly in this respect alone more than counteracts any beneficial action they may have as disinfectants, and, if habitually used, may do far more harm than good.

NotE.-The above only relates to antiseptics used for the prevention of caries. Undoubtedly, in acute inflammatory conditions of the mouth antiseptics are indicated and necessary; their usefulness, though, is increased by being used in conjunction with salivary stimulants.

\section{The Use of the Tooth-Brush.}

That the teeth may remain perfectly healthy without the use of a tooth-brush is evidenced in the case of many " native " races, and also in isolated cases in higher civilized races. On the other hand, many uncivilized peoples assiduously clean their teeth with a pointed stick frayed out at the end, and it is noticeable that this occurs in those races whose normal diet is less acid and softer than that of others-i.e., Kaffirs and some Hindus.

The food of modern civilized peoples is essentially soft and little acid; and since it has been shown that such foodstuffs not only adhere more to the teeth and produce more acid, but also fail to evoke the " natural " agencies of protection 
(since they are not "natural" stimuli), it would seem only reasonable that some artificial means should be adopted to promote the more rapid removal of such débris from the teeth.

That by means of the tooth-brush a considerable amount of fermentable material may be removed from the teeth is proved by the experiments on the relative lodgeability of various foodstuffs. Even if, as is quite possible, all the débris is not thus removed, it is obvious that it must considerably reduce the amount capable of being transformed into acid, and so make it possible for the saliva to deal with the remainder.

The following experiments bear on the question:

I. The teeth being unbrushed for twenty-four hours, bread was eaten, and five ninutes afterwards the débris was collected by brushing the teeth, and incubated in the manner previously described. In twenty-four hours $0^{\prime} 3\left(\frac{\mathrm{N}}{5} \mathrm{NaOH}\right)$ units of acid were developed. Half an hour after the brushing bread was again eaten, and the débris collected and incubated. At the end of twenty-four hours only O.I5 acid units had developed.

2. The teeth being uncleaned for twenty-four hours, bread débris was collected in the usual way, and neutral litmus added. In two hours it became distinctly acid. The same procedure was carried out a few minutes after cleaning the teeth, and the incubated débris did not become acid for five hours.

Other experiments were confirmatory of these results. We may therefore assume- $(a)$ that the tooth-brush does remove a considerable amount of acid-forming débris; $(b)$ that the tendency for certain foods to lodge on brushed teeth is less than on unbrushed teeth, for the total amount of acid formed was only half in the former case what it was in the second, and the tinie required to become acid was more than double. 
The form of tooth-brush generally used, and the manner of using it, as a rule leave much to be desired. The brush must be a small one; the bristles should be neither too hard nor too soft, and should be of unequal lengths; the handle should preferably be bent at an obtuse angle away from the bristles. Such a brush is shown in Fig. 72. With such a brush all

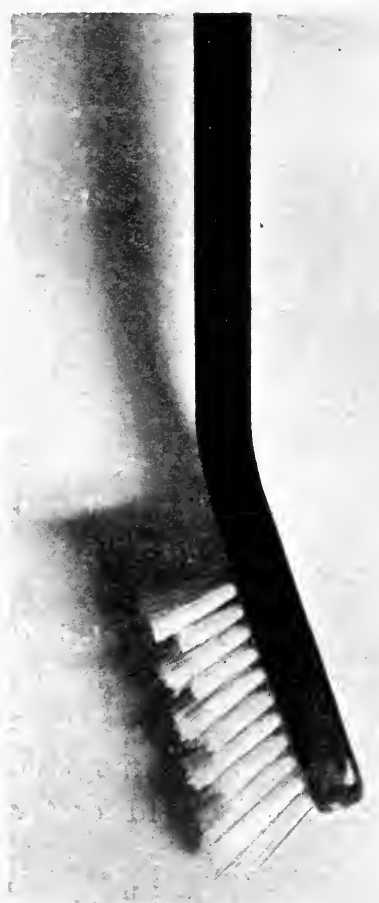

Fig. 72.-TOOTH-BRUSH. surfaces of the teeth can be reached, and only about two teeth can be brushed at the same time This is a very essential point, since it necessitates a little longer time being spent on the operation. But most important of all is the manner of brushing. As a rule teeth are not brushed-they are rubbed and scrubbed. Food débris is simply forced into the interdental spaces, and the imbrications of the enamel are likewise filled up. The error arises through a non-appreciation of conditions and structure. Let a person thoroughly grasp the fact that the surface of a tooth is not smooth, but "rough" or corrugated; that it is covered by a tough membrane; that what it is required to remove has not soaked into the teeth, but is lying against them; and he will naturally and unconsciously adopt the right kind of action-i.e., he will brush the teeth. (To give a homely simile, the teeth should be brushed after the manner that a clothes-brush is used, rather than as a scrubbing-brush is.) Such brushing is only 
possible with a very small brush. The name " tooth-brush" as applied to articles long enough to reach from the canine tooth to the last molar is a misnomer; there cannot possibly be enough room in the mouth for their use in "brushing." The more prominent parts of the teeth only can be scrubbed, and by no means can the food débris be brushed off the teeth as it should be.

Instructions, then, should be given to brush the teeth systematically. The mouth should be held wide open, and one should commence at the last molar of one jaw, coming forward to the canine region, then returning along the opposing teeth. Next, the other side is treated similarly, and lastly the incisors. Not more than two teeth at a time should be brushed, and this should be done both horizontally and vertically (away from the gums) for the buccal and labial aspects, and forwards and sideways for the occlusal surfaces. Such instruction may sound rather tedious, but in practice it is exceedingly simple; the habit is soon acquired, and the total time, including several cleansings of the bristles, need not exceed two or three minutes. (This perhaps sounds a very short time, but it will seem a rather long one to many people.)

It is obvious that under modern conditions of life the toothbrush cannot be relied upon alone to prevent caries. If it could be used assiduously, and in the manner described, inmediately after every meal, this might be so, but in the vast majority of cases such a suggestion would be impracticable.

The tooth-brush should rather be regarded as an adjuvant to other means of prophylaxis; and if, as is most frequently the case, it is used only in the morning, as something which will lessen the tendency of foodstuffs to lodge against and between the teeth.

The brush should of course be kept clean, either by per- 
manent immersion in a weak antiseptic solution or by being washed daily with soap and water.

D. D. Smith of Philadelphia has of recent years elaborated what may be termed the apotheosis of mechanical prophylaxis. Patients are required to present themselves at least once a month, when each tooth is individually subjected to a vigorous cleansing and polishing on every surface by the use of wood points and pumice. The results are said to be excellent. This is doubtless brought about by the decreased tendency of foodstuffs to lodge on highly polished surfaces. Smith claims that the mechanical stimulus brings about a reaction on the part of the pulp, causing solidification of the dentine and enamel; but, so far as I know, he has not attempted to show how lime salts may pass from the pulp to the enamel surface, and any solidification of the dentine is absolutely immaterial. In fact, according to the views which we have advanced as to the post-eruption hardening of enamel, Smith's treatment would probably prevent such action taking place.

The method may be regarded as one which may prevent dental caries, but as one which is extremely limited in its application. Probably not I per cent. of those afflicted with caries could afford it; and, as Miller has pointed out, those who do submit to it are patients who would otherwise be very careful in their attention to the teeth at home.

Another school-those dentists practising what are known as Black Club methods--are enthusiastic advocates of the prevention of caries by the excision of all vulnerable areas in susceptible teeth and replacing them with gold-a method which, if followed to its logical conclusion, should lead to the gold encasement of half the teeth of civilized peoples. To say that because caries has attacked one spot in the surface of a tooth, therefore the total destruction of that surface is inevitable unless the whole of it is excised, is merely a confession of failure to prevent caries by more rational or physiological means. 


\section{The Use of Dentifrices.}

It has been shown previously that the great majority of dentifrices are salivary depressants, in that they are composed chiefly of chalk, and therefore alkaline in reaction. Undoubtedly the majority of dentifrices are far more strongly alkaline than is saliva, and if they did not interfere with the salivary secretion their use could not be other than beneficial; but this is not so. What occurs is that the mouth for a minute a day is rendered highly alkaline, but this is brought about at the expense of lessening the amount and alkalinity of a constantly flowing natural mouth-wash; and there can be no doubt that for the purpose of neutralizing acids which are slowly but continuously being formed a continuous alkaline flow is much more efficacious than the application of a comparatively strong alkali for about one minute, or at most two, in twentyfour hours. We are therefore driven to the inevitable conclusion that alkaline dentifrices and mouth-washes for the prevention of dental caries must be abandoned, and we further conclude that some substance which is a salivary stimulant should be used in order to promote and educate the activity of the salivary glands. Now, it has been also conclusively proved that the best substances for this purpose are acids, and there is not the slightest reason why the best should not be used in this case.

The objection will probably be at once raised that acids are "bad for the teeth," in that they can be shown to decalcify them; but this is one of the fallacies of experiments conducted entirely in vitro. It has been shown that comparatively weak acids are the best salivary stimulants; and even though they should cause some superficial decalcification (which it will be shown they would not), yet if at the same time they prevented caries from occurring, it would be infinitely the lesser of the two evils. For the evil would be manifested probably 
as erosion, attrition, and abrasion; but these would be slower in progress and infinitely less in prevalence than caries, and, moreover, the children would escape-which is the very gist of the problem with which we are dealing. But would acids cause any decalcification when used in this way? We have already seen that five and fifteen minutes after their use the alkalinity index of the mouth is always considerably increased above normal, and that in the case of as strong an acid as citric in the lemon, two minutes after its use, and with the inclusion of small fragments of lemon, the saliva was intensely alkaline. This may be readily tested roughly by anyone with a piece of litmus-paper placed in the mouth or against the teeth half a minute after eating or washing the mouth out with anything weakly acid. Undoubtedly, there is a short time during which the acid remains unneutralized, but this is not sufficient to cause any effect, except, perhaps, on the occlusal surfaces in the case of masticating acid substances which are actively pressed and ground between these surfaces.

From a very large number of observations, I am convinced that no harm will accrue to the teeth from the use of those acids, in such strengths as they are active salivary stimulants. An acid substance which it is suggested should be used in a general manner for this purpose is acid potassium tartrate. This substance has several advantages:

I. It is an active salivary stimulant.

2. It is a form of acid which is widely distributed in Nature, and one, therefore, to which the salivary glands have by the process of evolution been adapted.

3. Its solubility is only I in 200 of water, and, therefore, if it be used in the liquid form there can be no danger of its being used in too strong a solution, either on account of faulty making-up or of subsequent evaporation.

4. It has an agreeable taste, which may be made more so by the addition of saccharine. This is, again, an ex- 
tremely important factor in applying these principles to children.

In the laboratory, sclerotic and malacotic teeth have bein actively scrubbed daily for a period of six months with a saturated solution of acid potassium tartrate, without in any case producing the slightest sign of any decalcification, or abrasion even. The teeth after the scrubbing were kept in water at about neutral point, whereas in the mouth they would have been immediately bathed in a much stronger alkaline fluid.

We have already shown, too, how the addition of this salt to saliva is beneficial in precipitating the mucin and in favouring dialysis. Its addition to saliva causes the latter to become watery, and well adapted to flowing and percolating between the teeth and into fissures and grooves; whereas the addition of a similar amount of carbonate of soda causes the saliva to become thick and viscous, and eminently unsuited for its function-in fact, promotes stagnation instead of circulation. Moreover, should any acid potassium tartrate remain in the saliva, it seems to undergo an alkaline decomposition. For instance, 2 c.c. of saliva made faintly acid by the addition of this salt were incubated; in twelve hours it was faintly alkaline, and in forty-eight hours it showed an alkalinity of $I \cdot O\left(\frac{N}{50}\right)$. Again, to Io c.c. of a mixture of glucose and saliva, acid potassium tartrate was added until it showed an acidity of 5 units per c.c. It was then incubated for two days, when the acid units present were found to be only 0.22 per c.c. This rapid reduction of its acidity in the presence of mouth organisms has been repeatedly observed; so that, in the extremely unlikely case of any remaining in the mouth unneutralized, it would become progressively less acid, and finally alkaline. Such a mouth-wash as has been indicated should be used to rinse the mouth thoroughly in the first place. The teeth should then be brushed. It is sufficient in the case of children 
to use the tooth-brush with water alone, then to rinse the mouth again thoroughly with the acid-sweet wash; this will cause a rapid secretion of alkaline saliva, which should be, by the action of the cheeks, tongue, and closed lips, forced through the dental interstices. The mouth should always be rinsed out in this fashion both before and after brushing, in order to clear the approximal surfaces of the teeth; this is facilitated by the presence and direction of the imbrications on the surface of the enamel, guiding and directing fluid in horizontal channels between the teeth, whereas their presence actually hinders the use of a tooth-brush, since the latter can in this situation only be used in a vertical direction-i.e., at right angles to the imbrications. Floss silk would be an excellent material for clearing the imbrications if it could be used, but in the majority of cases it cannot be, on account of the difficulty of passing it between the teeth; this material is, I think, to be regarded as indicated only in quite special cases.

The following are examples of formulæ which may be employed as acid mouth-washes:

A.

R Potass. tart. ac. Sodii chloridi . . Glusidi Aquam

I3.

C.

D. Be Potass. tart. ac. $\quad \ldots \quad \ldots \quad \ldots$ gr. ii.

$$
\begin{array}{lccccc}
\text { R Potass. tart. ac. } & \ldots & \ldots & \ldots & \text { gr. ii. } \\
\text { Ac. tartarici } & \ldots & \ldots & \ldots & \ldots & \text { gr. } i . \\
\text { Glusidi } & \ldots & \ldots & \ldots & \ldots & \text { gr. } \frac{1}{4} . \\
\text { Aquam } & \ldots & \ldots & \ldots & \ldots & \text { ad } 3 \text {. }
\end{array}
$$

$\begin{array}{cccccc}\text { C. Ac. tartarici } & \ldots & \ldots & \ldots & \ldots & \text { gr. ii. } \\ \text { Quin. sulph. } & \ldots & \ldots & \ldots & \ldots & \text { gr. } \frac{1}{4} \text {. } \\ \text { Glusidi } & \ldots & \ldots & \ldots & \ldots & \text { gr. } \frac{1}{4} \text {. } \\ \text { Aquam } & \ldots & \ldots & \ldots & \ldots & \text { ad } 3 \text { i. }\end{array}$

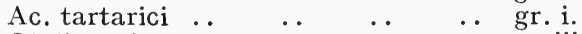

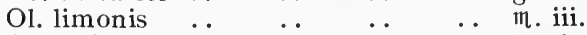

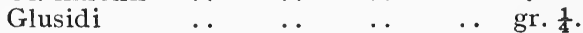

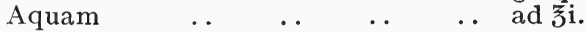

Of the above, the last (D) is the most pleasant combination, and the one containing quinine least so; the first one (A) 
possesses a bland and soothing effect, and would probably be efficacious in catarrhal conditions of the mucous membrane. These formulæ are only suggestions, and may be modified in various directions to suit special cases or "tastes." The saccharine (glusidum) and the tartaric acid may in some instances, perhaps, be reduced by one-half. In order to ascertain the action and effect of such mouth-washes, the above series was tested in the following manner: The acidity of Io c.c. of the solution was first estimated in terms of $\frac{\mathrm{N}}{50} \mathrm{NaOH}$; the mouth was then washed out for one minute with Io c.c. of solution, and the amount expectorated was measured. Five minutes afterwards the "resting" saliva was estimated for amount and alkalinity per minute. The results are expressed in the following table:

\begin{tabular}{|c|c|c|c|c|c|c|c|c|}
\hline & & & \multicolumn{3}{|c|}{$\begin{array}{l}\text { Variations produced in the } \\
\text { Solutions. }\end{array}$} & \multicolumn{3}{|c|}{$\begin{array}{l}\text { Resting Saliva Five Minutes } \\
\text { after using Mouth-Wash. }\end{array}$} \\
\hline & & & $\begin{array}{l}\text { Original } \\
\text { Acidity of } \\
\text { 1o C.C. }\end{array}$ & $\begin{array}{l}\text { Acidity } \\
\text { on Expec- } \\
\text { toration. }\end{array}$ & $\begin{array}{c}\text { Amount } \\
\text { expector- } \\
\text { ated in C C. }\end{array}$ & $\begin{array}{l}\text { C.C. per } \\
\text { Minute. }\end{array}$ & $\begin{array}{l}\text { Alkalinity } \\
\text { per C.C. }\end{array}$ & $\begin{array}{c}\text { Alkalinity } \\
\text { per } \\
\text { Minute. }\end{array}$ \\
\hline $\begin{array}{l}\text { A. } \\
\text { B. } \\
\text { C. } \\
\text { D. }\end{array}$ & $\begin{array}{l}\ldots \\
\cdots \\
\cdots\end{array}$ & $\begin{array}{l}\ldots \\
\ldots \\
\cdots\end{array}$ & $\begin{array}{l}0 \cdot 90 \\
2 \cdot 00 \\
3 \cdot 40 \\
2 \cdot 25\end{array}$ & $\begin{array}{l}O \cdot 40 \\
I \cdot I O \\
I \cdot 30 \\
0 \cdot 65\end{array}$ & $\begin{array}{l}12 \cdot 0 \\
12.6 \\
13.0 \\
14.0\end{array}$ & $\begin{array}{l}2 \cdot 5 \\
2 \cdot 4 \\
2 \cdot 7 \\
2 \cdot 9\end{array}$ & $\begin{array}{l}\mathrm{I} \cdot 00 \\
\mathrm{I} \cdot \mathrm{O} 6 \\
\mathrm{I} \cdot 24 \\
\mathrm{I} \cdot 28\end{array}$ & $\begin{array}{l}2 \cdot 50 \\
2 \cdot 50 \\
3 \cdot 34 \\
3 \cdot 70\end{array}$ \\
\hline
\end{tabular}

It is therefore clear that the acidity of such solutions is rapidly diminished whilst being used, and that they subsequently give rise to a very much increased alkalinity index. One minute is possibly longer than they would be retained in the mouth in actual use, and probably two half-minutes with an interval between would be more efficacious.

It will be noticed that in all cases the amount of fluid expectorated was greater than that taken in, showing an increase of from 2 to 4 c.c.--that is to say, that during the minute that the solution was in the mouth salivary secretion was actively going on. This is in striking contrast to what 
Hunt found during the use of antiseptics (see p. 270). As a control, distilled water was tested in a similar manner, and in all cases the amount of fluid in the mouth became less; this is a curious phenomenon which I do not think has been observed before, and is probably due to osmosis, the water passing into the cells of the mucous membrane of the mouth. Several individuals have been tested on several occasions, and always with the same result, the diminution varying from I to 3 c.c. This would seem to suggest that when it is required to obtain the full therapeutic effect of a drug on the oral mucous membrane, it should be in such strength that the solution will have a lower endosmotic equivalent than saliva.

The use of the mouth-wash will, of course, be most efficacious at night-time, since it will not only remove the greater part of the carbohydrate débris from the mouth, but will promote an increased secretion of saliva with strong alkaline and diastatic properties, which will be more than sufficient to deal with any carbohydrate remainder before the organisms have the opportunity during the night of converting it into acid.

In cases where there would appear to be a tendency to rapid caries, the use of such a mouth-wash should be insisted on at least three times a day. If the extreme seriousness of the condition be explained to parents, there will be no more difficulty about this than in enforcing the "taking of medicine" during the course of any other disease; in fact, there will be considerably less difficulty, since it is very pleasant to the taste. (I have found that for this reason, as a general rule, children are more keen to remember its use than are their parents.) The swallowing of such solutions is, moreover, not at all harmful, and may be beneficial. On the contrary, the constant swallowing of chalk from alkaline dentifrices may very probably be one of the causes of the chronic constipation so prevalent at the present time.

In the cases of adults, especially where there is any tendency 
to staining of the teeth or the deposit of calculus, acid potassium tartrate in the form of a fine powder may be used on the brush very occasionally, provided that the teeth are brushed and not scrubbed, or, no doubt, marked abrasion might soon result. The use of the solid salt also is immediately followed by a rapid secretion of saliva, which should likewise be forced through the dental interstices.

As we have seen previously, these mouth-washes act in the manner indicated by reason of their stimulating the gustatory nerves of the tongue. It may therefore not be out of place to refer to a description of the action of chemical substances on the tongue by J. B. Haycraft.* The latter author states: "When a sapid substance acts upon the tongue, the taste may be followed by after-effects which have been compared with the after-images in the organ of vision. M. v. Vintschgau describes the metallic bitter of the taste which sometimes remains subsequent to the breaking of a current through the tongue, which, during its passage, produced a metallic acid sensation.

" Of greater interest, however, is the after-effect produced by a sapid substance itself. It has long been known that after tasting common salt the tongue is more sensitive to the taste of sugar; so much so, that a very weak solution of sugar-too weak to be distinguished as a sweet liquid-produces a taste, if it is taken after washing out the mouth with salt and water. In the same way, acid stimuli render the tongue more susceptible to sweet substances, and after tasting weak sulphuric acid even distilled water appears somewhat sweet. Not only does common salt produce after-susceptibility to sweet, but many other substances act in the same way.

"Simultaneous contrasts also appear to be capable of demonstration in the case of taste as in the case of vision. If a sapid substance be applied to one region of the tongue,

* Schäfer's "Textbook of Physiology," vol. ii., p. I245. 
other parts of the organ are rendered more susceptible to other stimuli. Thus, if one border of the tongue be rubbed with salt, the other border will give a reaction with sugar out of all proportion to the strength of the stimulus employed. Salt and acid give likewise simultaneous contrast; but it is questionable whether any facts can be brought forward to show that bitter sensations are affected by other stimuli simultaneously applied."

Of course, other acids or acid salts may possibly be used in a similar manner. There are, however, two dangers to be guarded against-those of using an acid either too weak or too strong. If an acid be used in too weak a solution, it will certainly do much more harm than good, for it will reduce the alkalinity of the mouth without exciting any increased flow of saliva, and thus for. some time the reaction of the mouth may be actually acid; or the weak acid, by being unneutralized too long, may in a stagnating cavity attack the enamel of the teeth itself. This is evidently why the lactic acid of fermentation is not neutralized-it is formed in such small quantities that the gustatory nerves are not stimulated. If an acid be used in too strong solution, it may have a precisely similar effect: the stimulus to the gustatory nerves will be too great; it will have a paralyzing effect, and the flow of saliva be consequently diminished, giving the opportunity to the stronger acid to directly attack the teeth.

Acid mouth-washes will also, I think, prove to be beneficial in reducing the number of acid-forming organisms in the mouth; for, as is well known, those organisms which form acid are very sensitive to the action of acids.* Moreover, all organisms are very sensitive to sudden changes in the composition and specific gravity of the media in which they are growing, and such changes are brought about in the saliva by the action of weak organic acids.

* Oppenheimer: "Ferments and their Action," p. 237; Cohn, Zeitschrift f. Physiol. Chemie, xiv., 75. 
The use of such mouth-washes will also tend to keep the calcium phosphate and carbonate in solution, instead of their being precipitated upon the teeth in the form of calculus, which, as is well known, sooner or later leads to a serious condition of oral sepsis. On the other hand, the use of chalk dentifrices frequently promotes the formation of calculus by particles remaining against the teeth and acting as a focus for precipitation of lime salts from the saliva.

A correct physiological method, however, of preventing such deposition would be to increase the carbon-dioxide content of the saliva, since it is owing to a deficiency of this gas or its rapid escape from the saliva that the precipitation of the lime salts occurs. Exactly in what ways this may be done is not at present clear; but this much is quite probablethat the amount of carbon dioxide in the saliva is dependent upon muscular metabolism; and that the carbon dioxide is derived from the blood-stream and is not manufactured in the glands, since during stimulation the blood flowing from the glands assumes a bright-red arterial colour. Therefore muscular activity of the body generally should lead to a decreased tendency towards deposits of calculus. Clinical experience lends at least a negative support to this, for such deposits are usually seen in two classes of people:

I. Those in whom there is a localized oral stagnation owing to loss of function.

2. Those in whom salivary secretion has been normal and has protected the teeth during early life, but in whom, when the more sedentary period of life has arrived (causing, presumably, a decrease of carbon-dioxide formation and excretion), the lime salts have been precipitated. That is to say, the alkalinity and phosphatic indices may remain normal, but their solvent carbon dioxide has diminished to its minimum point. 


\section{Influence of Tobacco Smoke.}

Miller showed that, in vitro, cigar smoke has a very marked antiseptic action on mouth organisms. This was confirmed by Tassinari.

Again, however, one sees the fallacious character of wholly in-vitro experiments, for clinically one only too frequently observes marked and abundant caries in the mouths of inveterate cigarette smokers. I am inclined to think, however, that pipe and cigar smoking in moderation may produce some degree of immunity. The difference, I think, lies in the fact that, even in moderation, cigarette smoking tends to depress the salivary glands; whilst with a pipe or cigar the flow is, up to a certain point, certainly increased. This effect is aggravated by the fact that cigarette smoking is much more frequently carried to excess than either pipe or cigar smoking, which frequently occurs also at a time of life coinciding with increased caries susceptibility.

Again, different tobaccos seem to have the power of selecting different areas of the mouth, tongue, and throat for stimulation; and it probably depends upon the area stimulated in any particular individual, and the intensity of it, what the effect will be both on the organisms and on the salivary secretion. 


\section{THE INOCULATION OF THE MOUTH WITH OTHER ORGANISMS}

THE mouth might be inoculated* with the definite purpose of either (I) introducing organisms which should exert an antibiotic effect upon the established organisms; (2) introducing organisms which would decompose carbohydrates, with the formation of alkaline products; or (3) introducing organisms which would rapidly remove the carbohydrate débris before lactic acid could be formed.

I. As regards the first possibility, although the forces of antibiosis are known to exist-in fact, must exist-in the mouth to a very large extent, it seems (at present) to be the rule that "pathogenic" organisms are the ones which are crowded out or succumb, and that the organisms of caries are the ones which survive. We do not yet know enough about the biological characters of mouth organisms alone or in combination, in the mouths of immune individuals and of susceptible individuals, to be able to say which, if any, are inimical to the acid-forming group responsible for the primary decalcification of enamel; or whether any organisms exist which, if introduced into the mouth, could be induced to establish themselves and exert such an action. This is a subject which will probably be shown to have possibilities when it is thoroughly worked out.

2. As regards the second possibility-the introduction of

* The word "inoculate" is used here, not to mean hypodermic inoculation, but simply the insertion of organisms in the oral cavity preferably by their being rubbed between the teeth. 
organisms which might from carbohydrates produce alkaline end-products--there are two classes of bacteria which might conceivably bring this about.

Firstly, some of the hyphomycetes (moulds). It has been observed on many occasions that in the experimental production of caries, when moulds establish themselves in the bread-and-broth mixture, the reaction becomes alkaline. Attempts have therefore been made to isolate the organisms responsible for such a change-so far, however, quite unsuccessfully.

The second class is the mesenteric group of organisms. These, as has been well known at least since Goadby* some years ago carefully worked out their biology and cultural characteristics, in a carbohydrate medium produce an alkaline reaction.

It seemed, therefore, at first that in this group were organisms well adapted to the purpose of neutralizing the effects of the lactic-acid-forming organisms, and some work was undertaken along such lines. It has, however, at any rate for the present, been abandoned for the following reasons:

(I) Although an alkaline reaction can be obtained when the organism is in pure culture, yet when it is mixed with anything approaching a " normal " amount of acid-forming organisms the reaction is always acid; that is to say, the acid-forming group seems to be the more vigorous, and able largely, if not entirely, to suppress the carbohydrate decomposition produced by the mesenteric group.

This, indeed, is what one would expect, seeing that the mesenteric group of organisms is widely distributed, both in milk and water, and they are practically always present in the mouth, presumably partly by inoculation and partly by becoming established. It would therefore appear to be obvious that under ordinary conditions the mesenteric group

* "Mycology of the Mouth," London, 1903. 
cannot obtain the upper hand, since the reaction produced in carbohydrates by mixed mouth organisms, from immunes as well as susceptibles, is always acid. Until, therefore, we can by some means definitely reduce the vitality of the acid-forming organisms without affecting the mesenteric bacilli, inoculation with such organisms would seem to be useless.

(2) The organisms are powerfully proteolytic; in fact, they are the ones which are largely responsible for the digestion of the collagenous matrix of the dentine. In view of this fact and of the important rôle which has been assigned to Nasmyth's membrane, it does not seem wise to employ organisms which would destroy the latter structure. (Especially would this be likely to occur in positions where the membrane is not removed by attrition, and where, therefore, its presence is required most.)

3. The Introduction of Organisms which rapidly break up the Carbohydrate Débris, with the Formation of Innocuous Substances.-Mariy of the higher organisms-hyphomycetes and blastomycetes - have the property of transforming certain carbohydrates into carbon dioxide and alcohol.

The commonest and most familiar example of this is the fermentation of sugars by yeast. The equation which expresses in general terms the action which takes place is-

$$
\mathrm{C}_{6} \mathrm{H}_{12} \mathrm{O}_{6}=2\left(\mathrm{C}_{2} \mathrm{H}_{5} \cdot \mathrm{OH}\right)+2 \mathrm{CO}_{2} \text {. }
$$

This does not express exactly what occurs under all circumstances; a certain proportion of the sugar, for instance, is consumed and assimilated by the organisms, and another portion is converted into by-products. These are, however, in all cases trifling in amount, and consist of glycerine and traces of succinic and acetic acids, the latter not amounting to more than 0.7 and 0.05 per cent. respectively of the total quantity of sugar transformed into alcohol and carbon 
dioxide.* More by-products are, however, produced by the mucor species than by the saccharomyces group.

There seems to be no reason why some variety of the saccharomyces group should not be utilized for the purpose of breaking up the carbohydrate débris in the mouth into carbon dioxide and alcohol. Various forms of saccharomycetes are occasionally found in the mouth, but there is no evidence to prove that they exert any harmful action, except in the case of Saccharomyces albicans, the organism of thrush, and then only in weak, debilitated individuals.

Goadby $\uparrow$ has noted the presence of a yeast in a very few cases of pyorrhœa alveolaris, but of what particular variety is not mentioned, except that in two cases it was pathogenic for guinea-pigs. But, as the same observer has also shown, the infections in such cases are always very mixed ones, and it is not suggested that all the organisms found are causative factors; moreover, it is recognized that pyorrhœa alveolaris is usually found in mouths relatively free from caries.

Miller $\ddagger$ has noted the presence of saccharomycetes in the mouth, and stated that he regarded them as "the most harmless of all mouth parasites."

It is now known that yeasts may be beneficially given internally (the dose being 5 to Io grammes in milk) in order to increase the opsonic power of the blood, and to promote a leucocytosis; and boils have been successfully treated in this manner. If, then, it can be shown that opsonins pass into the saliva, the administration of yeast may be of indirect advantage in raising the "opsonic index of the saliva."

Saccharomycetes as a class require for their nourishment (besides carbohydrate) water; a source of nitrogen, preferably in the form of albumin; and various inorganic substances,

* Oppenheimer: “Ferments and their Actions," p. 248 et seq.

$\dagger$ "Mycology of the Mouth," p. I78; Erasmus Wilson Lecture British Dental Journal, I907, p. 895.

$\ddagger$ "The Micro-Organisms of the Human Mouth," p. 345 . 
such as iron, potassium, magnesium, phosphorus, and possibly sulphur.*

The saliva as a medium practically fulfils these requirements. It has, too, been shown by Harden and Young $\uparrow$ that the alcoholic fermentation of glucose is carried on more rapidly in the presence of phosphates, of which saliva contains an appreciable quantity.

Moreover, for promoting the solution of starch débris in the mouth there would seem to be a particular fitness in combining a saccharomyces with the saliva. Morris ${ }_{+}^{+}$has shown that ungelatinized starch granules submitted to the simultaneous action of diastase and yeast may have three times as much dissolved from them as by diastase alone.

The variety of organisms which I think is most suited to the purpose under consideration is Saccharomyces coagulatus. This organism occurs " wild," mixed with moulds and torulæ growing on wet wood surfaces; it is found also mixed with other yeasts, such as are used by brewers and bakers.

I have obtained it in pure culture by the following means: A sample of mixed yeast is obtained and examined to see if it contains bacteria as well as saccharomycetes. The former may be got rid of by the usual method of plating out at room temperature on glucose agar or gelatine to which ro per cent. glucose has been added. The yeast may also be purinied by taking successive subcultures in Io per cent. glucose to which a trace of bouillon has been added and which is kept at room temperature. This favours the development of the yeast, but not of the bacteria. The yeast having been obtained in a practically pure state is heated to $50^{\circ} \mathrm{C}$. for one hour, at which temperature all other varieties but $S$. coagulatus are destroyed. Subcultures are now taken, and a pure culture should result.

* A. Mayer, quoted by Oppenheimer, loc. cit., p. 209.

$\dagger$ British Medical Journal, 1909, p. I376.

$\ddagger$ G. H. Morris, Journal of the Chemical Society, vol. 1xxix., p. 1085. 
$S$. coagulatus is spherical to oblong in shape, and is somewhat smaller than ordinary yeasts, being about 5 to $6 \mu$ in diameter (Fig. 73). It does not grow very rapidly, and in fluid media does not cause turbidity; the whole of the growth is either attached to the side of the glass tube or is in a compact mass at the bottom. Gentle shaking of the tube causes no turbidity. Vigorous shaking is necessary in order to dislodge the colonies; even then they are in obvious particles, and, like

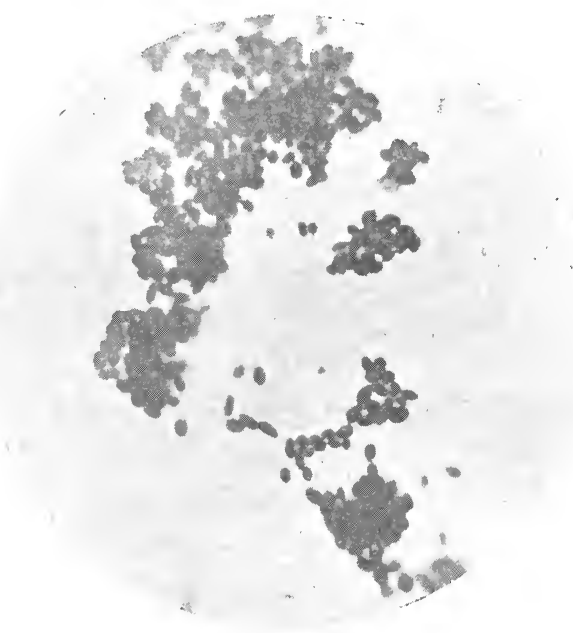

Fig. 73.-Saccharomyces Coagulatus.

a heavy precipitate, soon settle to the bottom, the liquid becoming clear again. This behaviour is quite sufficient to distinguish a pure culture of it from nearly all other organisms.

It is difficult to obtain any of the growth on the platinum needle, and it is somewhat resistant of emulsification on the slide, in this respect reminding one of Staphylococcus viscosus. It acts well as a ferment at body temperature, and can carry 
the fermentation of a solution to a very advanced pointfarther in some cases than S. cerevisia is able to do.*

Cultures of the organisms may be spread out on a filterpaper and dried (avoiding contamination). Their vitality is thereby somewhat lowered, but is soon regained when placed in a culture medium.

The dried cultures may be pulverized, and the mouth inoculated with the powder. At present I have used only about Io grains, but I think probably this dose may be considerably increased with advantage: It has a distinctive but not unpleasant flavour.

The following experiments, amongst others, have been made with Saccharomyces coagulatus in order to determine its possible beneficial action in the mouth.

\section{The Rapidity of Consumption of Carbohydrates.}

(a) Two tubes, A and B, containing ro per cent. glucose medium, were inoculated with saliva and saccharomyces respectively. The tubes were connected with graduated testtubes inverted in water, and were then hermetically sealed. The whole was then placed in the incubator for three days, by which time--

Tube A (saliva culture) had formed $\mathrm{I} \cdot 6$ c.c. of gas.

Tube B (yeast culture) had formed 10.2 c.c. of gas.

That is to say, that a considerable amount of carbohydrate had been used up in B to form carbon dioxide which might otherwise have been utilized for the formation of lactic acid.

(b) Two tubes, A and B, each containing 0.5 gramme of glucose equally diluted, were inoculated respectively with one loopful of "materia alba " and one loopful of the yeast, and were connected to the gas-collecting apparatus. The following

* Matthews: "Alcohnlic Fermentation," p. 30. 
gas formation and sugar consumption occurred in forty-eight hours:

\begin{tabular}{|c|c|c|c|c|}
\hline \multicolumn{3}{|c|}{ Tube. } & \multirow{3}{*}{$\begin{array}{c}\text { Gas Formation. } \\
\begin{array}{r}2 \text { c.c. } \\
2 \text { I , }\end{array}\end{array}$} & \multirow{2}{*}{ Sugar Consumption. } \\
\hline A (mouth & organisms) .. & . & & \\
\hline $\mathrm{B}$ (yeast) & $\ldots$ & . & & $0 \cdot 390 \quad$, \\
\hline
\end{tabular}

In $\mathrm{B}$ tube, therefore, in forty-eight hours $7^{8}$ per cent. of the total sugar had been fermented, whilst in A tube only 9 per cent. had been used up; and in B tube ten times as much was converted into gas as in A. These volumes of gas are only relative, and not absolute, since a good deal was absorbed by the medium and the water over which it was collected.

This absorption of $\mathrm{CO}_{2}$ by the medium causes it to have an acid reaction to litmus, but that this is negligible as a decalcifying agent was proved by the fact that teeth kept in such a medium for one week showed no sign of decalcification of the enamel.

Nevertheless, in impure cultures of yeast with other organisms various acids are formed, especially in vitro, by the decomposition of the retained alcohol; acetic acid is the one chiefly formed, according to the equation-

$$
\mathrm{CH}_{3} \cdot \mathrm{CH}_{2} \cdot \mathrm{OH}+\mathrm{O}_{2}=\mathrm{CH}_{3} \cdot \mathrm{COOH}+\mathrm{H}_{2} \mathrm{O} \text {, }
$$

acetaldehyde being an intermediate product.

This, however, is extremely unlikely to occur in the mouth, especially if there be a good salivary circulation, since the alcohol, being an extremely light, diffusible, and volatile liquid, would rapidly disperse, and be carried away in the saliva or evaporated.

The following experiments, too, show that it would be extremely unlikely to be retained. 


\section{The Dispersion of the Carbohydrate Débris by the $\mathrm{CO}_{2}$ Formation.}

In order to measure the rapidity of the action, short pieces of quill tubing, 0.5 centimetre in length, were filled with mixtures of carbohydrate and organisms coloured with litmus, and suspended horizontally by means of threads in 0.45 per cent. sodium-chloride solution (to represent saliva). They were then placed in the incubator, and the time taken for the short tubes to become empty was observed.

(a) Biscuit was insalivated, and tube A filled with the mixture. To the remainder of the mixture (about 0.5 c.c.) one loopful of $S$. coagulatus was added and thoroughly mixed. Tube $\mathrm{B}$ was then filled with the fresh mixture, and both wore hung in the salt solution and incubated.

Tube A remained full, although strongly acid, for ten days. Tube $B$ was emptied in twenty-six minutes (this being the average of six observations).

Tube $\mathrm{B}$ was filled again with a similar mixture, but containing half the amount of yeast. It was empty in three hours.

(b) Saliva was inoculated with S. coagulatus, and subcultures taken also in saliva; after twenty-four hours biscuit was triturated with the subculture, and tubes filled with the mixture as before and incubated. They both became empty in twelve hours.

(c) Similar experiments were conducted with teeth fixed together with wax in normal positions so as to form interdental spaces (as nearly as possible equal in size). The spaces were then filled with similar mixtures to the above, and the times taken for the spaces to " clear" observed as follows:

Space A (biscuit and saliva), clear in eight days.

Space B (biscuit and saliva, one loop yeast), cleas in fortyfive minutes. 
Space C (biscuit and saliva subculture of S. coagulatus), clear in twenty-one hours.

Space D (biscuit and saliva, trace of S. coagulatus), clear in forty-eight hours.

It is evident, then, from these experiments that the presence of the organism $S$. coagulatus in the saliva very materially increases the rapidity with which carbohydrate material may be broken up and removed from between the teeth. Once being removed, and there being an efficient salivary secretion, it will be swallowed and digested.

Undoubtedly at first a large number of the organisms would also be removed similarly and swallowed; but this, I think, would tend to become less as the organisms became established in the mouth, since, as has already been described, this organism tends to form "plaques," or, as its name implies, to coagulate and to adhere to the glass of the tubes, and it would probably do the same on the teeth in the mouth.

This investigation is not yet complete, but I think sufficient evidence has been brought forward to show that there is every probability of the method being an extremely valuable asset in the preventive treatment of dental caries.

There is, I think, some ground for inferring that some of the native races owe their immunity to the presence of such organisms in the mouth. For instance, it is a custom found amongst certain races for the women with the cleanest mouths to insalivate the material from which it is desired to produce alcohol, in order to start it fermenting. Although the ordinary mouth organisms with sugar do form a trace of alcohol, yet it is merely a trace, and would not be sufficient, I think, under any circumstances to form an intoxicating beverage; and in any case the lactic acid produced would be so much in excess as to render the fluid far more sour than alcoholic. 


\section{CHAPTER XVII}

\section{ALIMENTARY SECRETIONS CONSIDERED AS}

\section{A WHOLE}

\section{Physiological and Pathological Variations.}

CERTAIN suggestions have already been made with regard to the utilization and control of the salivary flow which, if acted upon, would, I believe, very largely eliminate the enormously high incidence of oral disease so universal in civilized communities at the present day.

Now, the suggestions to which I refer are heterodox; they are in complete antithesis to traditional views and teaching. It therefore becomes important to show that such a line of treatment would not be deleterious to the following portions of the digestive canal. It is proposed, accordingly, to show that what is best for the mouth is best for the remainder of the alimentary tract; that, in fact, the several digestive cavities are governed by identical or similar laws; and, further, that, from the point of view of initiating and maintaining normal alimentary secretions and peristalsis, the mouth is the most important part of the whole canal.

Summing up what has previously been said on salivary secretion, we may state:

I. That the salivary secretion is controlled entirely by reflex stimulation derived from the mouth.

2. That the more sapid articles-and of these especially the acid-sweet substances-give rise to the greatest secretion of water, salts, and enzyme; that water is without effect; and that alkalies depress and inhibit secretion. 
3. That the effect of an increased salivary flow is to prevent oral stasis in the mouth, to promote the digestion of starch to a limited extent, to prevent lactic-acid fermentation in the mouth, and to aid in the post-eruptive hardening of enamel (and thus indirectly to prevent the inception of sepsis).

Gastric Secretion.--Here it is proposed to summarize somewhat briefly the work of Pavlov, Starling, Edkins, Lobasov, and others in relation to gastric secretion as observed experimentally in dogs-work which is probably quite well " known of," but perhaps not sufficiently acted upon in practice.

In order to eliminate any stimuli other than those caused by the presence of foods in the stomach, a Pavlov's miniature stomach and a gastrostomy were prepared in the animal; the various foodstuffs were then introduced directly into the stomach without attracting the dog's attention, and the secretion collected from the miniature stomach, which is about one-twelfth of the normal stomach.

Two somewhat surprising results followed: first, the entire absence of secretion to some articles of common daily consumption; and, secondly, the remarkable similarity of response to given stimuli between the gastric and the salivary glands. The following table illustrates the observed effects:

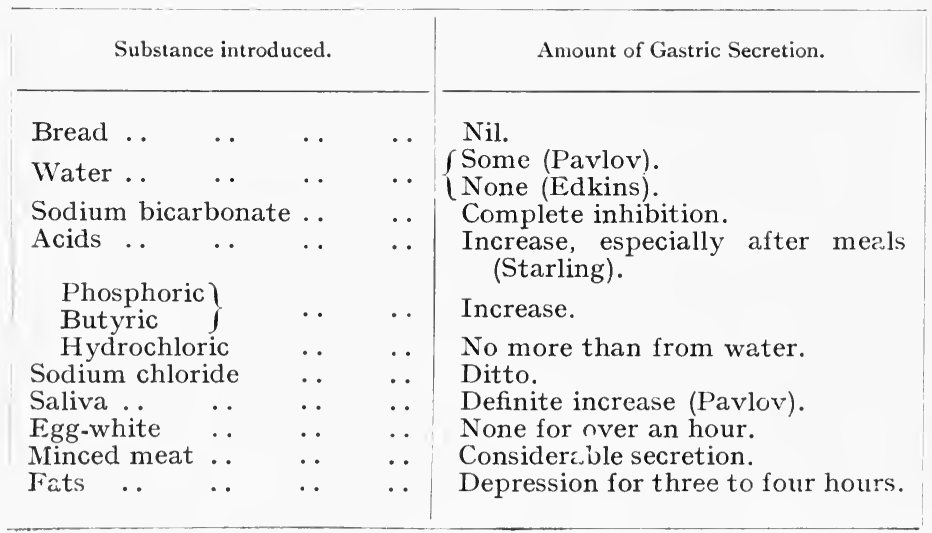


Tested in this manner, we see that, contrary to usual statements, gastric secretion is stimulated by acids and inhibited by alkalies, and that in this respect it is quite analogous to salivary secretion; further, such articles as bread, water, and fats, are depressants of secretion in the second digestive cavity, just as they were found to be in the first.

Pancreatic Secretion, when tested by similar experiments, gives precisely similar results-i.e., the animal has a pancreatic fistula and a gastrostomy, food is introduced directly into the stomach, and the pancreatic secretion observed. The following table illustrates the effects on the secretion produced by various substances:

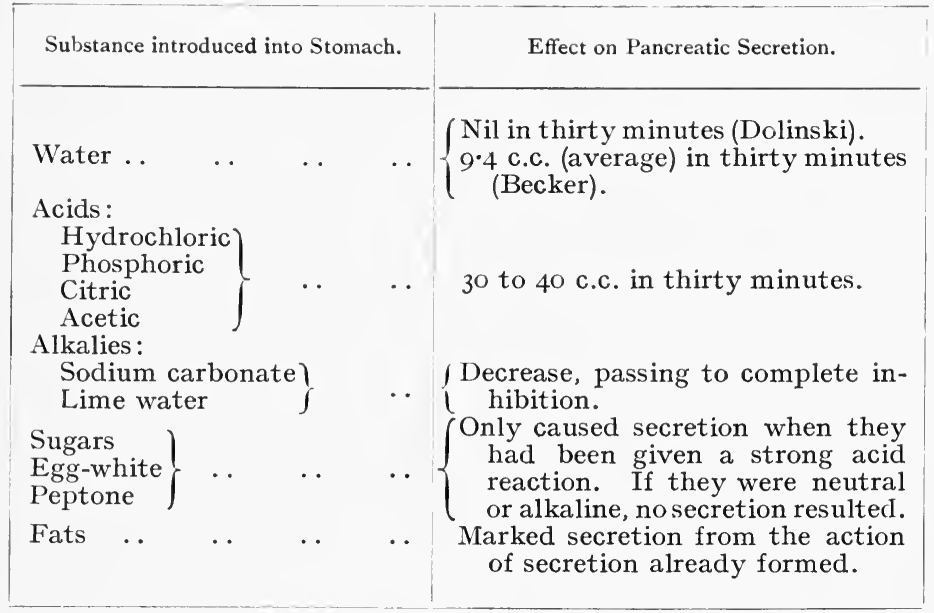

Again the outstanding feature is stimulation by acids and depression by alkalies. It is well known that an acid is necessary to combine with the prosecretin to form the hormone, which is one of the chief stimulants to pancreatic secretions; if, however, foods are taken which stimulate neither oral nor gastric secretion, and which therefore are not acid in themselves, the pancreatic secretion will also fail, for want of hydrochloric acid to form secretion. 
Biliary Secretion by the liver cells is almost analogous to the foregoing, as may be seen in the following table:

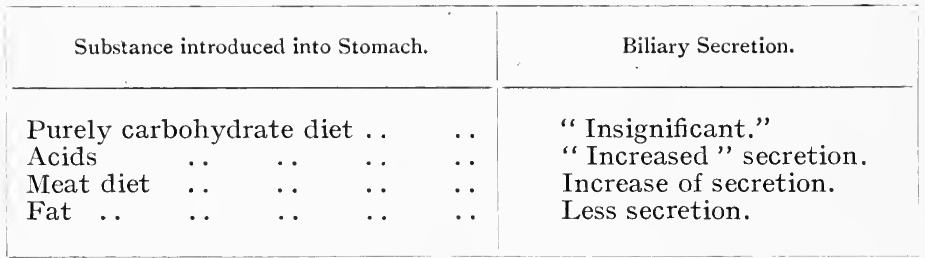

The bile may be secreted, but not discharged from the gallbladder into the duodenum. This discharge has been found to be best promoted by the stimulus derived from the products of active gastric digestion; so that again it is clear that if gastric digestion be feeble (bread, water, alkalies, etc.), the biliary discharge also will be impaired.

We therefore see with regard to these four primary digestive secretions-salivary, gastric, pancreatic, and biliary--that in general terms their stimulants and depressants are identical in nature.

\section{Reflex Digestive Secretions.}

Digestion as a whole, however, is not so simple a matter as it might be inferred to be from the above experimental results, and, indeed, if the above were all the factors concerned, the digestion of many everyday articles of diet would be difficult to initiate and slow of accomplishment.

To a very large extent, gastric, pancreatic, and biliary secretion are dependent, just as is the salivary secretion, upon the integrity and sensitiveness of a reflex arc; and the one half of the arc is, I believe, the same for all.

Pavlov, Lobasov, and others have shown the great importance of "appetite" or "psychic influence" on digestive secretion. Now, although the eyes and ears and nose may to a certain extent act as receptors of stimuli to the digestive glands, yet the nerves of taste must certainly play the major 
part in this rôle; and it is probable that the part played by the other special senses is merely secondary to the education and previous experience of the glosso-pharyngeal and lingual nerves. The efferent part of the path is represented by the vagi, which are secretory to both stomach and pancreas, and motor to the former and the gall-bladder. The accompanying diagram (Fig. 74) represents the paths of action.

If the function of any part of this path be interfered with, there will be a corresponding loss of the alimentary secretions.

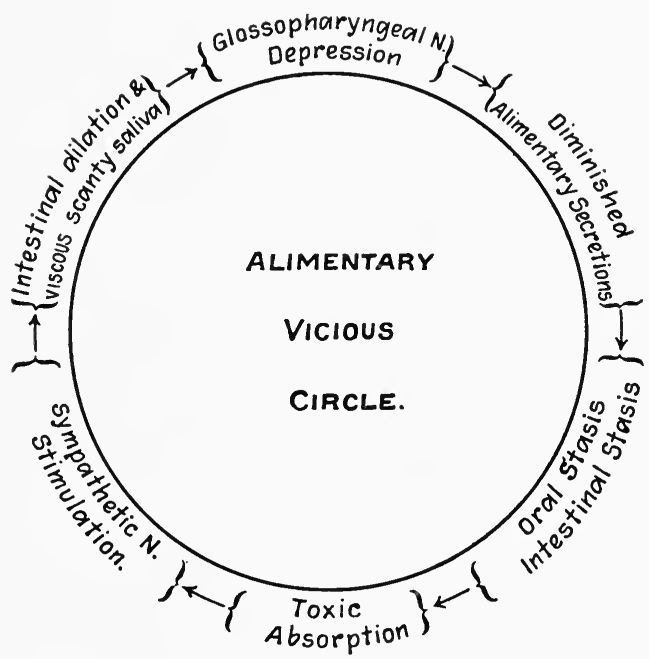

Fig. 74.-Alimentary Vicious Circle.

Reference has already been made to the fact that a merely partial elimination of the terminations of the glosso-pharyngeal and lingual nerves in the tongue by cocaine can be shown to be followed by a marked diminution in salivary secretion. (Experiments have been commenced of a similar nature with respect to gastric secretion.)

Pavlov and Lobasov have both shown that the amount of gastric secretion shows a very considerable increase when the 
food is masticated in a normal manner, and some experiments by Lobasov are particularly eloquent in this respect. Dogs, having been œsophagotomized and gastrostomized, were the subjects of the following experiments:

I. The secretion from a miniature stomach was observed: (a) when flesh was introduced directly into the stomach (i.e., elimination of glosso-pharyngeal reflex); and $(b)$ when the dog eats flesh which passed out at the neck (i.e., glossopharyngeal reflex alone acting).

Gastric Secretion.

\begin{tabular}{|c|c|c|c|c|}
\hline & & & $\begin{array}{l}\text { Flesh introduced } \\
\text { into Stomach. }\end{array}$ & $\begin{array}{l}\text { Sham Feeding } \\
\text { alone. }\end{array}$ \\
\hline Amount in one hour & $\cdots$ & .. & $5^{\circ} \mathrm{O}$ c.c. & $7 \cdot 7$ c.c. \\
\hline Digestive power* $\ldots$ & . & .. & $2.5 \mathrm{~mm}$ & $6 \cdot 4 \mathrm{~mm}$ \\
\hline
\end{tabular}

2. In this experiment a known amount of food was introduced on a string directly into the stomach, and withdrawn after a certain time, when the amount digested could be estimated.

One Hundred Grammes of Flesh introduced DiRECTLy into THE STOMACH.

\begin{tabular}{|c|c|c|c|}
\hline & & $\begin{array}{l}\text { A.-Without Sham } \\
\text { Feeding = no Glosso- } \\
\text { pharyngeal Reflex. }\end{array}$ & $\begin{array}{l}\text { B. - With Sham Feeding } \\
\text { = Glosso-pharyngeal } \\
\text { Aieflex active for } \\
\text { Eight Minutes only. }\end{array}$ \\
\hline $\begin{array}{l}\text { Amount digested: } \\
\text { In two hours } \ldots \\
\text { In five hours }\end{array}$ & $\begin{array}{l}\cdots \\
\cdots\end{array}$ & $\begin{array}{l}6 \cdot 5 \text { per cent. } \\
58 \cdot 0\end{array}$ & $\begin{array}{l}3 \mathrm{I} \cdot 6 \text { per cent. } \\
85^{\circ} \mathrm{O}\end{array}$ \\
\hline
\end{tabular}

From these experiments the considerable advantage to gastric digestion to be derived from an active glosso-pharyngeal reflex-due to the active mastication of a sapid substance —must be quite obvious.

* Estimated by millimetres of egg-white liquefaction in Rett's tubes. 
Reflex Pancreatic Secretion.-Although it has been widely accepted that the pancreas secretes at the bidding of a hormone manufactured in the duodenum and reaching it by the blood-stream, there is undoubtedly an important secretion which is reflex in origin, and which, similarly to the salivary and gastric flow, is dependent upon a glosso-pharyngeal reception.

Pavlov has repeatedly pointed out that the first drop of gastric juice never appears before five minutes after the eating of food in an œsophagotomized animal; therefore there can be no hormone produced under this time. If, however, an animal has a pancreatic fistula, secretion may be seen to start therefrom in from one and a half to two minutes after commencing a "sham meal." Obviously, then, this must be nervous in origin, and the only nerves actually stimulated by the food are those of taste in the mouth.

Wertheimer and Fleig have shown, too, that acid introduced into a loop of intestine with divided bloodvessels produces pancreatic secretion, and the obvious inference is that the stimulus is a nervous one, though it has been suggested that possibly the hormone is absorbed and transmitted via lymphatics which may not have been divided.

Complete blocking of this "oral-abdominal" or "glossopharyngeal vagus" reflex arc may be illustrated by division of the vagi below the diaphragm. When this is done, it is found that the stomach dilates and the food remains in the stomach undigested; it ferments and decomposes there, and the animal dies of sapræmia.

I believe that a similar, though milder-but more prolonged-effect is produced clinically in the human subject by the partial blocking of the afferent side of the arc at its point of origin.

This blocking, or diminished taste perception, is brought about in two ways, which are, however, intimately asso- 
ciated: firstly, by an habitual diet of a non-stimulating character, and, secondly, by the formation of a coating on the surface of the tongue which protects or buries the terminations of the glosso-pharyngeal and lingual nerves, and prevents their being stimulated as they should be by food in the mouth.

\section{Results of a Diet of a Non-Stimulating Character.-From} the foregoing remarks it is obvious that alkalies may be taken as types of non-stimulating, if not of absolutely inhibitory, substances. To test the effect of continual depression of the secretions by this means, I have been feeding rabbits for some three or four years on boiled and neutralized food $\left(\mathrm{Na}_{2} \mathrm{CO}_{3}\right.$ being used). The results are remarkabls, and, so far as I know, have not before been observed. The series of experiments is not complete, and I am unable here to give all the details; it will suffice for the present purpose to summarize the effects, which are as follows:

I. If such feeding be commenced early enough in life, the animal always dies within six months.

2. Development is markedly retarded, the animals only being from one-quarter to one-half the weight of the controls of similar age.

3. Starch and calcium are excreted in excess in the fæces.

4. The salivary glands do not develop in the normal ratio to the rest of the body (lack of taste-reflex).

5. The animals develop a polyneuritis, of which they apparently die.*

6. Post mortem there are practically always gastric lesions, sometimes ulcers, sometimes acute general gastritis, always marked dilatation, and sometimes pyloric stenosis.

The above forms a clinical picture which, I believe, corresponds to the chronic form of a "disease" of which division of the vagi represents the acute form.

* Cooper has recently shown that alkalies destroyed over 50 per cent. of the antineuritic substances which he had isolated from horseflesh, and which were curative for beri-beri in birds. 
These results are, it seems to me, significant in view of the large consumption of alkalies at the present day, both in the form of drugs and in food. There exists, I think, a very serious alkali drug-habit: people dose themselves with bismuth and sodium carbonate almost ad libitum for "indigestion "; the amount of alkali in the form of tooth powders and pastes, partially consumed and used locally to the depression of the oral nerve-endings, must be prodigious; and the amount of sodium carbonate consumed in artificialized foods and drinks must likewise be very considerable indeed.

In some cases alkalies are, of course, necessary, and do a considerable amount of good. They exert this beneficial action by resting overstrained or acutely inflamed digestive organs. In the same way a splint may rest a limb or digitalis the heart, but neither can be continued indefinitely without harm resulting.

Let us now turn back to the second factor in the initial blocking of the normal oral reflexes.

The Presence of a Coating "Fur" upon the Tongue.-Fur is really salivary deposit. It is composed of mucin, epithelial cells, and masses of organisms, and is identical with the deposit found around the necks of teeth. The condition is promoted by a scanty viscous saliva following previous irritation of the mouth by excess of such things as alcohol, tobacco, sugar, or by the over-indulgence in salivary depressants such as tea containing too much tannic acid, bread and butter, and alkalies. But, more important still, it may also be produced, I believe, through the action of the sympathetic nervous system in the following manner:

We have seen that diminished stimulation of the glossopharyngeal chorda-vagus reflexes is followed by a diminution in salivary, gastric, and pancreatic secretions; this means increased stasis, which induces increased proliferation of organisms, with the almost certain production of toxins and 
their absorption. Now, I think there is considerable reason clinically for holding that these toxins-or at least the endotoxins produced-have a definite effect upon the sympathetic or autogenous nerves.

I have tested the effect on rabbits of the injection of endotoxins prepared from human mouth organisms, and I find that they produce a definite, though not great, rise in bloodpressure-probably through vaso-constriction, since the rapidity and intensity of the heart-beat was not increased. This points to sympathetic stimulation. If this be so, then we have the whole train of symptoms associated with severe oral sepsis and alimentary toxæmia explained-the cold extremities, the pale grey complexion, the headache, the constipation (due to intestinal dilatation), the anorexia (due to gastric stasis and fermentation), and the furred tongue; the latter being produced secondarily by the effect of sympathetic stimulation on the salivary glands, which, as is well known, is to cause the flow of a very scanty and viscous saliva of high specific gravity that gets stranded in the papillæ of the tongue and gives the appearance of a dense deposit.

I have also found that by giving rabbits daily, by the mouth, I c.c. of a broth culture from carious teeth, all the symptoms of oral sepsis and intestinal stasis were produced (as far as one could judge in an animal)-at least the symptoms of malaise, anorexia, and diminished defæecation were most marked.

Thus there is set up an extremely strong vicious circle (see Fig. 74). Diminished oral stimulation produces oral stasis and sepsis initially, this in turn producing gastric and intestinal sepsis, thus giving rise to toxins, which being absorbed still further increase oral sepsis and diminish taste-perception by causing intestinal dilatation, which increases toxic absorption. And so the cycle goes on getting stronger and stronger until the patient's alimentary system can no longer resist the strain 
or perform its function; all of which things point eloquently in one direction-the desirability, nay, the necessity, of maintaining the glosso-pharyngeal-chorda-tympani-vagi reflexes in an active and sensitive condition, if the health of the alimentary tract is to be maintained.

How this may best be accomplished is evident from the results of the experiments referred to previously, the chief indications being obviously the more liberal use of organic acids in such strength that they produce a normal and maximum response, and neither fail to stimulate on account of weakness nor paralyze nerve-endings by being too strong. 


\title{
GENERAL CONSIDERATIONS
}

\author{
DIETETIC \\ EDUCATIONAL \\ LEGISLATIVE
}





\section{CHAPTER XVIII}

\section{THE DIET OF RACES RELATIVELY IMMUNE}

\section{TO CARIES}

Although no race may be said to be absolutely immune to dental caries, yet in some races-namely, the Esquimaux and the Maori--it is so extremely rare, compared with the enormously high incidence amongst European races, that a practical immunity may with reason be claimed.

It has been customary for some years now-since the researches of J. R. Mummery-to believe that those races which consumed most meat were the most immune, and vice versa; but, as we shall see, this is on strict inquiry by no means the case.

\section{The Maori Race.}

Instead of discussing vaguely the dietaries of all the various races more or less immune to caries, it will be much more profitable to confine our attention firstly to a detailed investigation of a single race having a high immunity. We shall, too, learn much more of value from a study of the dietetics of a people whose diet was a mixed one, with carbohydrates preponderating, and for whom climatic conditions were similar to those for Europeans. Such a race is the Maori, in whom, as we have seen, the average incidence of caries may be placed at I'2 per cent. In Mummery's investigations the diet is inferred to be largely a protein one, since the Maoris were cannibals; and this seems to be the reason ascribed by Mummery for their comparative freedom from 
caries. Such an assumption, however, is not founded upon facts; the Maori was essentially a mixed feeder, and carbohydrates largely preponderated.

The consumption of human flesh occurred only on more or less rare occasions after a war-party had returned home with prisoners, and then not all the prisoners were eaten; a large number were always reserved as slaves. But, most important of all from our present point of view, the consumption of such food was confined almost entirely to the warriors. Human flesh was strictly tapu-i.e., forbidden-to women,* and only the elder boys were allowed a very small portion. Yet, as a matter of fact, both women and children had just as good teeth as the men. This disposes completely of any theory that the Maoris owed their immunity to caries to cannibalism.

The perfection and also the marked attrition of the teeth of this race have been ascribed by some observers to the mastication of coarse fibrous food, such as fern-roots, mixed with sand. True, they ate fern-root, and also their " ovens" are frequently found among sand-dunes, but neither of these things justifies the above assumption.

The staple food of the Maori was undoubtedly the kumara, or sweet potato (Ipomxa chrysorrhiza); this was cultivated with great care. It was sun-dried, and either eaten at once or stored for winter use.

The rarauhe, fern-root (Pteris aquilina or esculenta), was the next important source of food, but it is very necessary to note the method of preparation. Young roots only were selected, and of these only those having the least fibre. If the root did not break crisply, it was forthwith discarded. The roots were dried and roasted, the outer skin scraped off with a shell; they were then pounded to a powder, and all fibres carefully removed. Water was mixed with it in order to

* Only one woman-the high-priestess-was ever allowed this "privilege." 
make a kind of dough, which was then cooked. Before being eaten it was as a rule flavoured with the juice of the tutu berry, or with the juice of the ti-tree root, the former having an acid taste and the latter a sweetish acid taste.

Taro was another vegetable cultivated by the Maori and largely consumed; it was a perennial, was therefore always eaten fresh, and had a strong and distinctive flavour.

The roots of seedling ti-trees were also eaten after being baked; these contain a sapid sugary juice much valued as a fla vouring agent.

Two kinds of bread were made-one from the pollen of the bulrush, and the other from the berries of the hinau-tree; the latter had a very pleasant taste, and the former was said to taste like gingerbread, and sweet.

Hué, a pumpkin-like fruit, was in the summer season consumed in enormous quantities. A large number of different kinds of berries were also eaten raw; these all had acid sapid properties. The berries of the following trees were the most favourite varieties: the kutukutu (Fuchsia excorticata), the karaka (Corynocarpus lavigata), the poroporo and the poporo (Solanum aviculore and nigrum).

A jelly was also made from a form of edible seaweed called karengo; the latter was first stewed, and then soaked in tutu juice before being eaten.

But it is not to be supposed that the Maori was a vegetarian: far from it. Yet, since there were practically no mammals indigenous to New Zealand,* his animal food consisted entirely of fish and birds, to obtain which he went to considerable trouble. The difficulty, however, in obtaining such food must have been so great in many cases as to preclude the possibility of its forming anything but a subsidiary part of

* A very small rat and a dog were indigenous, at least as far back as legend and history go; both these animals were eaten, but the latter was tapu as an ordinary article of diet. 
his dietary. For instance, the Maori used no projectiles of any sort, and all his birds were either snared with a loop on a long pole or speared with a long light spear. In this way were obtained the following: kukupa (pigeon), kaka (parrot), putangitangi (paradise duck), pukeko (swamp-hen), kiwi (apteryx), weka (wood-hen), koreké (quail). Also a large number of young sea-birds, including " mutton " birds, were collected in season, and dried for winter use. The birds, however, it will be noticed, were all rather small, and, with the exception of the putangi, several would be required to make a meal for a family.

As regards fish, the Maori used small nets only in rivers and estuaries, and a line and hook in salt water. The hook was not "baited" as we understand it, but simply had a piece of iridescent paua-shell attached; and thus were caught hapuka (cod), barracouta, moki, patiki (flat fish), and also snapper, kingfish, and butter-fish. Sharks were occasionally caught, and were esteemed a great delicacy. The inland tribes depended upon fresh-water fish, of which eels were the chief variety. These were trapped as a rule in weirs constructed of reeds. Piharau (lampreys) were caught similarly. By means of small seine nets were also caught ınangi (a kind of whitebait) and upokororo (a kind of grayling).

Crayfish and shellfish also formed a part of their regular dietary, especially the paua (a large clam); these were collected, sun-dried, and stored, and also sent inland to other tribes in return for " potted " birds.

Cooking.-Most of the animal food was cooked together with " vegetables," such as kumara, taro, raruaké, by being steamed in an earth oven for about an hour and a half. Some of the larger birds were roasted on a spit. One of the chief aims in the process of cooking was to keep all the " flavour" in by wrapping the food in layers of green leaves, and covering the oven with mats and earth. The leaves 
having once been opened, the food was either eaten or thrown away; no food was ever recooked or touched a second time upon any consideration whatever.

The Maori was allowed to exercise his idiosyncrasies of taste, and was not bound by any custom or law to eat what he did not relish; he simply stated that he was "wainamu " for certain food-even human flesh in many instances-and that wish or declaration was accepted by parent, host, or guest, without demur.

As a rule two meals a day were taken, at ten and four o'clock; in times of scarcity this number was reduced by half. As regards drinking, the Maori drank only plain water, or water flavoured with the juice of the tutu berry.

Masticatories were largely indulged in. The gums of the kauri and tarata trees were freely chewed, as also was bitumen, these being passed unceremoniously from one mouth to another. I have been unable to gather any evidence either from the Maoris themselves or from other observers that they ever practised cleaning the teeth by any artificial means.

\section{Summary.}

Thus it is obvious-

I. That the dietary was essentially a very varied one.

2. That the Maori could not possibly have owed his immunity to a preponderance of animal food.

3. That the methods of preparation of vegetable and carbohydrate food generally was such as to eliminate all the obviously coarse and fibrous constituents; nevertheless, since the methods of preparation were of necessity crude, a certain amount of the finer particles of cellulose must have remained in all their food, even in the bread. That nearly all food was steamed, thus considerably reducing its toughness.

4. That a considerable amount of fruit and berries was consumed. 
5. That throughout the whole dietary one of the most important factors was taste and flavour.

(The character of the food, both protein and carbohydrate, was such as had in itself distinctive flavours-namely, fish and game, sweetpotato and fern-root, berries and fruit.) That, in addition to this, substances, such as the tutu and ti-tree juices, were constantly used to increase the sapidity of the ordinary food.

6. The use of the tutu-berry juice is particularly interesting. There is no doubt that it acted as a stimulant of alkaline saliva by reason of its acid-sweet flavour; but it also in all probability had a further influence on the salivary secretion through the action of its active principle, tutin, on the nervous system. Fitchett* found that after hypodermic injection of tutin in the cat, among the first symptoms in five cases out of six was intense salivation.

Now, although there should be very little tutin in the juice of the berry, I think that undoubtedly some is very frequently adventitiously present, judging from the cases in which toxic symptoms have arisen in Europeans after sucking the berries or drinking wine made from the berries when great care had been taken to exclude the seeds. Fitchett also showed that tutin was not excreted at all rapidly. It would therefore appear to be not at all improbable that the Maori, by the constant ingestion of even minute quantities of tutin, kept the efferent salivary nerves in a state of excitability, if not of mild excitation.

7. The use of masticatories also undoubtedly kept the salivary glands in a state of activity, and both their secretory powers and the total daily amount of secretion would be thereby greatly increased.

Moreover, the habit of chewing prevented "stagnation" within the oral cavity, and so removed one of the great predisposing factors to disease.

* "Physiological Action of Tutin," by F. Fitchett, M.D., Transacions of the New Zealand Institute, I908. 


\section{Conclusions.}

It is therefore clear that the relative immunity of the Maori was due, not to an excessive protein diet nor to a great excess of hard, fibrous, inert matter in their carbohydrates, but to the habitual and constant mastication of salivary stimulants, thus producing a constant flow of alkaline and diastatic saliva, the beneficial effect of which has been previously considered.

\section{Other Races.}

It will be found upon close investigation that similar dietetic principles to the above pervade almost every "natural" race; and although it is not possible here to consider in similar detail the foods of all other races having a comparative immunity to caries, yet a brief consideration of the various dietaries is important.

Esquimanx.-It is quite usual to suppose that the Esquimaux exist entirely upon animal flesh, fat, fish, and birds. A little thought, however, will show that this cannot be so; no one can be entirely deprived of all fresh carbohydrate food without ill results, and, indeed, investigation shows that the Esquimaux themselves recognize this. Kjellmann says that no less than twenty different kinds of plants are used for food, and that "provisions of berries and of reindeer moss rich in starch are stored up for the winter." The berries are a kind of small cranberry, the acid-sweet taste of which is well known. They also brew a fermented liquor of a sourish quality. (Incidence of caries, I*4 per cent.)

Among the Lapps it is customary to supplement the milk with sorrel or butterwort, and Ratzel affirms that " similar acid or pungent plants are welcome to all polar people."

The inland Arctic people of North Asia live largely on bilberries, supplemented by the products of hunting and fishing. 
The Samoyedes, a nomad Arctic people, unlike the Esquimaux, have numerous wives, whose duty it is, whilst the men fish, to seek for shells, herbs, and berries.

The Indians of the North-West American Coast are very Esquimaux-like in their diet, subsisting largely on fish, supplemented by roots, grasses, and berries; and a cultivated potato-patch is usually attached to each " house." All food is cooked by the steaming process. As a masticatory the wood of the sugar-pine is chewed, which travellers assert tastes as much of turpentine* as of sugar. (Incidence of caries, 3.9 per cent.)

The Fuegians, inhabiting the other extremity of the American coast, are also said by some travellers to exist chiefiy on fish partially cooked, to which are ascribed their broad zygomatic arches and powerful masticatory muscles. Darwin, however, whose accuracy as an observer cannot be doubted, says that (at least when he visited Tierra del Fuego in 1834) their staple food was a dried and hardened fungus (Cyttaria Darwinii), which has "a mucilaginous, slightly sweet taste, with a faint smell like that of a mushroom."

Oceanians, Polynesians, and Melanesians. - Here again it is popularly imagined, because many of the inhabitants of the Pacific Islands were (and some still are) cannibals, that their diet was essentially a protein one. On the contrary, as with the Maori, human flesh was consumed only on very special occasions, as after a raid on a neighbouring island or at the opening of a "tambu " house or other high festival. Those who have lived in the islands are all unanimous that by far the larger proportion of food in all cases consists of vegetables and fruit.

The Fijians, perhaps, eat slightly more meat than other islanders. Their animal food consists of fish, opossums, and

* The important pharmacological characteristic of turpentine in this respect being that in the mouth it has a pungent, bitter taste, and causes a reflex flow of saliva. 
less frequently pigs. All meat is cooked, usually steamed until very tender; the opossum is said to have a "strong flavour," and their roasted pig is thought by some to be superior in flavour to English bacon.* Meat is frequently eaten in a partly putrefied condition. But even the Fijians' food is principally vegetable, of which the following are the varieties chiefly consumed: yams, taro, sweet root of ti-tree, banana, sugar-cane, and cocoanuts.

Most of these vegetables and fruits are common to all the islanders, some kinds being more cultivated in particular islands than others. Poi, a kind of sour porridge, is made by most Polynesians from taro flour, by allowing it to ferment. Sago is largely eaten by some tribes; it is most carefully prepared and cooked, the coarse fibres being removed first of all by an ingenious system of washing.

In some parts of Polynesia (particularly in Tahiti) breadfruit is fermented into a sour paste and set aside to store, since in this condition it keeps well.

"Dessert," as already mentioned, takes the form of puddings made by first roasting taro or bread-fruit, then pounding it into a stiff paste and flavouring with fresh cocoanut dressing. "Puddings are practically swallowed without chewing." "On the whole a native repast is not masticated so much as an English meal, because there is less to chew, the staple being vegetable." $\dagger$

Fruits, of course, form a large and important part of these islanders' dietary. In addition to those already mentioned are the mango, a large pumpkin, a variety of cucumber, a species of lime, the kernels of the borolong and saori, the fruit and leaves of the asi-tree (these having a decidedly acid taste), and many others.

Many Polynesians season their dishes with salt sea-water. (Incidence of caries, $5 * 2$ to I9 per cent.)

* Guppy: "Solomon Islanders," I 883.

† W. Deane, M.A.: "Daviu Levu." 
Kava,* a beverage of bitter (or sour) flavour, is largely consumed in a great number of the islands. It is prepared from the roots of the Piper methysticum; the roots are chewed by women and girls, then expectorated into a bowl or trough, and water is added (this method, however, is not universal).

New Hebrides (Mota).-The staple food, as stated on p. 47, is cooked yam, of which there are about forty varieties. Fish, shellfish, crayfish, and crabs, are favourite accompaniments. Pig is reserved for feasts. Yam and taro are frequently made into mashes called loko and lot respectively. Bread-fruit is dried and eaten crisp or soaked in a mixture of young cocoanut juice and sea-water. This salty-acid flavouring is extensively used on all dishes - the native green " toape " and many other articles being cooked till soft and also thus flavoured.

The banana, wild pandanus, pineapple, orange, mummy apple, rose apple, quava, spondias dulcis, are all eaten raw. Nuts of many kinds-the principal, of course, being cocoanut -are extensively eaten both raw and cooked.

The Chinwans of Formosa brew a fermented drink from rice and millet, yeast being replaced by the saliva of an old woman in a similar manner to its use for kava. A like custom is observed among the Indians of Central America in Guiana, where an intoxicating beverage called "paiwari" is made in a similar manner from cassava bread, the fruit of palms, maize, and bananas. In this case the mass is sterilized, by pouring on boiling water, before being chewed by the women. It is said to be a "cooling" drink somewhat resembling sour beer.

The Papuans have as their staple food the kumara and sago, diversified by large broad-beans and the leaves of the tree-cabbage. A kind of palm is cut down when they are hungry, and the inside leaves of the top green shoot are

* I find that the authorities I had relied on, when in the first edition I stated that kava was alcoholic, are wrong. Further enquiry has shown that it could not have been fermented in the old days, and certainly is not now. 
eaten; these are "straw-coloured and like asparagus, sweet and slightly dashed with acid "; of this the natives eat great quantities. Bread-fruit is eaten boiled; this is said to be palatable, though slightly bitter. The Papuan, however, is extremely partial to animal food, but it is necessary to observe the conditions under which it is eaten.

The pig, cassowary, and wallaby are hunted by being driven into corrals made of nets set in the forest. The game is roasted, and in an incredibly short space of time every particle is eaten, and the people then lie about in a gorged condition. Such "drives" occur only at long intervals. Papuans are also very fond of a large tropical beetle (one of the Passalidæ) found in decaying tree-trunks; it is usually eaten raw, but may be just warmed in the fire; the flavour resembles that of a lemon.

In the islands, yams and taro form the staple diet; to these are added bananas, sugar-cane, nuts, fruits, berries, mangoes, bread-fruit, and cocoanuts. Animal food is rare, and fish is scarce, since the waters are deep.

Betel is extensively chewed; but I was informed by a native chief from one of the islands that this does not produce any discoloration of the teeth, the latter being brought about purposely for æsthetic reasons by the use of a certain " earth."

As a beverage the "soup" water in which fish or yams have been boiled is drunk. Fermented liquors are unknown.

Malays.-The staple food of the wilder tribes of the Malay Peninsula consists chiefly of such wild yams, roots, and fruits of the jungle as may be in season. When this fails, the men engage in hunting, trapping, and fishing. In other more agricultural tribes, rice, maize, millet, tapioca, and bananas, form the staple articles of diet.

The Semang tribes exist on small birds and animals, various kinds of yams and roots, with the addition of gourds, pumpkins, chillies, maize, and sweet potatoes. 
The Sakai live upon wild tubers, roots, and fruits, and some animal food; they do not, as a rule, search for game until everything else fails. One kind of apple-like fruit eaten is said to be very pleasant, but to leave a most decided "after-taste."

Jakun peoples, like the two former tribes, are markedly frugivorous. Logan gives lists of forty and sixty-nine different kinds of fruits which various Jakun tribes are in the habit of eating. The latter tribes eat more rice and less meat than the two former, but the rice is always flavoured. Skeat says: "I have myself frequently seen the latter at their meals, when their only food consisted of boiled rice seasoned with acid fruits obtained from the jungle." Other seasonings used are kulim-leaves (resembling onions), wild ginger, turmeric, species of eugenia, wild pepper, and various spices. When the people disperse, as they do each year, for long periods through the jungle in search of fruits, they live entirely upon tapioca root and fruit. Favre, however, speaking of these tribes, says that their diet is very varied, and nothing can be said to be regular, and when opportunity arises they will eat the flesh of any available animal. Honeycomb is greatly prized, but it is not eaten until the small bees are well formed in the cells!

The tribes on the seaboard exist on rice principally, to which is added the flesh of the hog, monkey, snake, and birds, together with vegetables and wild fruits. All these tribes have a great desire for the durian fruit, some living upon it entirely for six and eight weeks, and in one case Thomson found six boat-loads of Sea-Jakun, who had travelled I8o miles by sea to obtain this particular fruit.

The Indians of North AND Central AmericA.-It is stated by Mummery that the diet of the North American is mainly meat; with this Ratzel does not agree. Although this authority admits that animal food is eaten with avidity, 
yet he states that the chief article of diet is still the maize, and that large quantities of fruits, berries, and nuts, are collected, and also stored for winter use. Wild melons, plums, and grapes, are recognized food items for tribes in the interior.

Greatly esteemed is the large bulb of the Lilium columbianum and the bulbs of other lilies; in addition, the roots of the sunflower, white clover and bracken, wild onions, and wild carrots, are largely eaten.

A great variety of berries are gathered and eaten, both fresh and preserved, the most favoured being the "Service" berry-a kind of black currant (Amelanchier alnifolia); in addition, the following are also eaten: red and black currants, gooseberries, blackberries, strawberries, raspberries, whortleberries, soapberries, cranberries, crowberries, cho keberries, elderberries, bearberries, jumperberries, roseberries, mooseberries, and salalberries. (The latter is a species of Gaultheria )

The food of all New Mexicans (Indians) is similar. They make pretensions to agriculture, and are used to a vegetable diet; they seldom, however, raise sufficient to last until the new crops are available, and so have to subsist on "beans, nuts, and other wild fruits, which they collect in considerable quantities." Of animal food, comparatively small quantities of rats, lizards, squirrels, and fish, are eaten.

In Central America also the Indians much prefer meat, but "totemism" frequently prohibits many animals. In addition, the quinoa and the potato are the chief natural food of these races; manioc and cassava are also used; and in Chili it is said that the straxeberry, which covers miles of country with its runners, is an important article of food. The fruit of the carnahuba and mariba palms are, too, favourite articles of diet; these have an agreeable, juicy, sweet flesh, and are utilized in various ways. 
Wild tribes of Central America, according to Bancroft, are essentially agricultural, living on maize, beans, bananas, and plantains, together with a variety of fruits and roots. Fish, eggs, turtles, and fowls, with the inevitable chilli (and salt) for flavouring, compose their animal dietary.

The " Natural " Races of AfRica.-The diet of the negro in general may be said to be both animal and vegetable, with wide variations for the various races, some races consuming a minimum of carbohydrates, and others a minimum of flesh protein. Nearly all races, except the Bushmen, Dwarfs, Masai, and Galles, cultivate the following crops extensively: millet, maize, manioc, and banana (Equatorial). Coffee, guru, and kola nuts are habitually chewed by many races; salt (sodium chloride) is in great demand as a seasoning by all, and when this cannot be obtained potassium salts obtained by evaporation of lake water are substituted. The practices of smoking Cannabis indica and of brewing beer by the fermentation of maize or millet are found in very many races.

Some races clean the teeth and scrape the tongue artificially with a frayed-out stick dipped in ashes, and spend a considerable time each day on the process.

The natives of British Central Africa eat " surprising quantities" of a stodgy kind of porridge made from maize or millet flour, but do not at all like it without salt or some form of ndiwo (relish). Sweet potatoes and manioc root (raw and cooked) are also eaten; and in the spring pumpkins, gourds, and cucumbers, are principal articles of diet; but comparatively small quantities of meat are eaten, and then it consists chiefly of rats, very high fish, locusts, white ants, and occasionally fowl or goat's flesh.

In Uganda the natives in many parts (Waganda) have advanced somewhat higher in the scale of civilization and its arts; they are " mixed feeders," living on the flesh of domesti- 
cated animals, such as sheep, goats, and fowls, together with bananas, maize, sweet potatoes, yams, and various fruits.

The Manganga (around Lake Nyassa) eat vegetables chiefly; they consume neither eggs, milk, nor poultry. They catch and dry great quantities of fish, which, together with salt, they trade to other tribes in the interior at great distances.

The Kaffirs and Hereros are essentially cattle-breeders, but are extremely reluctant to slaughter animals merely for their own food; at feasts, however, and on special occasions they will consume enormous quantities of meat.

The usual morning and evening meals of the ordinary Kaffir consist of a porridge and amasa (sour milk), one or other vegetable, millet "beer," and occasionally some meat. (Incidence of caries, $14 \cdot 2$ per cent.)

The Negroes of West Africa also live on grain, chiefly supplemented by aromatic herbs and much salt. Cayenne pepper is very popular.

The Bushmen are a fierce nomad people living chiefly on the product of the chase, supplemented by edible roots, honey - a very favourite and frequent article of diet-and a wild water-melon, juicy and bitter in flavour. They cover great distances in their marches, and suffer considerable privations, and the water-melon is said to be frequently their only possible drink. (Incidence of caries, 20.6 per cent.)

The Pygmies of Central Africa are likewise a nomadic tribe, living chiefly on the products of the chase, some of which they exchange with adjoining agricultural tribes for plantains, potatoes, etc.; their own carbohydrate supply consists of fruit, nuts, and honey.

The Abyssinians are mixed feeders, and are more highly civilized than other African races (except the Egyptians). In making their bread (teff), they always include a sauce of red pepper to render it sapid.

The Kabyles of North Africa live chiefly on onions, cucum- 
bers, gourds, and water-melons, and they are especially fond also of a small wild artichoke.

The Moors of Morocco are people of very ancient civilization, but nevertheless their mode of life is considerably simpler than ours, especially among the poorer classes. Kesk'soo, prepared from semolina, is the national dish; zumetah (parched flour) and vermicelli are also used for making cakes; very hard doughnuts, flavoured with anise and caraway seeds, and called " fikaks," are commonly eaten. In addition, rancid butter, honey, dates, raisins, and large quantities of ripe melons and black figs, form an important part of the food. "Kabab," made from chopped-up and seasoned meat, partially cooked, is a very favourite dish; salt is used extensively. Various kinds of confectionery are largely eaten, so that it is not surprising to know that the native "doctors" are acquainted with, and use three different instruments for, the extraction of teeth-namely, a native instrument which is " a sort of exaggerated corkscrew without a point," and also keys of French and English pattern. The possible presence of diseased teeth and the value set upon good teeth are shown by the fact of slave-dealers always examining the teeth and gums of slaves before purchasing.

Australian and Tasmanian Aborigines.-Both of these races were nomadic, and lived largely on flesh food, such as insects, fish, birds, reptiles, and very many marsupials. Various roots, fruit, honey, and the gums of certain trees, were also articles of diet. (Incidence of caries-Australians, 20.5 per cent.; Tasmanians, 27 per cent.)

Probably altogether they employed some three hundred different kinds of vegetable or fruit food, but no crops were cultivated in the ordinary sense of the word. Yams, nardoo, and bulrush-root were staple foods in many districts. The former two were made into cakes, and the latter is said to have occupied the position that bread does to the European; 
but it was very coarse, and the fibrous parts were spat out after chewing. Meat was frequently flavoured with the sweet secretion from a species of Psylla.

A fermented liquor was prepared from the fruit of the pandanus. There are two things, however, which operated adversely in the diet of the Australian: firstly, his practice of eating clay after meals, and of mixing earth with yams before eating them; and, secondly, his frequent difficulty in obtaining water, owing to periods of drought, or to his nomadic habit and the consequent necessity of passing over arid areas. The Tasmanians were not cannibals, but the Australians were; and in many parts human flesh was consumed by both sexes and all classes, and sometimes one child of a family would be killed and given to another child to eat in order to strengthen it.

The Inhabitants of IndiA.-The races, tribes, and castes, of India are so numerous that it is impossible to give any concise and accurate description of a diet which will apply to all. Rice is the staple food near the coast, millet in the Central Provinces, and wheat in the Punjab and North-West. In all cases the cereal is served in a variety of ways, usually retains some of the husk, and is seasoned with herbs or spices, the Indians being, of course, extremely fond of the latter. Eggs, milk, fruit (ginger, plantains, citrons, gourds, pumpkins, and in some parts oranges and lemons), and salt, also form a regular part of meals in most instances. In some parts -e.g., Khondistan_- "butter" is made from two varieties of sinapis. Fish is abundantly eaten on the coast, but not in the interior, the art of angling not being usual.

Among many of the tribes of Northern India, the women collect considerable amounts of wild vegetable food with " digging spuds." Poisonous tubers are soaked in water to render them innocuous. The fruit of the Mohwa furnishes the food of the poorer classes for several months, and is usually 
eaten with sal seeds or the leaves of various jungle plants, together with a little rice. The seeds of many wild grasses and bamboos, and the leaves of leguminous plants, form, incieed, a very important part of their food.

The Todas, in Southern India, exist at the present time chiefly on milk, butter-milk, ghi grain, rice, and sugar; but from their own statements they formerly lived on roots, herbs, honey, and fruit. The flesh of the calf is eaten only three times a year, but that of the sambhar may be partaken of at any time when an animal happens to be killed. During pregnancy the women drink the juice of the tamarind, which is markedly acid, but pleasant.

JAPANESE.-The principal food is rice, also buckwheat, wheat, barley, taro, and yams. A seasoning of some sort is used with every meal, usually either a white radish or the fruit of the egg-plant. The fruits of the kaki and biwa are largely eaten, as also are cucumbers, water-melons, and parts of the chrysanthemum flower. Meat is a rare delicacy.

CHINEse.-The staple food of all Chinese is rice; it is served in a variety of forms, but is to European taste always " underdone." In addition, taro and sweet potato are largely eaten, and, of course, the Chinaman is proverbially a past-master in the art of cultivating "vegetables," which form an important part of the national menu. The Chinese are almost vegetarians by force of circumstances, and not so much by choice. Fish, salt and fresh, duck, and pig, are the chief articles of flesh food, and in this order of frequency, pig being consumed by the middle-class and poorer Chinese on festive occasions only. A little mutton and beef is eaten in Northern China. Fruit is abundant in most parts of China, especially the south, but near the large towns and ports it is sold for export rather than eaten.

Missionaries are of the opinion that the Chinese, in the country districts at least, have "most excellent teeth." 
Persians.-There are three national dishes: chillau (boiled rice with a little grease), pillau (boiled rice with lamb), and ash (rice stewed with vegetables and fruit). Sherbet, the national drink, is made from iced water and fruit juices and essences.

\section{General Remarks and Conclusions.}

The incidence of caries in these races varies considerablyaccording to Mummery's figures, from $\mathrm{I} \cdot 4$ to 20.8 per cent. (of individuals); and according to Patrick, from 2.064 to 5.804 per cent. (of the total number of teeth). The latter figures are really the more important, since they give a more correct estimation of the extent of caries in any race. Now, as we have seen (pp. 9, I0), even the highest of these figures is incomparably lower than those obtaining amongst civilized races at the present time. So that we may group all the "natural" races together, and endeavour to ascertain whether there is anything common to their several dietaries which would explain their relative immunity to caries. Mummery and Miller believed that a larger consumption of meat explained the difference. This theory, however, although a factor, must per se fall to the ground, since both Mummery's and Patrick's figures (see pp. 9-I2) show that several meateating races have a higher incidence of caries than non-meateating. Also it is of the very highest significance that in Patrick's list the great rice-eating, almost vegetarian races of South-Eastern Asia should show the least incidence of caries; out of 2,000 teeth, only 2 per cent. were carious.

Again, many of the people showing the highest incidence of caries (the colonials of Australia and New Zealand) consume enormous quantities of meat-namely, 240 and $25^{\circ}$ pounds per head per annum respectively-yet the number of individuals affected by dental caries is estimated to be from 90 to 95 per cent. (by the Australian and New Zealand Dental Associations). 
Therefore it is obvious that upon these considerations alone a large consumption of meat does not confer even a relative immunity.

From this investigation several things stand out clearly: That the diet of all "natural" races and of those relatively immune to caries is essentially a varied one, and that although certain articles form the staple food over certain large areas, yet they in no case form the sole food of any race.

There are, then, two general principles to be found running through the dietaries of all the natural races. They are-firstly, variety, and, secondly, sapidity.

This enquiry was undertaken quite impartially, and it was not anticipated that the results would so amply bear out the previous investigations. The same conclusions are made by Ratzel, an eminent authority on ethnology, but who had no reason to pay any particular attention to any one feature of the foods of such races; yet, summing up the characteristics of the partially "cultured " people (Chinese, Japanese, Hindoos, Arabs), he says: "Variety of food is great, and the sense of taste appeals at a very early stage."

The constant inclusion of articles in the food which have a direct stimulating effect upon the salivary glands is the one and only link which connects up all the races showing a relative immunity to caries. It is common alike to dwellers in the Arctic and the Equatorial regions, it is found in meateating and in vegetarian tribes, it is present in both low and high types of uncivilized man.

Further, it will have been noticed that although a variety of sapid substances are used, yet the ones most frequently recurring have an acid reaction-chiefly fruits and berries; and, as has been previously shown, these are the very stimulants which produce the most profuse and the most alkaline flow of saliva, and to this latter fact I think we are justified in ascribing the relative immunity found in the races which have 
been considered. Only on such a basis can the order of incidence in Patrick's figures be explained.

In Southern Asiatics the percentage is least, and these are the people who have fruit in abundance, and use it as an ordinary article of food, and not merely as a luxury at their meals. The Egyptians and Africans come next, including races which employ both fruit and salt largely (and also artificial prophylaxis). Polynesians and Australians are next, the percentage doubtless being raised considerably by the inclusion of the nomadic Australians.

Central Americans follow, whose salivary stimulants are mostly sweet-acid, but whose comparatively higher incidence may be due to their consumption of cassava, which is " dry tasteless stuff like sawdust."

North Americans and Esquimaux are grouped next, although these people are amongst the largest meat-consumers. They both eat fruit and berries (and the Indians sugar-pine), but the amount must of necessity, owing to climatic conditions, be comparatively smaller.

The highest incidence of carious teeth falls to the South Americans, including the Guachos, whose diet is mainly meat, and the Fuegians, who consume fish and fungus largely. The relatively higher incidence is also possibly caused by the inclusion of many earth-eating tribes, by the consumption of cassava bread, and by the general laziness of the Indians, who strongly " object" to cultivation. This, also, is one of the very few countries where salt was not used at all by the natives to increase the sapidity of their food.

Totemism and TABOos.-In probably the greater number of the natural races of mankind, food restrictions of one kind or another are enforced. In many cases this is due to totemism. An individual has a certain object for his "totem"; this is always some well-known animal, plant, or natural feature of the landscape. When the totem is an 
animal or plant, it becomes prohibited as an article of food. (There are a few abnormal exceptions to this rule, but they are unimportant.) The interesting feature from the present point of view is that the great majority of totems appear to be animals, and this, therefore, would tend to restrict the consumption of animal food. The fact, too, that the totems of individuals of a tribe or family are not the same-in fact, in many cases are necessarily diverse-would further tend to restrict animal food. For instance, there might be six different animal totems in a family of seven or nine, so that whichever of those six animals happened to be for dinner, certain members of the family would be unable to partake of it. Other food restrictions are related to sacred rites and seasons, or are enforced at the period of puberty or afterwards for supposed sexual reasons. In these cases, also, the prohibitions relate chiefly to animal food. For instance, in certain Australian tribes there are thirteen different kinds of animal food which a boy may not eat before puberty, and eleven kinds which a girl may not eat until she becomes a mother. Amongst certain Melanesian tribes no man until past middle age is allowed to eat dog, turtle, or six particular species of fish, and no woman may ever eat the two former; whilst in some places only middle-aged men may eat pig. These latter prohibitions are enjoined because the consumption of such animals is supposed to create a dislike by the opposite sex. In many instances animal food is taboo to one or both parents for a variable period after a child is born; in the case of the Jakun, until the little one has acquired the use of its legs.

In various countries, too, where cannibalism was practised human flesh was generally taboo for women, and not infrequently also that of certain animals, such as the dog.

Attrition of Teeth in Native Races.-Nearly all races which are comparatively immune to caries show marked attrition 
of the teeth. It has been assumed that this must have been due to an excess of coarse or gritty material in the food; but, as we have seen, this cannot be held to have been anything like sufficient in amount, since nearly all native races are most careful, in the preparation of their food, to eliminate all the obviously coarse particles and to cook it thoroughly. It is far more probable that the attrition is due to the mechanical effect of finer fibrous particles, combined with the constant action of the acids of fruits and berries. In fact, this "attrition" is to be regarded as in every way analogous to the "wasting" produced experimentally by Miller upon the labial surfaces of teeth by the combined action of acids and tooth-brush, and simulating erosion cavities. A similar condition of attrition or "wasting " of the occlusal surfaces of the teeth is seen in people of gouty diathesis, and, as is well known, a majority of such people are bons vivants, and as a rule have a preference for acid beverages (such as port wine); but it cannot be claimed that their dietary is gritty, or coarser than that of members of the same household who do not suffer from gout or attrition of the teeth. Undoubtedly, also, the musculature is more vigorous in native races and gouty individuals, and the "force" of mastication therefore greater; thus the acid and fine fibrous particles are aided in wearing down the cusps of the teeth.

\section{BIBLIOGRAPHY OF WORKS WHICH HAVE BEEN CONSULTED.}

Bancroft: Native Races of the Pacific States of North America.

Brown, J. M.: Maori and Polynesian.

Crooke: Natives of Northern India.

GUPPY: The Solomon Islanders.

HADDon: Head Hunters, Black, White, and Brown.

Hill-Tout: British North America.

Howitr, A. W.: Native Tribes of South-East Australia.

Lloyd, A. B.: In Dwarf Land and Cannibal Country.

MEAKIN: Life in Morocco.

Pratr: Two Years among New Guinea Cannibals .

Ratzel: The History of Mankind. 
RIVERS: The Todas.

Seligmann: The Melanesians of New Guinea.

Skeat and Blagden: Pagan Races of the Malay Peninsula.

Spencer and Gillen: Native Races of Central Australia.

TAYLOR: Early History of Mankind.

Thomas, N. W.: Natives of Australia.

Tregear: The Maori Race.

Werner, A.: The Natives of British Central Africa.

Various Communications to the New Zealand Institute. 


\section{CHAPTER XIX}

\section{THE DIETARIES OF MODERN CIVILIZATION : THEIR ERRORS AND CORRECTION}

Attention has been directed earlier to the general "softness" which characterizes modern diets, resulting in a lack of sufficient stimulus to the jaw-bones-therefore in a crowded and irregular state of the teeth, and hence a predisposition to caries. The elimination of all fibrous elements from food, too, probably tends, as Wallace* has pointed out, to the increased lodgeability of foodstuffs, by robbing them of their detergent action upon the teeth. But, as I have shown, the inclusion of a large element of cellulose in foodstuffs does not of necessity prevent them from lodging and forming an appreciable amount of acid.

It does in some cases reduce the acid-production, but the reduction bears no relationship to the amount of cellulose present.

There is another characteristic, however, of our modern dietaries which has been overlooked. I refer to their comparative non-sapidity, their usual neutral or alkaline reaction, and their general uniformity, all of which are properties which have been shown to be incapable of reflexly exciting a suffcient secretion of saliva to keep the mouth free by natural means from fermentation.

One only has to reflect for a short time, to come to the conclusion that the substances of which we consume most are those which have the least taste. In fact, para-

* "The Physiology of Mastication," London, I903. 
doxical as it may seem, modern communities have acquired or developed a " taste" for tasteless things. This " taste" is not evolved; it is, I believe, acquired by a process of training and education on the part of each individual. Compare the natural uneducated "tastes" of a child with those of an adult. The child will in all cases select the most sapid substances, and eschew the insipid ones as a rule, much to the horror of its parents. Moreover, if a child be allowed to exercise its own uninfluenced choice, it will almost invariably be in the direction of a substance having an acid reaction. It is the parents who, by constant training and insisting upon other articles of food being eaten, eventually produce in the child a tolerance of insipid substances.* This is done in all good faith by the parents for the child's health; it is prevented from eating substances which they know would in themselves produce gastralgia and dyspepsia. But it is entirely a gratuitous assumption that a like result would be produced in a healthy child by the consumption of such articles in amount proportionate to its age.

The digestive secretions of the parent have become vitiated and less active through long years of depression or maltreatment. Those of a normal child are in a natural condition, and adapted to deal with food in a natural form. I have frequently noted that very young children will deal with acid substances like fruit and salad quite successfully, yet a proportionate amount consumed by adults causes considerable discomfort.

It is within the knowledge of all, too, what enormous proportions of comparatively sour fruit may be consumed by the schoolboy with impunity.

If the salivary glands are in a state of proper development

* The following sentence in a child's story-book unconsciously reflects a real physiological principle: "It was not so much the cake, as the cherries in the cake, that Norah liked." 
and excitability, all the ordinary natural acids should be rapidly nentralized -if not before being swallowed, at least almost immediately afterwards, by the increased after-flow of a highly alkaline saliva. Not only is the "education" of the reflex salivary mechanism neglected by excluding them, but salivary secretion is actually depressed by the very much increased consumption of such articles as tea and meat. There is no doubt that children at the present time consume enormously more of these salivary depressants than they did a hundred and fifty years ago, when teeth were comparatively healthy.

From a dental point of view alone, neither tea nor meat could, it need hardly be said, exert any harmful action by themselves; but it is when they are taken in conjunction with soft, adhesive, insipid carbohydrates, as they usually are, that the harm results: even the feeble natural protection evoked by the latter is still further decreased.

Compare the dietetic conditions in England, say, one hundred or two hundred years ago, when caries was quite a spasmodic disease, with those obtaining to-day. Then there were no elaborate roller mills for the ultra-refinement of flour: bread was very largely "home-made" from flour ground between stone rollers. Then there was no wholesale importation of meat from abroad, there were no enormous biscuit and chocolate factories pouring out hundreds of tons of tooth-destroying material every year; tea and sugar were both articles of luxury and very dear.

The practically universal drink at all meals was wine, French and Spanish being drunk by the upper classes, and English wine-i.e., cider-being the drink of the middle and lower classes; and these, of course, were active salivary stimulants. Pepys wrote in I660: "I did send for a cup of tee (a China drink), of which I had never drank before." England now receives from India and Ceylon alone 300,000,000 
pounds of tea per annum, and the consumption per head is between 5 and 6 pounds per annum.

The manufacture and consumption of confectionery has developed enormously of recent years, and in this class of comestible easily fermentable sugars are largely used, not the least important of which is honey. The production of honey for this purpose is conservatively estimated at 300,000 tons per annum. Again, if eaten alone honey would not be anything like so harmful, as it is when incorporated with finely pulverized and cooked starches.

The total consumption of sugar per head for the United Kingdom is estimated at $77 \cdot 83$ pounds per annum, and, seeing that nearly the whole of this is probably uncombined with an acid, it all has a potential for initiating caries.

The method of making bread nowadays by chemical aeration conduces also to caries. In the old-fashioned method yeast more or less pure was used in the "leavening " process, and also a not inconsiderable amount of salt (as is the custom in Scotland at the present time), the result being that the bread had a distinctive flavour of salt and a just appreciable acidity due to the presence of acetic and lactic acids. Now, however, since "aeration" is produced by the action of acids on carbonate of soda, and since excess of the latter is always added to prevent sour taste, the resulting reaction is always alkaline, and therefore the bread is a salivary depressant.

In order to have some definite facts as to the average food of a modern civilized community, I collected the detailed dietaries of I,500 public-school children on one day. The age of the children varied from ten to fourteen years. The district was one in which there is practically no poverty, in which, too, the living is known to be good amongst all classes, yet it is one, also, in which caries is known to be present in over go per cent. of the children. 
The following tables give in concise form some of the results of the inquiry; they are self-explanatory:

DIETARIES OF FIFTEEN HUNDRED SCHOOL-CHILDREN.

Breakfast.

Solids.

Article of Diet.

Bread and butter . .

Porridge .. ..

Eggs

Toast

Bacon (or meat) .

Jam

Cake or biscuit

Fruit

Potatoes

Pudding

Tea .

Milk

Cocoa

Water

Coffee

Lemonade .

Neat

STER.

Pudding or pie

Solids.

Vegetables .

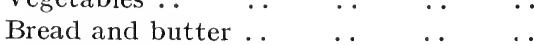

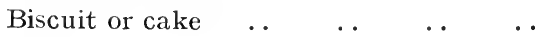

Percentage of Meals in which it formed a Part.

$64 \cdot 4$ $53 \cdot 8$ $47 \cdot 8$ $37 \cdot 8$ $33 \cdot 4$ I0.6 $5 \cdot 0$ $\mathrm{I} \cdot 6$ $\mathrm{I} \cdot \mathrm{O}$ 0.5

Fluids. Jam

Eggs

Fruit

$\begin{array}{lllr}\ldots & \ldots & \ldots & 75 \cdot 0 \\ \ldots & \ldots & \ldots & 6 \mathrm{I} \cdot 6 \\ \ldots & \ldots & \ldots & 55.6 \\ \ldots & \ldots & \ldots & 40 \cdot \mathrm{I} \\ \ldots & \ldots & \ldots & \mathrm{I} 9 \cdot 6 \\ \ldots & \ldots & \ldots & \mathrm{I} 0 \cdot 0 \\ \ldots & \ldots & \ldots & 4.8 \\ \ldots & \ldots & \ldots & 4.2\end{array}$

Tea..

Fluids.

Vater

Milk

Soup

Lemonade .

Cocoa

Coffee

\begin{tabular}{|c|c|c|c|}
\hline$\ldots$ & . & $\ldots$ & $31 \cdot 6$ \\
\hline . & $\ldots$ & $\ldots$ & 28.6 \\
\hline . & $\ldots$ & $\ldots$ & $14 \cdot 6$ \\
\hline$\ldots$ & $\ldots$ & $\ldots$ & $7 \cdot 6$ \\
\hline$\ldots$ & $\ldots$ & $\ldots$ & $7 \cdot 6$ \\
\hline . & . & $\cdots$ & $5 \cdot 6$ \\
\hline . & $\ldots$ & $\ldots$ & 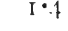 \\
\hline
\end{tabular}


TEA.

Solids.

Article of Diet.

Percentage of Meals in which it formed

Scones, cakes, or biscuits a Part.

Bread and butter. $76 \cdot 8$

Meat

$68 \cdot 3$

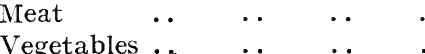

$49 \cdot 2$

Pudding or pie .

$34 \cdot 2$

Jam

$26 \cdot 0$

Eggs

$19 \cdot 7$

Fruit

Salad

Toast

Cheese

Fluids.

$\begin{array}{llllllr}\text { Tea .. } & \ldots & \ldots & \ldots & \ldots & \ldots & 65 \cdot 8 \\ \text { Milk. } & \ldots & \ldots & \ldots & \ldots & \ldots & \text { I I } \cdot \text { I } \\ \text { Water } & \ldots & \ldots & \ldots & \ldots & \ldots & 9 \cdot \mathrm{I} \\ \text { Cocoa } & \ldots & \ldots & \ldots & \ldots & \ldots & 6 \cdot 5 \\ \text { Soup } & \ldots & \ldots & \ldots & \ldots & \ldots & 3 \cdot 0 \\ \text { Lemonade } & . & \ldots & \ldots & \ldots & \ldots & \mathrm{I} \cdot 5 \\ \text { Coffee } & \ldots & \ldots & \ldots & \ldots & \ldots & \mathrm{I} \cdot 3\end{array}$

SUPPER.

Solids.

Bread and butter ..

$\begin{array}{lllr}\ldots & \ldots & \ldots & \text { I } 5 \cdot 9 \\ \ldots & \ldots & \ldots & \text { I } 4.8 \\ \ldots & \ldots & \ldots & \text { I } 3 \cdot 0 \\ \ldots & \ldots & \ldots & 10 \cdot 3 \\ \ldots & \ldots & \ldots & 3 \cdot 4 \\ \ldots & \ldots & \ldots & 1 \cdot 2 \\ \ldots & \ldots & \ldots & \mathrm{I} \cdot 2 \\ \ldots & \ldots & \ldots & 0.7 \\ \ldots & \ldots & \ldots & 0.6 \\ \ldots & \ldots & \ldots & 0 \cdot 0\end{array}$

Cake

Biscuit

Chocolate ..

Meat

Fish and potatoes.

Salad

Porridge

Fruit

Fluids.

Milk. .

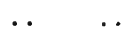

I $2 \cdot 6$

Water

Tea ..

Coffee

I $2 \cdot 4$

Lemonade 
In cases where no supper was taken, the last thing eaten at tea-time is recorded in the following table:

\begin{tabular}{|c|c|c|c|c|c|c|}
\hline & $\ldots$ & $\ldots$ & . & \multicolumn{2}{|c|}{ I 6.80 per cer } \\
\hline & & . & . & .. & $13 \cdot 30$ & ,, \\
\hline \multicolumn{2}{|c|}{$\begin{array}{l}\text { Bread and butter } \\
\text { Pudding } \quad .\end{array}$} & . & . & $\ldots$ & $5 \cdot 40$ & , , \\
\hline \multicolumn{2}{|c|}{ Bread and jam } & . & $\cdots$ & . & $4 \cdot 60$ & ", \\
\hline \multirow{2}{*}{\multicolumn{2}{|c|}{$\begin{array}{l}\text { Biscuits } \quad . \\
\text { Meat and potatoes }\end{array}$}} & . & . & . & $2 \cdot 20$ & ," \\
\hline & nd potatoes & . & . & $\ldots$ & $I \cdot 20$ & ," \\
\hline \multicolumn{2}{|c|}{ Fruit $\ldots \quad \ldots$} & . & . & .. & $0 \cdot 80$ & , \\
\hline \multirow{2}{*}{$\begin{array}{l}\text { Tea } \\
\text { Soup }\end{array}$} & . & $\ldots$ & $\ldots$ & $\ldots$ & $0 \cdot 30$ & ," \\
\hline & . & . & . & . & $0 \cdot 06$ & ," \\
\hline
\end{tabular}

It cannot be suggested that these tables do not show an extremely liberal dietary; it is possible that in some cases a little more than the truth was told; but for the vast majority of the cases I am satisfied that these tables represent faithfully the dietary of the district. Such diets might be criticized from other points of view than the solely dental, especially bearing in mind that it is the food of children which is being considered.

Note, for instance, the enormous proportions of meat and tea which are consumed, loading up the systems of the children with alkaloid and extractive stimulants.

From a dental point of view, the diet may be very briefly summed up as being salivary depressant and of high acid potential.

A most remarkable similarity will be noted between the order in which the articles of diet are arranged in these tables and the order previously noted of their capability of acid-production. They are also practically in inverse order to their power as salivary stimulants.

If nothing were known about the teeth of these children, it could be said with absolute certainty from these data that they would be found to be in an extremely bad condition. This, as has already been indicated, is so; moreover, and I speak from a considerable experience in examining the teeth of the children of this district-the incidence of caries in each 
mouth is appallingly high, so much so as to constitute a disgrace to any well-to-do civilized community.

One of the points of most importance is the food last eaten during the day, and therefore, in the majority of cases, débris of which would remain to be fermented during the night.

It is seen that bread and butter, cake, biscuit, and chocolate, are the articles which are last consumed in the great majority of cases-articles which adhere strongly to the teeth, form the greatest amount of acid, and produce the least flow of saliva.

It is, again, one of the most significant facts brought out by this inquiry, that not one child in fifteen kundred had fruit of any kind last thing at night, and that only 0.8 per cent. had fruit as the last thing at tea-time.

In view of the fact that fruit has been shown to be one of the best detergents and excitants of the solvent and neutralizing powers of saliva, its practically complete absence here cannot be regarded as other than one of the greatest causes of the prevalence of caries.

Although, naturally, diets vary in different localities and countries, and vary also amongst different classes in the same districts, yet it probably would not be wrong to take the foregoing tables as representing an average dietary of modern civilization, erring, probably, on the side of liberality; but, still, the proportions of substances to each other for various meals (excepting, perhaps, meat and tea) would, I think, practically hold good. We may therefore conclude that the dietary of civilized communitics at the present time errs seriously in the direction of being too soft, of having too high an acid potential, of being not sufficiently stimulating to the salivary glands and not sufficiently detergent.

In considering how such a dietary may be corrected, social and economic conditions have to be borne in mind; it is neither possible nor desirable that any sudden or drastic 
change should be made. Conditions must be accepted as they are found, and an attempt made to improve them.

It is impossible, for instance, under modern conditions to ban absolutely all soft and fermentable carbohydrates. Improvement in this direction may come in time, but it will be essentially a slow and gradual process, depending upon the rapidity with which people are educated to demand such an alteration.

Sufficient has, however, been said to show how the majority of food substances of high acid potential may be rendered practically innocuous.

It has been shown that by the combination of strong salivary stimulants with such substances, either in a mixture or, much better, in sequence, the acid-production may be reduced to a minimum or entirely negatived.

What is therefore to be advocated is that all meals should contain a fair proportion of salivary excitants, and, more important still, should both commence and end reith some article of diet having an acid reaction.

This does not of necessity imply a "vegetarian " diet; in fact, many "vegetarian" meals are distinctly conducive to caries on account of their soft, pappy, and adhesive nature.

The only meal in which the principle of salivary stimulation is usually correctly observed at the present time is a dinner commencing with an hors-d'xuvre and terminating with fruit as dessert.

A similar arrangement, however, can be quite easily managed for all other meals. When garden fruit and vegetables, particularly the "salad" variety, are in season, this should present no difficulty whatever, and the length of "season" is constantly being increased by the importation of fruit and vegetables from warmer climates; in fact, at all seasons of the year now, and practically everywhere, some fresh fruit at least is available. 
For breakfast, porridge with salt may form the initial stimulant. This may be followed by anything else customary to the individual, such as fish, bacon, bread and butter, and marmalade or jam; the latter should have a distinctly acid flavour, and should not be merely syrup flavoured with fruit-juice, as too many of the factory-made articles are. The meal should be concluded with some form of fresh fruit-orange, apple, banana, pineapple, pears, plums, etc.-preferably raw, but they may be "stewed."

For luncheon or the midday meal, the initial stimulus may be given by some modified form of hors-d'xuvre, such as a radish or a small portion of some acid fruit.

With meat some form of sauce may be taken; such things, being nearly all acid, serve as excellent salivary stimulants. (There is no objection to children taking small quantities of these sauces. I have never seen any ill results follow.). The meal may be concluded with stewed fruit, fruit pudding or pie, provided that in the latter the fruit predominates, and not the starchy element, and that it is not made too sweet. "Salad" should, of course, be taken wherever possible, but its good effect may be entirely neutralized by its being followed, as it too often is, by a biscuit and coffee or some form of confectionery and tea.

Tea, especially when it is the last meal of the day, is the one most responsible for the production of caries. It almost invariably ends with cake and a second or third cup of tea, containing probably, in the vast majority of cases, a high percentage of tannin. Thus absolutely ideal conditions are brought about for acid fermentation to take place in the mouth. There are a number of salivary stimulants which may be included in this meal, such as tomato, cucumber, cress, sardines, shrimps, or fruit sandwiches. Fruit salad or any fruit in season should terminate the meal.

Last thing at night, children (and adults too) should always 
eat a small portion of some detergent and acid fruit, such as orange, apple, pear, or pineapple.

Taken in such a manner, the total amount of fruit and vegetable need not be large, and in no case should such a dietary be commenced suddenly. It should, especially in those who have been unaccustomed to the inclusion of fruit and salad in their dietary, be worked up to gradually, by commencing with one meal a day; then, after a week or so, two meals may be terminated with "acids," and finally the principle may be extended to all meals.

Should any intestinal irritation be set up, the inclusion of boiled milk in the dietary will correct it; this I have found to be but very occasionally necessary, and only in the initial stages.

The much-dreaded bugbear "diarrhœea" is much more likely to be caused by eating fruit in an unclean condition than to be due to the mechanical or chemical action of the fruit on the bowel. All fruit, therefore, especially that of unknown source, should be well washed or have the skin removed before being eaten. The only effect of fruit taken regularly and in such moderate quantities is to insure a full and regular action of the bowels.

In special cases the reaction and amount of saliva should be estimated during the change, to see that the desired result is being produced; and when the maximum amount and alkalinity per minute have been reached, any increase in the acidity of the dietary should be stopped, and it may even be decreased a little.

The difficulty, in many cases, of adopting such a dietary may be on account of expense and inaccessibility of fresh fruit and vegetables. Tinned fruits are, however, now everywhere available at moderate cost; and these, especially pears, pineapple, and apricots, may be substituted, not with advantage, but as being better than nothing. 
In respect to fluids, children particularly should never drink tea, and the less it is consumed by adults the better, even from a dental point of view alone.

Milk, water, home-made lemonade, and weak lime-juice, are the beverages suited for children. Lemonade should not be too strong, and should contain a sufficiency, but not an excess, of sugar; that is to say, the acid should be quite appreciable to the child, and should be sufficient to precipitate the mucin and prevent the sugar from being fixed to the teeth.

If similar beverages were more consumed by adults, it would, I think, largely lessen the craving for tea and sweetmeats so prevalent at the present time.

As regards sweets, all are good in that they are salivary stimulants, and all are bad in that they are readily fermentable; and as a general rule it may be said that the ill effects quite outweigh the good effect. They all (practically) leave sugar behind in the mouth to be transformed into lactic acid - chocolates and caramels most, and hard-boiled sweets like toffee least. The old-fashioned "acid drops" are possibly the least harmful. Children should, however, be encouraged to spend their pocket-money on fruit rather than on sweets, and on biscuits least of all. Almost all children prefer fruit to sweets if they are allowed to exercise their choice. Of the I,500 children whose dietaries I examined, 90.35 per cent. said they preferred fruit to sweets, and only 9.65 per cent. preferred sweets; yet the amount of sweets consumed by these children was enormous, and the amount of fruit a minimum. I think there is no doubt that a growing child can obtain all the sugar it needs for its muscles, fat, and heat development, from consumption of it in a natural form-i.e., as found in fruit and some vegetables.

Bunge* has called attention to the danger of the great increase in the consumption of pure sugar in recent times,

* Quoted by R. Hutchison, "Food and Dietetics," p. 280. 
pointing out that it must diminish the amount of vegetable foods in the diet, thus leading to a loss in the supply of calcium, iron, fluorine, and other mineral ingredients to the blood. Hutchison is also of the opinion that it may be connected with the increased commonness of diabetes.

It is commonly affirmed in textbooks that one of the articles which have been quoted as salivary stimulants-i.e., cider-destroys the teeth. With this the author cannot agree, having had considerable experience of the teeth of the inhabitants of one of our cider counties, and also of those of other counties and countries. The difference between dentures from this county, and those from an adjoining county where mining was carried on, and fruit and cider were not available, was always most marked and obvious; and whenever the mining community makes an excursion into the cider country, their avidity for fruit is, to say the least, very pronounced.

It has been stated that the consumption of large quantities of grapes in those patients undergoing a "grape cure" has bad effects upon the teeth. I have no knowledge of this; but if so, it is probably attributable either to the grapes being too ripe and hence acting, as has been shown, as very feeble salivary stimulants, or, if not too ripe, to the constant presence of too much acid in the mouth proving more than the saliva can neutralize; possibly the glands get overworked, and do not have time to recuperate.

Black* some years ago came to the conclusion, after " a hasty examination" of dried skulls, that there was a connection between immunity to caries and sour-fruit eating, but apparently abandoned or did not follow up his conclusion; and Leon Williams $\uparrow$ states that the Sicilians, who are large consumers of lemons, are particularly free from caries.

* “American System of Dentistry," p. 730.

† Quoted by Goadby, "Mycology of the Mouth," p. I 39. 


\section{Note on the Soured-Milk Treatment.}

However beneficial this treatment may be for other portions of the alimentary tract, there can be no doubt that as a general rule it must be harmful to important organs of the first part of the tract-i.e., the teeth. Not because the milk is sour-in that respect it can be only beneficial-but because a large number of lactic acid organisms are introduced into the mouth, and these of a variety specially selected on account of their ability to form a maximum amount of acid. The following tests with soured milk give examples of what may happen:

Two persons, A and B, drank ordinary milk, and then each insalivated 2 grammes of bread; this was incubated for fortyeight hours, and the acid-formation estimated-

$$
\begin{aligned}
& \mathrm{A}=2 \cdot 83 \text { units }\left(\frac{\mathrm{N}}{5} \mathrm{NaOH}\right) . \\
& \mathrm{B}=2 \cdot 8 \text { ", }
\end{aligned}
$$

Milk which had been infected with lactic-acid bacilli (fermenlactyl) for eighteen hours at body temperature was then drunk, and 2 grammes of bread again insalivated by each. In forty-eight hours the acid-production was-

$$
\begin{aligned}
& \mathrm{A}=4.6 \text { units. } \\
& \mathrm{B}=4.7 \quad,
\end{aligned}
$$

This considerable increase in both cases could only have been due to a considerable increase in the initial number of organisms present in the mixture, and there is no reason to doubt that the figures represent the proportionate tendency to tooth destruction in both cases.

Both of the above individuals were susceptible to caries; a similar test was made with an immune person:

After ordinary milk, the bread yielded in forty-eight hours I.4 acid units.

After soured milk the bread yielded in forty-eight hours I. 2 acid units. 
The difference between this and the two former tests is very marked; not only is the acid-protection from the control only one-half of what it was from the susceptibles, but also the amount of acid produced from the bread masticated after soured milk was less than the control. The results are to be explained entirely, I think, by the increased neutralization by the saliva of the immune. It therefore seems clear that in the average individual-i.e., one susceptible to cariessoured milk may prove to be very harmful to the teeth. In order to combat this the teeth should be cleaned, and a salivary stimulant used immediately after taking the milk (or powder). To expect to remove the introduced organisms by rinsing the mouth out with water for half a minute or so would be futile. I would suggest the eating of a piece of orange as one of the simplest and best prophylactic measures which could possibly be adopted. 


\section{CHAPTER XX}

\section{EDUCATIONAL MEASURES}

THERE can be no doubt that ignorance is largely responsible for the present widespread prevalence of dental cariesignorance of the cause, ignorance of a remedy, and ignorance of the gravity of the condition and the secondary consequences. Ignorance chiefly on the part of the general public, but not entirely; alas! also, to a greater or less extent, on the part of physicians, surgeons-and dental surgeons, too.

It is clear that, in order to obtain any appreciable diminution of the incidence of a disease which is afflicting over 90 per cent. of the population, knowledge of the cause and seriousness of the conditions cannot be too widely disseminated, nor the general adoption of the utmost prophylactic measures available be too insistently urged.

There are three classes who have the power of propagating this knowledge in especial degree. They are the teachers in primary schools, medical men, and dentists.

\section{The Teachers in Primary Schools.}

Educationists are coming more and more to recognize the truth of the well-worn adage, Mens sana in corpore sano, and are devoting more time and attention to the attainment of that end. Hygiene and physical drill are taught, cooking is taught, and free meals are provided. But these will benefit nothing if the child is suffering from septic absorption arising from diseased teeth. Wallis* has found that the children with the most marked oral sepsis were always at the bottom

* Wallis: "The Necessity of Dental Clinics in Connection with Elementary Schools," British Dental Journal, i908, p. 861. 
of their class. It is therefore to the interest of education, and of those teachers who desire to see the mental capacity of their pupils developed to the greatest extent-under the best conditions for their profiting thereby-that considerable attention should be paid to the prevention of dental caries.

The mere examination of the children occasionally by a medical man or a dentist, and the being advised that their teeth require treating, is utterly insufficient; an occasional explanation, perhaps by the school medical officer or school dentist, of the means and necessity of prophylaxis, is likewise absolutely inadequate. What is necessary is persistent, regular, oftrepeated, definite lessons in oral hygiene, and the only persons in a position to carry out such work are the teachers themselves. Such definite and regular lessons should be given to the children in each standard separately. The lessons should be graded according to the standard and understanding of the pupils.

In order to stimulate the interest and make it a real lesson to the higher standards, it will be necessary to go into much more detail than would be possible or desirable for children in the lowest standards; but no child should be too young to receive some definite instruction.

Children should be made to realize the value, physiological and financial, of sound teeth. Reduced to its most sordid aspect, the loss of all tooth substance means the expenditure sooner or later of money, or its loss through incapacity. By ascertaining the average weight of teeth, it is estimated that each tooth is worth to its possessor the monetary value of an equal weight of gold at least; that each part of a tooth dissolved by lactic acid is equal to so much gold taken out of the individual's pocket-not voluntarily, but with absolute certainty, and involuntarily.

Occasional lessons to a whole school on such a subject would not excite much attention on the part of the pupils, and in a 
few weeks at most all the details of the teaching would be quite forgotten. There should be at least one or two lessons given in each term in each standard; and then by the time a child has passed through all the standards it may be hoped that some of the main principles of oral prophylaxis will have been really learnt, will be put into practice, and the effect last until such time as the child becomes in turn a parent; for, after all, it is the parents who have the power and the will to largely reduce the prevalence of caries. But, of course, parents qua parents are difficult to reach by educational means; it is therefore from the proper instruction of the potential parent that we have most to expect.

In order that such work may be carried out, and in view of its national importance, the teachers must themselves first receive instruction. There should be appointed to all training colleges a dental surgeon, who should deliver a short course of six lectures annually to the students there being trained for the teaching profession, and a certificate of having attended such lectures to the satisfaction of the lecturer should be absolutely obligatory. Further, all dental associations should arrange for lectures to be delivered locally to those teachers who have previously received no such instruction. (Such lectures should not be purely honorary, but should be sufficiently well paid to insure their being well prepared and well presented.)

For the purpose of instructing both pupils and teachers, diagrams-as many as possible-are a sine qua non. The visual centres of the average modern brain are far more retentive than the auditory, and every advantage should be taken of the fact. The diagrams should be simple and bold-should be diagrams, and not pictures, far less actual photomicrographs. It should be remembered that we are dealing with people untrained in differentiating minute histological details, and what is required is that an impression 
should be created, and a lasting impression-such that a mental picture of any important diagram could be visualized at any time. Stress is laid upon this point, because many of the diagrams intended for school use at the present time are far too complicated and technical for a lay mind, and for a child's grasp in particular.

The teachers, also, of the cooking classes held for the senior girls in many schools should recognize that by their teaching they can accomplish much for the good or ill of the teeth of the people. There should be no difficulty at all in treating even simple and inexpensive dishes so that they may contain salivary stimulants, nor in arranging them in proper order, that the most fibrous and more acid constituents of a meal may come last. Much may here be done to educate the gustatory nerves, to cultivate in the children a taste for tasteful things (other than confectionery); to stimulate their interest in, and to point out the necessity of, as large a variety of fresh vegetables, salads, and fruits, as possible; and, on the other hand, to teach them to avoid tasteless, soft, sticky, and simply sweet articles of diet, and so on.

But here again, in order that the teacher might work rationally and enthusiastically, it would be necessary that she should first of all understand thoroughly the physiological principles underlying such measures, and should appreciate the urgency of their adoption. She should appreciate, too, that by stimulating the glosso-pharyngeal and lingual nerves, not only will the teeth be protected, but the gastric juice be psychically stimulated, and the meal therefore better digested.

The students, therefore, in schools of cookery should receive instructions also in the cause and prevention of dental caries; and the Board of Education authorities should recognize the importance of such knowledge, and the serious responsibility of teachers of cooking in disseminating knowledge which may be for good or for ill in the health of the nation. 


\section{Medical Practitioners.}

Medicine and dentistry have been most closely associated since the very earliest times; in fact, down to the seventeenth century dentistry was practised almost exclusively by physicians. Dentistry then began to be practised by men who had not been trained as physicians or surgeons, and their numbers have gradually increased from then until now. This is in itself a matter for regret, but what certainly is a matter for much more regret is that, correspondingly, interest in the teeth on the part of physicians and surgeons has gradually diminished, until it has fallen almost to zero. There is too great a tendency to regard the teeth as isolated parts of an organism, as organs which exist of themselves and for themselves, and are not intimately linked up in function with the rest of the body. It is not sufficiently recognized that the teeth are an integral and inseparable and most important part of the alimentary system, both from a physiological and pathological point of view. In consequence of this the amount of training and instruction a medical student obtains at the present time in the diseases of the teeth is almost negligible. The result is that a medical practitioner is, by overlooking the teeth, liable to overlook the origin of the disease he may be treating; but this, serious as it may be, is not the most serious thing that results. A medical man has almost unrivalled opportunities for impressing upon parents the necessity for preservation of the teeth by dietetic and prophylactic means; but if as a student he learnt nothing of dental physiology and pathology, nor of the treatment of diseases of the teeth, he is not in a position either to seize the opportunity or to act upon it. The advice of a medical man on the condition of a child's health is usually sought many years before the child is taken to a dentist, yet not perhaps before signs of dental caries have begun to manifest themselves; and it is 
at this period that sound advice is so valuable, and its omission so far-reaching in its effects. There can be no doubt that the constant and systematic omission of reference to the teeth by general practitioners has had a great deal to do with the apathy with which the majority of the laity regard dental disease. The ordinary individual argues that if the condition of the teeth were important, or had any relationship to other diseases of the body, the doctor would have been sure to mention it. If on the other hand the doctor in examining his patient never omitted the teeth, and always upon finding signs of disease of those organs gave advice as to prophylactic treatment, and insisted as far as possible on this being carried out, how different in a very short time would be the opinion of the public as regards the importance of the preservation of healthy teeth !

"In those cases in which some advice is given, it is usually " to clean the teeth" or "to visit the dentist"-quite sound in itself, but which, by falling far short of what is necessary, conveys an impression of unimportance. Dental caries is, as we have seen, in a large measure due to errors of diet extending over the first twelve years of a child's life, and it is just during that period that the general practitioner's advice is frequently requested as to the suitability or otherwise of articles of diet; or, at least, he has opportunities of pointing out authoritatively the deleterious effect of the common dietetic errors from a dental point of view. We cannot, therefore, avoid the inference that, if such had been the universal practice, the incidence of caries would not now be anything like so high. In order to avoid such a stigma in future, medical students must be instructed in the care of the teeth. A medical student, before he qualifies, should have some knowledge of what diet is beneficial and what deleterious to the teeth, and he should be taught to put this into practice. In doing so he will only be fulfilling the 
general principles of correct dietetics, for it might be stated in general terms that "what is best for the teeth is best for the rest of the alimentary system." He should also know something of how to aid natural resistance to caries by artificial prophylaxis; he should be able to write suitable prescriptions for various mouth-washes, dentifrices, etc., for varying conditions of the mouth; he should be able to instruct parents in the care of their children's teeth. All this and more he should know.

He is taught along similar lines with regard to the eye, the ear, and throat; why, then, should the teeth and mouth be omitted ? He should, in fact, attend a short course (theoretical and practical) on stomatology, or diseases of the teeth and mouth, and having some precise knowledge of such matters he will no longer think them unimportant.* He will then be in a position to render valuable service to his patient; to himself, in the saving of his time in the diagnosis and treatment of many cases; and moreover, and above all, to the community as a whole, by directing attention to the necessity of adopting stringent but rational methods for the prevention of dental caries.

\section{Dentists.}

If, during the past one hundred years, half as much time, money, and brain power, had been spent in evolving means for the prevention of dental caries, as has been spent in the perfecting of ways and means for replacing artificially tissue lost by disease, there can be no doubt that the present condition of affairs would not have come about. In fact, it is probably not too much to say that the majority of the members of the dental profession have been content, not to treat

* Such a course need not add any time to the curriculum. One hour a week during the final summer session can be found without difficulty, and, from personal experience, I find students are only too willing to attend such a class. 
the disease at all from a seriously preventive point of view, but merely to make good, as far as possible, the destruction produced during the progress of the disease.

Unfortunately, the training at most of our schools and hospitals must be blamed for this. If one may judge from personal experience, and from the amount of space devoted to prophylactic treatment in textbooks, the extent of a student's teaching in this subject is somewhat limited. An examination of the six best-known textbooks on dentistrythree English, two American, and one German-shows that on an average $0 \cdot 16$ per cent. of a book is devoted to the preventive treatment of dental caries; yet no one would suggest that this proportion in the least degree represents the importance of the subject.

The dental student, whilst at hospital, should have full facilities and time for learning all that can be taught him in this branch of his work; it should not be looked upon as a subject which can be " picked up " anyhow, or as one which it is not necessary to know much about before qualification, and which may then perhaps be relegated to the attention of a few "faddists." There should be special lectures by a suitable lecturer on preventive dentistry; this should be supplemented by a practical laboratory class where experiments and observations bearing upon the prevention of caries could be made by students. The student would thus come to know the facts of the subject, instead of having a more or less empty belief in them only. Students should also attend a special clinic for the treatment of cases by prophylactic means. They should be taught to go into each individual case very thoroughly, to ascertain wherein lies the cause of caries in any particular case; to find out which part of the line of defence has broken down or is too weak, or, on the other hand, what factors in the attacking forces are increased in amount or virulence. When this has been ascertained 
by means of accurate, visual, chemical, and bacteriological observations, the student should be taught to outline a course of treatment for the patient-to draw up a dietary, and write prescriptions, and give instructions for artificial prophylaxis, suitable to the case.

There is one other matter in which the dental profession and the teaching of dentistry in Great Britain lag behind. No provision is made in any of our hospitals, schools, or Universities, for the proper remuneration of teachers of dentistry or dental subjects; consequently, except in a few cases where special grants are made, no teacher of dentistry can possibly spend a great deal of his time in research work. In medicine full facilities are offered for research and progress in the treatment of a number of diseases, at Universities and hospitals-in dentistry practically none. There can be no doubt that until Universities and governing bodies recognize the importance of the subject, and create " chairs" whereby the holders may be justified in devoting the whole of their time, energy, and enthusiasm, to teaching and research work, the teaching will continue to be of a spasmodic and disconnected character. 


\section{THE NEED FOR SPECIAL LEGISI.ATION}

THERE can be no doubt that the gravity of the disease with which we are dealing is not recognized by the laity, and therefore not by Governments. The extent of the disease is beginning to impress itself upon authorities now that the examination and possible treatment of school-children is being undertaken at the public expense. So far, however, the cost involved in the treatment of dental caries by " fillings " on a universal scale has appeared to be so great as to hinder any other than isolated efforts in this direction. But even now that it is beginning to dawn on authorities and public bodies that 90 per cent. of the children have carious teeth, the seriousness of what this means, the gravity of the ills to which the condition is precedent, is not in the slightest degree appreciated.

It is perhaps agreed that "bad teeth cause indigestion," and that is as far as the sequence of events is followed. This is bad enough in itself, for how can it be hoped to rear a nation of mentally and physically strong individuals if, during the periods of growth and development, there is indigestion, and therefore non-assimilation of food? Added to this is the slow, insidious, but nevertheless sure, chronic septicæmia, brought about by the constant ingestion of toxins, pus, and bacteria, from a mouth containing numerous suppurating foci. It must be recognized, too, by the public that anæmias of severe type are frequently induced in the same manner; that tubercular glands of the neck, serious 
forms of Ludvig's angina, frequently fatal, are also caused by diseased teeth; that malignant disease (cancer) of the jaws, tongue, and lips, often originates solely from diseased teeth, and from this cause alone a large number of lives is lost annually; that a large number of disorders of the adjacent parts-i.e., muscles, bones, nose, eyes, and ears-are intimately associated with diseased teeth, either reflexly through the nerves or by direct extension of the morbid process; that in conditions of unstable cerebral equilibrium the presence of diseased teeth is frequently sufficient to excite an attack of nervous disease, such as epilepsy, tic (spasms), hysteria, and some forms of insanity; that in addition to all these ills, of which large numbers of cases have been, and are constantly being, recorded, there is a chronic lassitude, lack of appetite, mild headache, constipation, and an inability to perform a normal amount of work, almost invariably associated with extensive dental disease. It is this relationship between cause and effect which requires to be driven home in the public mind. The disease itself is not always obvious, and the channels by which its effects spread are not recognized.

If the disease were more obvious, there is no doubt that long ago the public mind would have been stirred, and legislation would have been passed to facilitate the checking of such universal suffering. Suppose, for instance, every alveolar abscess pointed on the face instead of in the mouth, the hideous disfigurement resulting therefrom would long ago have arrested attention; yet probably in this case the harm to health would be infinitely less, because the products of disease would be to a certain extent outside the body, and not, therefore, absorbed into the system.

Suppose a similar condition affected the finger-nails-suppose that $9^{\circ}$ per cent. of the community went about with decayed or suppurating finger-nails-the idea would long ago have been so revolting that extensive measures would have 
been adopted for the suppression of such a disease; yet the total systemic disorder and the annual loss of life would have been far less than it is from dental disease.

The report of the Royal Commission on Physical Deterioration found that dental caries was a potent cause of physical deterioration, and Osler is of the opinion that in this respect the effect of dental disease is greater than that of alcohol. On humanitarian grounds alone, then, action is urgently required to suppress the advance of this most prevalent of all diseases. The principle has been recognized in nearly all communities now, that the Government is responsible in the main for the health of the nation; and in cases of serious epidemics, of widespread toxic effects, of disease affecting large proportions of the people, legislation is provided enabling authorities to cope with the evil. Surely a disease affecting 90 per cent. of the population, and as far-reaching in its effect as this has been shown to be, is a national ill, and demands legislative measures. Legislation has been enacted for diseases which affect a far less percentage of the population, and from which the loss in national health and wealth is far less. Take, for instance, the laws controlling and limiting such diseases as phosphorus-poisoning, leadpoisoning, prohibitions on the sale of alcohol and opium. But the effects of these are obvious, and therefore have secured attention and a large measure of suppression.

Legislation is demanded upon economic grounds. It must be perfectly obvious that any disease or factor which deleteriously affects the majority of the workers and wealthproducers of a nation is one which demands the serious consideration of legislators.

At the very lowest estimate, the annual economic loss in Great Britain alone from this cause cannot be less than a million and $a$ half sterling. This is based upon the assumption that the average extent of caries is three teeth per individual, 
and that each of these teeth causes one hour's loss of time and work per annum, and that the average value of this time is as low as threepence per hour. This works out at a sum of $\ell_{\mathrm{I}}, 687,500$, the population being taken at 45,000,000. As a matter of fact, the real sum must be far in excess of this, if we take into consideration the prolonged economic loss incurred by the secondary occurrences of malignant disease, tubercular glands, pernicious anæmias, cerebral disorders, and the chronic debility mentioned above. The fact, also, that many of the people so afflicted become a positive charge on the community by occupying space, time, and attention in hospitals, workhouses, and asylums, tends further to increase the national loss.

There can be no doubt whatever that a very large proportion of the cases occupying the beds and attending the outpatient departments of our general hospitals are there solely because of primary dental disease, which has led to grave secondary disorders. The recovery, also, of many patients suffering from a number of diseases having other than a dental origin is seriously hindered and prolonged by the presence of a septic mouth. Especially in this respect are thousands of pounds wasted annually in the sanatorium treatment of phthisical patients, who either cannot masticate properly the food abundantly supplied, or in whose mouth it becomes incorporated with a quantity of septic and toxic material, the constant ingestion of which either prevents or considerably retards recovery.

\section{The Form of Legislation.}

It has been amply demonstrated that the prevalence of caries is due to the habitual consumption of "artificial" as opposed to " natural " articles of diet. Most of the forms in which starchy and sugary foods are taken at the present time can only be regarded as luxuries, and, moreover, as 
dangerous luxuries-at least as dangerous as alcohol, for instance; and, like alcohol, they should be taxed. The manufacture of sweetmeats, confectionery, and fine white flour, should be carried on only in bond; and all such articles should be subject to Excise, either when manufactured at home or imported from elsewhere. The amount of the taxation should be sufficient to appreciably diminish the demand for such commodities, and the moneys so collected should be applied to the cheapening of other equally nutritious and less harmful forms of food-not less harmful only, but actually preventive of dental disease. Particularly the culture, carriage, freight, and importation, of fruit and fresh vegetables (and of meat in some places) should be fostered and aided, so that such articles may come (especially fruit) to be regarded as common, ordinary, and necessary constituents of every meal, instead of being, as at present, regarded as unnecessary and too expensive for common and constant use. Such legislation might well accord with a "back to the land" or "closer settlement" policy, the desirability of which, on economic grounds, is so much advocated.

In addition, the preventive treatment of dental caries must be made one of the most important branches of the public health service. To initiate and organize a department to deal efficiently with this branch alone, would be a source of national econcmy.

The present system of inspection and advice is good and necessary, and is a step in the right direction; but by itself it is futile, and almost useless to stem the tide of this national disease. The proposed system of treatment by means of fillings and extractions on the most extensive and costly scale can hardly be much better; it is to be regarded as a policy of expediency rather than of principle. No universal method of treating disease can be recognized as being upon right lines; no enormous expenditure of public money for the treatment of disease can be justified unless that treat- 
ment strikes at the cause of the trouble, and gives some reasonable hope that the incidence of the disease will be materially lessened. If the simile may be pardoned, such schemes are as if an army of small boys were pelting a glass house with stones, and the owners, instead of attacking and dispersing the cause of the trouble, employed an equally large army of workmen to be constantly repairing the damage as it went on. There would be two inevitable results: the vigour and number of the attackers would increase daily, and the work of the workmen would deteriorate.

There is but one rational way of treating disease on a large scale-that is, by building up the resistance, active and passive, of the individual to the disease, and by removing any obvious source of disease. How this may be done in the case of dental caries we have already considered in detail, and it is along such lines that State intervention seems to be required. The national health demands legislation. The pain and suffering, the lowered mental and physical condition, of the little children cry aloud for help. The people, their parents, are for the most part blind and ignorant as to the cause and remedy. For the removal of this ignorance, for placing means for combating the disease within the reach of all, and for making disease-causing factors less accessible, the State must be responsible. 


\section{N D E X}

Abnormalities, iol

Abyssinian diet, 323

Acid, acetic, I9

aceto-acetic, $20 \mathrm{I}$

benzoic, 268

butyric, I 9

citric, 169

formic, I9

hydrochloric, I69

lactic, 19, 20, 22, 23

salicylic, 268

succinic, I9

sulphuric, I69

tannic, I68

tartaric, I69

Acid dietary, effect on saliva, I 70 recommended, 34I

drinks, 344

drops, 344

-forming bacteria, 20

potassium tartrate, and saliva, 167 as dentifrice, 267

sodium phosphate, I 27

Acids, effect on dialysis of saliva, 223

on enamel, 127

natural organic, 23 I

organic and inorganic, compared, I69

Adenoids, 49, 6o

Adsorption, I I 7

Africa, natural races of, caries, I2 diet, 322

Age incidence, caries, I 5

Albumin in saliva, I I I

Alcohol, 267

effect on taste, 177

Alimentary secretions, 295

vicious circle, 299

Alkalies, effect on dialysis of saliva, 223

Alkaline dentifrices, I 76 to be abandoned, 275
Alveolar abscesses, 59

in Bronze Age, I 1

Amasa, 323

Amelanchier alnifolia, $32 \mathrm{I}$

Ameloblasts, 94

Americans, Central, caries, I 2

North, caries, I2

South, caries, I2

Ammonia in saliva, 229

Anæmia, saliva in, 230

Anatomy of mastication, 36

Aneurism, saliva in, 230

Anglo-Saxon teeth, I I, 74

Antibiosis, forces of, in mouth, 285

Antiseptic properties of saliva, 228

dentifrices, 265

objections to, 269

Apotheosis of mechanical prophy.

laxis, 274

Apparatus, odontograph, 82

saliva segregator, 156

sclerometer, I25

Apteryx, 3I 2

Arabian dentistry, 3

Armenian teeth, 12

Arrested caries, 72

Arthritis, suppurative dental, 26

Artifact lines, enamel, 88

Artificial conditions, caries under, I 3

Asi-tree fruit, 3I 7

Asiatics, prehistoric, caries in, I 2

Attrition of teeth in native races, 330

Australian aborigines, diet, 32 f teeth, I2, 74

Axioms of bone development, 42

Bacillus fluorescens liquefaciens motilis, 20

furvus, 20

gingiva pyogenes, 20

maximus, $3^{\circ}$ 
Bacillus mesentericus, 30

fuscus, 20

ruber, 20

vulgatus, 20

necrodentalis, 20

subtilis, 20, 30

Bacteria, acid-forming, 20

alkali-producing, 286

dentine-liquefying, 20

Bacterial factors, reduction of, 265 plaques, 23

Bacteriology of immunity, 27, 30

Baden residents, caries in, Io

Banana, 3I7

Barracouta, 3I2

Beetle eaten by Papuans, 3 I9

Berries eaten by Indians, 321

Betel-chewing, 3I9

Beverages. See Drinks

Bilberry, 3I 5

Bile acids in saliva, 229 pigments in saliva, 229

Biliary secretion, tables, 298

Birmingham children, caries in, Io

Bitumen, 3I 3

"Black Club" methods, 274

Blastomycetes, 287

Bone development, axioms of, 42

Borolong kernels, 3 I 7

Bottle-feeding, $5^{8}$

Bread, aerated, 336

analysis of, 260

-fruit, 47

white, 259

whole-meal, 259

British Central Africa, diet, 322

Dental Association rules (I905), 7

race, caries in, $\mathbf{I} 3$

skulls, I I

Bronze Age, caries in, I I

Buccinator muscle, 56

Bulrush, pollen of, 3 I I root, 324

Burmese, caries among, I2

Bushmen, caries among, 9 diet, 323

Butter-fish, $3 \mathbf{I} 2$

Calcarine fissures, Io6

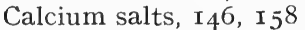

-magnesium balance, I 43

phosphates, I 36

Calcoglobulin, I 36

Calculus and oral sepsis, 28 r, 283

Cambridge Dental Clinic, I 2
Cannabis indica, 322

Cannibalism, 3 Io

Carbohydrate débris, 293

factor, 24I reduction of, 253,265

tables, 254, 255

Carbol-fuchsin, staining, I 8

Carbon dioxide, 25 content of saliva, 283

Caries, I 3

effect on enamel, 1 33,136

age incidence, I 5

and civilized diet, I 4

incidence of, in native races, 327

increase in, $\mathrm{xV}, \mathrm{I} I$

laboratory, 2 I

pathology of, I 8

results of, 357

sexual incidence, I 5

source of oral sepsis, 25

theories of, I 8

Carious teeth, culture from, $34^{\circ}$ comparison of, modern races, I2

Carnahuba palm, $32 \mathrm{I}$

Cassava, 321, 329

Central America, diet, $32 \mathrm{I}$ teeth, I 2

Chairs of dental research, $35^{6}$

Chemical equations, 20

theory of caries, I 8

Chemico-parasitic theory of caries, I9

Chewing, gum, 3I3

Chewing, habit of, $3 \mathrm{I}_{4}$

Children, caries in, Io

cleansing teeth, 269

correct diet, $34 \mathrm{I}$

hard food, 44

natural acids, 334

" tastes," 334

Chinese, caries among, I2, 74

dentistry, I

diet, 326

-white experiment, I9

Chinwans of Formosa, diet, 3 I 8

Chlorides in saliva, 2 I I

table, 2 I2

Cider, 335

Civilization, caries under, 13

diet of, and caries, I4

modern, dietaries of, 333

Climatic changes and caries, I 80

Cocaine on tongue, I77

Coccus, 30 
Cocoa-nuts, 3 I 7

Cod, 3I 2

Cold, common, 64 effects of, I 80

" Comforter," sucking, 54

Confectionery, 336 to be taxed, 36I

Congo red, 188

Cooking, Maori, 312 primitive races, 46

teachers of, 35I

Copper sulphate test, 201

Corynocarpus lavigata, $3 \mathrm{II}$

Cranberry, 3I 5

Crayfish, 3I 2

Crowding of teeth, 36 remedy for, 98

Crushing, in mastication, 44

Cucumber, $3 \mathbf{I} 7$

Cystitis, saliva in, 230

Cyttaria darwinii, $3 \mathbf{1} 6$

Decalcification of enamel, 248

Deciduous teeth, condition of, $5^{8}$ odontograms, 86

Density, determination of, rabbits' teeth, I 45

of enamel, 120

Dental arches, narrowing, 6I arthritis, suppurative, 26

Association, British, Io Schleswig-Holstein, Io caries. See Caries Clinic, Cambridge, I 2 detergents, ptyalin, I94 fruits, $34^{\circ}$

Dentifrice, urine as, 2 use of, 275

Dentifrices, alkaline, I 76 early, 6 effects on saliva, 176

Dentistry, preventive, 355

Dentists and prophylaxis, 354

Department of Public Health, suggested, 36I

Development of bone, axioms of, 42

Diabetes, oral sepsis in, 230 saliva in, $23^{\circ}$

Diagrams, importance of, $35^{\circ}$

Dialysis of saliva, 223

Diarrhœa, 343

Diet, Abyssinian, 323

Africa, natural races, 322

British Central, 322

Australian aborigines, 324

Bushmen, 323
Diet, Chinese, 326

Chinwans of Formosa, 318

civilized, and caries, I4

England, old times, 335

Esquimaux, 3I 5

Fuegians, 316

Cuachos, 329

India, races of, 325

Indians of North and Central America, 320

of North-West America, 316

Jakun, 320

Japanese, 326

Kabyles, 323

Kaffir, 323

Lapps, 3 I 5

Malay, 3 I 9

Manganga tribes, 323

Maori, 309

Melanesian, 3I6

Moors of Morocco, 324

Mota, 3I 8

native races, 328

negroes, West Africa, 323

New Hebrides, 3 I 8

Mexico, $32 \mathrm{I}$

non-stimulating, 302

North Asia, 3 I 5

Oceanian, 3 I 6

of immune races, 309

Papuan, 3 I 8

Persian, 326

Polynesian, 3 I 6

Pygmies of Central Africa, 323

Sakai, 320

Samoyedes, 316

Semang, 3 I9

softness of modern, 44, 333

Tasmanian aborigines, 324

tastelessness of modern. 333, 35 I

Todas, 326

Uganda, 322

Dietaries, acid, I 70

of 1,500 school-children, 337

of modern civilization, 333

Dietary recommended, 34I

Digastric muscle, 63

Diphtheroids, 30, 34

Diplococcus, 30

Disaccharides (equation), 20

Disposition of teeth and caries, 36,54

Dog, experiments on, I94, 300 teeth, odontogram, 87 
Driffield skulls, caries in, I I

Drinks, effect on saliva, I 70 recommended, 344

Dummy teats, 54

Durian fruit, 320

Early Iron Ages, caries in, I I

Ebers papyrus, I

Economic grounds for legislation, 359

Educational measures, $34^{8}$

Eels, 3 I 2

Egyptians, caries among, I2 dentistry, I tooth-paste, I

Electrical theory of caries, I 8

Emotional expression, 50

Enamel, abnormal, roI acids, effect of, 127

calcium magnesium balance, I 43

chemical composition of, I $4 \mathbf{I}$

$\mathrm{CO}_{2}$ solution, effect of, I33

decalcification of, 248

table, 249

density of, I20

hardness of, 44

histology of, 73

normal, Ior

permeability of, I35

physical properties of, 7I

rehardening, I37

solubility, comparative, I27

in. $\mathrm{HCl}, \mathrm{I}_{32}$

in lactic acid, I 28

in orange-juice, 130

striation in, 92

surface, civilized teeth, IO2

curvature of, 77

hardness of, I 24

imbrication lines, 74

in outline, 76

native teeth, ror

odontograph, 82

opaque spots, ro 8

origin of imbrication lines, 88

pathological fissures, I05 permeability of, II 2

physical properties of, I 12 staining of, I 6

thickness of, 44

Environment, 65, 94

Epithelial débris in saliva, 227

Equations, chemical, 20, 287, 292 density, I2I
Esquimaux, caries among, 9, I 2 diet, 3 I 5

Ethylidene lactic acid, 242

Eugenia, 320

Europeans, caries among, I2

Experimental evidence from lower animals, r8I, I94

Experiments, Chinese white, 9I neutralized foods, 302

Saccharomyces coagulatus, 29 I salivary glands, I 8I sapid substances, 28 I sulphocyanate, I99

Expression, emotional, 52

Extirpation of salivary glands, I94

Facial muscles, play of, 52

Fauces, muscles of, 55

Feeding-bottles, $5^{8}$

Fermentability of various sugars, 244

Ferments and their actions, 282

Fern-root, 3 ro

Ferric chloride test, 201

Fiji Islanders, teeth, 9, 74

Fikaks, 324 diet, 3 I 6

Finger-sucking, 54

Fish, Maori, 3I2

Fissures, calcarine, Io6 deep, ro7 sinuous, ro7

Foods, softness of modern, 44, 333 staple, 47

Foodstuffs, lodgeability of, 249 acidity or alkalinity of, 252

Forks, use of, 42

Formalin, 267

Formulæ for mouth-washes, 278

Fruit for children, $34^{\circ}$ native races, 3 I I et seq.

Fruits, tinned, 343

Fuchsia excorticata, 3 II

Fuegians, 3 I 6

"Fur" upon tongue, 303

Gastric secretion, 296 tables, 296, 300

Gastro-enteritis, saliva in, 230

Genio-hyoglossi muscles, 39 hyoid muscles, 39, 63

Germany, North, caries in, Io

Ginger, wild, 320

Gingivitis, 27

Glands, salivary, experiments on, I 8I 
Glands, salivary, extirpation of, 194

Glass-house simile, 362

Glosso-pharyngeal reflex, 300 pharyngeus muscle; $5 \mathrm{I}$

Glucose in lactic-acid fermentàtion, 20

in saliva, 229, 289

Glycogen in saliva, 229

Gnatho-dynamometer, 45

Gothic palates, 60

Grape cure, 345

Graphite staining, 73

Graves's disease, saliva in, 230

Greek dentistry, I

Gustatory stimuli, elimination, I 82

Habits, sucking, 54

Halifax children, caries in, ro

Hambourg residents, caries in, ro

Hapuka, 3I 2

Hard foodstuffs, 46

Hardness of water, $\mathbf{I}_{4} 6$

Hawaii, food in, 48

Healed hypoplasia, I I

Heredity, 36, 65

Hinau-tree, berries, 3I I

Hindoos, caries among, I2

diet, 325

Northern, 9

Southern, 9

teeth, 74

Histological examination, enamel, 73

Honey, 336

Honeycomb, 320

Hors d'ceuvre, 34I

Hué, 3II

Hydrochloric acid, solubility of enamel in, 132

Hydrogen dioxide, 267 peroxide, 267

Hylobates, 97

Hyphomycetes, 286

Hypoplastic teeth, I Io

Imbrication lines, 74,90 hypothesis, 94

Immune bodies in saliva, 225

Maori children, 28

mouths, bacteriology of, 27

races, diet of, 309

Immunity and mouth organisms, 294

flora of, 35

of native races, 294
Inangi, 3I 2

Incision (in mastication), 43

Incremental lines, I ro

Index, alkalinity, etc., I 58

India, races of, diet, 325

Indians, North American, 9 diet, 320

North-West American coast, 9 diet, 3I 7

Indol, 25

others, 3I8, 32 I

Inoculation of the mouth with other organisms, 285

Insalivation, effect of, 244

tables, 245

Interprismatic spaces, I I 8

Ipomiea chrysorrhiza, 3 Io

Jakun, diet, 320

Japanese, caries among, I 2

diet, 326

Java, aborigines of, 9

Jaws, and caries, 36

Kabab, 324

Kabyles, diet, 323

Kaffir diet, 323

Kaka, 3I2

Karaka, 3I I

Karengo, 3I I

Kauri gum, 254, 3I3

Kava, 318

Kesk'soo, 324

Kettering children, caries in, ro

King-fish, 312

Kiwi, 312

Knives, use of, 42

Kor, 47

Koreké, 312

Kukupa, 3I 2

Kulim leaves, 320

Kumara, 48, 3 Io

Kutukutu, 3I I

Laboratory caries, 2 I

Lactic acid in saliva, 229 solution of enamel in, $\mathbf{I} 28$

fermentation, 20 in vitro, 242

Lampreys, 3I 2 tables, 243

Lapps, diet of, 3I 5

Leavening, old-fashioned, 336

Lectures on oral prophylaxis, $35^{\circ}$

Legislation, form of, 360 need for special, 357 
Leith children, caries in, ro

Lemonade, home-made, 344

Lemons, sucking, I64

Leptothrix innominata, $3 \mathrm{I}$ racemosa, 30

Leucine in saliva, 229

Levator palati muscle, $5 \mathrm{I}$

Lilium columbianum, $32 \mathrm{I}$

Lime-juice, 344

Lingualis superior, $4^{\circ}$ transverse, $4^{\circ}$

vertical, $4^{\circ}$

Lip-sucking, 54

Liquids, effects of, upon saliva, I 70

Litmus as indicator, $\mathrm{I} 88,24 \mathrm{I}$

Locomotor ataxia, saliva in, 230

Lodgeability of foodstuffs, 249 table, 252

Loko, 47,318

Lot, 47,318

Luxuries, taxation of, 36I

Lysol, 267

Madagascar, aborigines of, 9

Madeira, diving-boys of, I8o

Magnesium salts, I 46, I 58

Maize, 47,48 , 32I, etc.

Malacotic teeth, 72 , I 02 odontogram, 84

Malay diet, 319 Peninsula, aborigines of, 9 prehistoric, caries among, I 2

Maltose, 2I, 229

Manganga tribes, diet, 323

Mango, 3I 7

Manioc, $32 \mathrm{I}$

Maori birds, 3I I caries among, 9 children, I2, I 3,28

civilized, I2

cooking, 48, 3I 2

enamel, 79

fish, 3I 2

food of, 48, 309

gum-chewing, $3 \mathrm{I} 3$

immunity, 28, 3I 5

odontograms, 85

race, 309

teeth, 9, I3, 74, I I 5, I 29, I 3 I uncivilized, 12

Mariba palm, 32I

Masseter muscle, 37

Mastication, anatomy of, 36 crushing, 44 incision, 43
Mastication, physiology of, 36 prehension, 42 table, 53 triturition, 45

Masticatories, 3I3, 3I 6

Meat, consumption of, 48

Medical practitioners and prophylaxis, 352

Melanesian diet, 3 I 6

Mercuric chloride, 267

Messalina, dentifrice of, 2

Methyl orange, I 88

Mett's tubes, method, 190, 208

Mexican teeth, 9, 74

New, diet, $32 \mathrm{I}$

Micrococcus catarrhalis, 30

Millet, 47, 322, 325, etc.

Moki, 3I 2

Monosaccharides (equation), 20

Moors of Morocco, diet, 324

Moriori teeth, 74

Mota diet, 3 I 8

Mouth-breathing, 62, 64

Mouth-washes, antiseptic value of, 266,275 acid, formulæ for, 278

Mucin, 158,2 I 3 table, $2 \mathrm{I} 6$

Mucinometer, 217

Muscles of mastication, 37 genio-hyoglossus, 39 genio-hyoid, 39 internal pterygoid, 38 masseter, 37 mylo-hyoid, 38, 63 temporal, $4^{\circ}$ of tongue, $4^{\circ}$

Mutton birds, 3I2

Mycology of the mouth, 28

Mylo-hyoid muscles, 38, 63

Nasal cavities, 6I

Nasmyth's membrane, I35, I37

Native teeth, 72, Ior, 330

Natural conditions, caries under, I 3

Need for special legislation, 357

Negro influence, 66 slaves, caries in, 9

teeth, 74

West Africa, diet, 323

Neolithic skulls, io

Nephritis, chronic, 27 saliva in, 230

Nerve, vagus, 30 I

Nerves, cranial, I 86 
Nerves of taste, I 5 I, 298

sympathetic, I 52, I 86, 304

Neurasthenia, saliva in, 230

Neutralized food, experiments, 320

New Caledonia, aborigines of, 9

Hebrides, diet, $3 \mathbf{1} 8$ teeth, 74

Guinea, teeth, 74

Mexican diet, $32 \mathrm{I}$

Nicotine, I 77

Nipple, artificial, 55

Oceanian diet, $3 \mathrm{I} 6$

Odontograms, 84

Odontograph, 82

Opaque spots, enamel, ros

Opsonins, 225

Oral hygiene, lessons, 349

sepsis and calculus, 283 from dental caries, 25 saliva in, 230 sources of, 26

Orange-juice, solubility of enamel in, 130

Orbicularis oris muscle, $44,5^{\circ}$

Orphanage children, 66

Osteitis, rarefying, 27

Oxygenetic factors, 24I, 265

Pain, effect of, on saliva, 223

Paiwari, 3 I 8

Palates, shape of, 60

Palato-glossus muscle, 56 -pharyngeus muscle, 56

Paleolithic skulls, Io

Palm, carnahuba, 32 I mariba, $32 \mathrm{I}$

Pancreatic reflex, 301 secretion, 297

table, 297

Papuan, edible beetle, 319 diet, 318

Paradise duck, 3 I 2

Parotid secretion, 182,185

Parrot, 3I 2

Patagonian teeth, 9

Pathology of dental caries, i 8

Patiki, 3I 2

Paua, 3I2

Pepper, wild, 320

Persian diet, 326

Peruvian teeth, 9

Phagocytosis in saliva, 32, 227

Pharynx, constrictors of, 56

Phenolphthaleĩn, I 88
Phosphates in saliva, I $36,158,210$ table, 2 I I

Phthisis, saliva in, $23^{\circ}$

Physical deterioration report, Io, 359

Physiology of mastication, 36, 4 I

Pig, Fijian, 3 I 7

Mota, 3 I 8

Pigeon, 3 I 2

Piharau, 3 I 2

Pilocarpine nitrate, $\mathbf{r} 86$

Piper methysticum, 3 I 8

Plaques, bacterial, 23

Pneumococcus, 30, 34

Poi, 3 I 7

Polarized light, examination by, 9 I

Polynesians, caries among, I2 diet of, 3 I 6

Eastern, caries among, 9

Polysaccharides, 2 I

Poporo, 3I I

Poroporo, 3I I

Post-nasal growths, 49

Potassium tartrate, as dentifrice. $28 \mathrm{I}$

Potential, acid, diet, 263 alkaline, 264

Prehension, 42

Prehistoric crania, caries in, I 2

Preventive dentistry, 355

Primitive races, existing, caries in, 9

cooking, 46

soft food of, 49

Prisms, enamel, 93, I I

Prophylaxis and diet, $34^{\mathrm{I}}$ in textbooks, 355 mechanical, 274

Protection, natural, forces of, 69

Protein and carbohydrate mixture, 247

Psychical secretion, I52

Pteris aquilina, 310 esculenta, 3 IO

Pterygo-maxillary ligament, $5^{6}$

Pterygoid muscles, internal, 38

Ptyalin, absence of, 229 digestion of starch by, 197 estimation of, I90 table, 192

Public Health Service, 36I

Pueko, 312

Putangitangi, 3I 2

Pycnometer, I 45

Pygmies, Central Africa, rliet, 323 
Pyorrhœa alveolaris, 26

Pyrethrum, 221

table, 222

Quail, 312

Quinoa, 32 I

Rabbits, experiments on, I44, I 8I, 194, 302

Races, primitive, caries in, 9

Raratongan teeth, 74

Rarauhe, 310

Reflex arc, 298

blocking of, $30 \mathrm{I}$

digestive secretions, 298

pancreatic secretion, zor

Research work, 356

Resistance of enamel, I 24

Retzius, striæ of, 88 , I I 0

Rhinitis, posterior, 49

Rice, $47,325,326$

Riwai, 48

Romano-British skulls, caries in, I I

Saccharomyces albicans, 288

coagulatus, 289

experiments, 29r

Sago, $3 \mathbf{I} 7$

Sakai diet, 320

Salads, 34I

Saliva, I 49

abnormal constituents, 229

acid dietary, effects of, I 70

acids, effects of, I 69

alkalinity of, I 59 et seq.

amount of, I 59 et seq.

antiseptic properties of, 228

apparatus for obtaining, 156

chlorides, 2 I I

collection of, I 55

composition of, I 57

conclusions, 230

conditions of normal secretion, 150

cranial nerve stimulation, I 86

dentifrices, effects of, 176

depressants of, I 75

dialysis of, 223

diminished taste perception. I 77

effect of pain on, 222

thyroid extraction, 235

epithelial débris in, 227

experiments, I $8 \mathrm{I}$

. How of, $15^{8}$
Saliva, immune bodies in, 225

in disease, 230

lemon-sucking, effects of, I 64

liquids, effect of, I 70

methods of obtaining, I 53.

I 55

mucin in, $2 \mathrm{I} 3$

neutralizing power of, 187

phagocytosis in, 32, 226

phosphates, I36, 2 I0

psychical secretion, I 52 effects, 178

ptyalin, 189

reaction indicators, 187

reflex arc, $\mathbf{I}_{52}$

resting, I60

rinsing effect, 154

ropy, 2 I 5

secretion, normal, I 50

psychical, I 52

under pain, 223

variations, 160,165

stimulation of, I $55 \mathrm{et} \mathrm{seq,22} \mathrm{I}$

sulphocyanates in, 198

sympathetic stimulation, I 86 , 304

tables, I6I, I66, I69, I 7 I, I 72,176, I 79

titration of, I 59

viscosity of, 2 I 8

Salivary depressants, I62, I67, I $75,178,253$

excitants, 253

glands, extirpation of, 194

stimulants, I 59 et seq., 22 I

Samoyedes, diet of, 3 I 6

Sandwich Islanders, caries in, 9

Saori kernels, 3I 7

Sapid substances, experiments, 281

Sapidity in diet, need for, 328

Sapræmia, 27

Sarcina, $5^{\circ}$

alba, 20

aurantica, $2 \mathrm{O}$

Iutea, 20

Sauces, acid, 342

School-children, caries in, Io dietaries, 337

Schreger's lines, 77,88

Sclerometer, I25, I 45

Sclerotic teeth, 72, IO3 enamel in, I05

odontogram, 86

Segregator for saliva, ${ }_{5} 6$

Semang tribes, diet, 3I9 
Sepsis v. suppuration, 25

Septic theory of caries, 19

Septicæmia, 357

Sexual incidence, caries, I5 selection, 67

Sham meals, 300

Shark, 3I2

Shellfish, 3I2

Sicilian teeth, 164

Silver nitrate stain, I I 2

Sinuous fissures, 107

Skatol, 25

Skiagrams, 56, 100

Snake-charmers, immunity of, 225 saliva of, 225

Snapper, 3I 2

Sodium salts, $127, \mathbf{1} 5^{8,}$ 167

Solanum aviculove, 3I I nigrum, 3 I I

Solubility of enamel, 127 qualitative, I 28, I 30 quantitative, I 32

Soured milk treatment, $34^{6}$

Speech, effect of, $5^{\circ}$

Spivilla, 30

Spirocheta dentium, 30

Staphylococcus, 30

albus, 20, 34

aureus, 20, 34

cerevisice, $29 \mathrm{I}$

viscosus, 290

Staple foods, Fiji, 47

Mota, 47

Starch, digestion by ptyalin, 197

in rabbits' fæces, 196

Starches and sugars not to be eaten alone, 264

Steaming food, 312

Stellate reticulum, I 35

Stenson's duct, cannula for, ${ }_{5} 6$

Stomatology for students, 354

Stone Age, caries in, II

Strawberries, Chile, 32 I

Streptococcus bacillus, 34

brevis, 20, 30, 34

longus, 30, 34

maximus, $3 \mathbf{I}$

Stylo-glossus, 5I

Sublingual glands, segregator for, I 56

Submaxillary gland secretion, I 85 segregator for, ${ }^{5} 5^{6}$

Suckling, natural, 58

'Sugar-cane, 257, 3 I 7

consumption of, 336

danger of, 217,344
Sugar-pine, wood of, 316

Sugars, fermentability of various, 244

Sulphocyanate experiments, 199 internal administration, 203 origin of, 205

Sulphocyanates, 158,198 table, 202

Sulphuretted hydrogen, 25

Suppuration $v$. sepsis, 25

Suppurative dental arthritis, 26

Susceptibility of different teeth, I6

Swallowing, 49

Swamp-hen, 312

Sweetmeats, 336, 344

to be taxed, 361

Sweet potato, 3 Io

Syphilis, 27 saliva in, 230

Tables, analysis breads, 260 antiseptic mouth-washes, 268 bacteria, 20, 30, 34

biliary secretion, 298

carbohydrate factor, 254, 255

chlorides in saliva, 212

composition of enamel, I42, I 45

decalcification of enamel, 249 density of enamel, 122, 124

dietaries, school-children, 337 gastric secretion, 296, 300 glands, weight of, 183 . hardness of enamel, I 26 incidence of caries, 9, Io, I I,

I 2, 17

insalivation, effect of, 245

lactic fermentation, 243,248 , 263

lodgeability of foodstuffs, 252 masticatory muscles, 53

mouth - washes, approved, 278,279

mucin, 216

muscles of mastication, 53

neutralization of acids by saliva, r 88

pancreatic secretion, 297

permeability of enamel, II3,

I 15

phosphates in saliva, 2 I I, 224

ptyalin, I92

pyrethrum, 222

roller-milling, effect of, 260

saliva, I6I-I 79 
Tables, saliva after pain, 223

sham feeding, 300

solubility of enamel, I 29, I32

starch in fæces, 196

converted into maltose, 197

sulphocyanates, 198, I99, $202,203,205.206,207,208$

thyroidectomy, 233, 235, 237

viscosity of saliva, 2 r 9

yeast, 292

Taboo, 329. See Tapu

Tahiti, food in, $4^{8}$

Tapu (food), 3I o, 3I I

Tarata gum, 313

Taro, 47, 3II, 3I 7

Tasmanian aborigines, diet, 324

Taste perception, diminished, 177 ,

Tartar, 5

Taxation of luxuries, 361

Tea-drinking, $14: 8,170,335,342$, 344

Teachers in primary schools, 348 et seq.

Teat, dummy, 54 natural shaped, 57

Temporal muscle, 37

Tensor palati muscle, 5 I

Theories of dental caries, I 8 chemical, i 8 chemico-parasitic, I9 electrical, 18 humoral, i 8 septic, I9 worm, 18

Thirst, relation to caries, 167

Thyroglossal duct, 235

Thyroid deficiency, 232

Thyroidectomy, 233

alimentary absorption after, 233

calcium amount after, 233

composition of the teeth after, 234

effect on salivary secretion, 235

table, 237

Ti-tree root, $3 \mathbf{I} \mathbf{1}, 3 \mathbf{I} 7$

Tobacco-smoke, 284

Todlas, diet of, 326

Tome's processes, Io4
Tongue, action of sapid substances 281

fur upon, 303

muscles of, $4^{\circ}$

Tooth-brush, form of, 272

manner of using, 270,273

-paste, Egyptian, I

-picks, Roman, 2

Saxon, 2

-powders ancient, I, 2, 4

-stick, 270

Totemism, 329

Tough foodstuffs, 46

Toxæmia, chronic, 27

Trituration in mastication, 45

Tsang-eul-tsee, I

Turmeric, 320

Tutin, $3{ }^{1} 4$

Tutu-berry juice, 3II, 3I 4

Typhoid, saliva in, 230

Uganda, diet, 322

Upokororo, 31 2

Urea in saliva, 229

Urine as dentifrice, 2

Uriwera reserve, 28

Variety in diet, need for, 328

Vegetarian diet, 34I

Vibrios, comma-shaped, 30

Vicious circle, alimentary, 299 nasal, 63

Viscosity of saliva, 218

Vision, effect of, 52

Vitamines, 262

Wainama, 313

Water, drinking, importance of, I 43

Weka, 312

Wheat, tables, 260

White bread $v$. whole-meal, 259 flour, fine, to be taxed, $36 \mathrm{I}$

Whole-meal bread, effects, 263

Wine, drunk in England, 335

Wood-hen, 3I 2

Worm theory of caries, 18

Yams, $47,317,318$

Yeast, 287, 29I

Zulus, caries among, 9 


\section{AUTHORITIES}

(Bibliographical references in thick type).

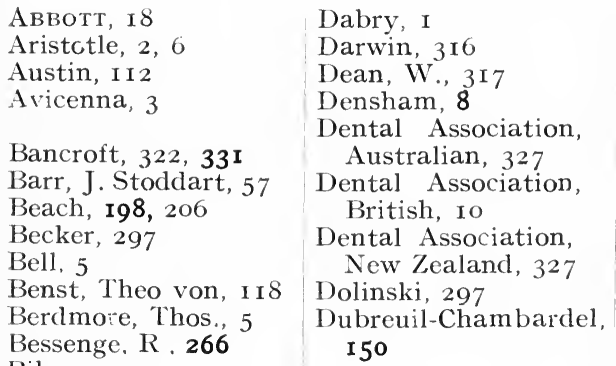

Bibra, von, I 4 I

Bidder, 183

Black, 23, 44, 45, 72, $73,8 \mathrm{r}, 345$

Blagden, $33^{2}$

Borisov, I9I

Bridgeman, I 8

British Dental Association, Io

Brown, J. M., 33 I

Bunge, I 43, 344

Bunon, 4

Bunting, 20 I

Cannon, I 89, I 97

Carlson, r86, 230

Celsus, 2

Champtaloup, 28

Chassevant, 269

Chittenden, I97

Chouppe, 198

Collier, Mayo, 62

Colyer, 60, r6o

Cook, 2 I 4

Cook, Captain, $25^{8}$

Cooper, 302

Crittenden, I86, I87

Crooke, 331

Cunningham, IO, I2, I 5, I 7
Dabry, I

Densham, 8

Dental Association, ental Association,

New Zealand, 327

Dolinski, 297 I5O

Ebers papyrus, I

Edkins, 296

Ellenberger, 198, 246

Ellis, 258

Fauchard, Pierre, 4

Favre, 320

Fehr, I94

Fenwick, 205

Fitchett, F., 3 I 4

Fleckseder, 230

Fleig, 30I

Florian, 198

Floxas, 267

Forest, of Leyden, 3

Fox, 5, 8, I 8

Frerichs, 198

Gabell, I 12

Gamgee, 229

Gassmann, I4I

Geyger, of Leipsig, 198

Gillen, 332

Giovanni, of Arcoli, 3

Goadby, 20, 28, 229, $286,288,345$

Goddard, 5, 6, 8

Greve, I I, I6, I7

Greyger, 203
Guerini. 8

Guppy, 317, 33I

Haddon, 33 I

Haidenhain, I74, I83

Hakluyt, 42

Halifax, M. O. H., Io

Harden, 289

Harris, 5, 8, I 8

Haycraft, J. B., 28I

Head, 127, I33, I37, I 88

Henderson, 8

Hill, Leonard, 262

Hill-Tout, 33I

Hippocrates, 2, I 8

Hitchcock, I7

Hofmeister, 198, 246

Hoppe-Seyler, I I 7, I 4 I

Howitt, A. W., 33 I

Hugenschmidt, 198

Hunt, 267, 269, 270, 280

Hunter, 5, I 8

Hutchison, 244, 260, 344

Jacubiowitsch, I98

Johnson, 2 I4

Junker, of Halle, 4

Kenwood, I 46

Kirk, 73, 8I, I99, 2 I 4

Kjellmann, 3 I 5

Kölliker, 90

Külz, I 33

Langley, I55, I83, I84, I 86

Layton, Io, $\mathbf{1 7}$

Lewis, J. M., of Melbourne, I33, I43

Lister, 6 
Lloyd, A. B., 33I

Lobasov, 296, 298, 299

Logan, 320

Low, I98, 206

Mackenzie, 60

Magitot, 9, I5, I7

Majendie, 262

Malcolm, I44

Marshall, Professor, $4^{8}$

Matthews, 29I

Mayer, A., 289

Mayer-Tidy, 204

Meakin, 33I

Michaels, 229

Michel, of Wurzburg, I98, 206

Miller, 6, I9, 28, 7I, $8 \mathrm{I}$, I 33, I50, I99, $219,225,228,268$, $269,284,288,33$ I

Milles, 19

Minkowski, I94

Mitscherlich, I98

Moore, 194, 198

Morris, G. H., 289

Mosler, 230

Mummery, J. R., 9, I I, I7, 309, 320, 327

Nunk, 198

New Zealand Institute, 332

Nuck, Anton, 4

Oehl, I98

Oppenheim, 199

Oppenheimer, 282, 288, 289

Osler, 359

Ottofy, I2, I7
Parkes, 146

Pasteur, 6

Patrick, I2, I7, 327, 329

Paul, of. Agina, 2

Pavlov, I50, I5I, I62, I68, I78, I9I, I97, 296, 298, 299, 30 I

Pepys, 335

Pliny, 2

Pratt, 331

Ratzel, 320, 328, 33 I

Read, T. G., 263

Richards, IO, I 5 , I7

Richet, 269

Rigolet, 150

Rivers, 332

Rivière, 4

Robertson, IO, I7, 244

Romberg, 26I

Röse, of Munich, 7, Io, I 5,17, I 43, I 46

Rubner, 26I

Ryan, 230

Sanarelli, 229

Schäfer, I33, I 55, I83, I84, I94, I98, 229, 230, 28 I

Schmidt, 198

Schütz, I9I

Seligmann, $33^{2}$

Sellheim, I53, I54, I86, Williams, Leon, 23, 2 I 8

Simpson, Sir George, 48

Skeat, 320, 332

Smale, I6o

Smith, D. D., Philadelphia, 274
Smith, Hopewell, is, $8 \mathrm{I}$

Snyder, 26I

Soemering, 204

Spencer, 332

Stark, 262

Starling, I5I, 296

Talbot, 6I

Tassinari, $28_{4}$

Taylor, 332

Thomas, N. W., $33^{2}$

Tomes, 19, 7I, 72, 81, I 4 I

Tregear, $33^{2}$

Underwood, I9

Vintschgau, M. v., $28 \mathrm{I}$

Wadsworth, 266

Walkoff, 9o

Waller, I 33, 232

Wallis, $34^{8}$

Waugh, 207

Weiske, 144

Werner, A., 332

Wertheimer, 3OI

Westcott, 5, I 8

Westrumb, 204

William of Ruysbruck, 42

$7 \mathrm{I}, 73,81,90,232$, 345

Wright, 2, I I, I7

Young, 289

Ziem, 62
Wallace, 333 


\section{$\mathrm{ON}$ DENTISTRY}

SEWILL'S DENTAL SURGERY, including Special Anatomy and Pathology. A Manual for Students and Practitioners. Fourth Edition. Edited by W. J. ENGLAND, L.D.S. Eng.; and J. Sefton Sewill, L.R.C.P., M.R.C.S., L.D.S. Eng. Pp. xii +622 , with 28I illustrations. Price ros. 6d. net (postage 5d.).

TIGHTENING OF LOOSE TEETH : Some Technical innovations. By Surgeon-Dentist Witkowski, of Berlin. Translated by Edgar Neumann, M.D., and William M. Gabriel, M.R.C.S., L.D.S. Eng.

Pp. xii +74 , with 9 plates and 33 figures in the text. Price 4 s. net (postage $3 \mathrm{~d}$.).

PORCELAIN FILLING OF TEETH (INLAYS.) A Guide to the Filling of Teeth with Porcelain and its application in some special cases. By H. J. Mамцоск, Dental Surgeon, Berlin. Translated from the Second German Edition by WM. M. GabRiel, M.R.C.S., L.D.S.

Pp. $x i i+176$, with 173 illustrations. Price 4 s. net (postage $3 d$.).

NOTES ON DENTAL METALlURGY. By W. Bruce Hepburn, L.D.S. Glasgow, Lecturer on Dental Metallurgy, Glasgow Dental Hospital and School, etc.

Pp. viii + 2r 4 . Price $5 \mathrm{~s}$. net (postage $4 \mathrm{~d}$ ).

STUDIES IN COMPARATIVE ODONTOLOGY. By ARTHUR S. Underwood, M.R C.S., L.D S. Eng., I'rofessor of Dental Surgery, King's College, London.

Pp. viii $+{ }_{5} 2$, with 77 original illustrations. Price 5 s. net (pos iage 4 d.).

PRACTICAL CHEMISTRY, including Simple Volumetric Analysis and Toxicology. By P. A. Ellis Richards, F.I.C., F.C.S., Lecturer on Chemistry and Metallurgy at the Royal Dental Hospital. etc. Pp. $x+$ i 5o. Price 3s. net (postage 3d.).

BOOK-KEEPING FOR DENTISTS. By Frank White. Specially designed for the busy dentist who desires to keep his accounts in the most practical manner possible.

Pp. iv +60 . Price rs. 6d. (postage 2d.).

AIDS TO DENTAL ANATOMY. By Arthur S. Underwood, M.B., M.R.C.S., L D.S Fng.

Second Edition. Price, cloth, 2s. 6d, net ; paper 2s. net (postage 2d.)

AIDS TO DENTAL SURGery. By Arthur S. Underwood, L.D.S. Eng., and Douglas GabelL, M.R.C.S., L.R.C.P. I.ond, L.D.S. Eng.

Third Edition. Price, cloth, 2s. 6d. net; paper 2s. net (postage 2d.). 


\section{SELECTED PUBLICATIONS}

FROM THE CATALOGUE OF

\section{BAILLIĖRE, TINDALL \& COX}

MINOR MALADIES AND THEIR TREATMENT, including Colds, Coughs, and Sore Throats-Indigestion-Constipation, Diarrhœa-Rheumatism, Neuralgia, Headache-Goutiness-General Health-Some Drugs, and their Uses. By Leonard Williams, M.D., Physician to the Metropolitan Hospital.

Third Edition. Pp. viii +396 . Price 5s. net (postage 4 d.).

FOODS: THEIR ORIGIN, COMPOSITION, AND MANUFACTURE. By William Tibbles, M.D., Medical Officer of Health, Melton Mowbray. Pp. viii +95o. Price r8s. net (postage 6d.). "It is the most complete work on foods and beverages published." -Chemist and Druggist.

THE HAIR AND ITS DISEASES, including Ringworm, Greyness, and Baldness. By David Walsh, M.D., Senior Physician, Western Skin Hospital, London. Second Edition. Pp. vi+96, with 6 illustrations. Price 2s. 6d. net (postage 3d.).

PREVENTION OF COMMON DISEASES IN CHILDHOOD. By J. Sim Wallace, M.D., Dental Surgeon, London Hospital. Pp viii + Io4. Price 3s. 6d. net (postage 3d.).

" The ideas s.t forth in this book are worthy of close attention, for there is no doubt that an immense amount of disease and pain could be obviated."-Sheffield Daily Telegraph.

MEDICAL SUPERVISION IN SCHOOLS : Being an account of the systems at work in Great Britain, Switzerland, Germany, Canada, and the United States. By E. Millar Steven, M.B., Royal Commissioner for South Australia.

Pp. $x+268$, with 40 illustrations. Price 5s. net (postage 5d.).

\section{NURSERY NOTES FOR MOTHERS. By C. WilletT} Cunnington, M.B., late Clinical Assistant, Hospital for Sick Children, Great Ormond Street, and the Evelina Hospital for Sick Children. Pp. vi + I44. Price 2s. 6d. net (postage 3d.).

MANUAL OF NURSERY HYGIENE. By W. M. Feldman, M.B., Lecturer and Examiner in Hygiene, Infant Care, Nursing, etc., to the London County Council.

Second Edition. Pp. xiv + I68, with 3 plates and 45 illustrations. Price, cloth 2s. 6d. net; paper, rs. 6d. net (postage 3d.).

HOME NURSING. By B. Myers, M.D., late Lecturer and Surgeon to the St. John Ambulance Association.

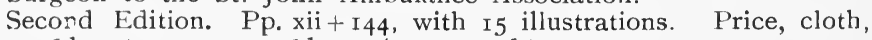
2s. $6 \mathrm{~d}$. net ; paper, Is. $6 \mathrm{~d}$. net (postage $3 \mathrm{~d}$.). 


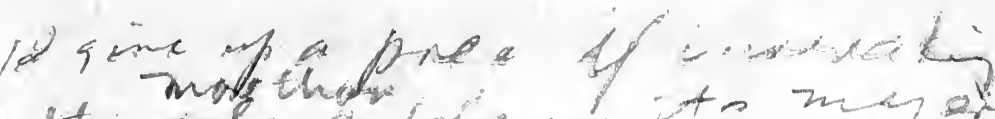

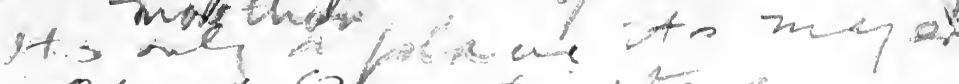

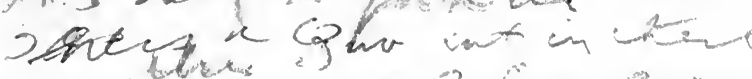

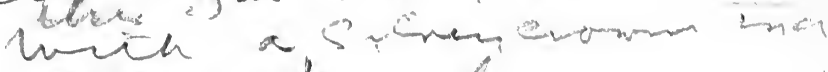

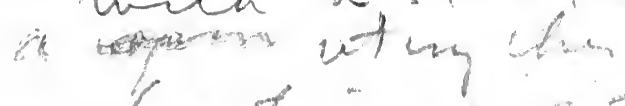
2 a reatginats

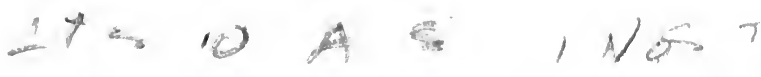

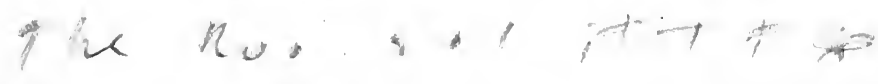

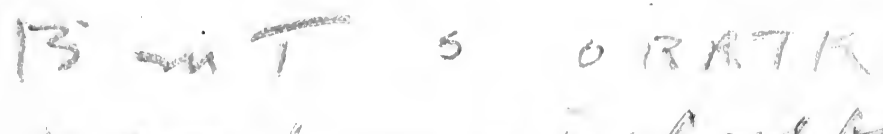

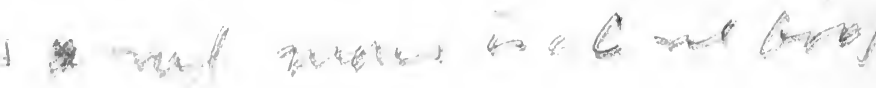




\section{DAY USE}

RETURN TO DESK FROM WHICH BORROWED

This book is date of the last date stamped below, or on the date to which renewed.

Renewed books are subject to immediate recall.

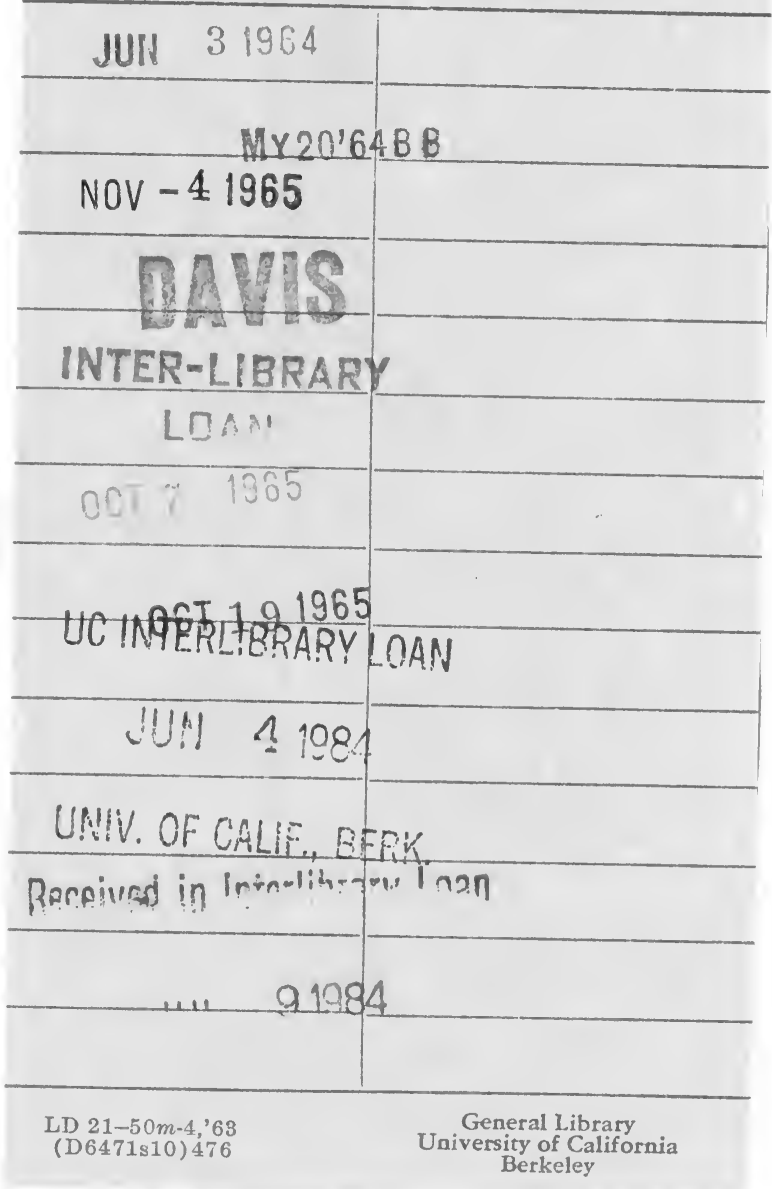


450

갈 
